\title{
Eupolars and their Bialternality Grid
}

\author{
Jean Ecalle ${ }^{1}$
}

Received: 2 June 2014 / Revised: 13 January 2015 / Accepted: 14 January 2015 /

Published online: 7 October 2015

(C) The Author(s) 2015. This article is published with open access at Springerlink.com

\begin{abstract}
This monograph is almost entirely devoted to the flexion structure generated by a flexion unit $\mathfrak{E}$ or the conjugate unit $\mathfrak{O}$, with special emphasis on the polar specialization of the units ("eupolar structure"). (i) We first state and prove the main facts (some of them new) about the central pairs of bisymmetrals $\mathrm{pal}^{\bullet} / \mathrm{pil}^{\bullet}$ and $\mathrm{par}^{\bullet} / \mathrm{pir}^{\bullet}$ and their even/odd factors, by relating these to four remarkable series of alternals $\left\{\mathfrak{r} \mathfrak{e}_{r}^{\bullet}\right\},\left\{\mathfrak{l} \mathfrak{e}_{r}^{\bullet}\right\},\left\{\mathfrak{h} \mathfrak{e}_{r}^{\bullet}\right\},\left\{\mathfrak{k} \mathfrak{e}_{2 r}^{\bullet}\right\}$, and that too in a way that treats the swappees pal ${ }^{\bullet}$ and pil ${ }^{\bullet}$ (resp. par ${ }^{\bullet}$ and pir $^{\bullet}$ ) as they should be treated, i.e., on a strictly equal footing. (ii) Next, we derive from the central bisymmetrals two series of bialternals, distinct yet partially (and rather mysteriously) related. (iii) Then, as a first step towards a complete description of the eupolar structure, we introduce the notion of bialternality grid and present some facts and conjectures suggested by our (still ongoing) computations. (iv) Lastly, two complementary sections have been added, to show which features of the eupolar structure survive, change form, or altogether disappear when one moves on to the next two cases in order of importance: eutrigonometric and polynomial.
\end{abstract}

Keywords Multiple Zeta values · Flexion structure

Mathematics Subject Classification (2010) $11 \mathrm{M} 32$

Jean Ecalle

jean.ecalle@math.u-psud.fr

1 Chercheur au CNRS (D.R.), Equipe d'Analyse Harmonique, Mathmatiques, Bt. 425, Université Paris-Sud, 91405 Orsay Cedex, France 


\section{Contents}

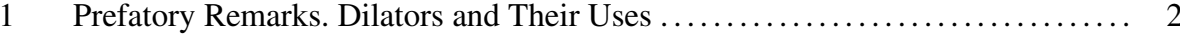

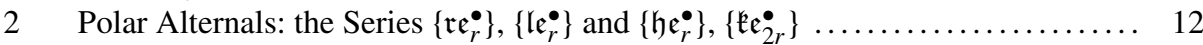

3 Polar Bisymmetrals: Main Statements .............................. 19

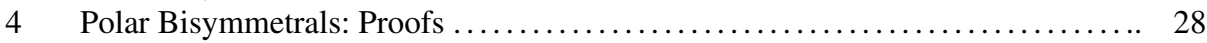

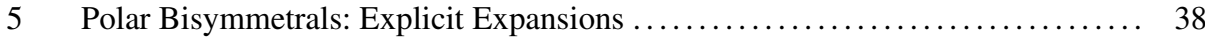

6 Polar Bisymmetrals: Seven Remarks .................................. 42

$7 \quad$ Essential Parity of Bialternals ...................................... 45

8 Standard Factorization of Bisymmetrals ............................. 47

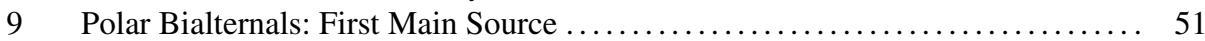

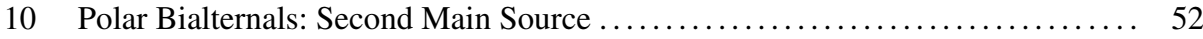

11 Polar Algebra and Subalgebras .................................... 55

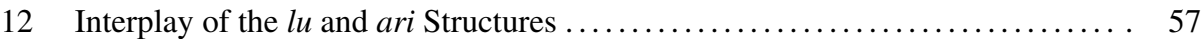

13 Alternal Codegrees and Alternality Grids ........................... 58

14 Bialternal Codegrees and Bialternality Grids $\ldots \ldots \ldots \ldots \ldots \ldots \ldots \ldots \ldots \ldots \ldots \ldots$

15 Introduction to the Polynomial Chessboard ......................... 63

16 From Polar to Trigonometric Bisymmetrals .......................... 76

17 Basic Prerequisites ........................................... 80

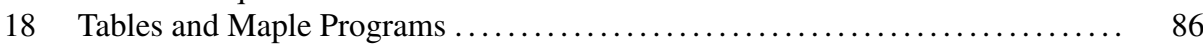

\section{Prefatory Remarks. Dilators and Their Uses}

\subsection{Preamble}

We assume some familiarity with [2] or [4], though the main definitions have been recalled towards the end, in the appendix Section 17. In the main, the present paper concerns itself with the simplest, most basic flexion structure, namely the multialgebra-cum-multigroup Flex $(\mathfrak{E})$ generated by a single flexion unit $\mathfrak{E}$, and the companion structure Flex $(\mathfrak{O})$ generated by the conjugate unit $\mathfrak{O}$. Under the polar specialization $(\mathfrak{E}, \mathfrak{O}) \mapsto(P i, P a)$, this becomes the eupolar structure, seemingly much simpler than the general eumonogenous structure $^{1}$ but in fact isomorphic to it. Eupolars can therefore serve as a prop for the intuition as well as a vehicle for simple proofs.

Within its self-assigned limits (eupolars and monogenous flexion structures), our paper deals with two sorts of questions-some clearly and provenly essential, others at first sight gratuitous but, we suspect, potentially of equal relevance. Let us explain.

\footnotetext{
${ }^{1}$ Meaning the structure generated under all flexion operations by a given flexion unit. Monogenous structures generated by an arbitrary element of $B I M U_{1}$ are of course more complex. For two equivalent characterizations of flexion units, in particular $P a$ and $P i$, see Section 17.12 below. As for the (unary or binary) flexion operations allowed in the generative process, they can all be constructed from the four elementary flexions $\lceil\rfloor, L$,$\rceil in proper association. They include all operations listed in Sections 17.2 -17.5 with the sole$ exceptions of swap and pus (push is allowed).
} 
The essential part revolves around the eupolar bisymmetral pair $\mathrm{pal}^{\bullet} / \mathrm{pil}^{\bullet}$ and its mirror image, the somewhat less important bisymmetrals $\operatorname{par}^{\bullet} /$ pir $^{\bullet}$. The first pair is doubly relevant to multizeta theory: firstly, because, together with its trigonometric counterpart $\mathrm{tal}^{\bullet} / \mathrm{til} \mathrm{l}^{\bullet}$, it goes into the making of the first factor $\mathrm{Zag}_{\mathrm{I}}^{\bullet} / \mathrm{Zig}_{\mathrm{I}}^{\bullet}$ in the classical trifactorization of the fundamental bimould $\mathrm{Zag} \bullet / \mathrm{Zig} \bullet$ that "carries all multizetas"; and secondly because it enters into the construction of the so-called singulators, themselves key to the study of the canonical multizeta irreducibles.

The pair $p a l^{\bullet} / \operatorname{pil}^{\bullet}$, as also $\operatorname{par}^{\bullet} /$ pir $^{\bullet}$, had already been dealt with in our previous papers, but somewhat desultorily, on a piecemeal basis. So a unified treatment, complete with motivations, definitions, characterizations, and proofs, was long overdue. The Sections 2-8 offer just such a treatment and, as is so often the case, systematization brings its own rewards. Thus, we exhibit two series, unsurpassed for simplicity, of alternals $\left\{\mathfrak{l} \mathfrak{e}_{r}^{\bullet}\right\}$ and $\left\{\mathfrak{r} \mathfrak{e}_{r}^{\bullet}\right\}$, and show that they are connected respectively to $\mathrm{pal}^{\bullet}$ and $\mathrm{pil}^{\bullet}$, as the ingredients of the $m u$-dilator $d u p a l^{\bullet}$ of $p a l^{\bullet}$ and the gari-dilator dipil $l^{\bullet}$ of pil $^{\bullet}$. This is a deeply satisfying state of affairs: it not only restores the symmetry (somewhat impaired in the previous approaches) between the co-equal swappees $\mathrm{pal}^{\bullet}$ and $\mathrm{pil}^{\bullet}$ but also leads to a simple proof of their bisymmetrality-of all extant proofs, the shortest. Nor do the pleasant surprises stop there. We introduce two additional series of alternals $\left\{\mathfrak{h} \mathfrak{e}_{r}^{\bullet}\right\}$ and $\left\{\mathfrak{k} \mathfrak{e}_{2 r}^{\bullet}\right\}$, less elementary than the first pair but still capable of a simple, transparent description, and show that these, too, are closely related to ripal $^{\bullet}$ (the gari-inverse of pal ${ }^{\bullet}$ ) and its even factor ripal $_{\mathrm{ev}}^{\bullet}$. It is truly gratifying to see that our four elementary or semi-elementary series of alternals (so far the only of their kind, i.e., the only ones known to admit a simple description) turn out to be, each in its own way, intimately interwoven with the central bisymmetrals.

The paper's second part, from Section 9 onwards, deals with the eupolar structure per se, without immediate applications in mind. The main challenge here is to generate, describe, and classify all regular, i.e., neg-invariant bisymmetrals and bialternals. Now, unlike the central bisymmetrals $\mathrm{pal}^{\bullet} / \mathrm{pil}^{\bullet}$ and $\mathrm{par}^{\bullet} / \mathrm{pir}^{\bullet}$, which are irregular (in the sense of being invariant under neither neg nor pari but only under the product pari o neg), the regular bisymmetrals $S a^{\bullet} / S^{\bullet}$ (as elements of GARI) correspond oneto-one to the regular bialternals (as elements of $A R I$ ) via the exponentiation expari from $A R I$ to $G A R I .^{2}$ So the attention now shifts to the bialternals which, living as they do in an algebra, are much easier to handle than the bisymmetrals. Starting from the two central-irregular pairs $\mathrm{pal}^{\bullet} / \mathrm{pil}^{\bullet}$ and $\mathrm{par}^{\bullet} / \mathrm{pir}^{\bullet}$, we describe two distinct procedures for producing two infinite series of bialternals, which in turn generate two distinct bialternal subalgebras of ARI. These two subalgebras do not coincide but partly overlap-though how far is yet unclear. Nor do we know whether, between themselves, they generate all bialternals.

\footnotetext{
${ }^{2}$ The much simpler correspondance between GARI-elements and their various dilators, though extremely useful, does not respect double symmetries, but merely turns symmetrality into alternality.
} 
This ignorance is galling. It is true that at the moment the polar bialternals, unlike the central bisymmetrals, ${ }^{3}$ have no known applications to multizeta algebra. But this may change. It would indeed be strange if the eupolar structure, even in its most recondite aspects, did not have some bearing on the study on multizetas. On the contrary, there is every reason to believe, and past experience strongly suggests, that most difficulties, irregularities or anomalies besetting multizeta theory ${ }^{4}$ originate in the eupolar domain which, being itself purely singular, holds the key to all the "singularity" scattered over the wider flexion field. Be that as it may, and all applications aside, the eupolar structure is a fascinating subject in its own right and deserves to be studied for its own sake.

So how are we to advance our knowledge of polar bialternals? Paradoxically, by widening the search: instead of obsessing about the sole bialternals and the spaces $A R I_{r}^{\mathrm{al}} / \underline{\mathrm{al}}=$ $A R I_{r}^{(1,1)}$ spanned by them, we may relax the notion and consider the larger spaces $A R I_{r}^{\left(d_{1}, d_{2}\right)}$ spanned by all eupolars of a (suitably defined) bialternality codegree $\left(d_{1}, d_{2}\right)$. The new approach embraces all eupolars, since for $\left(d_{1}, d_{2}\right)$ large enough ${ }^{5} A R I_{r}^{\left(d_{1}, d_{2}\right)}$ coincides with the whole of ARI. Moreover, the dimensions

$$
\text { Bial }_{r}^{d_{1}, d_{2}}:=\operatorname{dim}\left(A R I_{r}^{\left(d_{1}, d_{2}\right)}\right)
$$

or rather the differences

$$
\operatorname{bial}_{r}^{d_{1}, d_{2}}:=\mathrm{Bial}_{r}^{d_{1}, d_{2}}-\mathrm{Bial}_{r}^{d_{1}-1, d_{2}}-\mathrm{Bial}_{r}^{d_{1}, d_{2}-1}+\mathrm{Bial}_{r}^{d_{1}-1, d_{2}-1}
$$

which constitute the entries of the so-called bialternality grid, seem to follow a remarkable pattern. In particular, when we add the quite natural requirement of push-invariance, every second grid entry vanishes, leading to the so-called bialternality chessboard.

The corresponding computations, however, are extremely complex and progress only haltingly. At the moment, we are stuck at length $r=8$ : enough to discern the outlines of a tantalizing pattern; not enough to see the full picture emerge. The investigation goes on but it may be quite some time before the next batch of data arrives. ${ }^{6}$ So, rather than delay indefinitely the paper's publication, we have chosen to let the matter stand there-for now. Should fresh results complete the picture, they will be posted on our (regularly updated) homepage.

The present draft also contains two sizeable additions: Section 15, which shows what sort of changes the bialternality grid and chessboard undergo when we move on to polynomialvalued bimoulds; and Section 16, which (pending a systematic treatment in [5]) sketches the sort of complications attendant upon the passage from polar to trigonometric bisymmetrals. We wind up with Section 17, which recalls the main definitions about flexion

\footnotetext{
${ }^{3}$ And, of course, unlike the polynomial bialternals.

${ }^{4}$ Like, for example, the existence of the exceptional, polynomial-valued bialternals $\operatorname{carma} \bullet^{\bullet}$ carmi $\bullet$. See E1 and E2.

${ }^{5} d_{1}+d_{2}>r$ suffices.

${ }^{6}$ With many flexion operations, especially when working in algebras, it does not take much computational power to reach even length $r=20$. With others, such as inflected group inversion, inflected exponentiation or, like in the present instance, when it comes to expressing that a bimould has a given bialternality codegree, difficulties arise much earlier.
} 
theory, and Section 18, which gives short Maple programs for generating some of the main objects discussed in the paper. Lastly, numerous illustrative tables have been posted on our homepage. $^{7}$

\subsection{Conceptual vs Mechanical Proofs. The Priorities of Exploration}

The sheer profusion of formulae in flexion theory makes it strictly impossible to write down regular proofs for each of them. Clearly, identities involving such key bimoulds as pal ${ }^{\bullet}$ pil $\iota^{\bullet}$ deserve to be established with care, to do justice to the centrality and flagship quality of these objects. But what about the common run of flexion formulae? For them, it would be nice (time-saving and reassuring) to be able to fall back on a

Mechanical truth criterion (conjectural):

Any bimould-valued flexion identity of the form

$$
\mathcal{R} \bullet\left(F_{1}, \ldots, F_{p} ; A_{1}^{\bullet}, \ldots, A_{q}^{\bullet}\right) \equiv 0 \text { with } F_{i} \in \text { FLEXIONS }, A_{j}^{\bullet} \in \mathrm{BIMU}
$$

of total depth d

$$
d=\operatorname{depth}\left(\mathcal{R}^{\bullet}\right):=\sum_{i} \operatorname{depth}\left(F_{i}\right)+\sum_{j} \operatorname{depth}\left(A_{j}^{\bullet}\right)
$$

is automatically true for all lengths $r$ as soon as it holds identically for all arguments $A_{j}^{\bullet}$ and all lengths $r \leq d+1$.

This of course would require that we properly define the partial depths in formula (2).

The depth of "products" $F_{i}$ (associative or pre-Lie) would be 1; that of "alternate" operations (commutators, Lie brackets etc.) would be 2; and that of complex operations like the singulators would probably have to be 3 or 4 .

The depth of the arguments $A_{j}^{\bullet}$ would be 1 when $A_{j}^{\bullet}$ is allowed to range unrestrained over $B I M U$; or 2 if when $A_{j}^{\bullet}$ ranges over the set of all bimoulds with a simple symmetry; or again 3 or 4 if when it ranges over all bimoulds with a regular double symmetry.

Though the existence of some such truth criterion would seem almost certain, none has been established as yet. On the other hand, in the identities commonly encountered in flexion theory the total depth $d$, summarily assessed along the above lines, rarely exceeds 6 or 7 . So we may make safety doubly or trebly safe by verifying our identities up to the length $2 d$ or $3 d$ instead of $d+1$, which remains well within the range of the computationally feasible, and if the identities pass the test, confidently assume their validity.

But there is a catch here: in many important instances, the arguments $A_{j}^{\bullet}$ do not range over a vast enough domain of BIMU. For instance, the irregular (though central!) bisymmetrals $\mathrm{pal}^{\bullet} / \mathrm{pil}^{\bullet}$ are fairly '1isolated" creatures, unlike the regular $^{8}$ (though less central!) bisymmetrals $\mathrm{Sa}^{\bullet} / \mathrm{Si}^{\bullet}$. For the likes $\mathrm{pal} \boldsymbol{\iota}^{\bullet} / \mathrm{pil}^{\bullet}$ or $\mathrm{par}^{\bullet} / \mathrm{pir}^{\bullet}$, therefore, no "mechanical truth criterion" would work, and there is no way we can dispense with regular proofs here.

\footnotetext{
${ }^{7}$ at http://www.math.u-psud.fr/ ecalle/flexion.html.

8 i.e., neg-invariant.
} 
That said, careful consolidation, essential in the central, vital parts of an evolving theory, is one thing, and unfettered exploration, normal and legitimate at the fringes of the theory, is another. Each has its own logic, norms, and imperatives, and it would be foolish to mix up the two.

\subsection{Lie or Pre-lie Brackets and Group Laws. Anti-actions}

This first paragraph is there simply to dispel possible misconceptions about the flexion laws, the corresponding anti-actions, and the impact on these of the basic involution swap, which is the very glue of dimorphy.

First, we have the overarching structure AXI/GAXI, whose elements are bimould pairs $\mathcal{A}^{\bullet}=\left(\mathcal{A}_{L}^{\bullet}, \mathcal{A}_{R}^{\bullet}\right)$. Then we have the unary structures (seven in number, up to isomorphism) consisting of simple bimoulds $A^{\bullet}$ and corresponding to as many substructures of AXI/GAXI, each one of which is defined by a simple linkage $\mathcal{A}_{R}^{\bullet} \equiv h . \mathcal{A}_{L}^{\bullet}$ between left and right components. The number of suitable $h$ is of course very limited, and those $h$ that act on the algebras turn out to be involutions in six of the seven possible cases.

Let A/I/GA/I be such a unary structure ${ }^{9}$; let I/A/GI/A be the mirror structure under swap; and let $h_{1}, h_{2}, h_{3}, h_{4}$ be the four corresponding involutions

$$
\begin{array}{r}
\text { a/i } \longrightarrow h_{1} ; \quad \text { ifa } \longrightarrow h_{2} ; \\
\text { ga/i } \longrightarrow h_{3} ; \text { gi } \int \mathrm{a} \longrightarrow h_{4} .
\end{array}
$$

The laws are simply derived from the overstructure AXI/GAXI

$$
\begin{aligned}
& \operatorname{prea}\left(A^{\bullet}, B^{\bullet}\right)=\operatorname{preaxi}\left(\mathcal{A}_{1}^{\bullet}, \mathcal{B}_{1}^{\bullet}\right) ; \operatorname{prei} \int \mathrm{a}\left(A^{\bullet}, B^{\bullet}\right)=\operatorname{preaxi}\left(\mathcal{A}_{2}^{\bullet}, \mathcal{B}_{2}^{\bullet}\right) \text {; } \\
& \mathrm{a} / \mathrm{i}\left(A^{\bullet}, B^{\bullet}\right)=\operatorname{axi}\left(\mathcal{A}_{1}, \mathcal{B}_{1}\right) ; \quad \mathrm{i} \int \mathrm{a}\left(A^{\bullet}, B^{\bullet}\right)=\operatorname{axi}\left(\mathcal{A}_{2}, \mathcal{B}_{2}\right) ; \\
& \operatorname{ga} \int \mathrm{i}\left(A^{\bullet}, B^{\bullet}\right)=\operatorname{gaxi}\left(\mathcal{A}_{3}^{\bullet}, \mathcal{B}_{3}^{\bullet}\right) ; \quad \operatorname{gi} \int \mathrm{a}\left(A^{\bullet}, B^{\bullet}\right)=\operatorname{gaxi}\left(\mathcal{A}_{4}^{\bullet}, \mathcal{B}_{4}^{\bullet}\right)
\end{aligned}
$$

with

$$
\begin{aligned}
& \mathcal{A}_{i, L}^{\bullet}:=A^{\bullet} ; \mathcal{A}_{i, R}^{\bullet}:=h_{i} . A^{\bullet} \quad(\forall i \in\{1,2,3,4\}), \\
& \mathcal{B}_{i, L}^{\bullet}:=B^{\bullet} ; \mathcal{B}_{i, R}^{\bullet}:=h_{i} . A^{\bullet} \quad(\forall i \in\{1,2,3,4\}) .
\end{aligned}
$$

The anti-actions also are similarly defined

$$
\begin{aligned}
\operatorname{a} / \operatorname{it}\left(A^{\bullet}\right) & =\operatorname{axit}\left(\mathcal{A}_{1}^{\bullet}\right) ; \operatorname{ij} \operatorname{at}\left(A^{\bullet}\right)=\operatorname{axit}\left(\mathcal{A}_{2}^{\bullet}\right) ; \\
\operatorname{ga} / \operatorname{it}\left(A^{\bullet}\right) & =\operatorname{gaxit}\left(\mathcal{A}_{3}^{\bullet}\right) ; \operatorname{gi} / \operatorname{at}\left(A^{\bullet}\right)=\operatorname{gaxit}\left(\mathcal{A}_{4}^{\bullet}\right)
\end{aligned}
$$

but whereas under the vowel swap $a \leftrightarrow i$ the three types of laws (pre-Lie, Lie, or associative) transmute into one another

$$
\begin{aligned}
& \left.\operatorname{prei} \int \mathrm{a}\left(A^{\bullet}, B^{\bullet}\right)=\text { swap.prea/i(swap. } \mathrm{A}^{\bullet}, \text { swap. } B^{\bullet}\right) \text {, } \\
& \mathrm{i} f \mathrm{a}\left(A^{\bullet}, B^{\bullet}\right)=\quad \text { swap.a } \mathrm{i}\left(\operatorname{swap} . \mathrm{A}^{\bullet} \text {, swap. } B^{\bullet}\right) \text {, } \\
& \text { gifa }\left(A^{\bullet}, B^{\bullet}\right)=\text { swap.ga } \int \mathrm{i}\left(\text { swap. } \mathrm{A}^{\bullet} \text {, swap. } B^{\bullet}\right) \text {, }
\end{aligned}
$$

the corresponding anti-actions do not relate in this way

$$
\begin{aligned}
\text { if } \int \operatorname{at}\left(A^{\bullet}\right) & \left.\neq \text { swap.a/it(swap. } A^{\bullet}\right) \text {.swap, } \\
\operatorname{gi} \int \operatorname{at}\left(A^{\bullet}\right) & \left.\neq \text { swap.ga/it(swap. } A^{\bullet}\right) \text {.swap }
\end{aligned}
$$

and clearly cannot, since the right-hand sides (above) fail to define a $m u$-derivation, resp. a $m u$-isomorphism.

\footnotetext{
${ }^{9}$ With the unusual mid-letter $\int$ (pronounced sh) suggesting generality.
} 
Nonetheless, the laws may be expressed in terms of the anti-actions. Thus for the first law we have

$$
\begin{aligned}
\operatorname{prea} \int \mathrm{i}\left(\mathrm{A}^{\bullet}, B^{\bullet}\right) & =\operatorname{ajit}\left(B^{\bullet}\right) \cdot A^{\bullet}+\operatorname{mu}\left(A^{\bullet}, B^{\bullet}\right), \\
\operatorname{aji}\left(A^{\bullet}, B^{\bullet}\right) & =\operatorname{prea} \int \mathrm{i}\left(A^{\bullet}, B^{\bullet}\right)-\operatorname{prea} \int \mathrm{i}\left(B^{\bullet}, A^{\bullet}\right) \\
& =\operatorname{ajit}\left(B^{\bullet}\right) \cdot A^{\bullet}-\operatorname{ajit}\left(A^{\bullet}\right) \cdot B^{\bullet}+\operatorname{lu}\left(A^{\bullet}, B^{\bullet}\right), \\
\operatorname{ga} /\left(A^{\bullet}, B^{\bullet}\right) & =\operatorname{mu}\left(\operatorname{ga} \int \operatorname{it}\left(B^{\bullet}\right) \cdot A^{\bullet}, B^{\bullet}\right) .
\end{aligned}
$$

Of course, the same identities hold with " $a \int i$ " changed everywhere to "ifa".

\subsection{Left-right Separation}

The phenomenon is summed up by the following identities, which speak for themselves

$$
\begin{aligned}
\operatorname{axit}\left(\mathcal{A}^{\bullet}\right) & =\operatorname{amit}\left(\mathcal{A}_{L}^{\bullet}\right)+\operatorname{anit}\left(\mathcal{A}_{R}^{\bullet}\right), \\
\operatorname{gaxit}\left(\mathcal{A}^{\bullet}\right) & =\operatorname{gamit}\left(\mathcal{A}_{L}^{\bullet}\right) \cdot \operatorname{ganit}\left(\left(\operatorname{gamit}\left(\mathcal{A}_{L}^{\bullet}\right)\right)^{-1} \mathcal{A}_{R}^{\bullet}\right) \\
& =\operatorname{ganit}\left(\mathcal{A}_{R}^{\bullet}\right) \cdot \operatorname{gamit}\left(\left(\operatorname{ganit}\left(\mathcal{A}_{R}^{\bullet}\right)\right)^{-1} \mathcal{A}_{L}^{\bullet}\right) .
\end{aligned}
$$

The last two identities are easier to check in the following, equivalent form:

$$
\begin{gathered}
\operatorname{gamit}\left(A^{\bullet}\right) \cdot \operatorname{ganit}\left(B^{\bullet}\right)=\operatorname{gaxit}\left(\mathcal{C}^{\bullet}\right) \quad \text { with } \quad \mathcal{C}_{L}^{\bullet}:=A^{\bullet}, \mathcal{C}_{R}^{\bullet}:=\operatorname{gamit}\left(A^{\bullet}\right) . B^{\bullet}, \\
\operatorname{ganit}\left(A^{\bullet}\right) \cdot \operatorname{gamit}\left(B^{\bullet}\right)=\operatorname{gaxit}\left(\mathcal{D}^{\bullet}\right) \quad \text { with } \mathcal{D}_{L}^{\bullet}:=\operatorname{ganit}\left(A^{\bullet}\right) . B^{\bullet}, \mathcal{D}_{R}^{\bullet}:=A^{\bullet} .
\end{gathered}
$$

\subsection{Closure Under the Basic Involution Swap}

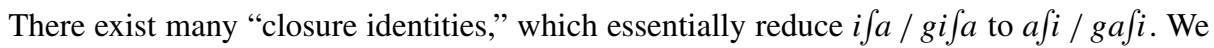
mention the only one that we shall really require

$$
\operatorname{gira}\left(A^{\bullet}, B^{\bullet}\right) \equiv \operatorname{ganit}\left(\operatorname{rash} . B^{\bullet}\right) \cdot \operatorname{gari}\left(A^{\bullet}, \operatorname{ras} . B^{\bullet}\right)
$$

with

$$
\begin{aligned}
\operatorname{rash} . B^{\bullet} & :=\operatorname{mu}\left(\text { push.swap.invmu.swap. } \mathrm{B}^{\bullet}, B^{\bullet}\right), \\
\operatorname{ras} . B^{\bullet} & :=\text { invgari.swap.invgari.swap. } \mathrm{B}^{\bullet} .
\end{aligned}
$$

\subsection{The Monogenous Algebra Flex(E). Basis and Projectors}

The monogenous algebra Flex $(\mathfrak{E})=\oplus$ Flex $_{r}(\mathfrak{E})$ was constructed in [4] Sections 3 and 4, along with the standard basis $\left\{\mathfrak{e}_{t}^{\bullet}\right\} \sim\left\{\mathfrak{e}_{t}^{\bullet}\right\}$ of Flex $(\mathfrak{E})$. That standard basis has cardinality $(2 r) ! /(r !(r+1) !)$ and admits a natural indexation either by $r$-node binary trees $t$ or by some special $r$-term sequences $\underline{t}$ that stand in one-to-one correspondance with these trees. The basis elements are defined inductively

$$
\begin{aligned}
\mathfrak{e}_{t}^{\bullet} & :=\operatorname{amnit}\left(\mathfrak{e}_{t_{1}}^{\bullet}, \mathfrak{e}_{t_{2}}^{\bullet}\right) \cdot \mathfrak{E}^{\bullet} \quad \Longleftrightarrow \\
\mathfrak{e}_{t}^{w} & :=\mathfrak{e}_{t_{1}}^{\left.w^{1}\right\rfloor} \mathfrak{E}^{\left\lceil w_{\mathbf{i}}\right\rceil} \mathfrak{e}_{t_{2}}^{\left\lfloor w^{2}\right.} \quad \text { with } \quad w=w^{\mathbf{1}} \cdot w_{\mathbf{i}} \cdot w^{\mathbf{2}} \quad \text { and } \quad r_{1}+r_{2}=r-1
\end{aligned}
$$

and the corresponding inductions for trees and sequences go like this

$$
\begin{aligned}
&\left(t_{1}, t_{2}\right) \mapsto t:=\left\{t_{1} \leftarrow \bullet \rightarrow t_{2}\right\}, \\
&\left(\underline{t_{1}}, \underline{t_{2}}\right) \mapsto \underline{t}:=\left[\underline{\boldsymbol{t}}_{1}, r_{1}+1, \underline{\boldsymbol{t}}^{\left(r_{1}+1\right)}\right] .
\end{aligned}
$$

Here, $\left\{t_{1} \leftarrow \bullet \rightarrow t_{2}\right\}$ denotes of course the binary tree we get by glueing $t_{1}$ (resp. $t_{2}$ ) to the root-node $\bullet$ as its left (resp. right) branch. On the sequence side, $r_{1}$ denotes the length 
of $\underline{\boldsymbol{t}}_{\mathbf{1}}$ and $\underline{\boldsymbol{t}}^{\left(r_{1}+1\right)}$ results from $\underline{\boldsymbol{t}}_{\mathbf{2}}$ by adding $r_{1}+1$ to its every element, after which we concatenate everything, thus producing a sequence $\underline{t}$ that is some well-defined permutation of $[1,2, \ldots, r]$.

What we now need is an algorithm for projecting the general element $X^{\bullet}$ of Flex $_{r}(\mathfrak{E})$ onto the standard basis. The following formula does just that

$$
X^{\bullet} \equiv \sum_{t} \mathfrak{e}_{t}^{\bullet} \operatorname{Res}^{t} X^{\bullet} \stackrel{\text { i.e. }}{=} \sum \mathfrak{e}_{\left[i_{1}, \ldots, i_{r}\right]}^{\bullet} \operatorname{Res}^{i_{1}, \ldots, i_{r}} X^{\bullet}
$$

with projectors $\operatorname{Res}^{i_{1}, \ldots, i_{r}}$ capable of two interpretations

(i) $\operatorname{Res}^{i_{1}, \ldots, i_{r}}:=\operatorname{Res}_{u_{i_{r}}} \ldots \operatorname{Res}_{u_{i_{2}}} \operatorname{Res}_{u_{i_{1}}}$,

(ii) $\operatorname{Res}^{i_{1}, \ldots, i_{r}}:=\operatorname{Res}_{v_{i_{1}}} \cdot \operatorname{Res}_{v_{i_{2}}} \ldots \operatorname{Res}_{v_{i_{r}}}$.

Mark the order inversion from (i) to (ii). To calculate $\operatorname{Res}_{u_{i}} X^{\bullet}$, we set all variables $v_{i}$ equal to 0 ; then take the coefficient of $\mathfrak{E}^{\left(\begin{array}{c}u_{i} \\ 0\end{array}\right)}$ minus ${ }^{10}$ the coefficient of $\left.\mathfrak{E}^{\left(-u_{i}\right.}{ }_{0}\right)$; then set $u_{i}=0$. Performing the operation $r$ times, successively with $\operatorname{Res}_{u_{i_{1}}}, \operatorname{Res}_{u_{i_{2}}}$ etc., we end up with a scalar that does not depend on the particular expression chosen for $X^{\bullet}$ (elements of Flex $_{r}(\mathfrak{E})$, we recall, admit many different expressions).

To calculate $\operatorname{Res}_{v_{i}} X^{\bullet}$, we go through exactly the same motions, but with the roles of the $u_{i}$ 's and $v_{i}$ 's exchanged and the order of the operations reversed. Once again, the final result does not depend on the expression ${ }^{11}$ of $X^{\bullet}$, and coincides with the result of the first procedure.

Clearly, in the polar specialization $\mathfrak{E}=P a($ resp. $P i)$, the operator $\operatorname{Res}_{u_{i}}\left(\right.$ resp. $\left.\operatorname{Res}_{v_{i}}\right)$ corresponds to the taking of the residue at $u_{i}=0$ (resp. $\left.v_{i}=0\right)$.

\subsection{Dilators: what are they, and what are they Good for?}

Infinitesimal generators and dilators have this in common that they often permit to rephrase problems about groups as more tractable problems about algebras. But of the two, the dilators are the more useful by far, mainly because they are so much closer, conceptually and computationally, to the group elements from which they derive.

Here is how the inflected dilators $d i S^{\bullet}$ and $d a S^{\bullet}$ and the uninflected dilator $d u S^{\bullet}$ relate to the corresponding group element $S^{\bullet}$ (henceforth referred to as the dilatee)

$$
\begin{array}{lr}
\operatorname{der} . S^{\bullet}=\operatorname{preari}\left(S^{\bullet}, \text { di } S^{\bullet}\right) & \left(\operatorname{di} S^{\bullet}=\text { gari }- \text { dilator }\right), \\
\operatorname{der} . S^{\bullet}=\operatorname{preira}\left(S^{\bullet}, d a S^{\bullet}\right) & \left(\operatorname{da} S^{\bullet}=\text { gira }- \text { dilator }\right), \\
\text { dur. } S^{\bullet}=\operatorname{mu}\left(S^{\bullet}, d u S^{\bullet}\right) & \left(\operatorname{du} S^{\bullet}=\mathrm{mu}-\text { dilator }\right) .
\end{array}
$$

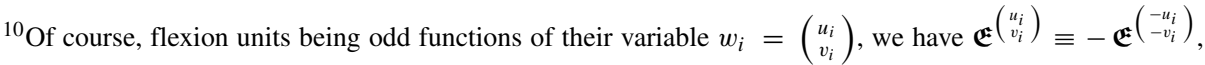
but since complex superpositions of flexion operations are liable to yield either form, both possibilities must be taken into account.

${ }^{11}$ Elements of Flex (E) can be expressed/expanded in numerous, outwardly distinct ways and, when resulting from a sequence of flexion operations, they usually appear, prior to simplification, in an absurdly complicated shape.
} 
The three relations are entirely parallel: indeed, the Lie bracket corresponding to $m u$ is $l u$ and $m u$ may (trivially) be regarded as a pre-Lie bracket prelu for $l u$. As for the operators $d e r$ and $d u r$, they are $m u$-derivations each

$$
\begin{aligned}
\operatorname{der} . S^{w_{1}, \ldots, w_{r}} & :=r S^{w_{1}, \ldots, w_{r}}, \\
\operatorname{dur} . S^{w_{1}, \ldots, w_{r}} & :=\left(u_{1}+\ldots u_{r}\right) S^{w_{1}, \ldots, w_{r}} .
\end{aligned}
$$

In the context of the monogenous structures Flex $_{r}(\mathfrak{E})$, the latter derivation dur is particularly relevant when $\mathfrak{E}=P a$ but even then it has the slight drawback of taking us out of Flex $(\mathfrak{E})$ into something which, with due quotation marks, might be called "Flex $(\mathfrak{E}) \otimes\left\{I^{\bullet}\right\}$," with an elementary $I^{\bullet}$ that is 1 or 0 according as the length $r(\bullet)$ is 1 or not. $^{12}$

To remedy the non-internal character of $d u r$, we must sometimes replace it by duur, which is a bona fide internal $m u$-derivation of Flex(E) into itself. Since all elements of Flex $_{r}(\mathfrak{E})$ may be expressed ${ }^{13}$ as a superposition of terms $M_{r}^{\bullet}$ of the form

$$
M_{r}^{\bullet}:=\operatorname{amnit}\left(M_{r_{1}}^{\bullet}, M_{r_{2}}^{\bullet}\right) \cdot \mathfrak{E}^{\bullet} \quad \text { with } \quad r_{1}+r_{2}=r-1 \text { and } M_{r_{i}}^{\bullet} \in \text { Flex }_{r_{i}}(\mathfrak{E}) \text {, }
$$

it is enough to say how duur acts on these $M_{r}^{\bullet}$, and here is how it acts

$$
\operatorname{duur} . M_{r}^{\bullet}:=\operatorname{mu}\left(M_{\mathrm{r}_{1}}^{\bullet}, \mathrm{I}^{\bullet}, \mathrm{M}_{r_{2}}^{\bullet}\right) \text {. }
$$

The corresponding dilator relation then assumes the form

$$
\operatorname{duur} . S^{\bullet}=\operatorname{mu}\left(S^{\bullet}, \operatorname{duur} . \operatorname{duu} S^{\bullet}\right)
$$

or the equivalent form

$$
S^{\bullet}=\operatorname{muu}\left(S^{\bullet}, \operatorname{duu} S^{\bullet}\right)
$$

with тии denoting a sort of integration-by-part operator but with the twist that the underlying product $m u$ is non-commutative

$$
\operatorname{muu}\left(A^{\bullet}, B^{\bullet}\right) \stackrel{\text { essentially }}{:=} \operatorname{duur}{ }^{-1} \cdot \operatorname{mu}\left(A^{\bullet}, \operatorname{duur} . B^{\bullet}\right)
$$

or more rigorously

$$
\operatorname{muu}\left(A^{\bullet}, B^{\bullet}\right):=\operatorname{amnit}\left(\operatorname{mu}\left(A^{\bullet}, B_{1}^{\bullet}\right), B_{2}^{\bullet}\right) \mathfrak{E}^{\bullet} \quad \text { if } \quad B^{\bullet}=\operatorname{amnit}\left(B_{1}^{\bullet}, B_{2}^{\bullet}\right) \cdot \mathfrak{E}^{\bullet}
$$

\subsection{Relations Between Inflected and Non-inflected Dilators}

For any $S^{\bullet}$ such that $S^{\emptyset}=1$, the inflected dilators $d i S^{\bullet}, d a S^{\bullet}$ and the non-inflected dilator $d u S^{\bullet}$ relate according to

$$
\begin{aligned}
& \text { der.duS } S^{\bullet}-\operatorname{dur} \cdot \operatorname{di} S^{\bullet}+\operatorname{lu}\left(\operatorname{di} S^{\bullet}, \operatorname{du}^{\bullet}\right)-\operatorname{arit}\left(\operatorname{dis} S^{\bullet}\right) \cdot \operatorname{du} S^{\bullet}=0 \text {, } \\
& \text { der.duS } S^{\bullet}-\operatorname{dur} \cdot \operatorname{da} S^{\bullet}+\operatorname{lu}\left(\operatorname{da} S^{\bullet}, \operatorname{du}^{\bullet}\right)-\operatorname{irat}\left(\operatorname{da} S^{\bullet}\right) \cdot \operatorname{du} S^{\bullet}=0 \text {. }
\end{aligned}
$$

\footnotetext{
${ }^{12} I^{\bullet}$ is the unit for mould composition $\circ$ and should be carefully distinguished from the multiplication unit $1^{\bullet}$ which is 1 or 0 according as the length $r(\bullet)$ is 0 or $>0$.

${ }^{13}$ See [4], (3.35).
} 
The shortest way to prove (26), (27) is to rewrite the dilator identities (17), (18), (19) as follows $^{14}$

$$
\begin{aligned}
& \mathrm{D}_{1} \cdot S^{\bullet}=\operatorname{mu}\left(\mathrm{S}^{\bullet}, \operatorname{di} \mathrm{S}^{\bullet}\right) \text { with } \mathrm{D}_{1}:=\operatorname{der}-\operatorname{arit}\left(\operatorname{di} S^{\bullet}\right) \\
& \mathrm{D}_{2} \cdot S^{\bullet}=\operatorname{mu}\left(\mathrm{S}^{\bullet}, \operatorname{da} S^{\bullet}\right) \text { with } \mathrm{D}_{2}:=\operatorname{der}-\operatorname{irat}\left(\operatorname{da} S^{\bullet}\right) \\
& \mathrm{D}_{3} \cdot S^{\bullet}=\operatorname{mu}\left(\mathrm{S}^{\bullet}, \operatorname{du} S^{\bullet}\right) \text { with } \mathrm{D}_{3}:=d u r
\end{aligned}
$$

and to observe that since the derivation $d u r$ commutes with all three derivations der, $\operatorname{arit}\left(\operatorname{diS}^{\bullet}\right)$, irat $\left(\operatorname{daS}^{\bullet}\right)$, we have

$$
\left[\mathrm{D}_{1}, \mathrm{D}_{3}\right]=\left[\mathrm{D}_{2}, \mathrm{D}_{3}\right]=0 \quad\left(\text { but }\left[\mathrm{D}_{1}, \mathrm{D}_{2}\right] \neq 0\right) \text {. }
$$

To establish (27), which we shall require in the sequel, we apply the commutator $\left[D_{2}, D_{3}\right]$ to $S^{\bullet}$. We get successively

$0=\mathrm{D}_{2} \cdot \mathrm{D}_{3} \cdot \mathrm{S}^{\bullet}-\mathrm{D}_{3} \cdot \mathrm{D}_{2} \cdot \mathrm{S}^{\bullet}$,

$0=\mathrm{D}_{2} \cdot \mathrm{mu}\left(\mathrm{S}^{\bullet}, \mathrm{du} \mathrm{S}^{\bullet}\right)-\mathrm{D}_{3} \cdot \mathrm{mu}\left(\mathrm{S}^{\bullet}, \mathrm{da} \mathrm{S}^{\bullet}\right)$,

$0=\operatorname{mu}\left(D_{2} \cdot S^{\bullet}, d u S^{\bullet}\right)+\operatorname{mu}\left(S^{\bullet}, D_{2} \cdot d u S^{\bullet}\right)-m u\left(D_{3} \cdot S^{\bullet}, \mathrm{da}^{\bullet}\right)-\operatorname{mu}\left(S^{\bullet}, D_{3} \cdot d a S^{\bullet}\right)$,

$0=\operatorname{mu}\left(S^{\bullet}, \operatorname{da}^{\bullet}, \mathrm{du}^{\bullet}\right)+\operatorname{mu}\left(S^{\bullet}, \mathrm{D}_{2} \cdot \operatorname{du} S^{\bullet}\right)-\operatorname{mu}\left(S^{\bullet}, \operatorname{du} S^{\bullet}, \operatorname{da} S^{\bullet}\right)-\operatorname{mu}\left(S^{\bullet}, \mathrm{D}_{3} \cdot \operatorname{da} S^{\bullet}\right)$.

Since we assumed $S^{\emptyset}=1$, our $S^{\bullet}$ is $m u$-invertible. So we may $m u$-divide the last identity by $S^{\bullet}$ on the left, and what we are left with is exactly the sought-after identity (27). The proof of (26) is entirely analogous.

We may note that since the relations (26) and (27) are of the form

$$
\begin{aligned}
& r(w) \cdot d u S^{w}=\|\boldsymbol{u}\| \cdot \operatorname{di} S^{w}+\text { earlier terms, } \\
& r(w) \cdot d u S^{w}=\|\boldsymbol{u}\| \cdot d a S^{w}+\text { earlier terms, }
\end{aligned}
$$

they clearly determine $d i S^{\bullet}$ and $d a S^{\bullet}$ in terms of $d u S^{\bullet}$ and vice versa.

We may also observe that since prelu $:=m u$ is, trivially, a pre-Lie law for the Lie law $l u$, the relation (26), (27) can be rewritten in the following, particularly harmonious form

$$
\begin{aligned}
& \text { dur.dis } S^{\bullet}+\operatorname{prelu}\left(\operatorname{du} S^{\bullet}, \operatorname{di} S^{\bullet}\right)=\operatorname{der} \cdot \operatorname{du} S^{\bullet}+\operatorname{preari}\left(\operatorname{di} S^{\bullet}, \operatorname{du} S^{\bullet}\right), \\
& \operatorname{dur} \cdot \operatorname{da} S^{\bullet}+\operatorname{prelu}\left(\operatorname{du} S^{\bullet}, \operatorname{da} S^{\bullet}\right)=\operatorname{der} \cdot d u S^{\bullet}+\operatorname{preira}\left(\operatorname{da} S^{\bullet}, \operatorname{du} S^{\bullet}\right) \text {. }
\end{aligned}
$$

Furthermore, although there exists no simple direct relation between the inflected dilators $d i S^{\bullet}$ and $d a S^{\bullet}$, there exists, interestingly, an indirect one, via the non-inflected $d u S^{\bullet}$.

\subsection{Dilatees in Terms of the Dilators}

One goes from a $m u$-dilator $d u S^{\bullet}$ or $d u u S^{\bullet}$ to the source element $S^{\bullet}$ (the "dilatee") via the identities

$$
\begin{aligned}
S^{\boldsymbol{w}} & =1^{\boldsymbol{w}}+\sum_{\boldsymbol{w}^{1} \ldots \boldsymbol{w}^{s}=w} \operatorname{Paj}^{\left|\boldsymbol{u}^{1}\right|, \ldots,\left|\boldsymbol{u}^{s}\right|} \mathrm{duS}^{\boldsymbol{w}^{1}} \ldots \mathrm{duS}^{\boldsymbol{w}^{s}} \\
S^{\bullet} & =1^{\bullet}+\sum_{r_{1}+\ldots r_{s}=r(\bullet)} \overrightarrow{\operatorname{muu}}\left(\operatorname{duu}_{r_{1}}^{\bullet}, \ldots, \operatorname{duu} S_{r_{s}}^{\bullet}\right)
\end{aligned}
$$

\footnotetext{
${ }^{14}$ It goes without saying that the notations $D_{1}, D_{2}, D_{3}$ are simply shorthand for their full expression as given in (28), (29), and (31). Whenever these operators occur in the subsequent identities of the present section, they should therefore be mentally replaced by their defining relations. No ambiguity there.
} 
with a symmetral mould $\mathrm{Paj}^{\bullet}$ defined by

$$
\mathrm{Paj}^{x_{1}, \ldots, x_{r}}:=\prod_{1 \leq i \leq r} \frac{1}{x_{1}+\cdots+x_{i}} .
$$

Similarly, one goes from a gari-dilator $\operatorname{di}^{\bullet}$ to the source $S^{\bullet}$ via the identity

$$
S^{\bullet}=\sum_{r_{1}+\ldots r_{s}=r(\bullet)} P a j^{r_{1}, \ldots, r_{s}} \underset{\text { preari }}{\longrightarrow}\left(d i S_{r_{1}}^{\bullet}, \ldots, d i S_{r_{s}}^{\bullet}\right)
$$

with the same auxiliary mould $P a j \bullet$ but differently indexed.

An analogous formula expresses the product $T^{\bullet}=\operatorname{gari}\left(R^{\bullet}, S^{\bullet}\right)$ in terms of the dilators $^{15}$

$$
T^{\bullet}=R^{\bullet}+S^{\bullet}+\sum_{r_{0}+\ldots r_{s}=r(\bullet)} \operatorname{Paj}^{r_{1}, \ldots, r_{s}} \underset{\text { preari }}{\longrightarrow}\left(R_{r_{0}}^{\bullet}, \operatorname{di} S_{r_{1}}^{\bullet}, \ldots, \text { di } S_{r_{s}}^{\bullet}\right) .
$$

Mark the absence of $r_{0}$ in $\mathrm{Paj}^{r_{1}, \ldots, r_{s}}$.

We may also, and often must, express the operators $\operatorname{garit}\left(S^{\bullet}\right)$ and $\operatorname{adari}\left(S^{\bullet}\right)$ in terms of $d i S^{\bullet}$

$$
\begin{aligned}
\operatorname{garit}\left(S^{\bullet}\right) & =\mathrm{id}+\sum_{\boldsymbol{r}_{1}+\ldots \boldsymbol{r}_{s}=\boldsymbol{r}(\bullet)} \operatorname{Paj}^{r_{1}, \ldots, r_{s}} \operatorname{arit}\left(\operatorname{di} S_{r_{s}}^{\bullet}\right), \ldots \operatorname{arit}\left(d i S_{r_{1}}^{\bullet}\right), \\
\operatorname{adari}\left(S^{\bullet}\right) & =\mathrm{id}+\sum_{\boldsymbol{r}_{1}+\ldots \boldsymbol{r}_{\boldsymbol{s}}=\boldsymbol{r}(\bullet)} P a j^{r_{1}, \ldots, r_{s}} \underline{\operatorname{ari}}\left(\operatorname{di} S_{r_{1}}^{\bullet}\right), \ldots \underline{\operatorname{ari}}\left(\operatorname{di} S_{r_{s}}^{\bullet}\right),
\end{aligned}
$$

where ari denotes the adjoint action of $A R I$ on itself. ${ }^{16}$ The indexation of the operators $\underline{\operatorname{ari}}\left(\operatorname{di}_{S_{r_{i}}}^{\bullet}\right)$ and $\operatorname{arit}\left(\operatorname{di}_{r_{i}}^{\bullet}\right)$ goes in opposite directions, but this should not come as a surprise, since adari defines an action (of GARI on ARI) and garit an anti-action (of GARI on BIMU).

\subsection{Some Other Dilator Identities}

How does the gari-product affect dilators? Like this

$$
\begin{aligned}
T^{\bullet} & =\operatorname{gari}\left(R^{\bullet}, S^{\bullet}\right) \quad \Longrightarrow \\
\operatorname{di} T^{\bullet} & =\operatorname{di} S^{\bullet}+\operatorname{adari}\left(S^{\bullet}\right)^{-1} \cdot \operatorname{di}^{\bullet} .
\end{aligned}
$$

Since according to (42), adari $\left(S^{\bullet}\right)^{ \pm 1}$ can also be expressed in terms of $d i S^{\bullet}$, the above identity amounts to a sort of Campbell-Hausdorff formula for the composition of garidilators. In the same vein, we must mention the conversion formulae between

(i) The dilator $d i S^{\bullet}$ of $S^{\bullet}$.

(ii) The dilator diriS $S^{\bullet}$ of $\operatorname{ri} S^{\bullet}:=\operatorname{inv} \operatorname{gari}\left(S^{\bullet}\right)$.

(iii) The infinitesimal generator $l i S^{\bullet}:=\log \operatorname{ari}\left(S^{\bullet}\right)$.

\footnotetext{
${ }^{15}$ Of course, on the right-hand side of (40), we must substitute for $S^{\bullet}$ the expansion (39) and do likewise with $T^{\bullet}$.

${ }^{16}$ i.e., $\underline{\operatorname{arit}}\left(A^{\bullet}\right) . B^{\bullet} \equiv \operatorname{ari}\left(A^{\bullet}, B^{\bullet}\right)$.
} 
The conversion $\operatorname{di}^{\bullet} \leftrightarrow \operatorname{diri}^{\bullet}$ is via the involutive formula

$$
\begin{aligned}
\operatorname{diri} S^{\bullet} & =\sum_{\mathbf{1} \leq \mathbf{s}} \sum_{\boldsymbol{w}^{\mathbf{1}} \ldots \mathbf{w}^{\mathbf{s}}=\mathbf{w}} \operatorname{Japaj}^{r\left(\boldsymbol{w}^{\mathbf{1}}\right), \ldots, r\left(\boldsymbol{w}^{\mathbf{s}}\right)} \underset{\operatorname{preari}}{\longrightarrow}\left(\operatorname{di} S^{\boldsymbol{w}^{\mathbf{1}}}, \ldots, \text { di } S^{\mathbf{w} \boldsymbol{w}^{\mathbf{s}}}\right) \\
& =\sum_{\mathbf{1} \leq \mathbf{s}} \frac{1}{\mathbf{S}_{\boldsymbol{w}^{\mathbf{1}} \ldots \boldsymbol{w}^{\mathbf{s}}=\boldsymbol{w}}} \operatorname{Japaj}^{r\left(\boldsymbol{w}^{\mathbf{1}}\right), \ldots, r\left(\boldsymbol{w}^{\mathbf{s}}\right)} \underset{\operatorname{ari}}{\longrightarrow}\left(\operatorname{di}^{\boldsymbol{w}^{\mathbf{1}}}, \ldots, \operatorname{di}^{\boldsymbol{w}^{\mathbf{s}}}\right)
\end{aligned}
$$

with an alternal mould $\operatorname{Japaj}^{\bullet}:=\operatorname{Compo}\left(\mathrm{Ja}^{\bullet}, \mathrm{Paj}^{\bullet}\right)$ defined as $\mathrm{Paj}^{\bullet}$ pre-composed by the elementary mould $J a^{x_{1}, \ldots, x_{r}}:=(-1)^{r} x_{1}$. Thus we get

$$
\text { Japaj }_{1}^{x}=1 ; \text { Japaj }^{x_{1}, x_{2}}=\frac{x_{1}-x_{2}}{x_{1} x_{2}} ; \text { Japaj }^{x_{1}, x_{2}, x_{3}}=\frac{x_{1} x_{3}-x_{1}^{2}+x_{2}^{2}-x_{3}^{2}}{x_{1} x_{3}\left(x_{1}+x_{2}\right)\left(x_{2}+x_{3}\right)} \text { etc. }
$$

The conversion $l i S^{\bullet} \rightarrow d i S^{\bullet}$ is via an even simpler formula

$$
\begin{aligned}
d i S^{\bullet} & =\sum_{1 \leq s} \sum_{w^{1} \ldots w^{s}=w} \operatorname{Bin}^{r\left(w^{1}\right), \ldots, r\left(w^{s}\right)} \underset{\operatorname{preari}}{\longrightarrow}\left(l i S^{w^{1}}, \ldots, l i S^{w^{s}}\right) \\
& =\sum_{1 \leq s} \frac{1}{s} \sum_{w^{1} \ldots w^{s}=w} \operatorname{Bin}^{r\left(w^{1}\right), \ldots, r\left(w^{s}\right)} \underset{\operatorname{ari}}{\longrightarrow}\left(l i S^{w^{1}}, \ldots, l i S^{w^{s}}\right)
\end{aligned}
$$

with an elementary alternal mould $\operatorname{Bin}^{\bullet}$ defined by

$$
\operatorname{Bin}^{x_{1}, \ldots, x_{r}}:=\frac{1}{r} \sum_{1 \leq j \leq r} \frac{x_{j}}{(j-1) !(r-j) !} .
$$

\subsection{Internals and Externals}

A bimould $A^{\bullet}$ is said to be internal if, for all $r$, it verifies two dual properties, which in short notation read

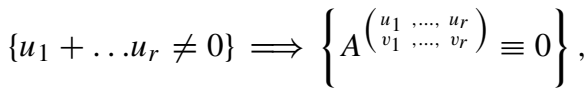

$$
\begin{aligned}
& \left\{v_{i}-v_{i}^{\prime}=\mathrm{const} ; \forall i\right\} \Longrightarrow\left\{A^{\left(\begin{array}{c}
u_{1}, \ldots, u_{r} \\
v_{1}, \ldots, v_{r}
\end{array}\right)} \equiv A^{\left(\begin{array}{c}
u_{1}, \ldots, u_{r} \\
v_{1}^{\prime}, \ldots, \\
v_{r}^{\prime}
\end{array}\right)}\right\}
\end{aligned}
$$

and in long notation assume the more natural form

$$
\begin{aligned}
& \left\{u_{0} \neq 0\right\} \quad \Longrightarrow\left\{A\left(\left[\begin{array}{c}
\left.u_{0}\right], u_{1}, \ldots, u_{r} \\
v_{0}
\end{array}\right], v_{1}, \ldots, v_{r}\right) \equiv 0\right\},
\end{aligned}
$$

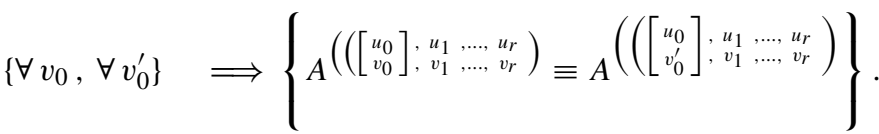

Internals constitute an ideal ARI intern of ARI, resp. a normal subgroup GARI $I_{\text {intern }}$ of GARI. The elements of the corresponding quotients are referred to as externals

$$
\begin{aligned}
\text { ARI }_{\text {extern }} & :=\text { ARI/ARI } \\
\text { GARI }_{\text {extern }} & :=\text { GARI} / G A R I_{\text {intern }} .
\end{aligned}
$$


Moreover, when restricted to internals, the ari bracket reduces, up to order, to the simpler $l u$ bracket, and the gari product, again up to order, reduces to the $m u$ product

$$
\begin{aligned}
\operatorname{ari}\left(A^{\bullet}, B^{\bullet}\right) \equiv \operatorname{lu}\left(B^{\bullet}, A^{\bullet}\right) & \forall A^{\bullet}, B^{\bullet} \in \mathrm{ARI}_{\text {intern }} \\
\operatorname{gari}\left(A^{\bullet}, B^{\bullet}\right) \equiv \operatorname{mu}\left(B^{\bullet}, A^{\bullet}\right) & \forall A^{\bullet}, B^{\bullet} \in \mathrm{GARI}_{\text {intern }}
\end{aligned}
$$

Lastly, we have two useful identities governing the action of internal bimoulds on general ones

$$
\begin{array}{cl}
\operatorname{arit}\left(A^{\bullet}\right) . B^{\bullet} \equiv \mathrm{lu}\left(A^{\bullet}, B^{\bullet}\right) & \forall A^{\bullet} \in \mathrm{ARI}_{\text {intern }}, \forall B^{\bullet} \in \mathrm{ARI}, \\
\operatorname{garit}\left(A^{\bullet}\right) . B^{\bullet} \equiv \operatorname{mu}\left(A^{\bullet}, B^{\bullet}\right) & \forall A^{\bullet} \in \mathrm{GARI}_{\text {intern }}, \forall B^{\bullet} \in \mathrm{GARI}
\end{array}
$$

and two anologous identites for the action of general bimoulds on internals

$$
\begin{array}{cl}
\operatorname{arit}\left(B^{\bullet}\right) . A^{\bullet} \equiv \operatorname{ari}\left(A^{\bullet}, B^{\bullet}\right) & \forall A^{\bullet} \in \mathrm{ARI}_{\text {intern }}, \forall B^{\bullet} \in \mathrm{ARI}, \\
\operatorname{garit}\left(B^{\bullet}\right) . A^{\bullet} \equiv \operatorname{gari}\left(A^{\bullet}, B^{\bullet}\right) & \forall A^{\bullet} \in \mathrm{GARI}_{\text {intern }}, \forall B^{\bullet} \in \mathrm{GARI} .
\end{array}
$$

Pay attention to the order of the terms, and observe that any bimould, acting on an internal, produces an internal

$$
\begin{aligned}
\operatorname{arit}(\mathrm{ARI}) . \mathrm{ARI}_{\text {intern }} & \subset \mathrm{ARI}_{\text {intern }}, \\
\operatorname{garit}(\mathrm{GARI}) . \mathrm{GARI}_{\text {intern }} & \subset \mathrm{GARI}_{\text {intern }} .
\end{aligned}
$$

\subsection{Short Guide to the Nomenclature}

Elements of Flex $(\mathfrak{E})$ or Flex $(\mathfrak{D})$ are always denoted by a short letter combination in Gothic fonts, with $\mathfrak{e}$ or $\mathfrak{o}$ as root vowels. The exchange $\mathfrak{e} \leftrightarrow \mathfrak{o}$ reflects the involution syap $^{17}$ while vowel change plus the Umlaut double $\operatorname{dot}(\mathfrak{e} \rightarrow \ddot{\mathfrak{o}}$ or $\mathfrak{o} \rightarrow \ddot{\mathfrak{e}})$ is expressive of the involution swap. $^{18}$

In the polar specializations, for reasons we cannot go into here, the conventions have to be slightly different: the root vowel here is $a$ (resp. $i$ ) for elements of Flex $(P a)$ (resp. Flex $(P i)$ ) but the exchange $a \leftrightarrow i$ under conservation of the consonental skeleton usually reflects the swap transform: thus pal $l^{\bullet} \leftrightarrow$ pil $^{\bullet}$ and par $^{\bullet} \leftrightarrow$ pir $^{\bullet}$. To express the syap transform, on the other hand, we usually change the final consonant plus of course the root vowel: thus pal $l^{\bullet} \leftrightarrow$ pir $^{\bullet}$ and pil $^{\bullet} \leftrightarrow$ par $^{\bullet}$. Since swap and syap thankfully commute, this leads to no major inconsistencies.

Lastly, inversion under the group laws, whether in the "Gothic" or "Roman" context, is usually denoted by a prefix reminiscent of the law: ri for gari, ra for gira, mи for $m и$. The same applies for the dilators, which take the prefix $d i, d a, d u$ depending on the parent group.

\section{Polar Alternals: the Series $\left\{\mathfrak{e} \mathfrak{e}_{r}^{\bullet}\right\},\left\{\mathfrak{l e}_{r}^{\bullet}\right\}$, and $\left\{\mathfrak{h} \mathfrak{e}_{r}^{\bullet}\right\},\left\{\mathfrak{k} \mathfrak{e}_{2 r}^{\bullet}\right\}$}

We shall construct in Flex(E) two elementary and two semi-elementary series of alternals by giving in each case a direct description side by side with an inductive definition.

\footnotetext{
${ }^{17}$ Which is a rigorous isomorphism for all flexion operations.

${ }^{18}$ Which respects few operations, but with an all-important exception: when acting on regular (i.e., neginvariant) bialternals or bisymmetrals, swap commutes respectively with ari or gari.
} 


\subsection{The First Alternal Series $\left\{\mathfrak{r} \mathfrak{e}_{r}^{\bullet}\right\}$}

The inductive definition, which immediately implies alternality, reads

$$
\mathfrak{r} \mathfrak{e}_{\mathbf{1}}^{\bullet}:=\mathfrak{E}^{\bullet} \quad ; \quad \mathfrak{r} \mathfrak{e}_{\mathbf{r}}^{\bullet}:=\operatorname{arit}\left(\mathfrak{r} \mathfrak{e}_{r-1}^{\bullet}\right) \mathbf{E}^{\bullet} \quad(\forall r \geq 2) .
$$

To get a direct definition-description of $\mathfrak{r e}_{r}^{\bullet}$, we may proceed like this. For any sign sequence $\boldsymbol{\epsilon}=\left\{\epsilon_{1}, \ldots, \epsilon_{r-1}\right\}$, we define the decreasing sets $J_{i}(\boldsymbol{\epsilon})$ by setting $J_{1}(\boldsymbol{\epsilon}):=$ $[1,2, \ldots, r]$ and, for $1<i \leq r$, by taking $J_{i}(\boldsymbol{\epsilon})$ to be $J_{i-1}(\boldsymbol{\epsilon})$ deprived of its largest (resp. smallest) element if $\epsilon_{i-1}=+$ (resp -). Then

$$
\mathfrak{r e}_{r}^{w_{1}, \ldots, w_{r}}:=\sum_{\epsilon_{1}, ., \epsilon_{r-1} \in\{+,-\}} \epsilon_{1} \ldots \epsilon_{r-1} \prod_{i=1}^{i=r} \mathfrak{E}^{\left(\begin{array}{c}
u_{i}^{*}(\boldsymbol{\epsilon}) \\
u_{i}^{*}(\boldsymbol{\epsilon})
\end{array}\right)}
$$

with indices $u_{i}^{*}(\boldsymbol{\epsilon}), v_{i}^{*}(\boldsymbol{\epsilon})$ defined by the dual conditions

$$
\begin{aligned}
& u_{i}^{*}(\boldsymbol{\epsilon}):=\sum u_{j} \quad \text { with } j \text { running through } J_{i}(\boldsymbol{\epsilon}), \\
& v_{i}^{*}(\boldsymbol{\epsilon}):=v_{j^{\prime}}-v_{j^{\prime \prime}} \quad \text { with } j^{\prime} \in J_{i}(\boldsymbol{\epsilon})-J_{i+1}(\boldsymbol{\epsilon}), j^{\prime \prime} \in J_{i-1}(\boldsymbol{\epsilon})-J_{i}(\boldsymbol{\epsilon}) .
\end{aligned}
$$

Of course, for $i=1$, we must set $v_{j^{\prime \prime}}=0$.

Alternatively, one may say that, when projected onto the standard basis $\left\{e_{t}^{\bullet}\right\}$ of Flex $(\mathfrak{E})$, the alternal $\mathfrak{r} \mathfrak{e}_{r}^{\bullet}$ takes the coefficient $(-1)^{k}$ when $\boldsymbol{t}$ is a one-branch tree with $k$ right-leaning slopes, and the coefficient 0 whenever $t$ has more than one branch.

The most outstanding property of the alternals $\mathfrak{r} \mathbf{e}_{r}^{\bullet}$ is their self-reproduction à la Witt under the ari bracket

$$
\operatorname{ari}\left(\mathfrak{r} \mathfrak{e}_{r_{1}}^{\bullet}, \mathfrak{r} \mathfrak{e}_{r_{2}}^{\bullet}\right)=\left(r_{1}-r_{2}\right) \mathfrak{r} \mathfrak{e}_{r_{1}+r_{2}}^{\bullet}
$$

\subsection{The Second Alternal Series $\left\{\mathfrak{l} \mathfrak{e}_{r}^{\bullet}\right\}$}

Here, the direct definition reads

$$
\mathfrak{l e}_{r}^{w_{1}, \ldots, w_{r}}:=\sum_{1 \leq i \leq r}(-1)^{i-1} \frac{(r-1) !}{(i-1) !(r-i) !} \mathfrak{E}^{\left(\begin{array}{c}
u_{1}+\ldots+u_{r} \\
v_{i}
\end{array}\right)} \prod_{j \neq i} \mathfrak{E}^{\left(\begin{array}{c}
u_{j} \\
v_{j}-v_{i}
\end{array}\right)} .
$$

Alternality is nearly obvious on this definition. It is even more obvious for the closely related bimoulds $\mathfrak{l e n}_{r}^{\bullet}$

$$
\mathfrak{l e n}_{r}^{w_{1}, \ldots, w_{r}}:=\sum_{1 \leq i \leq r}(-1)^{i-1} \frac{(r-1) !}{(i-1) !(r-i) !} \mathrm{I}^{\left(\begin{array}{c}
u_{i} \\
v_{i}
\end{array}\right)} \prod_{j \neq i} \mathfrak{E}^{\left(\begin{array}{c}
u_{j} \\
v_{j}
\end{array}\right)} .
$$

Clearly, $\mathfrak{l e n}_{r}^{\bullet}=$ duur.l $\mathfrak{e}_{r}^{\bullet}$, since we have on one hand

$$
\mathfrak{l}_{r}^{\bullet}=\sum_{1 \leq i \leq r}(-1)^{i-1} \frac{(r-1) !}{(i-1) !(r-i) !} \operatorname{amnit}\left(\operatorname{mu}_{\mathrm{i}-1}\left(\mathfrak{E}^{\bullet}\right), \operatorname{mu}_{\mathrm{r}-\mathrm{i}}\left(\mathfrak{E}^{\bullet}\right)\right) \cdot \mathfrak{E}^{\bullet}
$$

and on the other hand

$$
\mathfrak{l e n}_{r}^{\bullet}=\sum_{1 \leq i \leq r}(-1)^{i-1} \frac{(r-1) !}{(i-1) !(r-i) !} \operatorname{mu}\left(\operatorname{mu}_{\mathrm{i}-1}\left(\mathfrak{E}^{\bullet}\right), \mathrm{I}^{\bullet}, \operatorname{mu}_{\mathrm{r}-\mathrm{i}}\left(\mathfrak{E}^{\bullet}\right)\right)
$$

which again implies

$$
\mathfrak{l} \mathfrak{n}_{r}^{\bullet}=\operatorname{lu}(\mathrm{I}^{\bullet}, \overbrace{\mathfrak{E}^{\bullet}, \ldots, \mathfrak{E}^{\bullet}}^{(r-1)}) \text { times }
$$


This last expression (69) ensures the alternality of $\mathfrak{l e n}_{r}^{\bullet}$ and the earlier identity $\mathfrak{l} \mathfrak{n}_{r}^{\bullet}=$ duur. $\mathfrak{l e}_{r}^{\bullet}$ carries alternality back to $\mathfrak{l} \mathfrak{e}_{r}^{\bullet}$.

\subsection{The Third Alternal Series $\left\{\mathfrak{h} \mathfrak{e}_{r}^{\bullet}\right\}$}

We begin here with the direct, descriptive definition, which relies on the standard basis $\left\{e_{t}^{\bullet}\right\}$ of Flex $(\mathfrak{E})$. The coefficients $h e(t)$ of $\mathfrak{h} \mathfrak{e}_{r}^{\bullet}$ in that basis are not going to depend on the full structure of the indexing binary trees $\boldsymbol{t}$ but only on a four-parameter "abstract," $\operatorname{slant}(\boldsymbol{t})$, which gives the numbers $p_{1}, p_{2}$ (resp. $q_{1}, q_{2}$ ) of left-leaning (resp. right-leaning) slopes in the two branches issueing from the tree's root node. Clearly, $p_{1}+p_{2}+q_{1}+q_{2}=$ $r-1$, and the inductive calculation of $\operatorname{slant}(\boldsymbol{t})$ goes like this. If $\mathfrak{e}_{t}^{\bullet}=\operatorname{amnit}\left(\mathfrak{e}_{t^{\prime}}^{\bullet}, \mathfrak{e}_{t^{\prime \prime}}^{\bullet}\right) . \mathfrak{E}^{\bullet}$ with $\operatorname{slant}\left(\boldsymbol{t}^{\prime}\right)=\left[\begin{array}{l|l}p_{1}^{\prime} & p_{2}^{\prime} \\ q_{1}^{\prime} & q_{2}^{\prime}\end{array}\right]$ and $\operatorname{slant}\left(\boldsymbol{t}^{\prime \prime}\right)=\left[\begin{array}{l|l|l}p_{1}^{\prime \prime} & p_{2}^{\prime \prime} \\ q_{1}^{\prime \prime} & q_{2}^{\prime \prime}\end{array}\right]$, then

$$
\begin{aligned}
& \operatorname{slant}(\boldsymbol{t})=\left[\begin{array}{r|r}
1+p_{1}^{\prime}+p_{2}^{\prime} & p_{1}^{\prime \prime}+p_{2}^{\prime \prime} \\
q_{1}^{\prime}+q_{2}^{\prime} & 1+q_{1}^{\prime \prime}+q_{2}^{\prime \prime}
\end{array}\right] \quad \text { if } \quad \boldsymbol{t}^{\prime}, \boldsymbol{t}^{\prime \prime} \neq \emptyset, \\
& \operatorname{slant}(\boldsymbol{t})=\left[\begin{array}{r|r}
1+p_{1}^{\prime}+p_{2}^{\prime} & 0 \\
q_{1}^{\prime}+q_{2}^{\prime} & 0
\end{array}\right] \quad \text { if } \quad \boldsymbol{t}^{\prime \prime}=\emptyset, \\
& \operatorname{slant}(\boldsymbol{t})=\left[\begin{array}{r|r}
0 & p_{1}^{\prime \prime}+p_{2}^{\prime \prime} \\
0 & 1+q_{1}^{\prime \prime}+q_{2}^{\prime \prime}
\end{array}\right]
\end{aligned}
$$

We can now define $\mathfrak{e}_{t}^{\bullet}$

$$
\mathfrak{h} \mathfrak{e}_{r}^{\bullet}=\sum_{r(\bullet)=r} \operatorname{he}(\boldsymbol{t}) \mathfrak{e}_{t}^{\bullet}
$$

through coefficients $h e(t)=h e^{\left[\begin{array}{l|l}p_{1} & p_{2} \\ q_{1} & q_{2}\end{array}\right] \text { that depend only on } \operatorname{slant}(\boldsymbol{t})}$

$$
\text { he } \left.\begin{array}{l|l|l}
p_{1} & p_{2} \\
q_{1} & q_{2}
\end{array}\right]=(-1)^{q_{12}-1} \frac{\left(p_{12}\right) !\left(q_{12}\right) !}{\left(p_{12}+q_{12}\right) !} \operatorname{det}\left[\begin{array}{c|c}
p_{1} & 1+p_{2} \\
1+q_{1} & q_{2}
\end{array}\right]
$$

with the usual abbreviations $p_{12}:=p_{1}+p_{2}, q_{12}:=q_{1}+q_{2}$.

The invariance, implied by alternality, of the $\mathfrak{h} \mathfrak{e}^{\bullet}$ under

$$
\text { mantir }:=\text { minu.anti.pari }=- \text { anti.pari }
$$

is immediate since it amounts to

$$
\text { he }\left[\begin{array}{l|l}
p_{1} & p_{2} \\
q_{1} & q_{2}
\end{array}\right] \equiv(-1)^{p_{1}+p_{2}+q_{1}+q_{2}} \text { he }\left[\begin{array}{l|l|l}
q_{2} & q_{1} \\
p_{2} & p_{1}
\end{array}\right]
$$

but the full alternality is less obvious. It may be derived from the following identities. Indeed, setting

$$
\mathfrak{H} \mathfrak{e}^{\bullet}:=\sum_{1 \leq r} \frac{1}{r(r+1)} \mathfrak{h} \mathfrak{e}_{r}^{\bullet} ; \quad \mathfrak{R} \ddot{\mathfrak{e}} \bullet:=\sum_{1 \leq r} \frac{1}{r(\boldsymbol{r}+\mathbf{1})} \ddot{\mathfrak{r e}}_{r}^{\bullet}
$$

with $\mathfrak{r e}_{r}^{\bullet}:=\operatorname{swap} . \mathbf{r o}_{r}^{\bullet}$ for $\mathfrak{r o}_{r}^{\bullet}:=\operatorname{syap} . \mathfrak{r} \mathfrak{e}_{r}^{\bullet},{ }^{19}$ and introducing two elementary, mutually gani-inverse bimoulds $\mathfrak{s e}^{\bullet}, \mathfrak{n i s e}^{\bullet}$

$$
\begin{aligned}
& \mathfrak{s e}^{w_{1}}, \ldots, w_{r}:=\mathfrak{E}^{w_{1}} \ldots \mathfrak{E}^{w_{r}} \quad\left(\mathfrak{s e}^{\emptyset}:=1\right),
\end{aligned}
$$

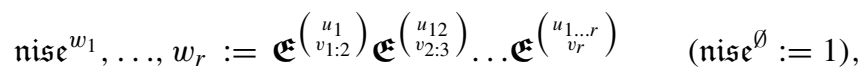

\footnotetext{
${ }^{19} \mathfrak{r} \mathfrak{o}_{r}^{\bullet}:=\operatorname{syap} \cdot \mathfrak{e} \mathfrak{e}_{r}^{\bullet}$ simply says that $\mathfrak{r} \mathfrak{o}_{r}^{\bullet}$ is constructed from $\mathfrak{O}$ exactly as $\mathfrak{r} \mathfrak{e}_{r}^{\bullet}$ was constructed from $\mathfrak{E}$.
} 
we can check (see (245)-(246)) either of the two equivalent identities

$$
\begin{aligned}
\mathfrak{H} \mathfrak{e}^{\bullet} & =\operatorname{ganit}\left(\mathfrak{n i s} \mathfrak{e}^{\bullet}\right) . \mathfrak{R} \dddot{\mathfrak{e}}^{\bullet}, \\
\mathfrak{R} \ddot{\mathfrak{e}} & =\operatorname{ganit}\left(\mathfrak{s} \mathfrak{e}^{\bullet}\right) \cdot \mathfrak{H} \mathfrak{e}^{\bullet} .
\end{aligned}
$$

Since $\mathfrak{R} \ddot{\mathfrak{e}}^{\bullet}$ is elementarily $\mathfrak{E}^{\bullet}$-alternal and since the mutually inverse operators $\operatorname{ganit}\left(\mathfrak{s e}^{\bullet}\right)$ and ganit $\left(\mathfrak{n i s e ^ { \bullet } )}\right.$ can be shown, almost as elementarily, to exchange $\mathfrak{E}^{\bullet}$-alternality and plain alternality

$$
\begin{aligned}
& \operatorname{ganit}\left(\mathfrak{s e}^{\bullet}\right): \text { alternal } \longrightarrow \mathfrak{E} \text { - alternal, }
\end{aligned}
$$

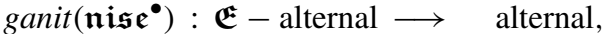

we conclude that $\mathfrak{H} \mathfrak{e}^{\bullet}$ is indeed alternal. The hard part in all this is to establish (79) or, preferably, (78). See the remarks in Section 4, towards the end of the second bisymmetrality proof. But if we do not want to bother with the messy combinatorics involved, we may simply take (78) as definition of $\mathfrak{H} \mathfrak{e}^{\bullet}$ and $\mathfrak{h} \mathfrak{e}_{r}^{\bullet}$. This route is calculation-free and automatically ensures the alternality of $\mathfrak{h} \mathfrak{e}_{r}^{\bullet}$.

\subsection{The Fourth Alternal Series $\left\{\mathfrak{k e}_{2 r_{*}}^{\bullet}\right\}$}

These new alternals are defined only for even lengths $r=2 r_{*}$. Like for the preceding series, we begin with a direct, descriptive definition by projection on the standard basis of Flex (E). Here too, the coefficients do not depend on the full structure of the indexing binary tree $t$ but on a four-parameter "abstract," stack $(\boldsymbol{t})$, which gives the numbers $m_{1}, m_{2}$ (resp. $n_{1}, n_{2}$ ) of end-nodes (resp. non end-nodes) carried by the two branches issueing from the root-node. Like in the previous case, we have $m_{1}+m_{2}+n_{1}+n_{2}=r-1$ but, unlike in the previous case, there now exist obvious inequalities between the $m_{i}$ 's and the $n_{i}$ 's. As a result, for any given (even) length $r$, the number of distinct stacks will be less than that of distinct slants.

The inductive definition of $\operatorname{stack}(\boldsymbol{t})$ goes like this. If $\mathfrak{e}_{t}^{\bullet}=\operatorname{amnit}\left(\mathfrak{e}_{t^{\prime}}^{\bullet}, \mathfrak{e}_{t^{\prime \prime}}^{\bullet}\right)$. $\mathfrak{E}^{\bullet}$ with

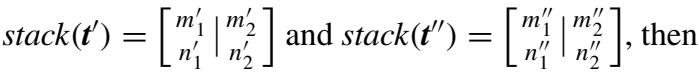

$$
\begin{aligned}
& \operatorname{stack}(\boldsymbol{t})=\left[\begin{array}{r|r}
m_{1}^{\prime}+m_{2}^{\prime} & p_{1}^{\prime \prime}+p_{2}^{\prime \prime} \\
1+n_{1}^{\prime}+n_{2}^{\prime} & 1+q_{1}^{\prime \prime}+q_{2}^{\prime \prime}
\end{array}\right] \quad \text { if } \quad \boldsymbol{t}^{\prime}, \boldsymbol{t}^{\prime \prime} \neq \emptyset, \\
& \operatorname{stack}(\boldsymbol{t})=\left[\begin{array}{c|c}
m_{1}^{\prime}+m_{2}^{\prime} & 0 \\
1+n_{1}^{\prime}+n_{2}^{\prime} & 0
\end{array}\right] \quad \text { if } \boldsymbol{t}^{\prime \prime}=\emptyset \text {, } \\
& \operatorname{stack}(\boldsymbol{t})=\left[\begin{array}{r|r}
0 & m_{1}^{\prime \prime}+m_{2}^{\prime \prime} \\
0 & 1+n_{1}^{\prime \prime}+n_{2}^{\prime \prime}
\end{array}\right] \quad \text { if } \quad \boldsymbol{t}^{\prime}=\emptyset \text {. }
\end{aligned}
$$

We are now in a position to define $\mathfrak{k}_{2}^{\bullet}{ }_{r_{*}}$

$$
\mathfrak{k} \mathfrak{e}_{2 r_{*}}^{\bullet}=\sum_{r(\boldsymbol{t})=2 r_{*}(\text { even })} \operatorname{ke}(\boldsymbol{t}) \mathfrak{e}_{t}^{\bullet}
$$

through coefficients $k e(\boldsymbol{t})=k e^{\left[\begin{array}{l|l}m_{1} & m_{2} \\ n_{1} & n_{2}\end{array}\right]}$ that depend only on $\operatorname{stack}(\boldsymbol{t})$

$$
\mathrm{ke}^{\left[\begin{array}{l|l}
m_{1} & m_{2} \\
n_{1} & n_{2}
\end{array}\right]}=(-2)^{m_{12}-1}\left(m_{12}-1\right) ! \frac{\left(n_{12}-m_{12}\right) ! !}{\left(n_{12}+m_{12}-2\right) ! !} \operatorname{det}\left[\begin{array}{c|c}
m_{1} & m_{2} \\
1+n_{1} & 1+n_{2}
\end{array}\right]
$$


with the usual abbreviations $m_{12}:=m_{1}+m_{2}, n_{12}:=n_{1}+n_{2}$ and with the odd or double factorial $^{20}$

$$
n ! !:=1.3 .5 \ldots(n-2) \cdot n=\frac{(n+1) !}{((n+1) / 2) !} 2^{-(n+1) / 2} \quad(\forall n \text { odd }) .
$$

The above definition of $\mathfrak{k e}_{2 r_{*}}^{\bullet}$ is concise enough, and striking too, but one thing it leaves in the dark ${ }^{21}$ is the alternality of $\mathfrak{k}_{2 r_{*}}^{\bullet}$. One way (and as far as we know, the only way) round this difficulty is to relate $\left\{\mathfrak{k} \mathfrak{e}_{2 r_{*}}^{\bullet}\right\}$ to $\left\{\mathfrak{h} \mathfrak{e}_{r}^{\bullet}\right\}$. To this end, we set

$$
\begin{aligned}
& \mathfrak{H} \mathfrak{e}^{\bullet}:=\sum_{1 \leq r} \frac{1}{r(r+1)} \mathfrak{h} \mathfrak{e}_{r}^{\bullet}, \\
& \mathfrak{H} \mathfrak{e}_{\mathrm{ev}}^{\bullet}:=\sum_{1 \leq r_{*}} \frac{1}{2 r_{*}\left(2 r_{*}+1\right)} \mathfrak{h} \mathfrak{e}_{2 r}^{\bullet}, \\
& \mathfrak{K} \mathfrak{e}^{\bullet}=\mathfrak{K} \mathfrak{e}_{\mathrm{ev}}^{\bullet}:=\sum_{1 \leq r_{*}} \frac{2^{-2 r_{*}+1}}{\left(2 r_{*}+1\right)\left(2 r_{*}-1\right)} \mathfrak{k} \mathfrak{e}_{2 r_{*}}^{\bullet}
\end{aligned}
$$

and we introduce the elementary operator $\mathcal{P}$ (adjoint action on $A R I$ )

$$
\mathcal{P} . M^{\bullet}:=\frac{1}{2} \operatorname{ari}\left(\mathfrak{E}^{\bullet}, \mathrm{M}^{\bullet}\right) \text {. }
$$

The thing is now to establish the identity

$$
\mathfrak{K} \mathfrak{e}_{\mathrm{ev}}^{\bullet}:=-\frac{1}{2} \mathfrak{E}^{\bullet}+\exp (\mathcal{P}) \cdot \mathfrak{H} \mathfrak{e}^{\bullet}
$$

or the equivalent but computationally more economical identity, which involves half as many terms

$$
\mathfrak{K} \mathfrak{e}_{\mathrm{ev}}^{\bullet}:=\cosh (\mathcal{P})^{-1} \cdot \mathfrak{H} \mathfrak{e}_{\mathrm{ev}}^{\bullet}
$$

and may be derived by inverting (90) to

$$
\mathfrak{H} \mathfrak{e}^{\bullet}:=\exp (-\mathcal{P}) \cdot\left(\frac{1}{2} \mathfrak{E}^{\bullet}+\mathfrak{K} \mathfrak{e}_{\mathrm{ev}}^{\bullet}\right) \equiv \exp (-\mathcal{P}) . \mathfrak{K} \mathfrak{e}_{\mathrm{ev}}^{\bullet},
$$

then parifying (92) to

$$
\mathfrak{H} \mathfrak{e}_{\mathrm{ev}}^{\bullet}:=\cosh (\mathcal{P}) . \mathfrak{K} \mathfrak{e}_{\mathrm{ev}}^{\bullet}
$$

and lastly inverting (93) back to (91).

For ways of establishing (90), we refer to the paragraph "properties of ripal ${ }_{e v}^{\bullet}$ " (see Section 4.7 below). But here again, if we are loath to go through the tedium of establishing (90) or (91) straight from the beautiful descriptive definition (83), we may forgo that direct definition and simply take (91) as the definition of $\mathfrak{k}_{2} r_{*}$. This is sufficient for all practical purposes and it gives us the alternality of $\mathfrak{k}_{2} r_{*}$ without our having to fire a single shot.

\footnotetext{
${ }^{20}$ This makes sense since the terms in the double factorials, namely $n_{12}+m_{12}-2$ and $n_{12}-m_{12}$, are always odd. The term $m_{12}-1$ may be even or odd, but that is no problem, as it sits in a simple factorial.

${ }^{21}$ Apart of course from the obvious relation anti $\mathfrak{k e r}_{2 r_{*}}^{\bullet} \equiv-\mathfrak{k e r}_{2 r_{*}}^{\bullet}$, which is necessary but far from sufficient for alternality.
} 
Remark: Parity Separation in $\left\{\mathfrak{h} \mathfrak{e}_{\boldsymbol{r}}^{\bullet}\right\}$ From (90) and (91), we derive, after elimination of $\mathfrak{K} \mathfrak{e}_{\mathrm{ev}}^{\bullet}$, an interesting way of expressing the odd-length components $\mathfrak{h} \mathfrak{e}_{2 r_{*}+1}^{\bullet}$ in terms of the even-length components. Indeed, setting

$$
\mathfrak{H} \mathfrak{e}^{\bullet}=\mathfrak{H} \mathfrak{e}_{\mathrm{ev}}^{\bullet}+\mathfrak{H} \mathfrak{e}_{\text {od }}^{\bullet}=\sum_{r \text { even }} \frac{1}{r(r+1)} \mathfrak{h} \mathfrak{e}_{r}^{\bullet}+\sum_{r \text { odd }} \frac{1}{r(r+1)} \mathfrak{h} \mathfrak{e}_{r}^{\bullet}
$$

we get

$$
\mathfrak{H} \mathfrak{e}_{\mathrm{od}}^{\bullet}=\frac{1}{2} \mathfrak{E}^{\bullet}+\tanh (\mathcal{P}) \cdot \mathfrak{H} \mathfrak{e}_{\mathrm{ev}}^{\bullet}
$$

Of course, $\exp (\mathcal{P}), \cosh (\mathcal{P}), \tanh (\mathcal{P})$, etc. should be interpreted as power series of the operator $\mathcal{P}$.

\subsection{Tables for Length $r=4$ : the Elementary Alternals}

$$
\begin{aligned}
& \text { basis element } \\
& \left|\mathfrak{r} \mathfrak{e}_{4}^{w}\right| \mathfrak{l} \mathfrak{e}_{4}^{w} \mid \\
& \mathfrak{e}_{[1,2,3,4]}^{w_{1}, w_{2}, w_{3}, w_{4}}=\mathfrak{E}^{\left(\begin{array}{c}
u_{1234} \\
v_{4}
\end{array}\right)} \mathfrak{E}^{\left(\begin{array}{c}
u_{123} \\
v_{3}: 4
\end{array}\right)} \mathfrak{E}^{\left(\begin{array}{c}
u_{12} \\
v_{2: 3}
\end{array}\right)} \mathfrak{E}^{\left(\begin{array}{c}
u_{1} \\
v_{1: 2}
\end{array}\right)}|1|-1 \mid
\end{aligned}
$$

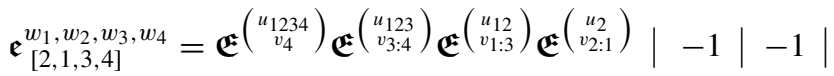

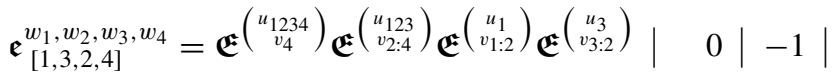

$$
\begin{aligned}
& \mathfrak{e}_{[2,3,1,4]}^{w_{1}, w_{2}, w_{3}, w_{4}}=\mathfrak{E}^{\left(\begin{array}{c}
u_{1234} \\
v_{4}
\end{array}\right)} \mathfrak{E}^{\left(\begin{array}{c}
u_{123} \\
v_{1: 4}
\end{array}\right)} \mathfrak{E}^{\left(\begin{array}{c}
u_{23} \\
v_{3: 1}
\end{array}\right)} \mathfrak{E}^{\left(\begin{array}{c}
u_{2} \\
v_{2: 3}
\end{array}\right)}|-1|-1 \mid \\
& \mathfrak{e}_{[3,2,1,4]}^{w_{1}, w_{2}, w_{3}, w_{4}}=\mathfrak{E}^{\left(\begin{array}{c}
u_{1234} \\
v_{4}
\end{array}\right)} \mathfrak{E}^{\left(\begin{array}{c}
u_{123} \\
v_{1: 4}
\end{array}\right)} \mathfrak{E}^{\left(\begin{array}{c}
u_{23} \\
v_{2: 1}
\end{array}\right)} \mathfrak{E}^{\left(\begin{array}{c}
u_{3} \\
v_{3: 2}
\end{array}\right)}|1|-1 \mid \\
& \mathfrak{e}_{[1,2,4,3]}^{w_{1}, w_{2}, w_{3}, w_{4}}=\mathfrak{E}^{\left(\begin{array}{c}
u_{1234} \\
v_{3}
\end{array}\right)} \mathfrak{E}^{\left(\begin{array}{c}
u_{12} \\
v_{2: 3}
\end{array}\right)} \mathfrak{E}^{\left(\begin{array}{c}
u_{1} \\
v_{1: 2}
\end{array}\right)} \mathfrak{E}^{\left(\begin{array}{c}
u_{4} \\
v_{4: 3}
\end{array}\right)}|0| 3 \mid \\
& \mathfrak{e}_{[2,1,4,3]}^{w_{1}, w_{2}, w_{3}, w_{4}}=\mathfrak{E}^{\left(\begin{array}{c}
u_{1234} \\
v_{3}
\end{array}\right)} \mathfrak{E}^{\left(\begin{array}{c}
u_{12} \\
v_{1: 3}
\end{array}\right)} \mathfrak{E}^{\left(\begin{array}{c}
u_{2} \\
v_{2: 1}
\end{array}\right)} \mathfrak{E}^{\left(\begin{array}{c}
u_{4} \\
v_{4}
\end{array}\right)}|\quad 0| 3 \mid \\
& \mathfrak{e}_{[1,3,4,2]}^{w_{1}, w_{2}, w_{3}, w_{4}}=\mathfrak{E}^{\left(\begin{array}{c}
u_{1234} \\
v_{2}
\end{array}\right)} \mathfrak{E}^{\left(\begin{array}{c}
u_{1} \\
v_{1: 2}
\end{array}\right)} \mathfrak{E}^{\left(\begin{array}{c}
u_{34} \\
v_{4: 2}
\end{array}\right)} \mathfrak{E}^{\left(\begin{array}{c}
u_{3} \\
v_{3: 4}
\end{array}\right)}|0|-3 \mid
\end{aligned}
$$

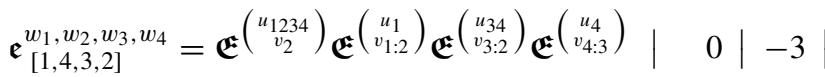

$$
\begin{aligned}
& \mathfrak{e}_{[2,3,4,1]}^{w_{1}, w_{2}, w_{3}, w_{4}}=\mathfrak{E}^{\left(\begin{array}{c}
u_{1234} \\
v_{1}
\end{array}\right)} \mathfrak{E}^{\left(\begin{array}{c}
u_{234} \\
v_{4: 1}
\end{array}\right)} \mathfrak{E}^{\left(\begin{array}{c}
u_{23} \\
v_{3: 4}
\end{array}\right)} \mathfrak{E}^{\left(\begin{array}{c}
u_{2} \\
v_{2: 3}
\end{array}\right)}|-1| 1 \mid \\
& \mathfrak{e}_{[3,2,4,1]}^{w_{1}, w_{2}, w_{3}, w_{4}}=\mathfrak{E}^{\left(\begin{array}{c}
u_{1234} \\
v_{1}
\end{array}\right)} \mathfrak{E}^{\left(\begin{array}{c}
u_{234} \\
v_{4: 1}
\end{array}\right)} \mathfrak{E}^{\left(\begin{array}{c}
u_{23} \\
v_{2: 4}
\end{array}\right)} \mathfrak{E}^{\left(\begin{array}{c}
u_{3} \\
v_{3: 2}
\end{array}\right)}|\quad 1| \quad 1 \mid \\
& \mathfrak{e}_{[2,4,3,1]}^{w_{1}, w_{2}, w_{3}, w_{4}}=\mathfrak{E}^{\left(\begin{array}{c}
u_{1234} \\
v_{1}
\end{array}\right)} \mathfrak{E}^{\left(\begin{array}{c}
u_{234} \\
v_{3: 1}
\end{array}\right)} \mathfrak{E}^{\left(\begin{array}{c}
u_{2} \\
v_{2: 3}
\end{array}\right)} \mathfrak{E}^{\left(\begin{array}{c}
u_{4} \\
v_{4: 3}
\end{array}\right)}|\quad 0| \quad 1 \mid \\
& \mathfrak{e}_{[3,4,2,1]}^{w_{1}, w_{2}, w_{3}, w_{4}}=\mathfrak{E}^{\left(\begin{array}{c}
u_{1234} \\
v_{1}
\end{array}\right)} \mathfrak{E}^{\left(\begin{array}{c}
u_{234} \\
v_{2}: 1
\end{array}\right)} \mathfrak{E}^{\left(\begin{array}{c}
u_{34} \\
v_{4: 2}
\end{array}\right)} \mathfrak{E}^{\left(\begin{array}{c}
u_{3} \\
v_{3: 4}
\end{array}\right)}|\quad 1| \quad 1 \mid \\
& \mathfrak{e}_{[4,3,2,1]}^{w_{1}, w_{2}, w_{3}, w_{4}}=\mathfrak{E}^{\left(\begin{array}{c}
u_{1234} \\
v_{1}
\end{array}\right)} \mathfrak{E}^{\left(\begin{array}{c}
u_{234} \\
v_{2: 1}
\end{array}\right)} \mathfrak{E}^{\left(\begin{array}{c}
u_{34} \\
v_{3: 2}
\end{array}\right)} \mathfrak{E}^{\left(\begin{array}{c}
u_{4} \\
v_{4: 3}
\end{array}\right)}|-1| 1 \mid
\end{aligned}
$$


Tables for Length $r=4$ : the Semi-elementary Alternals.

$$
\begin{aligned}
& \text { basis element } \\
& \text { slant }\left|\mathfrak{h} \mathfrak{e}_{4}^{w}\right| \operatorname{stack}\left|\mathfrak{k} \mathfrak{e}_{4}^{w}\right|
\end{aligned}
$$

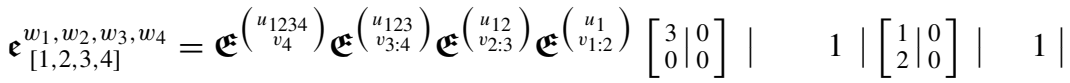

$$
\begin{aligned}
& \mathfrak{e}_{[2,1,3,4]}^{w_{1}, w_{2}, w_{3}, w_{4}}=\mathfrak{E}^{\left(\begin{array}{c}
u_{1234} \\
v_{4}
\end{array}\right)} \mathfrak{E}^{\left(\begin{array}{c}
u_{123} \\
v_{3: 4}
\end{array}\right)} \mathfrak{E}^{\left(\begin{array}{c}
u_{12} \\
v_{1: 3}
\end{array}\right)} \mathfrak{E}^{\left(\begin{array}{c}
u_{2} \\
v_{2: 1}
\end{array}\right)}\left[\begin{array}{l}
2 \\
1
\end{array} \mid \begin{array}{l}
0 \\
0
\end{array}\right]|-2 / 3|\left[\begin{array}{l}
1 \\
2 \\
0
\end{array}\right]|1| \\
& \mathfrak{e}_{[1,3,2,4]}^{w_{1}, w_{2}, w_{3}, w_{4}}=\mathfrak{E}^{\left(\begin{array}{c}
u_{1234} \\
v_{4}
\end{array}\right)} \mathfrak{E}^{\left(\begin{array}{c}
u_{123} \\
v_{2: 4}
\end{array}\right)} \mathfrak{E}^{\left(\begin{array}{c}
u_{1} \\
v_{1: 2}
\end{array}\right)} \mathfrak{E}^{\left(\begin{array}{c}
u_{3} \\
v_{3: 2}
\end{array}\right)}\left[\begin{array}{l}
2 \\
1
\end{array} \mid \begin{array}{l}
0 \\
0
\end{array}\right]|-2 / 3|\left[\begin{array}{l}
2 \\
1
\end{array} \mid \begin{array}{l}
0 \\
0
\end{array}\right]|-4| \\
& \mathfrak{e}_{[2,3,1,4]}^{w_{1}, w_{2}, w_{3}, w_{4}}=\mathfrak{E}^{\left(\begin{array}{c}
u_{1234} \\
v_{4}
\end{array}\right)} \mathfrak{E}^{\left(\begin{array}{c}
u_{123} \\
v_{1: 4}
\end{array}\right)} \mathfrak{E}^{\left(\begin{array}{c}
u_{23} \\
v_{3: 1}
\end{array}\right)} \mathfrak{E}^{\left(\begin{array}{c}
u_{2} \\
v_{2: 3}
\end{array}\right)}\left[\begin{array}{l}
2 \\
1
\end{array} \mid \begin{array}{l}
0 \\
0
\end{array}\right]|-2 / 3|\left[\begin{array}{l}
1 \\
2
\end{array} \mid \begin{array}{l}
0 \\
0
\end{array}\right]|1|
\end{aligned}
$$

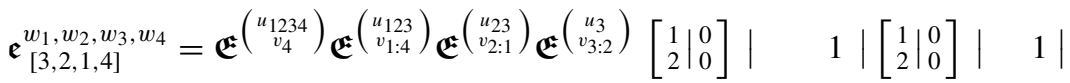

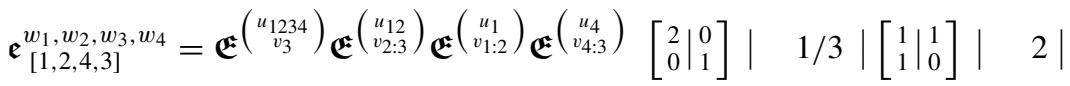

$$
\begin{aligned}
& \mathfrak{e}_{[2,1,4,3]}^{w_{1}, w_{2}, w_{3}, w_{4}}=\mathfrak{E}^{\left(\begin{array}{c}
u_{1234} \\
v_{3}
\end{array}\right)} \mathfrak{E}^{\left(\begin{array}{c}
u_{12} \\
v_{1: 3}
\end{array}\right)} \mathfrak{E}^{\left(\begin{array}{c}
u_{2} \\
v_{2: 1}
\end{array}\right)} \mathfrak{E}^{\left(\begin{array}{c}
u_{4} \\
v_{4: 3}
\end{array}\right)}\left[\begin{array}{l}
1 \\
1
\end{array} \mid \begin{array}{l}
0 \\
1
\end{array}\right]|\quad 1 / 3|\left[\begin{array}{l}
1 \\
1
\end{array} \mid \begin{array}{l}
1 \\
0
\end{array}\right]|2|
\end{aligned}
$$

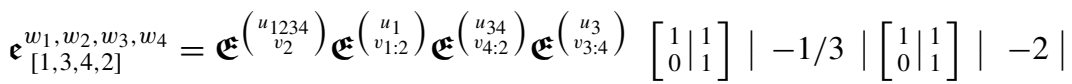

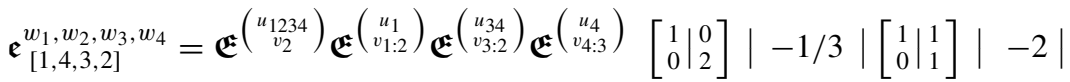

$$
\begin{aligned}
& \mathfrak{e}_{[2,3,4,1]}^{w_{1}, w_{2}, w_{3}, w_{4}}=\mathfrak{E}^{\left(\begin{array}{c}
u_{1234} \\
v_{1}
\end{array}\right)} \mathfrak{E}^{\left(\begin{array}{c}
u_{234} \\
v_{4: 1}
\end{array}\right)} \mathfrak{E}^{\left(\begin{array}{c}
u_{23} \\
v_{3: 4}
\end{array}\right)} \mathfrak{E}^{\left(\begin{array}{c}
u_{2} \\
v_{2: 3}
\end{array}\right)}\left[\begin{array}{l}
0 \\
0
\end{array}\right]|-1|\left[\begin{array}{l}
0 \\
0
\end{array}\right]|-1|
\end{aligned}
$$

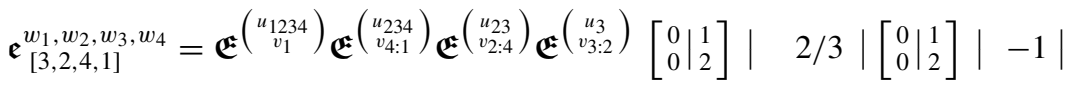

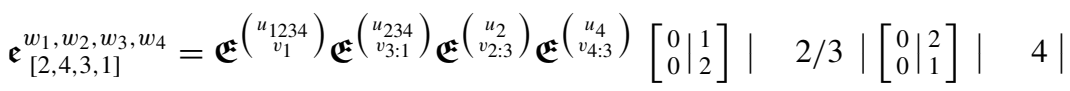

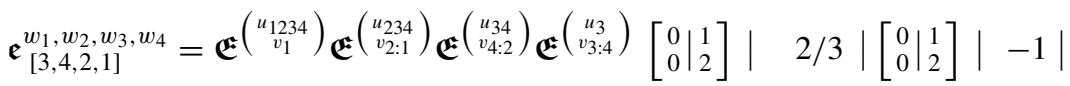

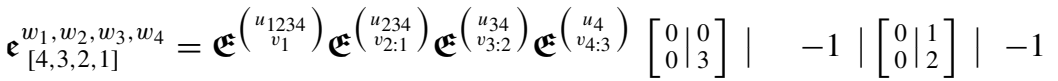

\section{Polar Bisymmetrals: Main Statements}

For perspective, let us start with a synoptic table of our central bimoulds:

$$
\begin{aligned}
& \mathfrak{e s s}^{\bullet} \stackrel{\text { swap }}{\leftrightarrow} \ddot{\mathfrak{o}} \mathfrak{s s}^{\bullet} \quad(\mathfrak{E} \mapsto \mathrm{Pi}) \quad \text { pil } \stackrel{\text { swap }}{\leftrightarrow} \quad \mathrm{pal}^{\bullet}
\end{aligned}
$$

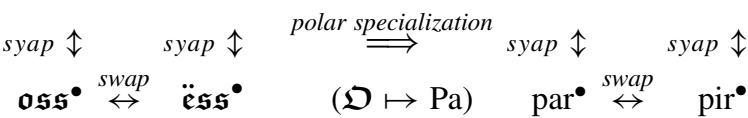

We take our stand on the self-reproduction property (66) of the alternals $\mathfrak{r} \mathfrak{e}_{r}^{\bullet}$ under the ari bracket, which is entirely analogous to the behavior of the monomials $x^{r+1}$ under the bracket $\{\phi, \psi\}:=\phi^{\prime} \psi-\phi \psi^{\prime}$. As a consequence, the Lie algebra isomorphism 
induced by $x^{r+1} \mapsto \mathfrak{r} \mathfrak{e}_{r}^{\bullet}$ extends to an isomorphism of the group of formal identitytangent mappings $f:=x \mapsto x+\sum a_{r} x^{r+1}$ into the group GARI $I_{r e}$ consisting of bimoulds of the form $S^{\bullet}:=\operatorname{expari}\left(\sum \gamma_{r} \mathfrak{r e}_{r}^{\bullet}\right)$. All elements of $G A R I_{r e}$ are automatically symmetral.

Proposition 3.1 (Direct bisymmetral: definition) The source mapping $f: x \mapsto 1-e^{-x}=$ $x-1 / 2 x^{2}+\ldots$ has for images in GARI $I_{\mathfrak{r}}$, resp. GARI $I_{\mathfrak{r} o}$, bimoulds denoted by $\mathfrak{e s s}^{\bullet}$, resp. $\mathfrak{o s s}^{\bullet}$. They are automatically symmetral, but their swappees $\ddot{\mathfrak{o} s s^{\bullet}}$, resp. $\ddot{\mathfrak{e s s}} \mathfrak{s}^{\bullet}$, are also symmetral. The same-vowelled bimoulds $\mathfrak{e s s}$ and $\ddot{\mathfrak{e}} \mathfrak{s s}$ (and by way of consequence $\mathfrak{o s s}$ and $\ddot{\mathfrak{o s s}})$ coincide up to length $r=3$ inclusively but differ ever after. Under the polar specialization $(\mathfrak{O}, \mathfrak{E}) \mapsto(P a, P i)$ our universal bimoulds specialize to

$$
\begin{aligned}
&\left(\ddot{\mathfrak{o s s}} \mathfrak{s}^{\bullet}, \mathfrak{e s s} \mathfrak{s}^{\bullet}\right) \mapsto\left(\mathrm{pal}^{\bullet}, \mathrm{pil}^{\bullet}\right), \\
&\left(\mathfrak{o} \mathfrak{s s}^{\bullet}, \ddot{\mathfrak{e}} \mathfrak{s s ^ { \bullet }}\right) \mapsto\left(\text { par }^{\bullet}, \text { pir }^{\bullet}\right) .
\end{aligned}
$$

At this point, the reader may well ask: why, among all identity-tangent mappings $f$, single out precisely $f: x \mapsto 1-e^{-x}$ ? The short answer is: because only this choice and no other ${ }^{22}$ ensures that the separator $\operatorname{gepar}\left(\mathfrak{e s s}^{\bullet}\right)$ is symmetral (see (109)) below), which in turn is a necessary condition for $\ddot{\mathfrak{o}} \mathfrak{s s}^{\bullet}$ (not $\mathfrak{e s s}^{\bullet}$ !) to be symmetral. The condition, however, is not sufficient, and the full bisymmetrality proofs (two of them), as indeed all the other proofs backing up this section's statements, will be given in Section 4.

Proposition 3.2 (Direct bisymmetral: characterization) The bimould pal has only poles of the form $P\left(u_{i}\right)$ or $P\left(u_{1}+\ldots+u_{2 i}\right)$. Equivalently, its swappee pil ${ }^{\bullet}$, or rather anti.pil ${ }^{\bullet}$, has only poles of the form ${ }^{23} P\left(v_{i}-v_{i-1}\right)$ or $P\left(v_{2 i}\right)$. This pole pattern characterizes pal ${ }^{\bullet} /$ pil $^{\bullet}$ among all other polar bisymmetrals.

Proposition 3.3 (Inverse bisymmetral: properties) The gari-inverses (prefix "ri") of the bisymmetrals are automatically symmetral, but they are not bisymmetral, meaning that their swappees, which may also be viewed as gira-inverses (prefix "ra") are not exactly symmetral, but rather $\mathfrak{E}$-symmetral or $\mathfrak{D}$-symmetral, depending of course on the root vowel. Thus side by side with the straight symmetries

$$
\begin{aligned}
& \operatorname{riess} \mathfrak{s}^{\bullet}=\operatorname{invgari}\left(\mathfrak{e s s}^{\bullet}\right) \text { and } \operatorname{riëss} \mathfrak{s}^{\bullet}=\operatorname{invgari}\left(\ddot{\mathfrak{e}} \mathfrak{s s}^{\bullet}\right) \in \text { symmetral, } \\
& \mathfrak{r i e s s}^{\bullet}=\operatorname{invgari}\left(\mathfrak{e s s}^{\bullet}\right) \text { and } \mathfrak{r i o ̈ s s}^{\bullet}=\operatorname{invgari}\left(\ddot{\mathfrak{o s s s}}{ }^{\bullet}\right) \in \text { symmetral }
\end{aligned}
$$

we have the tweaked symmetries

$$
\begin{aligned}
& \operatorname{raess} s^{\bullet}=\operatorname{invgira}\left(\mathfrak{e s s}^{\bullet}\right)=\operatorname{swap}\left(\mathfrak{r i o ̈ s s} s^{\bullet}\right) \in \mathfrak{E}-\text { symmetral }, \\
& \mathfrak{r a e s s s ^ { \bullet }}=\operatorname{invgira}\left(\ddot{\mathfrak{e} s s^{\bullet}}\right)=\operatorname{swap}\left(\operatorname{rioss}^{\bullet}\right) \in \mathfrak{E}-\text { symmetral } \text {, } \\
& \mathfrak{r a o s s ^ { \bullet }}=\operatorname{invgira}\left(\mathfrak{o s s}^{\bullet}\right)=\operatorname{swap}(\mathfrak{r i e ̈ s s}) \in \mathfrak{O}-\text { symmetral }, \\
& \mathfrak{r a} \ddot{\mathfrak{o}} \mathfrak{s s}^{\bullet}=\operatorname{invgira}\left(\ddot{\mathfrak{o}} \mathfrak{s s}^{\bullet}\right)=\operatorname{swap}\left(\boldsymbol{r i e s s} \mathfrak{s}^{\bullet}\right) \in \mathfrak{D}-\text { symmetral } .
\end{aligned}
$$

\footnotetext{
${ }^{22}$ That is, up to a rescaling $f \mapsto f_{c}$ with $f_{c}: x \mapsto c^{-1} f(c x)$. But the applications we have in mind, as well as intrinsic considerations, dictate that we take $c=1$.

${ }^{23}$ For $i=1$, " $P\left(v_{1}-v_{0}\right)$ " of course reduces to $P\left(v_{1}\right)$.
} 
In the polar specialization $(\mathfrak{O}, \mathfrak{E}) \mapsto(P a, P i)$, this becomes

$$
\begin{aligned}
& \text { ripal }^{\bullet}, \operatorname{ripar}^{\bullet}, \operatorname{ripil}^{\bullet}, \operatorname{ripir}^{\bullet}, \in \text { symmetral, } \\
& \text { rapil }^{\bullet}=\text { swap.ripal }^{\bullet}, \operatorname{rapir}^{\bullet}=\text { swap.ripar }{ }^{\bullet} \in \text { symmetril }, \\
& \text { rapal }^{\bullet}=\text { swap.ripil }^{\bullet}, \operatorname{rapar}^{\bullet}=\text { swap.ripir }{ }^{\bullet} \in \text { symmetrul } .
\end{aligned}
$$

We now recall the definition of the two separators ${ }^{24}$ gepar and hepar

$$
\begin{aligned}
& \text { gepar. } S^{\bullet}:=\operatorname{mu}\left(\text { anti.swap. } S^{\bullet} \text {, swap. } S^{\bullet}\right) \\
& \text { hepar. } S^{\bullet}:=\sum_{1 \leq k \leq r(\bullet)} \text { pus }^{\mathrm{k}} . \operatorname{logmu} . \text { swap. } S^{\bullet}
\end{aligned}
$$

Proposition 3.4 (Direct bisymmetral: separators) The separation identities read

$$
\begin{aligned}
& \text { gepar. } \mathfrak{e s s}^{\bullet}:=\quad m u\left(\operatorname{anti.} \ddot{\mathfrak{o}} \mathfrak{s s}^{\bullet}, \ddot{\mathfrak{o}} \mathfrak{s s}^{\bullet}\right)=\operatorname{expmu}\left(-\mathfrak{O}^{\bullet}\right), \\
& \text { hepar.ess } \mathfrak{s i}^{\bullet}=\sum_{1 \leq k \leq r(\bullet)} \text { pus }^{k} \cdot \operatorname{logmu} . \ddot{\mathbf{o s s}} \mathfrak{s}^{\bullet}=-\frac{1}{2} \mathfrak{O}^{\bullet}
\end{aligned}
$$

with their obvious analogues under the exchange $\mathfrak{e} \leftrightarrow \mathfrak{o}$.

Proposition 3.5 (Inverse bisymmetral: separators) The separation identities read

$$
\begin{aligned}
& \text { gepar.riess } \mathfrak{e s}^{\bullet}=\operatorname{mu}\left(\text { anti.räass } \mathfrak{s}^{\bullet}, \mathfrak{r a} \ddot{\mathfrak{o} s s^{\bullet}}\right)=1^{\bullet}+\sum_{r \geq 1} \operatorname{mu}_{r}\left(\mathfrak{O}^{\bullet}\right) \text {, } \\
& \text { hepar.riess }{ }^{\bullet}:=\sum_{1 \leq k \leq r(\bullet)} \operatorname{pus}^{k} \cdot \operatorname{logmu} \cdot \mathfrak{r a ̈ a ̈ s s} \mathfrak{s}^{\bullet}=\frac{1}{2} \sum_{r \geq 1} \operatorname{mu}_{r}\left(\mathfrak{D}^{\bullet}\right) \text {. }
\end{aligned}
$$

They possess obvious analogues under the exchange $\mathfrak{e} \leftrightarrow$ o. Here, $\operatorname{mu}_{r}\left(\mathfrak{O}^{\bullet}\right)$ stands, as usual, for the rth mu-power of $\mathfrak{O}$.

Proposition 3.6 (Direct bisymmetral: gari-dilator) The identity reads

$$
\begin{aligned}
\operatorname{der} . \mathfrak{s s}^{\bullet} & =\operatorname{preari}\left(\mathfrak{e s s}^{\bullet}, \mathfrak{d i e s s}^{\bullet}\right) \quad \text { with } \\
\mathfrak{d i e s s}^{\bullet} & :=-\sum_{r \geq 1} \frac{1}{(1+r) !} \mathfrak{r e} \mathbf{e}_{r}^{\bullet} \in \text { alternal }
\end{aligned}
$$

and has an obvious analogue under the exchange $\mathfrak{e} \leftrightarrow \mathfrak{o}$.

Proposition 3.7 (Inverse bisymmetral: gari-dilator) The identities read

$$
\begin{aligned}
& \text { der.riess }{ }^{\bullet}=\operatorname{preari}\left(\operatorname{riess}^{\bullet}, \operatorname{ditiess}^{\bullet}\right), \\
& \operatorname{der} . \mathfrak{x} \ddot{\mathfrak{o}} \mathfrak{s s}^{\bullet}=\operatorname{preari}\left(r i \ddot{o} \mathfrak{s s}^{\bullet}, \operatorname{diriöss}{ }^{\bullet}\right)
\end{aligned}
$$

\footnotetext{
${ }^{24}$ So-called because, acting on elements $S^{\bullet}$ of the group $G A R I_{\mathfrak{r} \mathfrak{e}}$, they have the virtue of separating (or manifesting, if you prefer) the coefficients $a_{r}$ of the source mapping $f$ : see the remarks immediately before Proposition 3.1 and also [4] Section 4.1.
} 
with dilators equal to

$$
\begin{aligned}
\mathfrak{d i r i e s s}^{\bullet} & :=+\sum_{r \geq 1} \frac{1}{r .(1+r)} \mathfrak{k} \mathfrak{e}_{r}^{\bullet} \in \text { alternal, } \\
\mathfrak{d i x i o ̈ s s s} & :=+\sum_{r \geq 1} \frac{1}{r .(1+r)} \mathfrak{h} \mathfrak{o}_{r}^{\bullet} \in \text { alternal }
\end{aligned}
$$

and with the semi-elementary alternals $\mathfrak{h o}_{r}^{\bullet}$ defined as in (73) but based on the unit $\mathfrak{O}$ instead of $\mathfrak{E}$.

Proposition 3.8 (Bisymmetral swappee: $m u$-dilator) The identity reads

$$
\begin{aligned}
& \ddot{\mathfrak{o} s s^{\bullet}}=\operatorname{muu}(\ddot{\mathfrak{o s s}}, \mathbf{d u u} \ddot{\mathfrak{o} s \mathfrak{s})} \text { with } \\
& \text { duuöss } \ddot{s s}^{\bullet}=+\sum_{r \geq 1} \alpha_{r} \mathfrak{l o}_{r}^{\bullet} \in \text { alternal }
\end{aligned}
$$

with muu defined as in (25) and the elementary alternals $\mathfrak{l o}_{r}^{\bullet}$ defined as in Section 2 but with respect to the unit $\mathfrak{D}$ instead of $\mathfrak{E}$. The coefficients $\alpha_{r}$ are the Bernoulli numbers

$$
\sum_{r \geq 1} \alpha_{r} t^{r}:=-1+\frac{t}{e^{t}-1}=-\frac{1}{2} t+\frac{1}{12} t^{2}-\frac{1}{720} t^{4}+\frac{1}{30240} t^{6}+\ldots
$$

Under the polar specialization $\mathfrak{O} \mapsto P a$, the above relations assume the simpler form

$$
\begin{aligned}
\text { dur.pal } & \left.=\text { mu.(pal }{ }^{\bullet}, \text { dupal }^{\bullet}\right), \\
\text { dupal }^{\bullet} & :=\sum_{r \geq 1} \alpha_{r} \operatorname{lan}_{r}^{\bullet}
\end{aligned}
$$

relatively to the elementary alternals

$$
\operatorname{lan}_{r}^{\bullet}:=\operatorname{lu}(\mathrm{I}^{\bullet}, \overbrace{P a^{\bullet}, \ldots, P a^{\bullet}}^{r-1 \text { times }}) .
$$

Before examining the parity properties of our bisymmetrals, a few general considerations are in order. It is clear that any bimould $M^{\bullet}$ such that $M^{\emptyset}=1$ can be uniquely factored as follows

$$
M^{\bullet}=\operatorname{gari}\left(M_{\mathrm{od}}^{\bullet}, M_{\mathrm{ev}}^{\bullet}\right)=\operatorname{mu}\left(M_{\mathrm{odd}}^{\bullet}, M_{\mathrm{evv}}^{\bullet}\right)
$$

or in reverse order

$$
M^{\bullet}=\operatorname{gari}\left(M_{\mathrm{ev}}^{\bullet}, M_{\mathrm{od}}^{\bullet}\right)=\operatorname{mu}\left(M_{\mathrm{evv}}^{\bullet}, M_{\mathrm{odd}}^{\bullet}\right)
$$

with factors that of course differ from (125) to (126) but in both cases satisfy the parity conditions

$$
\begin{aligned}
& \text { pari. } M_{\mathrm{ev}}^{\bullet} \equiv M_{\mathrm{ev}}^{\bullet} \quad ; \quad \text { pari. } M_{\mathrm{od}}^{\bullet} \equiv \text { invgari. } M_{\mathrm{od}}^{\bullet} \\
& \text { pari. } M_{\mathrm{evv}}^{\bullet} \equiv M_{\mathrm{evv}}^{\bullet} ; \quad \text { pari. } M_{\mathrm{odd}}^{\bullet} \equiv \text { invmu. } M_{\mathrm{odd}}^{\bullet}
\end{aligned}
$$

With the "upper" factorizations (125), for example, we find

$$
\begin{aligned}
\operatorname{gari}\left(M_{\mathrm{od}}^{\bullet}, M_{\mathrm{od}}^{\bullet}\right) & =\operatorname{gari}\left(M^{\bullet}, \text { pari.invgari. } M^{\bullet}\right), \\
\operatorname{mu}\left(M_{\mathrm{odd}}^{\bullet}, M_{\mathrm{odd}}^{\bullet}\right) & =\operatorname{mu}\left(M^{\bullet}, \text { pari.invmu. } M^{\bullet}\right) .
\end{aligned}
$$

From there, by square rooting, ${ }^{25}$ we go to $M_{\mathrm{od}}^{\bullet}$ and $M_{\mathrm{odd}}^{\bullet}$ and thence to $M_{\mathrm{ev}}^{\bullet}$ and $M_{\mathrm{evv}}^{\bullet}$.

\footnotetext{
${ }^{25}$ An unambiguous operation, if we impose, as we do, that $M^{\emptyset}=M_{\mathrm{od}}^{\emptyset}=M_{\mathrm{ev}}^{\emptyset}=M_{\mathrm{odd}}^{\emptyset}=M_{\mathrm{evv}}^{\emptyset}=1$.
} 
None of this requires $M^{\bullet}$ to be symmetral or in Flex $(\mathfrak{E})$. Elements of Flex(E), though, behave identically under pari and neg, so that for them the labels even and odd acquire redoubled significance.

In any case, the existence of even $\times$ odd or odd $\times$ even factorizations is a universal phenomenon. ${ }^{26}$ What distinguishes the bisymmetrals is the existence of remarkable and multiple factorizations of that sort, with odd factors that tend to be exceedingly simple.

Proposition 3.9 (Parity properties) We have three similar-looking but logically independent identities:

$$
\begin{aligned}
& \mathfrak{e s s}^{\bullet}=\operatorname{gari}\left(\mathfrak{e s s}_{\mathrm{od}}^{\bullet}, \mathfrak{e s s}_{\mathrm{ev}}^{\bullet}\right), \\
& \ddot{\mathfrak{o}} \mathfrak{s s}^{\bullet}=\operatorname{gari}\left(\ddot{\mathfrak{o}} \mathfrak{s s _ { \mathrm { d } } ^ { \bullet }}, \ddot{\mathfrak{o}} \mathfrak{s s}_{\mathrm{ev}}^{\bullet}\right) \text {, } \\
& \ddot{\mathfrak{o}} \mathfrak{s s} \mathfrak{s}^{\bullet}=\operatorname{mu}\left(\ddot{\mathfrak{o}} \mathfrak{s} \mathfrak{s}_{\mathrm{evv}}^{\bullet}, \ddot{\mathfrak{o}} \mathfrak{s} \mathfrak{s}_{\mathrm{odd}}^{\bullet}\right)
\end{aligned}
$$

with six symmetral factors. Three of these, namely $\mathfrak{e s s}_{\mathrm{ev}}^{\bullet}, \ddot{\mathfrak{o s s}} \mathfrak{s v}_{\mathrm{ev}}^{\bullet}$, and $\ddot{\mathfrak{o s s}} \mathfrak{\mathrm { evv }}^{\bullet}$, are highly non-elementary and "even," i.e., simultaneously invariant under neg and pari, which implies that they carry only non-vanishing components of even length. The bimoulds in the next triplet, $\mathfrak{e s s}_{\mathrm{od}}^{\bullet}, \ddot{\mathbf{o}} \mathfrak{s s}_{\mathrm{od}}^{\bullet}$ and $\ddot{\mathbf{o}} \mathfrak{s s}_{\mathrm{odd}}^{\bullet}$, are quite elementary, being given by

$$
\begin{aligned}
& \mathfrak{e s s}_{\mathrm{od}}^{\bullet}=\operatorname{expari}\left(-\frac{1}{2} \mathfrak{E}^{\bullet}\right), \\
& \ddot{\mathbf{o}} \mathfrak{s s}_{\mathrm{od}}^{\bullet}=\operatorname{expari}\left(-\frac{1}{2} \mathfrak{O}^{\bullet}\right) \text {, } \\
& \ddot{\mathbf{o}} \mathfrak{s s _ { \text { odd } } ^ { \bullet }}=\operatorname{expmu}\left(-\frac{1}{2} \mathfrak{O}^{\bullet}\right)
\end{aligned}
$$

or more explicitly

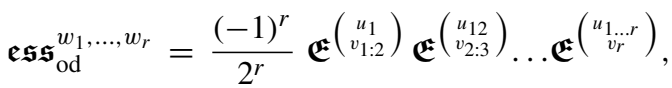

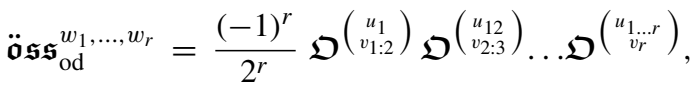

$$
\begin{aligned}
& \ddot{\mathfrak{o}} \mathfrak{s} \mathfrak{s}_{\text {odd }}^{w_{1}, \ldots, w_{r}}=\frac{(-1)^{r}}{2^{r}} \frac{1}{r !} \mathfrak{O}^{w_{1}} \ldots \mathfrak{O}^{w_{r}} .
\end{aligned}
$$

They are also "odd" in the sense of being invertible under pari or neg

$$
\begin{aligned}
\operatorname{invgari}\left(\mathfrak{c s s}_{\text {od }}^{\bullet}\right) & =\operatorname{pari}\left(\mathfrak{e s s}_{\text {od }}^{\bullet}\right)=\operatorname{neg}\left(\mathfrak{e} \mathfrak{s s}_{\text {od }}^{\bullet}\right), \\
\operatorname{invgari}\left(\ddot{\mathfrak{o}} \mathfrak{s s}_{\text {od }}^{\bullet}\right) & =\operatorname{pari}\left(\ddot{\mathfrak{o s s}} \mathfrak{s}_{\text {od }}^{\bullet}\right)=\operatorname{neg}\left(\ddot{\mathfrak{o s s}} \mathfrak{s d}_{\text {od }}^{\bullet}\right), \\
\operatorname{invmu}\left(\ddot{\mathfrak{o}} \mathfrak{s s _ { \text { od } } ^ { \bullet }}\right) & =\operatorname{pari}\left(\ddot{\mathfrak{o}} \mathfrak{s s _ { \text { od } } ^ { \bullet }}\right)=\operatorname{neg}\left(\ddot{\mathfrak{o}} \mathfrak{s s _ { \text { od } } ^ { \bullet }}\right) .
\end{aligned}
$$

Three points deserve attention here.

First, note the presence of a factor $\frac{1}{r !}$ in (137) and its absence in the inflected counterparts (135) and (136).

Second, there is no equivalent to (140) on the $\mathfrak{E}$-side, that is to say, no remarkable $m u$ factorization ${ }^{27}$ of $\mathfrak{e s s}^{\bullet}$, whether of type $m u\left(\mathfrak{e s s}_{\mathrm{evV}}^{\bullet}, \mathfrak{e s s}_{\text {odd }}^{\bullet}\right)$ or of type $m u\left(\mathfrak{s s s}_{\text {odd }}^{\bullet}, \mathfrak{e s s}_{\mathrm{evv}}^{\bullet}\right)$.

\footnotetext{
${ }^{26}$ Universal but by no means elementary: it involves square rooting, which in the case of identity-tangent mappings $f$ generically produces divergence (of "resurgent" type).

${ }^{27}$ i.e., no factorization with at least one elementary factor.
} 
Third, while $\mathfrak{e s s}^{\bullet} / \ddot{\mathbf{o s s}} \mathfrak{s s}^{\bullet}$ are swap-related, $\mathfrak{e s s}_{\text {od }}^{\bullet} / \ddot{\mathbf{o s s}} \mathfrak{s}_{\text {od }}^{\bullet}$ are syap-related and $\mathfrak{e} \mathfrak{s s}_{\mathrm{ev}}^{\bullet} / \ddot{\mathbf{o}} \mathfrak{s s}_{\mathrm{ev}}^{\bullet}$ are not related at all (in any simple way). There would be some justification, therefore, for denoting the odd factor $\mathfrak{o s s}_{\mathrm{ev}}^{\bullet}$ rather than $\ddot{\mathfrak{o s s}} \mathfrak{e}_{\mathrm{ev}}^{\bullet}$, though in a way that too might be confusing. The truth is that this theory is so replete with symmetries that no nomenclature can possibly do justice to them all.

Proposition 3.10 (Even factors: separators) The separators of $\mathfrak{e s s}_{e v}$ are unremarkable 28 but those of $\mathfrak{r i e s s}_{\text {ev }}$ exactly mirror, up to parity, the formulae for riess

$$
\begin{aligned}
\text { gepar.riess } & =1^{\bullet}+\sum_{r \geq 1} 4^{-r} \operatorname{mu}_{r}\left(\mathfrak{O}^{\bullet}\right), \\
\text { hepar.riess } & =\sum_{r \geq 1} 4^{-r} \operatorname{mu}_{\mathrm{r}}\left(\mathfrak{O}^{\bullet}\right) .
\end{aligned}
$$

Proposition 3.11 (Even factors: gari- and gira-dilators) The three identities read

$$
\begin{aligned}
& \text { der.ess } \mathfrak{e v}_{\mathrm{ev}}^{\bullet}=\operatorname{preari}\left(\mathfrak{e s s}_{\mathrm{ev}}^{\bullet}, \mathfrak{d i e s s} \mathfrak{s v}_{\mathrm{ev}}^{\bullet}\right) \text {, } \\
& \operatorname{der} . \ddot{\mathfrak{o}} \mathfrak{s} \mathfrak{s}_{\mathrm{ev}}^{\bullet}=\operatorname{preira}\left(\ddot{\mathfrak{o}} \mathfrak{s} \mathfrak{s}_{\mathrm{ev}}^{\bullet}, \mathfrak{d} \mathfrak{a} \ddot{\mathfrak{o}} \mathfrak{s s} \mathfrak{e v}_{\mathrm{ev}}\right) \text {, }
\end{aligned}
$$

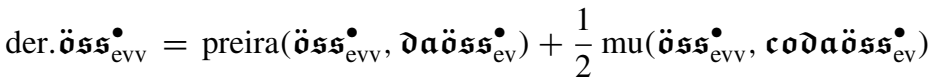

with

$$
\begin{aligned}
& \mathfrak{d i} \mathfrak{s s}_{\mathrm{ev}}^{\bullet}=-\sum_{1 \leq r} \frac{1}{(2 r+1) !} \mathfrak{r} \mathfrak{e}_{2 r}^{\bullet}, \\
& \mathfrak{d} \mathfrak{a} \mathfrak{s s s}_{\mathrm{ev}}^{\bullet}=-\sum_{1 \leq r} \frac{1}{(2 r+1) !} \mathfrak{r} \ddot{o}_{2 r}^{\bullet} \text {, } \\
& \mathfrak{c o d a ̈ a s s} \mathfrak{e v}_{\mathrm{ev}}=\frac{1}{2} \operatorname{expmu}\left(\mathfrak{O}^{\bullet}\right)+\frac{1}{2} \operatorname{expmu}\left(-\mathfrak{O}^{\bullet}\right)-1^{\bullet} \\
& =-\mathfrak{d} \mathfrak{a} \ddot{\mathfrak{o s s}} \mathfrak{s}_{e v}^{\bullet}-\text { anti.d } \mathfrak{a} \ddot{\mathfrak{o}} \mathfrak{s s} \mathfrak{e v}_{\mathrm{e}}^{\bullet} \text {. }
\end{aligned}
$$

Warning: the simultaneous occurrence of $e v / e v v$ in (145) (where $\ddot{\mathbf{o s}} \mathfrak{s}_{\mathrm{evv}}^{\bullet}$ stands side by side with $\mathfrak{d} \mathfrak{a} \ddot{\mathfrak{s}} \mathfrak{s} \mathfrak{s}_{\mathrm{ev}}^{\bullet}$ and $\mathfrak{c o d} \mathfrak{a} \ddot{\mathfrak{o}} \mathfrak{s} \mathfrak{s}_{\mathrm{ev}}^{\bullet}$ ) is no misprint! This awkward jumble in notations is rooted in the nature of our objects and cannot be helped. ${ }^{29}$ We may note, besides, that due to (149) the 'jumbled' identity (145) can be rewritten as follows:

$$
\operatorname{der} . \ddot{\mathfrak{o}} \mathfrak{s} \mathfrak{s}_{\mathrm{evv}}^{\bullet}=\operatorname{irat}\left(\mathfrak{d} \mathfrak{a} \ddot{\mathfrak{o}} \mathfrak{s} \mathfrak{s}_{\mathrm{ev}}^{\bullet}\right) \cdot \ddot{\mathfrak{o}} \mathfrak{s} \mathfrak{s}_{\mathrm{evv}}^{\bullet}+\frac{1}{2} \operatorname{mu}\left(\ddot{\mathfrak{o}} \mathfrak{s} \mathfrak{s}_{\mathrm{evv}}^{\bullet}, \mathfrak{d} \mathfrak{a} \ddot{\mathfrak{o}} \mathfrak{s} \mathfrak{s}_{\mathrm{ev}}^{\bullet}-\operatorname{anti} \cdot \mathfrak{d} \mathfrak{a} \ddot{\mathfrak{o}} \mathfrak{s} \mathfrak{s}_{\mathrm{ev}}^{\bullet}\right)
$$

with id anti rather than $i d+$ anti in front of $\mathfrak{d} \mathfrak{a} \ddot{\mathfrak{o s s}} \mathfrak{e}_{\mathrm{ev}}^{\bullet}$.

Proposition 3.12 (Inverse even factor: gari-dilator) We have two similar looking but logically totally distinct identities

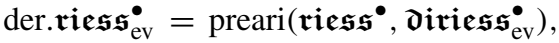

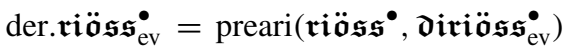

\footnotetext{
${ }^{28}$ The generating functions for gepar $\left(\mathfrak{e s s}_{\mathrm{ev}}^{\bullet}\right)$ and hepar $\left(\mathfrak{e s s}_{\mathrm{ev}}^{\bullet}\right)$ are respectively $\frac{1}{\cosh (x / 2)^{2}}$ and $-\frac{1}{2} \frac{x}{\tanh (x / 2)}$.

${ }^{29}$ The only bimould that would deserve the label $\mathfrak{d} \mathfrak{a} \ddot{\mathbf{o s s}} \mathfrak{e v v}^{\bullet}$ would be the gira-dilator of $\ddot{\mathfrak{o}} \mathfrak{s s _ { \mathrm { evv } } ^ { \bullet }}$, characterized by the identity der. $\ddot{\mathfrak{o}} \mathfrak{s s _ { \mathrm { evv } } ^ { \bullet }}=\operatorname{preira}\left(\ddot{\mathfrak{o}} \mathfrak{s s _ { \mathrm { evv } } ^ { \bullet }}, \mathfrak{d} \mathfrak{a} \ddot{\mathfrak{o}} \mathfrak{s s _ { \mathrm { evv } } ^ { \bullet }}\right)$. That bimould very much exists, of course, but it is thoroughly uninteresting and we can forget about it.
} 
with dilators equal to

$$
\begin{gathered}
\mathfrak{d i x i e s s}_{\mathrm{ev}}^{\bullet}:=+\sum_{r \geq 1} \frac{2^{1-2 r}}{(2 r-1) \cdot(2 r+1)} \mathfrak{r} \mathfrak{e}_{2 r}^{\bullet} \in \text { alternal, } \\
\mathfrak{d i r i o ̈ s} \mathfrak{s}_{\mathrm{ev}}^{\bullet}:=+\sum_{r \geq 1} \frac{2^{1-2 r}}{(2 r-1) \cdot(2 r+1)} \mathfrak{k} \mathfrak{o}_{2 r}^{\bullet} \in \text { alternal }
\end{gathered}
$$

and with the semi-elementary alternals $\mathfrak{k o}_{2 r}^{\bullet}$ defined as in Section 2 but based on the unit $\mathfrak{O}$ instead of $\mathfrak{E}$.

Proposition 3.13 (Even factors: mu-dilators) We have two similar looking but logically rather distinct identities

$$
\begin{aligned}
& \ddot{\mathfrak{o}} \mathfrak{s s ^ { \bullet }}=\operatorname{muu}\left(\ddot{\mathfrak{o v}} \mathfrak{s s _ { \mathrm { ev } }}, \mathfrak{d} \mathfrak{u} \mathfrak{u} \ddot{\mathfrak{o}} \mathfrak{s s _ { \mathrm { ev } }}\right),
\end{aligned}
$$

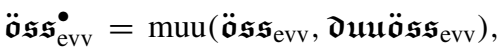

$$
\begin{aligned}
& \mathfrak{d} \mathfrak{u} u \ddot{\mathfrak{o}} \mathfrak{s s}_{\mathrm{ev}}^{\bullet}:=+\sum_{r \geq 1} \alpha_{2 r} \mathfrak{l o}_{2 r}^{\bullet} \in \text { alternal, } \\
& \mathfrak{d} \mathfrak{u} \ddot{\mathbf{o}} \mathfrak{s} \mathfrak{s}_{\mathrm{evv}}^{\bullet}:=+\sum_{r \geq 1} \beta_{2 r} \mathfrak{l o}_{2 r}^{\bullet} \in \text { alternal }
\end{aligned}
$$

with the bilinear product muu defined as in (25) and the same elementary alternals $\mathfrak{l o}_{r}^{\bullet}$ as above. The coefficients $\alpha_{2 r}$ are also the same as in (121) except for the omission of $\alpha_{1}$, but (158) involves new coefficients $\beta_{2 r}$ given by

$$
\sum_{r \geq 1} \beta_{2 r} t^{2 r}:=\frac{t}{e^{t / 2}-e^{-t / 2}}-1=-\frac{1}{24} t^{2}+\frac{7}{5760} t^{4}-\frac{31}{967680} t^{6}+\ldots
$$

Under the polar specialization $\mathfrak{O} \mapsto P a$ the above relations assume a simpler form, with muu replaced by the familiar product $\mathrm{mu}$

$$
\begin{aligned}
& \text { dur.pal }_{\mathrm{ev}}^{\bullet}=\operatorname{mu} .\left(\mathrm{pal}_{\mathrm{ev}}^{\bullet}, \mathrm{dupal}_{\mathrm{ev}}^{\bullet}\right) \text {, } \\
& \text { dur.pal }{ }_{\mathrm{evv}}^{\bullet}=\text { mu. }\left(\text { pal }_{\mathrm{evv}}^{\bullet}, \text { dupal }_{\mathrm{evv}}^{\bullet}\right)
\end{aligned}
$$

and with

$$
\text { dupal }_{\mathrm{ev}}^{\bullet}:=\sum_{r_{*} \geq 1} \alpha_{2 r} \operatorname{lan}_{2 r_{*}}^{\bullet} ; \text { dupal }_{\mathrm{evv}}^{\bullet}:=\sum_{r_{*} \geq 1} \beta_{2 r} \operatorname{lan}_{2 r_{*}}^{\bullet}
$$

relatively to the same elementary alternals $\operatorname{lan}_{r}^{\bullet}$ as in (124).

This concludes our list of "main statements" about the bisymmetrals. For easy reference, we now tabulate the main source functions behind their separators and dilators. 


\section{Table 1: gari-dilators and their Coefficients:}

In all the instances encountered in this section (six in all), we list the identity-tangent diffeomorphisms $f$ with their images in $G A R I_{\mathfrak{r} \mathfrak{e}}$ or $G A R I_{\mathfrak{r} \mathfrak{o}}$ for the unit choice $\mathfrak{E}$ or $\mathfrak{O}$ and the corresponding polar specializations

$$
\left\{f:=x \mapsto x+x \sum a_{n} x^{n}\right\} \mapsto\left\{\mathfrak{f e}^{\bullet}, \mathfrak{f o}^{\bullet}\right\} \text { and }\left\{\mathrm{fi}^{\bullet}, \mathrm{fa}^{\bullet}\right\}
$$

along with the four relevant generating functions

- $f_{0}(x):=x^{-1} f_{\#}(x)=1-\frac{f(x)}{x f^{\prime}(x)}$ : carries the coefficients of the gari-dilators,

- $f_{1}(x):=f^{\prime}(x)$ : carries the coefficients of the first separator gepar,

- $f_{2}(x):=\frac{1}{2} x \frac{f^{\prime \prime}(x)}{f^{\prime}(x)}$ : carries the coefficients of the second separator hepar,

- $f_{3}(x):=\frac{f^{\prime \prime \prime}(x)}{f^{\prime}(x)}-\frac{3}{2}\left(\frac{f^{\prime \prime}(x)}{f^{\prime}(x)}\right)^{2}=$ Schwarzian of $f$ : ought to carry the coefficients of a conjectural third separator (still unknown).

Instance 1 : $\left\{f(x)=1-e^{-x}\right\} \mapsto\left\{\mathfrak{e s s}^{\bullet}, \mathfrak{o s s}^{\bullet}\right\}$ and $\left\{\right.$ pil ${ }^{\bullet}$, pal $\left.{ }^{\bullet}\right\}$,

$$
\begin{aligned}
& f_{0}(x)=\frac{1+x-\exp (x)}{x}=\sum_{1 \leq r} \frac{-1}{(r+1) !} x^{r}, \\
& f_{1}(x)=\exp (-x)=1+\sum_{1 \leq r} \frac{(-1)^{r}}{r !} x^{r}, \\
& f_{2}(x)=-\frac{1}{2} x \\
& f_{3}(x)=-\frac{1}{2} .
\end{aligned}
$$

Instance 2: $\left\{f(x)=\frac{x}{1+\frac{1}{2} x}\right\} \mapsto\left\{\mathfrak{s s s}_{\mathrm{od}}^{\bullet}, \mathfrak{o s s}_{\mathrm{od}}^{\bullet}\right\}$ and $\left\{\right.$ pil $\left.{ }_{\mathrm{od}}^{\bullet}, \mathrm{pal}_{\mathrm{od}}^{\bullet}\right\}$

$$
\begin{aligned}
& f_{0}(x)=-\frac{1}{2} x \\
& f_{1}(x)=\frac{1}{\left(1+\frac{1}{2} x\right)^{2}}, \\
& f_{2}(x)=-\frac{x}{2} \frac{1}{\left(1+\frac{1}{2} x\right)}, \\
& f_{3}(x)=0 .
\end{aligned}
$$

Instance 3 : $\left\{f(x)=2 \tanh \left(\frac{x}{2}\right)\right\} \mapsto\left\{\mathfrak{s s s}_{\mathrm{ev}}^{\bullet}, \mathfrak{o s s}_{\mathrm{ev}}^{\bullet}\right\}$ and $\left\{\mathrm{pil}_{\mathrm{ev}}^{\bullet}, \mathrm{pal}_{\mathrm{ev}}^{\bullet}\right\}$ 


$$
\begin{aligned}
& f_{0}(x)=1-\frac{\sinh (x)}{x}=\sum_{1 \leq r_{*}} \frac{-1}{\left(2 r_{*}+1\right) !} x^{2 r_{*}} \\
& f_{1}(x)=\left(\cosh \left(\frac{x}{2}\right)\right)^{-2}=1-\frac{1}{4} x^{2}+\frac{1}{24} x^{4}-\frac{17}{2880} x^{6}+\frac{31}{40320} x^{8}+\ldots \\
& f_{2}(x)=-\frac{x}{2} \tanh \left(\frac{x}{2}\right)=-\frac{1}{4} x^{2}+\frac{1}{48} x^{4}-\frac{1}{480} x^{6}+\frac{17}{80640} x^{8}+\ldots \\
& f_{3}(x)=-\frac{1}{2}
\end{aligned}
$$

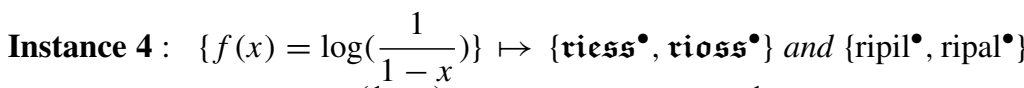

$$
\begin{aligned}
& f_{0}(x)=1+\frac{(1-x)}{x} \log (1-x)=\sum_{1 \leq r} \frac{1}{r(r+1)} x^{r}, \\
& f_{1}(x)=\frac{1}{(1-x)}, \\
& f_{2}(x)=\frac{x}{2} \frac{1}{(1-x)}, \\
& f_{3}(x)=\frac{1}{2} \frac{1}{(1-x)^{2}} .
\end{aligned}
$$

Instance 5: $\left\{f(x)=\frac{1}{1-\frac{1}{2} x}\right\} \mapsto\left\{\operatorname{riess}_{\mathrm{od}}^{\bullet}, \operatorname{rioss}_{\mathrm{od}}^{\bullet}\right\}$ and $\left\{\right.$ ripil $_{\mathrm{od}}^{\bullet}$, ripal $\left.{ }_{\mathrm{od}}^{\bullet}\right\}$

$$
\begin{aligned}
& f_{0}(x)=\frac{1}{2} x \\
& f_{1}(x)=\frac{1}{\left(1-\frac{1}{2} x\right)^{2}}, \\
& f_{2}(x)=\frac{x}{2} \frac{1}{\left(1-\frac{1}{2} x\right)}, \\
& f_{3}(x)=0
\end{aligned}
$$

Instance 6 : $\left\{f(x)=2 \operatorname{arctanh}\left(\frac{x}{2}\right)\right\} \mapsto\left\{\operatorname{riess}_{\mathrm{ev}}^{\bullet}, \operatorname{rioss}_{\mathrm{ev}}^{\bullet}\right\}$ and $\left\{\operatorname{ripil}_{\mathrm{ev}}^{\bullet}\right.$, $\left.\operatorname{ripal}_{\mathrm{ev}}^{\bullet}\right\}$

$$
\begin{aligned}
& f_{0}(x)=1+\left(\frac{1}{x}-\frac{x}{4}\right) \log \left(\frac{1-\frac{1}{2} x}{1+\frac{1}{2} x}\right)=x \sum_{1 \leq r_{*}} \frac{2^{1-2 r_{*}}}{\left(2 r_{*}-1\right)\left(2 r_{*}+1\right)} x^{2 r *}, \\
& f_{1}(x)=\frac{1}{1-\frac{1}{4} x^{2}} \\
& f_{2}(x)=\frac{x^{2}}{4} \frac{1}{\left(1-\frac{1}{4} x^{2}\right)} \\
& f_{3}(x)=\frac{1}{2} \frac{1}{\left(1-\frac{1}{4} x^{2}\right)^{2}} .
\end{aligned}
$$




\section{Table 2: $m u$-dilators and their Coefficients:}

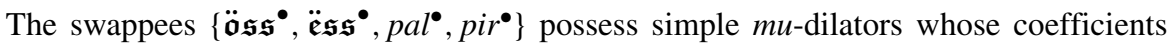
admit the following generating function:

$$
\frac{t}{e^{t}-1}-1=-\frac{1}{2} t+\frac{1}{12} t^{2}-\frac{1}{720} t^{4}+\frac{1}{30240} t^{6}-\frac{1}{120960} t^{8}+\ldots
$$

The even gari-factors $\left\{\ddot{\mathfrak{o}} \mathfrak{s s}_{\mathrm{ev}}^{\bullet}, \ddot{\mathfrak{e}} \mathfrak{s s}_{\mathrm{ev}}^{\bullet}, p a l_{\mathrm{ev}}^{\bullet}, p i r_{\mathrm{ev}}^{\bullet}\right\}$ of these swappees possess simple $m u$ dilators whose coefficients admit the same generating function, minus the first exceptional odd term

$$
\frac{t}{e^{t}-1}-1+\frac{1}{2} t=\frac{1}{12} t^{2}-\frac{1}{720} t^{4}+\frac{1}{30240} t^{6}-\frac{1}{120960} t^{8}+\ldots
$$

Their even $m u$-factors $\left\{\ddot{\mathbf{o}} \mathfrak{s s _ { \mathrm { evv } } ^ { \bullet }}, \ddot{\mathfrak{e}} \mathfrak{s s _ { \mathrm { evv } } ^ { \bullet }}\right.$, pal $\left.\right|_{\mathrm{evv}} ^{\bullet}$ pir $\left.r_{\mathrm{evv}}^{\bullet}\right\}$ also possess simple $m u$-dilators but with coefficients admitting a rather distinct generating function

$$
\frac{t}{e^{t / 2}-e^{-t / 2}}-1=-\frac{1}{24} t^{2}+\frac{7}{5760} t^{4}-\frac{31}{967680} t^{6}+\frac{127}{15482880} t^{8}+\ldots
$$

\section{Polar Bisymmetrals: Proofs}

We shall work mostly with the natural polar specialization $(\mathfrak{E}, \mathfrak{O}) \mapsto(P i, P a)$.

\subsection{Separators of pil $^{\bullet}$ and ripil $^{\bullet}$}

All separator identities in Section 3 result from the general statement:

If $f^{\bullet}$ is the image in the group GARI $I_{\mathfrak{r}}$ of the identity-tangent mapping $f: x \mapsto x+$ $\sum_{1 \leq r} a_{r} x^{r+1}$, then its two separators are of the form

$$
\begin{aligned}
& \text { gepar.fi }{ }^{\mathrm{w}_{1}, \ldots, \mathrm{w}_{r}}=a_{r}^{*} \mathrm{~Pa}^{w_{1}} \ldots \mathrm{Pa}^{w_{r}} \quad \text { with } a_{r}^{*}=(r+1) a_{r}, \\
& \text { hepar.fi }{ }^{\mathrm{w}_{1}, \ldots, \mathrm{w}_{r}}=a_{r}^{* *} \mathrm{~Pa}^{\mathrm{w}_{1}} \ldots \mathrm{Pa}^{\mathrm{w}_{r}} \quad \text { with } \quad \sum_{1 \leq r} a_{r}^{* *} x^{r}:=\frac{x}{2} \frac{f^{\prime \prime(x)}}{f^{\prime}(x)} .
\end{aligned}
$$

To prove (191), we note that the bimould $f i^{\bullet}$, being the image of $f$, has a gari-dilator of the form

$$
\text { der.fi } \mathrm{fi}^{\bullet}=\operatorname{preari}\left(\mathrm{fi}^{\bullet}, \operatorname{difi}^{\bullet}\right) \quad \text { with } \quad \operatorname{difi}^{\bullet}=\sum_{1 \leq r} \alpha_{r} \mathrm{ri}_{\mathrm{r}}^{\bullet}
$$

so that its swappee $f a^{\bullet}$ has a gira-dilator of the form

$$
\text { der.fa }{ }^{\bullet}=\operatorname{preira}\left(\mathrm{fa}^{\bullet}, \text { dafa }^{\bullet}\right) \quad \text { with } \quad \text { dafa }^{\bullet}=\sum_{1 \leq r} \alpha_{r} \operatorname{sra}_{\mathrm{r}}^{\bullet}
$$

with $s r a_{r}^{\bullet}:=$ swap. $r i_{r}^{\bullet}$ and with identical coefficients $\alpha_{r}$ given by

$$
1-\frac{f(x)}{x f^{\prime}(x)}=\sum_{1 \leq r} \alpha_{r} x^{r} .
$$

Due to the very special form of $\operatorname{sra} a_{r}^{\bullet}$ and anti.sra $a_{\mathrm{r}}^{\bullet}$

$$
\text { anti.sra }^{\mathrm{w}_{1}, \ldots, \mathrm{w}_{r}}=P\left(u_{1}+\ldots u_{r}\right) \sum_{1 \leq i \leq r} i \prod_{j \neq i} P\left(u_{j}\right),
$$


the pre-bracket preira in (194) may be replaced by preiwa, which becomes

$$
\text { der.fa }{ }^{\bullet}=\operatorname{preiwa}\left(\mathrm{fa}^{\bullet}, \operatorname{dafa}^{\bullet}\right)=\operatorname{iwat}\left(\mathrm{dafa}^{\bullet}\right) \cdot \mathrm{fa}^{\bullet}+\operatorname{mu}\left(\mathrm{fa}^{\bullet}, \mathrm{dafa}^{\bullet}\right) .
$$

Setting $g e f a^{\bullet}:=m u\left(\right.$ anti.f $\left.f a^{\bullet}, f a^{\bullet}\right)$ and applying the $m u$-derivation der to both sides, we find, in view of (197) and anti.iwat $\left(\mathrm{sra}^{\bullet}\right)=i$ wat $\left(\operatorname{sra}^{\bullet}\right)$.anti

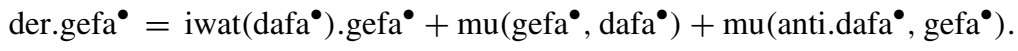

Using the elementary identities

$$
\mathrm{sra}_{\mathrm{r}}^{\bullet}+\text { anti.sra } \mathrm{r}_{\mathrm{r}}^{\bullet}=(r+1) \cdot \mathrm{mu}_{\mathrm{r}}\left(\mathrm{Pa}^{\bullet}\right)
$$

and

$$
\begin{aligned}
& \operatorname{irat}\left(\operatorname{sra}_{p}^{\bullet}\right) \cdot \mathrm{mu}_{\mathrm{q}}\left(\mathrm{Pa}^{\bullet}\right)=\operatorname{iwat}\left(\mathrm{sra}_{\mathrm{p}}^{\bullet}\right) \cdot \mathrm{mu}_{\mathrm{q}}\left(\mathrm{Pa}^{\bullet}\right) \\
& =-(p-q+1) \cdot \mathrm{mu}_{\mathrm{p}+\mathrm{q}}\left(\mathrm{Pa}^{\bullet}\right) \\
& +\mathrm{mu}\left(\mathrm{sra}_{p}^{\bullet}, \mathrm{mu}_{\mathrm{q}}\left(\mathrm{P}^{\bullet}\right)\right) \\
& +\mathrm{mu}\left(\mathrm{mu}_{\mathrm{q}}\left(\mathrm{P}^{\bullet}\right) \text {, anti.sra } \mathrm{p}_{\mathrm{p}}^{\bullet}\right. \text {, }
\end{aligned}
$$

it is but a short step fom (198) to (191).

The proof for hepar runs along similar lines but is more intricate. Since we do not really require the result in the sequel, let us just mention the key step in the argument. Let $\underline{\boldsymbol{r}}=$ $\left\{r_{1}, \ldots, r_{s}\right\}$ denote any non-ordered sequence of $s$ positive integers, and let $f a_{\boldsymbol{r}}^{\bullet}$, resp. lof $a_{\boldsymbol{r}}^{\bullet}$, denote the part of $f a^{\bullet}$, resp. $\operatorname{lof} a^{\bullet}$, that is multilinear in $s r a_{r_{1}}^{\bullet}, \ldots, s r a_{r_{s}}^{\bullet}$. Applying the rules of Section 1.9 we find

$$
\begin{aligned}
\mathrm{fa}_{\underline{\boldsymbol{r}}}^{\bullet} & =a_{r_{1}} \ldots a_{r_{s}} \sum_{\sigma \in \mathfrak{S}(s)} \operatorname{Paj}^{r_{\sigma(1)}, \ldots, r_{\sigma(s)}} \underset{\operatorname{preira}}{\longrightarrow}\left(\operatorname{sra}_{r_{\sigma(1)}}^{\bullet}, \ldots, \operatorname{sra}_{r_{\sigma(s)}}^{\bullet}\right), \\
\operatorname{lofa}_{\underline{\underline{r}}}^{\bullet} & =\sum_{1 \leq m \leq s} \frac{(-1)^{m-1}}{m} \sum_{\underline{\mathbf{r}}^{1} \ldots \underline{\mathbf{r}}^{m}=\underline{\mathbf{r}}} \operatorname{mu}\left(\mathrm{fa}_{\underline{\underline{r}}^{1}}^{\bullet}, \ldots, \mathrm{fa}_{\underline{\mathbf{r}}^{m}}^{\bullet}\right) .
\end{aligned}
$$

Next, consider

$$
\operatorname{rofa}_{\underline{\mathbf{r}}}^{\bullet}=a_{r_{1}} \ldots a_{r_{s}} \sum_{\sigma \in \mathfrak{S}(s)} \operatorname{Paj}^{r_{\sigma(1)}, \ldots, r_{\sigma(s)}} \operatorname{irat}\left(\operatorname{sra}_{r_{\sigma(r)}}^{\bullet}\right) \ldots \operatorname{irat}\left(\operatorname{sra}_{r_{\sigma(2)}}^{\bullet}\right) \operatorname{sra}_{r_{\sigma(1)}}^{\bullet} .
$$

Although $\operatorname{rof}_{\mathbf{r}}^{\bullet}$ has a much simpler (less composite) definition than $\operatorname{lof} a_{\mathbf{r}}^{\bullet}$ and actually differs from it as soon as $r \geq 2$, one can nonetheless show that after pus-averaging the two expressions do coincide:

$$
\sum_{1 \leq k \leq|\boldsymbol{r}|} \operatorname{pus}^{k} \cdot \operatorname{lofa}_{\underline{\mathbf{r}}}^{\bullet} \equiv \sum_{1 \leq k \leq|\boldsymbol{r}|} \operatorname{pus}^{k} \cdot \operatorname{rofa}_{\underline{\mathbf{r}}}^{\bullet}
$$

\subsection{Shape of the gari-Dilators of pil $^{\bullet}$ and ripil $^{\bullet}$}

This is a standard application of the correspondence $f \mapsto f_{\#}$. See Table 1 at the end of the preceding section, where $f_{0}(x) \equiv f_{\#}(x) / x$. See also Section 4 in [4], from (4.11) through (4.17).

\subsection{Bisymmetrality of $\mathrm{pal}^{\bullet} / \mathrm{pil}^{\bullet}$ : First Proof}

This proof strives to be even-handed, in the spirit of dimorphy: it treats $p a l^{\bullet}$ and $p i l^{\bullet}$ in exactly the same way, by relating each to its dilator. So, rather than defining pil ${ }^{\bullet}$ 
from its source mapping $f$ as in Proposition 3.1, we adopt the following, strictly equivalent definition, polar-transposed from Proposition 3.6 and based on the gari-dilator dipil $^{\bullet}$ :

$$
\begin{aligned}
\text { der.pil } & \bullet=\operatorname{preari}\left(\text { pil }{ }^{\bullet}, \operatorname{dipil}^{\bullet}\right) \\
\text { with } \quad \operatorname{dipil}^{\bullet} & :=-\sum_{1 \leq r} \frac{1}{(r+1) !} \mathrm{ri}_{r}^{\bullet} .
\end{aligned}
$$

The alternals $r i_{r}^{\bullet}$ are of course the specialization of $\mathfrak{r} \mathfrak{e}_{r}^{\bullet}$ under $\mathfrak{E} \mapsto P i$.

We then consider a bimould pal $l^{\bullet}$ defined, not as the swappee of pil ${ }^{\bullet}$, but directly and independently, via the $m u$-dilator dupal ${ }^{\bullet}$ :

$$
\begin{aligned}
\text { dur.pal } & \bullet^{\bullet}=\operatorname{mu}\left(\mathrm{pal}^{\bullet}, \text { dupal }^{\bullet}\right) \\
\text { with } \quad \operatorname{dupal}^{\bullet} & :=\sum_{1 \leq r} \alpha_{r} \operatorname{lan}_{r}^{\bullet} \quad\left(\alpha_{r}\right. \text { as in (121)) }
\end{aligned}
$$

with the same Bernoulli coefficients $\alpha_{r}$ as in Proposition 3.8 and with $\operatorname{lan}_{r}^{\bullet}$ being the specialization of $\mathfrak{l e n}_{r}^{\bullet}$ under $\mathfrak{E} \mapsto P a$. See Section 2. Quite explicitly,

$$
\begin{aligned}
\operatorname{lan}_{r}^{\bullet} & =\sum_{1 \leq i \leq r}(-1)^{i-1} \frac{(r-1) !}{(i-1) !(r-i) !} \mathrm{mu}\left(\mathrm{mu}_{i-1}\left(\mathrm{~Pa}^{\bullet}\right), \mathrm{I}^{\bullet}, \mathrm{mu}_{\mathrm{r}-\mathrm{i}}\left(\mathrm{Pa}^{\bullet}\right)\right) \\
& =\operatorname{lu}(\mathrm{I}^{\bullet}, \overbrace{\mathrm{Pa}^{\bullet}, \ldots, \mathrm{Pa}^{\bullet}}^{(r-1)}) .
\end{aligned}
$$

Both dilators dipi $^{\bullet}$ and dupal $\boldsymbol{\bullet}^{\bullet}$ being alternal, it immediately follows that pil $\boldsymbol{l}^{\bullet}$ and $\mathrm{pal}^{\bullet}$ are symmetral: this is obvious from the inversion formulae (36) and (39) and from the symmetrality of the mould $P a j^{\bullet}$ common to both.

So everything now reduces to showing that $\mathrm{pal}^{\bullet}$ is actually the swappee of $\mathrm{pil}^{\bullet}$ or, what amounts to the same, that the system (206) that defines pal ${ }^{\bullet}$ is equivalent to the system

$$
\begin{aligned}
\text { der.pal } & =\operatorname{preira}\left(\mathrm{pal}^{\bullet}, \text { dapal }^{\bullet}\right) \\
& =\operatorname{irat}\left(\operatorname{dapal}^{\bullet}\right) \cdot \mathrm{pal}^{\bullet}+\operatorname{mu}\left(\mathrm{pal}^{\bullet}, \operatorname{dapal}^{\bullet}\right) \\
\text { with } \quad \operatorname{dapal}^{\bullet} & :=-\sum_{1 \leq r} \frac{1}{(r+1) !} \operatorname{sra}_{\mathrm{r}}^{\bullet} \quad\left(\operatorname{sra}_{\mathrm{r}}^{\bullet}:=\text { swap.ri }_{\mathrm{r}}^{\bullet}\right)
\end{aligned}
$$

deduced under the swap transform from the system (205) that defines pil ${ }^{\bullet}$.

Before taking that one last step, let us recall the universal relation (27) between the gira-dilator $d a S^{\bullet}$ and the $m u$-dilator $d u S^{\bullet}$ of a given $S^{\bullet}$ :

$$
\text { der.duS } S^{\bullet}-\operatorname{dur} . \operatorname{da} S^{\bullet}+\operatorname{lu}\left(\operatorname{da} S^{\bullet}, \operatorname{du}^{\bullet}\right)-\operatorname{irat}\left(\operatorname{da} S^{\bullet}\right) \cdot \operatorname{du} S^{\bullet}=0 .
$$

Specializing the triplet $\left\{S^{\bullet}, d a S^{\bullet}, d u S^{\bullet}\right\}$ to the triplet $\left\{\right.$ pal $\left.l^{\bullet}, \operatorname{dapal}^{\bullet}, d u p a l^{\bullet}\right\}$, we get

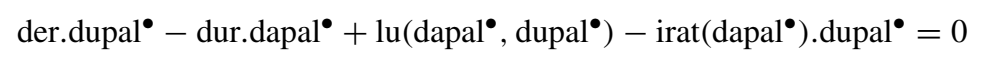

which, as observed in the universal case (cf. Section 1 ), determines dapal ${ }^{\bullet}$ in terms of dupal $^{\bullet}$ and vice versa.

Now, this appealingly symmetrical and winningly simple relation (209) involves only elementary monomials $P a($.$) and readily follows from the basic identities (199), (200) and$ (207).

This establishes beyond cavil that the symmetral bimould pil ${ }^{\bullet}$ as defined by (205) and the equally symmetral bimould pal ${ }^{\bullet}$ as defined by (206) are mutual swappees. 
Remark This last identity (209) is totally rigid in the sense that if we tinker with the common coefficients $-1 /(r+1)$ ! of dipil ${ }^{\bullet}$ and dapal ${ }^{\bullet}$, there is no way we can adjust the coefficients $\alpha_{r}$ of $d u p a l^{\bullet}$ to salvage (209). This rigidity will stand us in good stead in [5] for unraveling the structure of the trigonometric bisymmetrals $t a l^{\bullet} /$ til $^{\bullet}$. For a foretaste, see Section 17 infra.

\subsection{Bisymmetrality of $\mathrm{pal}^{\bullet} / \mathrm{pil}^{\bullet}$ : Second Proof}

This alternative proof is more roundabout ${ }^{30}$ but makes up for it by yielding valuable extra information. We now start from pil ${ }^{\bullet}$ and its gari-inverse ripil ${ }^{\bullet}$, which are automatically symmetral by construction. The challenge is to show that $p a l^{\bullet}$ (now defined derivatively, as the swappee of $\mathrm{pil}^{\bullet}$ ) is also symmetral or, what amounts to the same but turns out to be easier, that its gari-inverse ripal $^{\bullet}$ is symmetral. The key here is to compare ripal ${ }^{\bullet}$ with the swappee rapal $^{\bullet}$ of ripil $^{\bullet}$, which may be also be viewed as the gira-inverse of pal (hence the prefix "ra"). According to (10), ripal is also the ras-transform of rapal $^{\bullet}$ :

$$
\text { ripal }^{\bullet}=\text { ras.rapal }^{\bullet}:=\text { invgari.swap.invgari.swap.rapal }{ }^{\bullet} .
$$

The following picture sums up the situation:

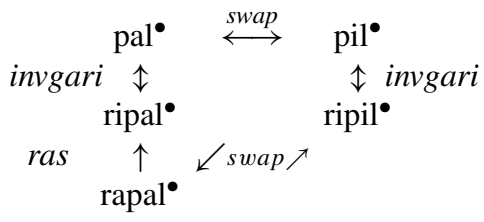

In view of (9), we also have

$$
\begin{aligned}
\operatorname{rash}_{\text {rapal }}{ }^{\bullet} & =\operatorname{mu}\left(\text { corapal }^{\bullet}, \text { rapal }^{\bullet}\right) \quad \text { with } \\
\text { corapal }^{\bullet} & =\text { push.swap.invmu.swap.rapal }
\end{aligned}
$$

Replacing push by its definition (439) in (212) and using the fact that ripil ${ }^{\bullet}$, being symmetral, is $m u$-invertible under pari.anti, we get successively

$$
\begin{aligned}
\text { corapal }^{\bullet} & =\text { neg.anti.swap.anti.swap.swap.invmu.swap.rapal } \\
& =\text { neg.anti.swap.anti.invmu.ripil } \\
& =\text { neg.anti.swap.anti.anti.pari.ripil } \\
& =\text { neg.anti.swap.pari.ripil } \\
& =\text { anti.swap.neg.pari.ripil } \\
& =\text { anti.swap.ripil } \\
& =\text { anti.rapal } \\
&
\end{aligned}
$$

So we end up with

$$
\begin{aligned}
\operatorname{corapal}^{\bullet} & =\operatorname{mu}(\text { anti.rapal } \\
& =\operatorname{gepar}\left(\text { ripil }^{\bullet}\right) \\
& =\operatorname{pac}^{\bullet} \quad(\text { due to }(111))
\end{aligned}
$$

\footnotetext{
${ }^{30}$ Before starting, the reader may have a look at the overall logical scheme as pictured at the end of the paragraph Section 4.4 .
} 
with an elementary $p a c^{\bullet}$ that admits an equally elementary gani-inverse nipac $c^{\bullet}$

$$
\begin{aligned}
\operatorname{pac}^{w_{1}, \ldots, w_{r}} & =\prod_{1 \leq i \leq r} P\left(u_{i}\right), \\
\operatorname{nipac}^{w_{1}, \ldots, w_{r}} & =(-1)^{r} \prod_{1 \leq i \leq r} P\left(u_{i}+\ldots+u_{r}\right), \\
\operatorname{gani}\left(\operatorname{pac}^{\bullet}, \operatorname{nipac}^{\bullet}\right) & =1^{\bullet} .
\end{aligned}
$$

Thus, in view of (8), we go from ripal $^{\bullet}$ to rapal $^{\bullet}$ and back via the relations

$$
\begin{aligned}
& \operatorname{ganit}\left(\mathrm{pac}^{\bullet}\right) \cdot \operatorname{ripal}^{\bullet}=\text { rapal }^{\bullet}, \\
& \operatorname{ganit}\left(\operatorname{nipac}^{\bullet}\right) \cdot \operatorname{rapal}^{\bullet}=\operatorname{ripal}^{\bullet} .
\end{aligned}
$$

Now, it is an easy matter to check $^{31}$ that

$$
\begin{aligned}
& \text { ganit }\left(\mathrm{pac}^{\bullet}\right) \text { :alternal //symmetral } \longrightarrow \text { alternul//symmetrul, } \\
& \text { ganit(nipac } \left.{ }^{\bullet}\right): \text { alternul//symmetrul } \longrightarrow \text { alternal//symmetral } \text {. }
\end{aligned}
$$

Let us now write down the dilator identity for ripil ${ }^{\bullet}$ (see (151)-(153)) and the logically equivalent identity for the swappee rapal ${ }^{\bullet}$ :

$$
\begin{aligned}
& \text { der.ripil }^{\bullet}=\operatorname{preari}_{\left(\text {ripil }^{\bullet}, \text { diripil }^{\bullet}\right) \quad \text { with } \text { diripil }^{\bullet}}=\sum_{1 \leq r} \frac{1}{r \cdot(r+1)} \mathrm{ri}_{r}^{\bullet}, \\
& \text { der.rapal }^{\bullet}=\operatorname{preira}\left(\text { rapal }^{\bullet}, \text { darapal }^{\bullet}\right) \text { with } \text { darapal }^{\bullet}=\sum_{1 \leq r} \frac{1}{r \cdot(r+1)} \operatorname{sra}_{r}^{\bullet} .
\end{aligned}
$$

As usual, $\operatorname{sra}_{r}^{\bullet}:=$ swap.ri $i_{r}^{\bullet}$. More explicitly,

$$
\operatorname{sra}_{r}^{w_{1}, \ldots, w_{r}}=\frac{\sum(r+1-i) u_{i}}{u_{1} \ldots u_{r}\left(u_{1}+\ldots u_{r}\right)} .
$$

From that we infer the shuffle identity

$$
\begin{aligned}
\sum_{w \in \operatorname{sha}\left(w^{1}, w^{2}\right)} \operatorname{esra}_{r}^{w} & \equiv \operatorname{esra}_{\mathrm{r}_{1}}^{w^{1}} \operatorname{expa}_{r_{2}}^{w^{2}}+\operatorname{expa}_{r_{1}}^{w^{1}} \operatorname{esra}_{r_{2}}^{w^{2}} \quad \text { with } \\
\operatorname{esra}_{r}^{\bullet} & :=\frac{1}{(r+1) !} \text { dur.sra }_{\mathrm{r}}^{\bullet} \\
\operatorname{expa}_{r}^{\bullet} & :=\operatorname{expmu}\left(\mathrm{Pa}^{\bullet}\right),
\end{aligned}
$$

which in turn easily implies that the dilator darapal ${ }^{\bullet}$, as given by (239), is altern $u l^{32}$ Now, if from "darapal $\in$ altern $u l^{\bullet}$ " we could directly deduce "rapal $\in$ symmetrul," life would be easy: we could, applying (227) and (229), immediately conclude that ripal ${ }^{\bullet}$ and therefore

\footnotetext{
${ }^{31}$ Especially in the form (228). For details about the "twisted symmetries" alternil/symmetril and alternul/symmetrul, see [4], Section 3.5.

${ }^{32}$ This fact is already mentioned in [4], in "universal mode": see (4.6) p. 73.
} 
$p a l^{\bullet}$ are symmetral, and be done with it. Unfortunately, we cannot ${ }^{33}$ —at least not directlyand must take the detour through the dilators darapal ${ }^{\bullet}$ and diripal ${ }^{\bullet}$.

So our goal now is to go from the proven identity (231) to an identity of the form

$$
\begin{aligned}
& \text { der.ripal }{ }^{\bullet}=\operatorname{preari}\left(\text { ripal }^{\bullet}, \text { diripal }^{\bullet}\right) \quad \text { with }
\end{aligned}
$$

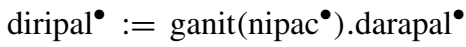

and from there to the identity

$$
\text { der.ripal }^{\bullet}=\operatorname{preari}_{\left(\text {ripal }^{\bullet}, \text { diripal }^{\bullet}\right)} \quad \text { with } \text { diripal }^{\bullet}=\sum_{1 \leq r} \frac{1}{r .(r+1)} \mathrm{ha}_{r}^{\bullet} .
$$

To deal with the first step, let us parse the identities (231) and (236) respectively as $A_{1}+A_{2}=0$ and $B_{1}+B_{2}=0$ with

$$
\begin{array}{ll}
A_{1}:=\left(-\operatorname{der}+\operatorname{irat}\left(\operatorname{darapal}^{\bullet}\right)\right) \cdot \operatorname{rapal}^{\bullet} & A_{2}:=\operatorname{mu}\left(\mathrm{rapal}^{\bullet}, \text { darapal }^{\bullet}\right), \\
B_{1}:=\left(-\operatorname{der}+\operatorname{arit}\left(\operatorname{diripal}^{\bullet}\right)\right) \cdot \operatorname{ripal}^{\bullet} \quad B_{2}:=\operatorname{mu}\left(\text { ripal }^{\bullet}, \text { diripal }^{\bullet}\right)
\end{array}
$$

and then check that

$$
\begin{aligned}
& \operatorname{ganit}\left(\operatorname{nipac}^{\bullet}\right) \cdot A_{1}=B_{1}, \\
& \operatorname{ganit}\left(\operatorname{nipac}^{\bullet}\right) \cdot A_{2}=B_{2} .
\end{aligned}
$$

The relation (241) is simply the definition of diripal ${ }^{\bullet}$ : see (236), second line. To prove the non-trivial part, namely

$$
\text { ganit(nipac } \left.{ }^{\bullet}\right) \cdot A_{1}=B_{1}
$$

we apply to rapal $^{\bullet}$ both terms of the operator identity

$$
\begin{array}{r}
\left.\operatorname{ganit}\left(\operatorname{nipac}^{\bullet}\right) \cdot\left[-\operatorname{der}+\operatorname{irat}(\operatorname{darapal})^{\bullet}\right)\right] \equiv \\
\left.\left[-\operatorname{der}+\operatorname{arit}\left(\operatorname{ganit}^{\bullet} \operatorname{nipac}^{\bullet}\right) \cdot \operatorname{darapal} l^{\bullet}\right)\right] \cdot \operatorname{ganit}\left(\operatorname{nipac}^{\bullet}\right),
\end{array}
$$

which is easier to check in this equivalent formulation: ${ }^{34}$

$$
\begin{array}{r}
{\left[-\operatorname{der}+\operatorname{irat}\left(\operatorname{darapal} l^{\bullet}\right)\right] \cdot \operatorname{ganit}\left(\operatorname{pac}^{\bullet}\right) \equiv} \\
\operatorname{ganit}\left(\operatorname{pac}^{\bullet}\right) \cdot\left[-\operatorname{der}+\operatorname{arit}\left(\operatorname{ganit}\left(\operatorname{nipac}^{\bullet}\right) \cdot \operatorname{darapal} l^{\bullet}\right)\right] .
\end{array}
$$

Thus, the $m u$-isomorphism ganit(nipac ${ }^{\bullet}$ ) takes us from (231) to (236), thereby establishing the latter identity, with a dilator diripal ${ }^{\bullet}$ which, being the image under ganit(nipac ${ }^{\bullet}$ ) of the altern $u$ d darapal ${ }^{\bullet}$, is automatically alternal. This in turn immediately implies that ripal $^{\bullet}$ and $\mathrm{pal}^{\bullet}$ are symmetral. It also implies, in view of (227), that rapal $^{\bullet}$ is symmetrul-the very property, recall, that we could not directly derive from "darapal $\in$ altern $u$ l".

This completes our second, less direct proof of the bisymmetrality of pal $\boldsymbol{l}^{\bullet}$ pil ${ }^{\bullet}$. What it does not do, though, is to prove that our definitely alternal bimould diripil ${ }^{\bullet}$ admits the

\footnotetext{
${ }^{33} \mathrm{To}$ do that directly, we would require the alternulity of the gari-dilator dirapal ${ }^{\bullet}$ of rapal ${ }^{\bullet}$ (not considered here) rather than the alternulity of its gira-dilator darapal ${ }^{\bullet}$ (considered!). Extreme caution is called for here; great care must be taken to distinguish between the various dilators: diripil ${ }^{\bullet}$ (linked to ripil), diripal ${ }^{\bullet}$ (linked to ripal), and the pair darapal ${ }^{\bullet} /$ dirapal $^{\bullet}$ (both linked to rapal $^{\bullet}$, but in different ways). Always pay close attention to the vowels and their placement: no agglutinative language with vocalic alternation could beat flexion theory for fiendish intricacy! But that is no fault of ours. That is just the way things are, and there is no point in carping.

${ }^{34}$ These are "rigid" identities, strictly dependent on the nature of the inputs: if we were to modify the definition of darapal ${ }^{\bullet}$ by, say, modifying the coefficients of $s r a r$ in (231), we would have to simultaneously modify the pair $\mathrm{pac}^{\bullet}$, nipac $\bullet^{\bullet}$ of gani-inverse elements.
} 
exact expansion (237), with $h a_{r}^{\bullet}$ the polar specialization of $\mathfrak{h} \mathfrak{e}_{r}^{\bullet}$ under $\mathfrak{E} \mapsto P a$. To rigorously establish this non-essential, but very nice extra bit of information unfortunately requires rather lengthy and tedious, though in a sense elementary calculations. One way to proceed is to start from the expansion (231) of darapal $^{\bullet}$; to apply ganit(nipac ${ }^{\bullet}$ ) to each $\operatorname{sra}_{r}{ }^{\bullet}$ separately, resulting in a bimould $\operatorname{hasra}_{r}^{\bullet}$ with infinitely many non-vanishing components:

$$
\operatorname{hasra}_{r}^{\bullet}:=\sum_{r \leq r_{*}} \text { hasra }_{r, r_{*}}^{\bullet} \text { with } \text { hasra }_{r, r_{*}}^{\bullet} \in \mathrm{BIMU}_{r_{*}} .
$$

One may then expand each hasra ${ }_{r, r_{*}}$ in the standard basis of $F_{l e x}(P a)$, where it admits a rather simple, highly lacunary projection; and eventually piece everything together inside the double sum

$$
\sum_{1 \leq r \leq r_{*}} \frac{1}{r .(r+1)} \text { hasra }_{r, r_{*}}^{\bullet} \equiv \frac{1}{r_{*}\left(r_{*}+1\right)} \mathrm{ha}_{r_{*}}^{\bullet} .
$$

The combinatorially minded reader may fill in the dots. ${ }^{35}$

To conclude, let us sum up the various steps of the whole argument ( - our second bisymmetrality proof-) with the number of stars alongside each arrow reflecting the trickiness of the corresponding implication:

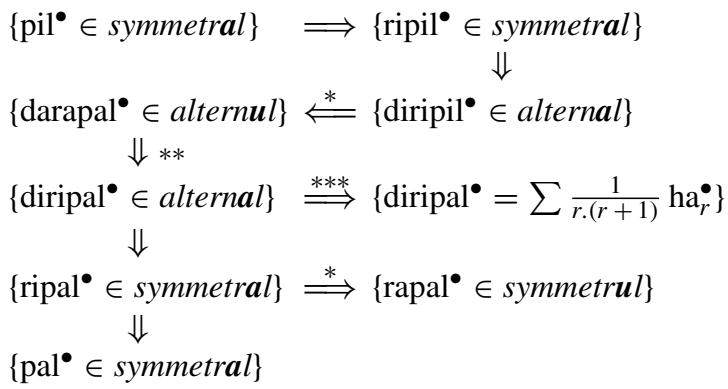

\subsection{Even and Odd Factors of $p^{\circ} l^{\bullet} / p^{\circ} l^{\bullet}$}

We must first establish the three factorizations (129), (130), (131). Despite their air of kinship, they are in fact quite distinct, and must be dealt with separately. Under our preferred polar specialization $(\mathfrak{E}, \mathfrak{O}) \mapsto(P i, P a)$, they become respectively:

$$
\begin{aligned}
& \mathrm{pil}^{\bullet}=\operatorname{gari}\left(\mathrm{pil}_{\mathrm{od}}^{\bullet}, \mathrm{pil}_{\mathrm{ev}}^{\bullet}\right) \quad \text { with } \quad \operatorname{pil}_{\mathrm{od}}^{\bullet}=\operatorname{expari}\left(-\frac{1}{2} \mathrm{Pi}^{\bullet}\right), \\
& \mathrm{pal}^{\bullet}=\operatorname{gari}\left(\mathrm{pal}_{\mathrm{od}}^{\bullet}, \operatorname{pal}_{\mathrm{ev}}^{\bullet}\right) \quad \text { with } \quad \operatorname{pal}_{\mathrm{od}}^{\bullet}=\operatorname{expari}\left(-\frac{1}{2} \mathrm{~Pa}^{\bullet}\right), \\
& \mathrm{pal}^{\bullet}=\mathrm{mu}\left(\mathrm{pal}_{\mathrm{evv}}^{\bullet}, \mathrm{pal}_{\mathrm{odd}}^{\bullet}\right) \quad \text { with } \quad \mathrm{pal}_{\mathrm{odd}}^{\bullet}=\operatorname{expmu}\left(-\frac{1}{2} \mathrm{~Pa}^{\bullet}\right) \text {. }
\end{aligned}
$$

(i) The first factorization (247) merely reflects the factorization $f=f_{\mathrm{od}} \circ f_{\mathrm{ev}}$ of the source diffeomorphisms. Explicitly,

$$
f(x)=1-e^{-x} \quad ; \quad f_{\mathrm{od}}(x)=\frac{x}{1-\frac{1}{2} x} \quad ; \quad f_{\mathrm{ev}}(x)=2 \frac{e^{x / 2}-e^{-x / 2}}{e^{x / 2}+e^{-x / 2}} .
$$

\footnotetext{
${ }^{35}$ There exist alternative strategies, like applying ganit(nipac ${ }^{\bullet}$ ) to $s r a_{r}^{\bullet}$ as (indirectly) defined by (231) and summing, not in $i$ and then $r$ as above, but rather in $r$ and then $i$, but all these approaches seem to lead to calculations of roughly the same complexity and tediousness.
} 
Of course, as a function, $f_{\mathrm{ev}}(x)$ is odd and $f_{\mathrm{od}}(x)$ is neither odd nor even, but what matters in this context is that the quotient $f_{\mathrm{ev}}(x) / x$ should carry only even powers of $x$ and that $f_{\text {od }}(\bullet)$ should admit $-f_{\text {od }}(-\bullet)$ as its reciprocal mapping.

(ii) The second factorization (248) is less immediate to derive. We first observe that if we specialize $\mathfrak{E}$ to $P a$ rather than $P i$, we get instead of (247) the following factorization:

$$
\operatorname{par}^{\bullet}=\operatorname{gari}\left(\operatorname{par}_{\mathrm{od}}^{\bullet}, \operatorname{par}_{\mathrm{ev}}^{\bullet}\right) \quad \text { with } \quad \operatorname{par}_{\mathrm{od}}^{\bullet}=\operatorname{expari}\left(-\frac{1}{2} \mathrm{~Pa}^{\bullet}\right)
$$

Anticipating on the key result of Section 8 below about the canonical factorization of bisymmetrals, we may note that the two exceptional (i.e., non-neg-invariant) bisymmetrals $\mathrm{pal}^{\bullet}$ and $\mathrm{par}^{\bullet}$ necessarily coincide up to gari-postcomposition by a regular (i.e., simultaneously neg- and pari-invariant) bisymmetral, which we may call $\mathrm{ral}^{\bullet}$, and whose first three components $\mathrm{ral}_{1}^{\bullet}, \mathrm{ral}_{2}^{\bullet}, \mathrm{ral}_{3}^{\bullet}$, as well as all later components of odd length, necessarily vanish. In other words

$$
\operatorname{pal}^{\bullet}=\operatorname{gari}\left(\operatorname{par}^{\bullet}, \operatorname{ral}^{\bullet}\right)=\operatorname{gari}\left(\operatorname{par}_{\mathrm{od}}^{\bullet}, \operatorname{par}_{\mathrm{ev}}^{\bullet}, \operatorname{ral}^{\bullet}\right) .
$$

But this is exactly the sought-after factorization (248), with explicit factors:

$$
\begin{aligned}
& \mathrm{pal}_{\mathrm{od}}^{\bullet}=\operatorname{par}_{\mathrm{od}}^{\bullet}=\operatorname{expari}\left(-\frac{1}{2} \mathrm{~Pa}^{\bullet}\right), \\
& \operatorname{pal}_{\mathrm{ev}}^{\bullet}=\operatorname{gari}\left(\operatorname{par}_{\mathrm{ev}}^{\bullet}, \operatorname{ral}^{\bullet}\right) \text {. }
\end{aligned}
$$

(iii) The third factorization (249) is rather special in being a $m u$-factorization incongruously arising out of a purely gari-gira context. ${ }^{36}$ The quickest way to derive it is to assume the (already doubly established) bisymmetrality of $\mathrm{pal}^{\bullet} / \mathrm{pil}^{\bullet}$, then to define the would-be even factor $p a l_{\mathrm{evv}}^{\bullet}$ via (249) in terms of $p a l^{\bullet}$ and $p a l_{\text {odd }}^{\bullet}$; and then to check its evenness. Injecting the factor $p a l_{\mathrm{evv}}^{\bullet}$ so defined into the first separator identity:

$$
\text { gepar.pil } \bullet^{\bullet} \operatorname{mu}\left(\text { anti.pal } l^{\bullet}, \text { pal }^{\bullet}\right)=\operatorname{expmu}\left(-P a^{\bullet}\right),
$$

we find at once

$$
\mathrm{mu}\left(\text { anti.pal }{ }_{\mathrm{evv}}^{\bullet}, \mathrm{pal}_{\mathrm{evv}}^{\bullet}\right)=1
$$

and hence

$$
\text { invmu.pal }{ }_{\mathrm{evv}}^{\bullet}=\text { anti.pal }_{\mathrm{evv}}^{\bullet} \text {. }
$$

But we have defined $\mathrm{pal}_{\mathrm{evv}}^{\bullet}$ as the $m u$-product of $\mathrm{pal}{ }^{\bullet}$, which we have shown to be symmetral, and of $\operatorname{expmu}\left(\frac{1}{2} \mathrm{~Pa}^{\bullet}\right)$, also clearly symmetral. So $p a l_{\mathrm{evv}}^{\bullet}$ is itself symmetral, and as such $m u$-invertible under pari.anti. Therefore

$$
\text { invmu.pal }{ }_{\mathrm{evv}}^{\bullet}=\text { pari.anti.pal }_{\mathrm{evv}}^{\bullet} \text {. }
$$

Comparing (257) and (258), we see that $p a l_{\mathrm{evv}}^{\bullet}$ is pari-invariant, and so neg-invariant as well, and therefore truly even.

Properties of $\boldsymbol{p a l}_{\mathrm{ev}}^{\bullet}$ and $\boldsymbol{p a l} \mathbf{e}_{\mathrm{evv}}^{\bullet}$ In our preferred polar specialization, the identities (143), (144), (145) become

$$
\begin{aligned}
& \text { der.pil }{ }_{\mathrm{ev}}^{\bullet}=\operatorname{preari}\left(\text { pil }_{\mathrm{ev}}^{\bullet}, \operatorname{dipil}_{\mathrm{ev}}^{\bullet}\right) \text {, } \\
& \text { der.pal }_{\mathrm{ev}}^{\bullet}=\operatorname{preira}\left(\mathrm{pal}_{\mathrm{ev}}^{\bullet}, \operatorname{dapal}_{\mathrm{ev}}^{\bullet}\right) \text {, } \\
& \text { der.pal } \bullet_{\mathrm{evv}}^{\bullet}=\operatorname{preira}\left(\mathrm{pal}_{\mathrm{evv}}^{\bullet}, \operatorname{dapal}_{\mathrm{ev}}^{\bullet}\right)+\frac{1}{2} \operatorname{mu}\left(\text { pal }_{\mathrm{evv}}^{\bullet}, \operatorname{codapal}_{\mathrm{ev}}^{\bullet}\right)
\end{aligned}
$$

\footnotetext{
${ }^{36}$ For a tentative mitigation of this "incongruity," see Section 1.11 supra.
} 
with the unavoidable ev/evv jumble in (261) and with dilators given by

$$
\begin{aligned}
& \operatorname{dipil}_{\mathrm{ev}}^{\bullet}:=-\sum_{1 \leq r} \frac{1}{(2 r+1) !} \mathrm{ri}_{2 r}^{\bullet}, \\
& \text { dapal }_{\mathrm{ev}}^{\bullet}:=-\sum_{1 \leq r} \frac{1}{(2 r+1) !} \operatorname{sra}_{2 r}^{\bullet}, \quad\left(\operatorname{sra}_{\mathrm{r}}^{\bullet}:=\text { swap.ri }_{\mathrm{r}}^{\bullet}\right) \\
& \operatorname{codapal}_{\mathrm{ev}}^{\bullet}:=\frac{1}{2} \operatorname{expmu}\left(\mathrm{Pa}^{\bullet}\right)+\frac{1}{2} \operatorname{expmu}\left(-\mathrm{Pa}^{\bullet}\right)-1^{\bullet} \\
&=- \text { dapal }_{\mathrm{ev}}^{\bullet}-\text { anti.dapal } \\
& \text { evv }
\end{aligned}
$$

The identity (259) simply reflects the form of the preimage $f_{\#}$ of the gari-dilator. See $f_{0}:=x^{-1} f_{\#}$ in (172).

The identity (260) is the mechanical transposition of (259) under the involution swap.

To establish the last identity (261), we must start, not from (260), but from the corresponding relation for $\mathrm{pal}^{\bullet}$, which reads

$$
\text { der.pal }{ }^{\bullet}=\operatorname{preira}\left(\text { pal }^{\bullet}, \text { dapal }^{\bullet}\right) \quad \text { with } \quad \text { dapal }^{\bullet}:=-\sum_{1 \leq r} \frac{1}{(r+1) !} \operatorname{sra}_{r}^{\bullet} .
$$

To declumsify our notations, we set $^{37}$

$$
\begin{aligned}
& B:=-\sum_{r \text { even }} \frac{1}{(r+1) !} \operatorname{sra}_{r}^{\bullet} \quad ; \quad C:=-\sum_{r \text { odd }} \frac{1}{(r+1) !} \operatorname{sra}_{r}^{\bullet}, \\
& A:=B+C ; \quad A^{*}:=B-C, \\
& a:=\mathrm{pal}^{\bullet} \quad ; \quad b:=\mathrm{pal}_{\mathrm{evv}}^{\bullet} \quad ; \quad c:=\mathrm{pal}_{\mathrm{odd}}^{\bullet}
\end{aligned}
$$

Furthermore, we shall denote the $m u$-product by a simple dot "." We shall also abbreviate $\operatorname{irat}(A), \operatorname{irat}(B)$ etc. as $\bar{A}, \bar{B}$ etc. Lastly, stars in upper (resp. lower) index position will stand for the involution pari (resp. anti).

With these compact notations, the relation (266) we want to establish reads

$$
\mathcal{R}:=-\operatorname{der}(b . c)+\bar{B} b+b \cdot B-\frac{1}{2} B-\frac{1}{2} B_{*} \equiv 0 .
$$

Using the fact that $\operatorname{der}, \bar{A}, \bar{B}$ etc. are $m u$-derivations, we see that $\mathcal{R}$ may be decomposed as

$$
\mathcal{R}=\mathcal{R}_{1} \cdot c^{-1}+\mathcal{R}_{1}^{*} \cdot c-b \cdot \mathcal{R}_{2}-b \cdot \mathcal{R}_{2}^{*}
$$

with

$$
\begin{aligned}
& \mathcal{R}_{1}:=-\operatorname{der}(b \cdot c)+\bar{A}(b \cdot c)+b \cdot c \cdot A, \\
& \mathcal{R}_{1}^{*}:=-\operatorname{der}\left(b \cdot c^{-1}\right)+\bar{A}^{*}\left(b \cdot c^{-1}\right)+b \cdot c^{-1} \cdot A^{*}, \\
& \mathcal{R}_{2}:=(\bar{A} c) \cdot c^{-1}+c \cdot A \cdot c^{-1}-\frac{1}{2} A+\frac{1}{2} A_{*}-\frac{1}{2} P a^{\bullet}, \\
& \mathcal{R}_{2}^{*}:=\left(\bar{A}^{*} c^{-1}\right) \cdot c+c^{-1} \cdot A^{*} \cdot c-\frac{1}{2} A^{*}+\frac{1}{2} A_{*}^{*}+\frac{1}{2} P a^{\bullet} .
\end{aligned}
$$

Let us now show that $\mathcal{R}_{1} \equiv \mathcal{R}_{1}^{\bullet} \equiv \mathcal{R}_{2} \equiv \mathcal{R}_{2}^{*} \equiv 0$. The identities $\mathcal{R}_{1}^{*} \equiv 0$ and $\mathcal{R}_{2}^{*} \equiv 0$ follow respectively from $\mathcal{R}_{1} \equiv 0$ and $\mathcal{R}_{2} \equiv 0$ under pari, and the identity $\mathcal{R}_{1} \equiv 0$ is none other than (266). So the only thing left to check is $\mathcal{R}_{2} \equiv 0$. To do this, we apply the

\footnotetext{
${ }^{37}$ Note in passing that $B$ is the gira-dilator of $b$, but that $C$ has nothing to do with the gira-dilator of $c$.
} 
derivation rule (200) and then the simplification rule (199) to show that in the expression $(\bar{A} c) . c^{-1}+c . A . c^{-1}$ all "intermediary terms," i.e., all terms of the form

$$
\mathrm{mu}\left(\mathrm{mu}_{r_{1}}\left(\mathrm{~Pa}^{\bullet}\right), \mathrm{sra}_{r_{2}}^{\bullet}, \mathrm{mu}_{r_{3}}\left(\mathrm{~Pa}^{\bullet}\right)\right) \text { or } \mathrm{mu}\left(\mathrm{mu}_{r_{1}}\left(\mathrm{~Pa}^{\bullet}\right), \text { anti.sra } r_{r_{2}}^{\bullet}, \mathrm{mu}_{r_{3}}\left(\mathrm{~Pa}^{\bullet}\right)\right)
$$

with $r_{1} \neq 0, r_{2} \geq 2, r_{3} \neq 0$ disappear, leaving only "extreme terms" that cancel out with the terms from $-1 / 2 A+1 / 2 A^{*}$, plus of course pure $m u$-powers of $P a^{\bullet}$, which also cancel out. This establishes $\mathcal{R} \equiv 0$.

\subsection{Properties of ripal $_{\mathrm{ev}}^{\bullet}$}

Applying the identity (44) for dilator composition to the factorization

$$
\operatorname{ripal}_{\mathrm{ev}}^{\bullet}=\operatorname{gari}\left(\text { ripal }^{\bullet}, \text { pal }_{\text {od }}^{\bullet}\right)
$$

we find

$$
\operatorname{diripal}_{\mathrm{ev}}^{\bullet}=\operatorname{dipal}_{\mathrm{od}}^{\bullet}+\operatorname{adari}\left(\mathrm{pal}_{\mathrm{od}}^{\bullet}\right)^{-1} \cdot \text { diripal }^{\bullet} \text {. }
$$

But since $\operatorname{pal}_{\mathrm{od}}^{\bullet}=\operatorname{expari}\left(-1 / 2 \mathrm{~Pa}^{\bullet}\right)$, this simplifies to

$$
\text { diripal }_{\mathrm{ev}}^{\bullet}=-\frac{1}{2} \mathrm{~Pa}^{\bullet}+(\exp \mathcal{P}) \text {. diripal }{ }^{\bullet}
$$

with diripal ${ }^{\bullet}$ as in (236) and with the ordinary exponential $\exp \mathcal{P}$ of the elementary operator $\mathcal{P}:$

$$
\mathcal{P} . \mathrm{M}^{\bullet}:=\frac{1}{2} \operatorname{ari}\left(\mathrm{Pa}^{\bullet}, M^{\bullet}\right) \quad\left(\forall M^{\bullet} \in \mathrm{BIMU}\right) .
$$

Being the gari-dilator of a symmetral bimould, diripal $l_{\mathrm{ev}}$ is of course alternal. And since we have shown that pal $_{\mathrm{ev}}^{\bullet}$ and therefore ripal $_{\mathrm{ev}}^{\bullet}$ are "even" (i.e., pari-invariant), the same applies for diripal $_{\mathrm{ev}}^{\bullet}$, so that, as explained in Section 2 (see (89) and (90) ) the relation between diripal $^{\bullet}$ and diripal $_{\mathrm{ev}}^{\bullet}$ may be rewritten as

$$
\text { diripal }_{\mathrm{ev}}^{\bullet}=(\cosh \mathcal{P})^{-1} \cdot \frac{1}{2}(\mathrm{id}+\text { pari }) \cdot \text { diripal }{ }^{\bullet}
$$

which, appearances notwithstanding, is actually simpler than (278), as it involves only evenlength components.

In a sense, this is all we need to know. But in order to get the extra information of formula (154) or rather, in our polar specialization, the explicit expansion of diripal $_{\mathrm{ev}}^{\bullet}$ in terms of the remarkable alternals $k a_{2 r}^{\bullet}$ (polar-specialized from the $\mathfrak{k}_{2 r}^{\bullet}$ of Section 2), we must work harder. Rather than derive the expansion of diripal $_{\mathrm{ev}}^{\bullet}$ directly $^{38}$ from that of diripal ${ }^{\bullet}$ via (278) or (280), it is more convenient to reproduce the approach of (245) and (246), i.e., to set

$$
\operatorname{kasra}_{r}^{\bullet}:=(\exp \mathcal{P}) \cdot \operatorname{ganit}\left(\operatorname{nipac}^{\bullet}\right) \cdot s r a_{r}^{\bullet}=\sum_{r \leq r_{*}} \operatorname{kasra}_{r, r_{*}} \quad\left(\operatorname{kasra}_{r, r_{*}} \in B I M U_{\mathrm{r}_{*}}\right)
$$

and then regroup the (highly lacunary) components of $r_{*}$ :

$$
\sum_{1 \leq r \leq r_{*}} \frac{1}{r .(r+1)} \operatorname{kasra}_{r, r_{*}}^{\bullet} \equiv \frac{2^{1-r_{*}}}{\left(r_{*}-1\right) \cdot\left(r_{*}+1\right)} \mathrm{ka}_{r_{*}}^{\bullet} .
$$

\footnotetext{
${ }^{38}$ The direct method yields only partial but valuable information. Thus, denoting $\operatorname{Proj} \cdot M^{\bullet}$ the first coefficient of $M^{\bullet}$ in the standard eupolar basis, we may establish the identity $\operatorname{Proj}_{1} \cdot \mathcal{P}^{2 r_{*}-r} \cdot$ diripal $_{r}^{\bullet}=$ $\frac{(-2)^{r-2 r_{*}}}{r . r+1} \frac{\left(2 r_{*}-2\right) !}{(r-2) !}$ which leads to $\operatorname{Proj}_{1}$. diripal $l_{\mathrm{ev}, 2 r_{*}}^{\bullet}=\frac{2^{1-2 r_{*}}}{\left(2 r_{*}-1\right)\left(2 r_{*}+1\right)}$ which in turn yields the important normalization property $\operatorname{Proj}_{1} \cdot k a_{2 r_{*}}^{\bullet}=1$.
} 
Comparing the components $\operatorname{kasra}_{r, r_{*}}^{\bullet}$ with the earlier hasra $a_{r, r_{*}}^{\bullet}$ of (245), one even gets to understand (however dimly) why the relevant tree-combinatorial object for calculating the bimould projections in the standard basis $\left\{\mathfrak{e}_{\boldsymbol{t}}^{\bullet}\right\}$ is $\operatorname{slant}(\boldsymbol{t})$ in the case of $h a_{r}^{\bullet}$ and $\operatorname{stack}(\boldsymbol{t})$ in the case of $k a_{2 r}^{\bullet}$. Still, the calculations are quite lengthy and the whole approach leaves much to be desired. In particular, one would appreciate a more conceptual explanation for the puzzling slant/stack dichotomy.

\subsection{Characterization of $\mathrm{pal}^{\bullet} / \mathrm{pil}^{\bullet}$}

The explicit expansion of pal as given in (300) below (as a direct consequence of (122) and (123)) makes it clear that pal ${ }^{\bullet}$, and therefore pil $^{\bullet}$ too, possess exactly the pole pattern described in Proposition 3.2. To prove the converse, namely that no other Pi-polar bisymmetral varpil $^{\bullet}$ can display the same pole pattern, we must use the results of Section 8 about the standard factorization of bisymmetrals. In the case when varpil $_{1}^{\bullet}=0$, we have

$$
\text { varpil }^{\bullet}=\text { expari.bir }{ }^{\bullet} \quad \text { with } \text { bir }^{\bullet} \in \text { bialternal } .
$$

In the case when our first component varpil $l_{1}^{\bullet}$ is $\neq 1$, it is necessarily of the form $c \mathrm{Pi}^{\bullet}$ and, modulo an elementary dilation varpil $_{r}^{\bullet} \mapsto \gamma^{r}$ varpil $_{r}^{\bullet}$, we may assume $c=-1 / 2$ and get varpil $_{1}^{\bullet}$ and pil $l_{1}^{\bullet}$ to coincide, thus ensuring (according to Section 8) the existence of a factorization

$$
\text { varpil }^{\bullet}=\text { gari }\left(\text { pil }{ }^{\bullet}, \text { expari.bir } \bullet^{\bullet} \quad \text { with } \quad \text { bir }^{\bullet} \in \text { bialternal } .\right.
$$

The thing now is to focus on the first nonzero component $\operatorname{bir}_{2 r}^{\bullet}(2 r \geq 4)$. It is bound to occur linearily in the expansion of varpil $^{\bullet}$, whether the latter is of type (282) or (283). Now, bir $_{2 r}^{\bullet}$ cannot be of the form $c r i{ }_{2 r}$, which is simply alternal, not bialternal. But of all alternals, let alone bialternals, $r i_{2 r}^{\bullet}$ alone possesses precisely the pole structure described in Proposition 3.2 for pil $^{\bullet}$. This clinches the argument.

\section{Polar Bisymmetrals: Explicit Expansions}

\subsection{Explicit Expansions for pil $^{\bullet}$ and pil $_{\mathrm{ev}}^{\bullet}$}

From the $\left\{r i_{r}^{\bullet}\right\}$-expansions of pil $^{\bullet}$ 's dilator dipil ${ }^{\bullet}$ and infinitesimal generator lipil $^{\bullet}:=$ logari.pil ${ }^{\bullet}$ :

$$
\begin{array}{rlrl}
\text { dipil }^{\bullet} & =\sum_{1 \leq r} \tau_{r} \mathrm{ri}_{r}^{\bullet} & \text { with } \quad \tau_{r}=-\frac{1}{(r+1) !}, \\
\text { lipil }^{\bullet} & =\sum_{1 \leq r} \theta_{r} \mathrm{ri}_{r}^{\bullet} & \text { with } & \theta_{r}=\text { horrible, }
\end{array}
$$

we at once derive (see (39) and (478)) two equally valid expansions for pil ${ }^{\bullet}$ itself, which in their first raw form read

$$
\begin{aligned}
& \text { pil }^{\bullet}=1^{\bullet}+\sum_{r_{1}, \ldots, r_{s} \geq 1}^{s \geq 1} \tau_{r_{1}} \ldots \tau_{r_{s}} \text { Paj }^{r_{1}, \ldots, r_{s}} \underset{\text { preari }}{\longrightarrow}\left(\mathrm{ri}_{r_{1}}^{\bullet}, \ldots, \mathrm{ri}_{r_{s}}^{\bullet}\right), \\
& \text { pil }^{\bullet}=1^{\bullet}+\sum_{r_{1}, \ldots, r_{s} \geq 1}^{s \geq 1} \frac{1}{s !} \theta_{r_{1}} \ldots \theta_{r_{s}} \quad \stackrel{\text { preari }}{\longrightarrow}\left(\mathrm{ri}_{r_{1}}^{\bullet}, \ldots, \mathrm{ri}_{r_{s}}^{\bullet}\right)
\end{aligned}
$$


The main difference lies of course in the transparency of the $\tau_{r}$ 's compared with the complexity of the $\theta_{r}$ 's. But quite apart from the nature of their coefficients, the above expansions are unsatisfactory on two further counts: they are non-unique ${ }^{39}$ and involve multiple preLie brackets, which are complex, inflected expressions. So we must hasten to replace them by unique expansions involving simple, uninflected $m u$-products. There are three ways of doing this, based on the elementary series $\left\{m i_{r}^{\bullet}\right\},\left\{n i_{r}^{\bullet}\right\},\left\{r i_{r}^{\bullet}\right\}$ inductively defined as follows:

$$
\begin{array}{rll}
\mathrm{mi}_{1}^{\bullet}:=\mathrm{Pi}^{\bullet} & ; & \mathrm{mi}_{\mathrm{r}}^{\bullet}:=\operatorname{amit}\left(\mathrm{mi}_{\mathrm{r}-1}^{\bullet}\right) \cdot \mathrm{Pi}^{\bullet}, \\
\mathrm{ni}_{1}^{\bullet}:=\mathrm{Pi}^{\bullet} \quad & ; & \mathrm{ni}_{\mathrm{r}}^{\bullet}:=\operatorname{anit}\left(\mathrm{ni}_{\mathrm{r}-1}^{\bullet}\right) \cdot \mathrm{Pi}^{\bullet}, \\
\mathrm{ri}_{1}^{\bullet}:=\mathrm{Pi}^{\bullet} \quad & ; & \mathrm{ri}_{\mathrm{r}}^{\bullet}:=\operatorname{arit}\left(\mathrm{ri}_{\mathrm{r}-1}^{\bullet}\right) \cdot \mathrm{Pi}^{\bullet}
\end{array}
$$

and behaving as follows under the anti-action arit:

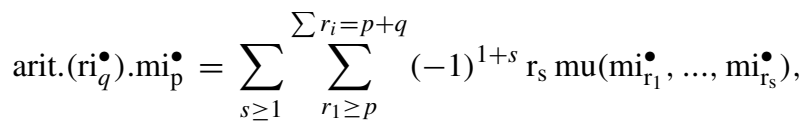

$$
\begin{aligned}
& \operatorname{arit.}\left(\mathrm{ri}_{q}^{\bullet}\right) . \mathrm{ni}_{p}^{\bullet}=\sum_{s \geq 1} \sum_{r_{s} \geq p}^{\sum r_{i}=p+q}(-1)^{1+s+q} r_{1} \mathrm{mu}^{\bullet}\left(\mathrm{ni}_{\mathbf{r}_{1}}^{\bullet}, \ldots, \mathrm{ni}_{r_{s}}^{\bullet}\right) \text {, } \\
& \text { arit. }\left(\mathrm{ri}_{q}^{\bullet}\right) \cdot \mathrm{ri}_{p}^{\bullet}=\text { p.ri } \dot{\mathrm{p}}_{\mathrm{p}+\mathrm{q}}^{\bullet}+\sum_{k \leq q} \mathrm{lu}\left(\mathrm{ri}_{k}^{\bullet}, \mathrm{ri}_{\mathrm{p}+\mathrm{q}-\mathrm{k}}^{\bullet}\right) \text {. }
\end{aligned}
$$

For $s \geq 1$ and $r_{1}+\ldots+r_{s}=r$ each of the three sets

$$
\left\{\mathrm{mu}\left(\mathrm{mi}_{\mathrm{r}_{1}}^{\bullet}, \ldots, \mathrm{mi}_{\mathrm{r}_{\mathrm{s}}}^{\bullet}\right)\right\} ;\left\{\mathrm{mu}^{\bullet}\left(\mathrm{ni}_{\mathrm{r}_{1}}^{\bullet}, \ldots, \mathrm{ni}_{\mathrm{r}_{\mathrm{s}}}^{\bullet}\right)\right\} \quad ; \quad\left\{\mathrm{mu}\left(\mathrm{ri}_{\mathrm{r}_{1}}^{\bullet}, \ldots, \mathrm{ri}_{\mathrm{r}_{\mathrm{s}}}^{\bullet}\right)\right\}
$$

consists of linearly independent bimoulds that span one and the same subspace Flexin $_{\mathrm{r}}(\mathrm{Pi})$ of Flex $(\mathrm{Pi})$. The six conversion rules between the three bases are mentioned in [4] Section 4.1. Let us recall the most useful

$$
\begin{aligned}
\mathrm{ri}_{r_{0}}^{\bullet} & \left.=\sum_{1 \leq s} \sum_{\sum r_{i}=r_{0}}(-1)^{s+1} r_{s} \operatorname{mu}_{\left(\mathrm{mi}_{\mathrm{r}_{1}}, \ldots, \mathrm{mi}_{\mathrm{r}_{1}}\right),}\right) \\
\mathrm{ri}_{r_{0}}^{\bullet} & =\sum_{1 \leq s} \sum_{\sum r_{i}=r_{0}}(-1)^{s+r} r_{1} \operatorname{mu}\left(\mathrm{ni}_{\mathrm{r}_{1}}^{\bullet}, \ldots, n i_{r_{1}}^{\bullet}\right) .
\end{aligned}
$$

The first two bases (294) of Flexin $(P i)$ have the advantage of consisting of "atoms" (simple strings of inflected units $P i$ ). The ingredients $r i_{r}^{\bullet}$ of the third basis are not atomic (it takes at least $r+1$ strings to express them) but they make up for it by being alternal.

Now, the above derivation rules (291), (292), (293) together with the two conversion rules (295), (296) make it easy ${ }^{40}$ to expand the multiple preari-brackets of (284), (285) in each of the three bases (294). In the event we get three alternative expressions

\footnotetext{
${ }^{39}$ Thus we have (286) side by side with (287), all due to the many a priori relations between multiple pre-Lie brackets.

${ }^{40}$ Since $\operatorname{preari}\left(A^{\bullet}, B^{\bullet}\right)=\operatorname{arit}\left(B^{\bullet}\right) \cdot A^{\bullet}+m u\left(A^{\bullet}, B^{\bullet}\right)$.
} 


$$
\begin{aligned}
\text { pil }^{\bullet} & =1^{\bullet}+\sum_{r_{1}, \ldots, r_{s} \geq 1}^{s \geq 1} \operatorname{Mip}^{r_{1}, \ldots, r_{s}} \operatorname{mu}\left(\mathrm{mi}_{r_{1}}^{\bullet}, \ldots, \mathrm{mi}_{r_{s}}^{\bullet}\right), \\
\mathrm{pil}^{\bullet} & =1^{\bullet}+\sum_{r_{1}, \ldots, r_{s} \geq 1}^{s \geq 1} \mathrm{Nip}^{r_{1}, \ldots, r_{s}} \operatorname{mu}\left(\mathrm{ni}_{r_{1}}^{\bullet}, \ldots, \mathrm{ni}_{r_{s}}^{\bullet}\right), \\
\text { pil }^{\bullet} & =1^{\bullet}+\sum_{r_{1}, \ldots, r_{s} \geq 1}^{s \geq 1} \operatorname{Rip}^{r_{1}, \ldots, r_{s}} \operatorname{mu}\left(\mathrm{ri}_{r_{1}}^{\bullet}, \ldots, \mathrm{ri}_{r_{s}}^{\bullet}\right) .
\end{aligned}
$$

with three rational-valued moulds $\operatorname{Mip}^{\bullet}, \mathrm{Nip}^{\bullet}, \mathrm{Rip}^{\bullet}$ defined by simple induction rules (see the next paragraph) that dually reflect the rules (288), (289), (290). In accordance with the nature of the three bases (294), Mip ${ }^{\bullet}$ and Nip $^{\bullet}$ are symmetrel while Rip ${ }^{\bullet}$ is symmetral.

The procedure for expanding $p i l_{\mathrm{ev}}^{\bullet}$ is entirely similar: one needs only retain the sole even terms $\tau_{2 r} r i_{2 r}^{\bullet}$ in (284).

\subsection{General Inductions for the Moulds $\operatorname{Mip}^{\bullet}, \mathrm{Nip}^{\bullet}, \mathrm{Rip}^{\bullet}$}

The first induction goes like this

$$
\begin{aligned}
& \operatorname{Mip}^{\emptyset}:=1, \operatorname{Mip}^{1}:=\alpha_{1}, \\
& \operatorname{Mip}^{n_{1}}:=\frac{1}{n_{1}} \operatorname{Mi}_{*}^{n_{1}}+\frac{1}{n_{1}} \sum_{0<n_{0}<n_{1}} \operatorname{Mip}^{n_{0}} \operatorname{Mi}_{n_{0}}^{n_{1}}, \\
& \operatorname{Mip}^{\boldsymbol{n}}:=\frac{1}{|\boldsymbol{n}|} \sum_{\boldsymbol{n}^{1} \cdot \boldsymbol{n}^{2}=\boldsymbol{n}} \operatorname{Mip}^{\boldsymbol{n}^{1}} \mathrm{Mi}_{*}^{\boldsymbol{n}^{2}}+\frac{1}{|\boldsymbol{n}|} \sum_{\boldsymbol{n}^{1} \cdot \boldsymbol{n}^{2} \cdot \boldsymbol{n}^{3}=\boldsymbol{n}}^{0<n_{0} \leq \operatorname{first}\left(\boldsymbol{n}^{2}\right)} \operatorname{Mip}^{\boldsymbol{n}^{1}, n_{0}, \boldsymbol{n}^{3}} \mathrm{Mi}_{n_{0}}^{\boldsymbol{n}^{2}},
\end{aligned}
$$

with

$$
\begin{aligned}
& \mathrm{Mi}_{*}^{n_{1}, \ldots, n_{r}}:=(-1)^{1+r} n_{r} \alpha_{|\boldsymbol{n}|}, \\
& \mathrm{Mi}_{n_{0}}^{n_{1}, \ldots, n_{r}}:=(-1)^{1+r} n_{r} \alpha_{|\boldsymbol{n}|-n_{0}} \text { if } 0<n_{0} \leq n_{1} \quad(:=0 \text { otherwise }) .
\end{aligned}
$$

The second induction is essentially the same under the left-right exchange

$$
\begin{aligned}
& \operatorname{Nip}^{\emptyset}:=1, \mathbf{N i p}^{1}:=\alpha_{1}, \\
& \operatorname{Nip}^{n_{1}}:=\frac{1}{n_{1}} \mathrm{Ni}_{*}^{n_{1}}+\frac{1}{n_{1}} \sum_{0<n_{0}<n_{1}} \operatorname{Nip}^{n_{0}} \mathrm{Ni}_{n_{0}}^{n_{1}}, \\
& \operatorname{Nip}^{\boldsymbol{n}}:=\frac{1}{|\boldsymbol{n}|} \sum_{\boldsymbol{n}^{1} \cdot \boldsymbol{n}^{2}=\boldsymbol{n}} \mathbf{N i p}^{\boldsymbol{n}^{1}} \mathrm{Ni}_{*}^{\boldsymbol{n}^{2}}+\frac{1}{|\boldsymbol{n}|} \sum_{\boldsymbol{n}^{1} \cdot \boldsymbol{n}^{2} \cdot \boldsymbol{n}^{3}=\boldsymbol{n}}^{0<n_{0} \leq \operatorname{last}\left(\boldsymbol{n}^{2}\right)} \mathrm{Nip}^{\boldsymbol{n}^{1}, n_{0}, \boldsymbol{n}^{3}} \mathrm{Ni}_{n_{0}}^{\boldsymbol{n}^{2}},
\end{aligned}
$$

with

$$
\begin{aligned}
& \mathrm{Ni}_{*}^{n_{1}, \ldots, n_{r}}:=(-1)^{r+|\boldsymbol{n}|} n_{1} \alpha_{|\boldsymbol{n}|}, \\
& \mathrm{Ni}_{n_{0}}^{n_{1}, \ldots, n_{r}}:=(-1)^{1+r+|\boldsymbol{n}|-n_{0}} n_{1} \alpha_{|\boldsymbol{n}|-n_{0}} \text { if } 0<n_{0} \leq n_{r} \quad(:=0 \text { otherwise }) .
\end{aligned}
$$

The third induction involves less terms and is faster to run on a computer (see Section 18.1 infra), the reason being that here the bulk of the complexity is absorbed by the "molecular" $r i_{r}^{\bullet}$ 's that replace the "atomic" $m i_{r}^{\bullet}$ 's or $n i_{r}^{\bullet}$ 's of the earlier inductions 


$$
\begin{aligned}
& \operatorname{Rip}^{\emptyset}:=1, \operatorname{Rip}^{1}:=\alpha_{1}, \quad \operatorname{Rip}^{1, \ldots, 1}:=\frac{1}{r !}\left(\alpha_{1}\right)^{r}, \\
& \operatorname{Rip}^{n_{1}}:=\frac{1}{n_{1}} \alpha_{n_{1}}+\frac{1}{n_{1}} \sum_{0<n_{0}<n_{1}} \operatorname{Rip}^{\text {times }_{0}} \mathrm{Ri}_{n_{0}}^{n_{1}}, \\
& \operatorname{Rip}^{\boldsymbol{n}}:=\frac{1}{|\boldsymbol{n}|} \boldsymbol{\operatorname { R i p }}^{\boldsymbol{n}^{\prime}} \alpha_{n_{r}}+\frac{1}{|\boldsymbol{n}|} \sum_{\boldsymbol{n}^{1} \cdot \boldsymbol{n}^{2} \cdot \boldsymbol{n}^{3}=\boldsymbol{n}}^{0<n_{0} \mid} \mathbf{N i p}^{\boldsymbol{n}^{1}, n_{0}, \boldsymbol{n}^{3}} \mathrm{Ni}_{n_{0}}^{\boldsymbol{n}^{2}},
\end{aligned}
$$

with

$$
\begin{array}{rlrl}
\mathrm{Ri}_{n_{0}}^{n_{1}} & :=n_{0} \alpha_{n_{1}-n_{0}} \text { if } n_{0}<n_{1} \quad(:=0 \text { otherwise }), \\
\mathrm{Ri}_{n_{0}}^{n_{1}, n 2} & :=+\alpha_{n_{1}+n_{1}-n_{0}} & \text { if } n_{1}<n_{0} \leq n_{2}, \\
& :=-\alpha_{n_{1}+n_{2}-n_{0}} \text { if } n_{2}<n_{0} \leq n_{1}, \\
& :=0 & \text { otherwise } \\
\mathrm{Ri}_{n_{0}}^{n_{1}, \ldots, n_{r}} & :=0 & \text { if } r \geq 3 .
\end{array}
$$

\subsection{Explicit Expansions for $p a l^{\bullet}, \mathrm{pal}_{\mathrm{ev}}^{\bullet}$ and $p a l_{\mathrm{evv}}^{\bullet}$}

We start from the $m u$-dilators dupal $^{\bullet}$, dupal $_{\mathrm{ev}}^{\bullet}$, dupal $_{\mathrm{evv}}^{\bullet}$ as described in Section 3. Applying the rule (39) we immediately derive these three expansions

$$
\begin{aligned}
& \mathrm{pal}^{\bullet}=1^{\bullet}+\sum_{\boldsymbol{w}^{1} \ldots \boldsymbol{w}^{s}=\bullet}^{r_{i} \text { even or } 1} \alpha_{r_{1}} \ldots \alpha_{r_{s}} \operatorname{Paj}^{\left|\boldsymbol{u}^{1}\right|, \ldots,\left|\boldsymbol{u}^{s}\right|} \operatorname{mu}\left(\operatorname{lan}_{\mathrm{r}_{1}}^{\bullet}, \ldots, \operatorname{lan}_{\mathrm{r}_{\mathrm{s}}}^{\bullet} x\right), \\
& \mathrm{pal}_{\mathrm{ev}}^{\bullet}=1^{\bullet}+\sum_{\boldsymbol{w}^{1} \ldots \boldsymbol{w}^{s}=\bullet}^{r_{i} \text { even }} \alpha_{r_{1} \ldots} \ldots \alpha_{r_{s}} \mathrm{Paj}^{\left|\boldsymbol{u}^{1}\right|, \ldots,\left|\boldsymbol{u}^{s}\right|} \operatorname{mu}\left(\operatorname{lan}_{\mathrm{r}_{1}}^{\bullet}, \ldots, \operatorname{lan}_{\mathrm{r}_{\mathrm{s}}}^{\bullet}\right), \\
& \mathrm{pal}_{\mathrm{evv}}^{\bullet}=1^{\bullet}+\sum_{\boldsymbol{w}^{1} \ldots \boldsymbol{w}^{s}=\bullet}^{r_{i} \text { even }} \beta_{r_{1} \ldots} \ldots \beta_{r_{s}} \operatorname{Paj}^{\left|\boldsymbol{u}^{1}\right|, \ldots,\left|\boldsymbol{u}^{s}\right|} \operatorname{mu}\left(\operatorname{lan}_{\mathrm{r}_{1}}^{\bullet}, \ldots, \operatorname{lan}_{\mathrm{r}_{\mathrm{s}}}^{\bullet}\right),
\end{aligned}
$$

with $r_{i}=r\left(\boldsymbol{w}^{i}\right)=r\left(\boldsymbol{u}^{i}\right)$; with the selfsame Bernoulli-like numbers $\alpha_{r}, \beta_{r}$ as in (121), (159); and with

$$
\operatorname{lan}_{\mathrm{r}}^{\bullet}:=\operatorname{lu}(\mathrm{I}^{\bullet}, \overbrace{\mathrm{Pa}^{\bullet}, \ldots, \mathrm{Pa}^{\bullet}}^{(r-1) \text { times }}) .
$$

The last two expansions must be preferred to the first, since they involve only even terms. Of these two even expansions, (302) is again preferrable to (301), since the passage from $\mathrm{pal}_{\mathrm{evv}}^{\bullet}$ to $\mathrm{pal} \mathbf{l}^{\bullet}$ (mu-multiplication) is so much simpler than the passage from pal $\bullet_{\mathrm{ev}}^{\bullet}$ to $\mathrm{pal} \mathbf{l}^{\bullet}$ (gari-multiplication).

But there is still room for improvement. Indeed, (302) is blighted by some redundancy since the summands on the right-hand side are not linearly independent. ${ }^{41}$ To get a true basis, we must introduce bimoulds $\operatorname{Lan}_{\epsilon_{1}, \ldots, \epsilon_{s}}^{\bullet} \in$ Flex $_{2 s}(\mathrm{~Pa})$ inductively defined by

\footnotetext{
${ }^{41}$ The products $m u\left(\operatorname{lan}_{r_{1}}^{\bullet}, \ldots, \operatorname{lan}_{r_{s}}^{\bullet}\right)$ are of course linearly independent, but cease to be so when "precomposed" by $P a j^{\bullet}$ as in (300), (301), (302).
} 


$$
\begin{aligned}
& \operatorname{Lan}_{\epsilon_{1}, \ldots, \epsilon_{s}}^{w_{1}, \ldots, w_{2 s}}=\operatorname{Lan}_{\epsilon_{1}, \ldots, \epsilon_{s-1}}^{w_{1}, \ldots, w_{2 s-2}} \operatorname{Pan}_{\epsilon_{s}}^{w_{1}, \ldots, w_{2 s}} \quad \text { with } \\
& \operatorname{Pan}_{0}^{w_{1}, \ldots, w_{2 s}}:=\mathrm{P}\left(u_{2 s-1}\right) \mathrm{P}\left(u_{2 s}\right), \\
& \operatorname{Pan}_{1}^{w_{1}, \ldots, w_{2 s}}:=\mathrm{P}\left(u_{2 s-1}\right) \mathrm{P}\left(u_{1}+\ldots+u_{2 s}\right), \\
& \operatorname{Pan}_{2}^{w_{1}, \ldots, w_{2 s}}:=\mathrm{P}\left(u_{2 s}\right) \mathrm{P}\left(u_{1}+\ldots+u_{2 s}\right) .
\end{aligned}
$$

Fixing $s$ and letting each $\epsilon_{i}$ range over $\{0,1,2\}$, except for the first $\epsilon_{1}$ which is forbidden to be 0 , we get a set of bimoulds $\operatorname{Lan}_{\epsilon_{1}, \ldots, \epsilon_{s}}^{\bullet}$ that

(i) Are linearly independent,

(ii) Span the same subspace of Flex $_{2 s}(\mathrm{~Pa})$ as the $\mathrm{Paj}^{\bullet} \circ \mathrm{mu}\left(\operatorname{lan}_{r_{1}}^{\bullet}, \ldots, \operatorname{lan}_{r_{s}}^{\bullet}\right)$,

(iii) Permit to express these $P a j^{\bullet} \circ m u\left(\operatorname{lan}_{r_{1}}^{\bullet}, \ldots, \operatorname{lan}_{r_{s}}^{\bullet}\right)$ via a simple rule.

So (302) may be rewritten more economically as

$$
\operatorname{pal}_{\mathrm{evv}}^{\bullet}=1^{\bullet}+\sum_{\epsilon_{1}, \ldots, \epsilon_{s} \in\{0,1,2\}}^{s \geq 1} \operatorname{Han}^{\epsilon_{1}, \ldots, \epsilon_{s}} \operatorname{Lan}_{\epsilon_{1}, \ldots, \epsilon_{s}}^{\bullet} \quad\left(s=\frac{1}{2} r(\bullet)\right)
$$

with a rational valued mould $\mathrm{Han}^{\bullet}$ belonging to none of the classical symmetry types but nonetheless calculable by a simple induction.

From $\mathrm{pal}_{\mathrm{evv}}^{\bullet}$, we easily go to $\mathrm{pal} \mathbf{l}^{\bullet}$, through elementary $m u$-multiplication by the archelementary factor $p a l_{\text {odd }}^{\bullet}$, and from there we go to $p i l^{\bullet}$ through the equally elementary involution swap. Moreover, of all expansions currently at our disposal, this ultimate expansion (305) for $p a l_{\mathrm{evv}}^{\bullet}$ is clearly optimal, since it involves only $2.3^{r / 2-1}$ atomic summands, as compared with the $2^{r}$ summands in each of the three expansions (297), (298), (299) for pil ${ }^{\bullet}$.

Remark If in (304) we had prohibited for $\epsilon_{1}$ the value 1 , resp. 2, instead of 0 , we would still have got two valid bases $\operatorname{Lan}_{\epsilon_{1}, \ldots, \epsilon_{r}}^{\bullet}$ and two expansions of the form (303), though with changed moulds $H^{\bullet}$. There exist yet other bases with the same indexation. These multiple choices, hardly relevant in the eupolar case, acquire real significance in the eutrigonometric case ([5]) and will be discussed there.

\section{Polar Bisymmetrals: Seven Remarks}

Remark 1 Nearly complete restoration of symmetry.

The first proof presented here (in Section 4) of the bisymmetrality of pal ${ }^{\bullet} /$ pil $^{\bullet}$ is definitely shorter than the second one, which in turn is simpler than either of the two proofs sketched in [4]. As we see it, it has two further merits: it respects the symmetry between the two swappees (unlike the earlier treatments, which gave precedence to pil ${ }^{\bullet}$ and relegated pal ${ }^{\bullet}$ to the subordinate status of a derivative object) and it does so in the most satisfactory way that could be dreamt of, by linking pal ${ }^{\bullet}$ and pil ${ }^{\bullet}$ separately to the only two completely elementary alternal series that exist in Flex(E), namely $\left\{\mathfrak{L e}_{r}^{\bullet}\right\}$ and $\left\{\mathfrak{r} \mathfrak{e}_{r}^{\bullet}\right\}$.

The linkage between each swappee and its alternal series is provided by the notion of dilator, but the two dilators in question are rather different: one is geared to the uninflected $m u$-product, the other to the inflected gari-product. The two alternal series $\left\{\mathfrak{l} \mathfrak{e}_{r}^{\bullet}\right\}$ and $\left\{\mathfrak{r} \mathbf{e}_{r}^{\bullet}\right\}$ also differ, and in much the same way. We have here, we suggest, the whole essence of dimorphy in a nutshell: a symmetry that is nearly complete, yet stops just short of being 
thoroughly, dully, and barrenly complete. In fact the whole flexion structure--dimorphy's natural framework-is largely though not perfectly self-dual under swap. So is its core ARI//GARI. And so is the core's core, consisting of the two pairs $\mathrm{pal}^{\bullet} / \mathrm{pil}^{\bullet}$ and $\mathrm{tal}^{\bullet} / \mathrm{til}^{\bullet}$. Experience shows that such mathematical structures are among the most fecund.

Remark 2 Pervasiveness of parity.

Considerations of parity are paramount in all branches of the theory, not just in the factorization of the key bimoulds but also when it comes to constructing and describing their length- $r$ components.

Regarding the factorizations, they come in all sorts and shapes. Thus, all three formulae (129), (130), (131) are logically independent, carry unrelated even factors, and involve two distinct group laws, $m u$ and gari. Nor is the phenomenon restricted to the eupolar context; it extends to such objects as the important bimould $\mathrm{Zag}^{\bullet}$, though with a nuance: unlike eupolar bimoulds, which are automatically invariant under pari $\circ$ neg, general bimoulds such as $\mathrm{Zag}^{\bullet}$ react differently to pari and $n e g$, leading to a more intricate factorization pattern, with three factors $\operatorname{Zag}_{I}^{\bullet}, \operatorname{Zag}_{I I}^{\bullet}, \operatorname{Zag}_{I I I}^{\bullet}$, the first of which again splits into three subfactors. Let us recall the corresponding identities: ${ }^{42}$

$$
\begin{aligned}
& \mathrm{Zag}^{\bullet}=\operatorname{gari}\left(\mathrm{Zag}_{\mathrm{I}}^{\bullet}, \mathrm{Zag}_{\mathrm{II}}^{\bullet}, \mathrm{Zag}_{\mathrm{III}}^{\bullet}\right), \\
& \mathrm{Zag}_{\mathrm{I}}^{\bullet}=\operatorname{gari}\left(\mathrm{tal}^{\bullet}, \text { invgari.pal }{ }^{\bullet}, \text { expari.røma }{ }^{\bullet}\right) .
\end{aligned}
$$

Regarding the mould components, the even/odd dichotomy makes itself felt in this way: whereas we have to work in order to find the even-length components of our bisymmetrals ${ }^{43}$, their odd-length components immediately and effortlessly follow, and that too under any one of at least four distinct mechanisms. ${ }^{44}$ The dichotomy also holds for the components of $\mathrm{Zag}^{\bullet}$ and those of each of its three factors. Thus, constructing the even-length components of $Z_{a g_{I}^{\bullet}}$ or $Z a g_{I I}^{\bullet}$ is hard work, while the odd-length components easily follow. With $Z a g_{I I I}^{\bullet}$, it is exactly the reverse.

Ultimately, the dominance of parity in flexion theory can be traced back to one root cause: the essential parity of bialternals (see Section 7 infra). Germane considerations also explain the existence of a surperalgebra SUARI parallel to ARI (see [2], Section 24, pp. 456-459).

Remark 3 Native complexity of bisymmetrals.

No bisymmetrality proof for $\mathrm{pal}^{\bullet} / \mathrm{pil}^{\bullet}$ is entirely elementary, even though the first of the two proofs presented here (in Section 4.3) keeps complications down to a minimum. Bisymmetrality proofs for the trigonometric $t a l^{\bullet} / \mathrm{til}^{\bullet}$ are even longer and harder.

This relative difficulty in proving what is after all the signature property of our two bimould pairs (their birthmark as it were and the one reason behind their ubiquity in multizeta theory) simply reflects the non-trivial nature of these objects-their native and irreducible complexity.

\footnotetext{
${ }^{42}$ See [4], (9.1) and (9.3).

${ }^{43}$ This applies for the eutrigonometric $\mathrm{tal}^{\bullet} / \mathrm{til}^{\bullet}$ even more than for the eupolar $\mathrm{pal} \boldsymbol{\bullet}^{\bullet} / \mathrm{pil}^{\bullet}$.

${ }^{44}$ We can use either the three identities (129), (130), (131) in Section 3 or again the "secondary-to-primary" identity (4.85) in [4].
} 
Remark 4 Nature picks exactly the right polar specializations.

Though the two structures Flex $(\mathrm{Pi})$ and $\mathrm{Flex}(\mathrm{Pa})$ are strictly isomorphic, the two polar specializations, when applied to a given element of Flex $(\mathfrak{E})$, often lead to rational functions that differ widely in appearance, complexity, and (rational) degree.

Thus $\mathrm{pal}^{\bullet} / \mathrm{pil}^{\bullet}$ is far simpler than $\mathrm{par}^{\bullet} / \mathrm{pir}^{\bullet}$. Unlike $\mathrm{par}^{\bullet} / \mathrm{pir}^{\bullet}$, it admits a trigonometric counterpart. And unlike par $^{\bullet} /$ pir $^{\bullet}$, it spontaneously occurs in the double trifactorization of $\mathrm{Zag}^{\bullet} / \mathrm{Zig}^{\bullet}$.

Similarly, the alternal series $\left\{\mathfrak{r} \mathfrak{e}_{r}^{\bullet}\right\}$ is simpler when specialized to $\left\{r i_{r}^{\bullet}\right\}$ under $\mathfrak{E} \mapsto P i$ than when specialized to $\left\{r a_{r}^{\bullet}\right\}$ under $\mathfrak{E} \mapsto P a$. Conversely, the series $\left\{\mathfrak{l e}_{r}^{\bullet}\right\},\left\{\mathfrak{h} \mathfrak{e}_{r}^{\bullet}\right\},\left\{\mathfrak{k} \mathfrak{e}_{2 r}^{\bullet}\right\}$ are simpler in their incarnation as $\left\{l a_{r}^{\bullet}\right\},\left\{h a_{r}^{\bullet}\right\},\left\{k a_{2 r}^{\bullet}\right\}$ than as $\left\{l i_{r}^{\bullet}\right\},\left\{h i_{r}^{\bullet}\right\},\left\{k i_{2 r}^{\bullet}\right\}$.

Lastly, as if to complete this picture of harmony, it so happens that it is precisely in their simpler form $\left\{r i_{r}^{\bullet}\right\}$ and $\left\{l a_{r}^{\bullet}\right\},\left\{h a_{r}^{\bullet}\right\},\left\{k a_{2 r}^{\bullet}\right\}$ that the four alternals series occur in the dilators of $\mathrm{pal}^{\bullet} / \mathrm{pil}^{\bullet}$.

Remark 5 Direct vs inverse bisymmetrals.

In some ways (e.g., with regard to their separators and dilators) the gari-inverses of bisymmetrals are better-behaved than the originals. This fact, already noticeable with eupolars, becomes particularly striking in the eutrigonometric case: compare for example the transparent right-hand side of (4.88) in [4] with that of (4.87), for which no simple closed formula exists.

But the main difference is one of "universality": whereas $p a l^{\bullet} /$ pil $^{\bullet}$ and par $^{\bullet} / p^{\bullet} \boldsymbol{p}^{\bullet}$ and indeed all "intermediate" bisymmetrals ${ }^{45}$ have different gepar-separators, the separators of the gari-inverses ripal $^{\bullet} /$ ripil $^{\bullet}$ and ripar $^{\bullet} /$ ripir $^{\bullet}$ (and of all other exceptional, non neginvariant bisymmetrals) do coincide. ${ }^{46}$

Lastly, we may note that in the applications to multizeta algebra, it is the inverse polar bisymmetrals ripal $^{\bullet} /$ ripil $^{\bullet}$ and the direct trigonometric bisymmetrals tal $^{\bullet} /$ til $^{\bullet}$ that matter most.

Remark 6 Coexistence of inflected and non-inflected operations.

Quite often, when comparing flexion formulae, ${ }^{47}$ one is struck by a recurrent anomaly: that of complex inflected operations like gari, expari, etc. inexplicably morphing into noninflected ones like $т и$, ехрти etc. While there is no neat, sweeping reason for this stealthy tendency towards "desinflexion," but only case to case explanations, one may still point to the existence of a large ideal $A R I_{\text {intern }}$ of $A R I$ and of a large normal subgroup GARI intern of GARI where ari and gari reduce to $l u$ and $m u$ (but with the order of the arguments reversed). See Section 1.11 supra.

Remark 7 The trigonometric bisymmetral $\operatorname{tal}^{\bullet} / \mathrm{til}^{\bullet}$.

\footnotetext{
${ }^{45}$ Of type $\operatorname{gari}\left(\right.$ pal $l^{\bullet}$, expari $\left(\right.$ bal $\left.\left.l^{\bullet}\right)\right)$ with bal ${ }^{\bullet}$ any bialternal.

${ }^{46}$ This is not always an asset: it is sometimes useful to have simple criteria that tell the canonical from the non-canonical bisymmetrals.

${ }^{47}$ For example (247), (248), (249).
} 
The 'trigonometric specialization'

$$
(\mathfrak{E}, \mathfrak{O}) \mapsto\left(\mathrm{Qi}_{c}, \mathrm{Qa}_{c}\right) \quad \text { with } \quad \mathrm{Qi}_{c}^{w_{1}}:=\frac{c}{\tan \left(c v_{1}\right)} ; \mathrm{Qa}_{c}^{w_{1}}:=\frac{c}{\tan \left(c u_{1}\right)}
$$

is no proper specialization, since $\mathrm{Qi}_{c}^{\bullet}$ and $\mathrm{Qa}_{c}^{\bullet}$ are only approximate units, due to the corrective terms $\pm c^{2}$ in the identities (3.28) and (3.29) of [4]. See also Section 17.12 infra. One should therefore be prepared for serious complications when going from $\mathrm{pal}^{\bullet} / \mathrm{pil}^{\bullet}$ to the trigonometric equivalent $t a l^{\bullet} / \mathrm{til}^{\bullet}$, and in that respect the trigonometric bisymmetrals do not disappoint. A long monograph [6] will be devoted to them and their natural environment, the structures Flex $\left(Q i_{c}\right)$ and Flex $\left(Q a_{c}\right)$, which are not isomorphic to the polar prototypes nor indeed to each other.

We shall be content here with a few hints, to highlight the key steps in the transition from eupolar to eutrigometric. The formula (113) linking pil $^{\bullet}$ to its gari-dilator dipil ${ }^{\bullet}$ survives unchanged (as to its general form). The link between pal ${ }^{\bullet}$ to its $m u$-dilator $d u p a l^{\bullet}$ also survives, especially regarding the even factors, though not exactly in the "differential" form (119) but rather in the "integral" form (300), with the auxiliary mould $P a j \bullet$ replaced, unsurprisingly, by a more complex $T a j^{\bullet}$. But the main change is this: while the polar dilators had their components dipil $_{r}^{\bullet}$ resp. dupal $l_{r}^{\bullet}$ simply proportional to $r_{r}^{\bullet}$, resp. $l a_{r}^{\bullet}$ (or rather $l a n_{r}^{\bullet}$ ), the trigonmetric dilator components ditil $_{r}^{\bullet}$ and dutal $_{r}^{\bullet}$ take their values in two $\delta(r)$-dimensional spaces of alternals, with a fast (faster than polynomially) increasing $\delta(r)$. So now at each (even) step, we have to determine not one, but $\delta(r)$ rational coefficients on both sides, and to understand the affine (or linear, modulo the "earlier" coefficients) correspondance between the two sets. The alternal series $\left\{h a_{r}\right\}$ and $\left\{k a_{2 r}\right\}$ also survive (with single components morphing into linear spaces) and so does their connection with the even factors of the inverse bisymmetrals. Altogether, although almost every single statement of Section 3 has its counterpart in the new setting, we experience a steep increase in difficulty, resulting in an even more diverse and interesting situation.

\section{Essential Parity of Bialternals}

This section is devoted to establishing the decomposition ${ }^{48}$

$$
\mathrm{ARI}^{\mathrm{a} l / \mathrm{al}}=\mathrm{ARI}^{\dot{a} \mathrm{l} / \dot{a} \mathrm{l}} \oplus \mathrm{ARI}^{\mathrm{a} \mathrm{l} / \underline{\mathrm{al}}}
$$

of the space $A R I^{a l / a l}$ of all bialternals into:

(i) A large, regular part $A R I^{\underline{a l}} / \underline{a l}$, consisting of even bimoulds and stable under the aribracket,

(ii) A small, exceptional part $A R I^{\dot{a} l / \dot{a} l}:=B I M U_{1}^{\text {odd }}$, consisting of $o d d$ bimoulds of length one and endowed with a bilinear mapping oddari into $A R I^{a l} / \underline{a l}$.

Everything rests on the following statement.

Proposition 7.1 (Parity of bialternals) Any nonzero bialternal bimould $A^{\bullet}$ purely of length $r>1$ is neg-invariant or, if you prefer, an even function of its double index sequence: $A^{w} \equiv A^{-w}$.

\footnotetext{
${ }^{48}$ See [4] Section 2.7.
} 
Proof Alternality implies invariance under mantar $:=-$ anti.pari. Bialternality, therefore, implies invariance under neg.push, with

$$
\begin{aligned}
\text { neg.push }: & =\text { mantar.swap.mantar.swap } \\
& =\text { anti.swap.anti.swap. }
\end{aligned}
$$

The push operator, we recall, is idempotent of order $r+1$ when acting on $B I M U_{r}$, i.e., on bimoulds of length $r$.

Let us assume that $A^{w}$ is odd in $\boldsymbol{w}$, and show that this implies $A^{\boldsymbol{w}} \equiv 0$.

For an even length $r$, this follows at once from the neg.push-invariance:

$$
A^{w}=(\text { neg.push })^{r+1} \cdot A^{w}=\operatorname{neg}^{r+1} \cdot \text { push }^{r+1} \cdot A^{w}=\text { neg. } A^{w}=-A^{w} .
$$

For an odd length, the argument is more roundabout. Note first that for $A^{w}$, which we assumed to be odd in $w$, invariance under neg.push amounts to invariance under -push. Here again, it turns out that the absence of non-trivial solution does not require the full bialternality of $A^{\bullet}$, but only its alternality and invariance under -push. So let us prove this stronger statement:

Lemma 7.1 (Alternality and push-invariance) No nonzero bimould $A^{\bullet}$ purely of length $r>$ 1 can be simultaneously alternal and invariant under - push.

Proof Here again, the statement is obvious for $r$ even. So let us consider an odd length of the form $r=2 t+1 \geq 3$.

Since we shall subject $A^{w}$ to two linear operators, pus and push, respectively of order $r$ and $r+1$ when restricted to $B I M U_{r}$, and since pus (resp. push) reduces to a circular permutation in the "short" (resp. "long") bimould notation, we shall make use of both. Let us recall the conversion rule

$$
A^{\left[w_{0}^{*}\right], w_{1}^{*}, \ldots, w_{r}^{*}}(\text { long }) \longleftrightarrow A^{w_{1}, \ldots, w_{r}} \quad(\text { short })
$$

with the dual conditions on upper and lower indices

$$
\begin{aligned}
& u_{0}^{*}=-\left(u_{1}+\ldots u_{r}\right), \quad u_{i}^{*}=u_{i} \forall i \geq 1, \\
& v_{0}^{*} \quad \operatorname{arbitrary}, v_{i}^{*}-v_{0}^{*}=v_{j} \forall i \geq 1 .
\end{aligned}
$$

To show that $A^{\bullet}=0$, we start with the elementary alternality relation:

$$
0=\sum_{\boldsymbol{w} \in \operatorname{sha}\left(\boldsymbol{w}^{\prime}, \boldsymbol{w}^{\prime \prime}\right)} A^{\boldsymbol{w}} \quad \text { with } \boldsymbol{w}^{\prime}=\left(w_{1}, \ldots, w_{2 t}\right) \text { and } \boldsymbol{w}^{\prime \prime}=\left(w_{2 t+1}\right)
$$

which reads

$$
0=\sum_{1 \leq j \leq 2 t+1} A^{\overline{w_{1}, \ldots, w_{j-1}}, w_{2 t+1}, \overline{w_{j}, \ldots, w_{2 t}}} .
$$

Due to the invariance of $A^{\bullet}$ under - push, this may be rewritten as

$$
0=\sum_{1 \leq j \leq 2 t+1}(-1)^{j}\left(\operatorname{push}^{\mathrm{j}} . A\right)^{\overline{w_{1}, \ldots, w_{j-1}}, w_{2 t+1}, \overline{w_{j}, \ldots, w_{2 t}}} .
$$

In the "long" notation (of greater relevance here), this becomes

$$
\begin{aligned}
0 & =\sum_{1 \leq j \leq 2 t+1}(-1)^{j}\left(\operatorname{push}^{\mathrm{j}} . A\right)^{\left[w_{0}\right], \overline{w_{1}, \ldots, w_{j-1}}, w_{2 t+1}, \overline{w_{j}, \ldots, w_{2 t}}} \\
& =\sum_{1 \leq j \leq 2 t+1}(-1)^{j} A^{\left[w_{2 t+1}\right], \overline{w_{j}, \ldots, w_{2 t}}, w_{0}, \overline{w_{1}, \ldots, w_{j-1}}} .
\end{aligned}
$$


Under the exchange $w_{0} \leftrightarrow w_{2 t+1}$, the last identity becomes

$$
\begin{aligned}
0 & =\sum_{1 \leq j \leq 2 t+1}(-1)^{j} A^{\left[w_{0}\right], \overline{w_{j}, \ldots, w_{2 t}}, w_{2 t+1}, \overline{w_{1}, \ldots, w_{j-1}}} \\
& =\sum_{1 \leq j \leq 2 t+1}(-1)^{j} A^{\left[w_{0}\right], \overline{w_{j}, \ldots, w_{2 t+1}}, \overline{w_{1}, \ldots, w_{j-1}}} .
\end{aligned}
$$

Or again, reverting to the short notation,

$$
0=\sum_{1 \leq j \leq 2 t+1}(-1)^{j} A^{\overline{w_{j}, \ldots, w_{2 t+1}}, \overline{w_{1}, \ldots, w_{j-1}}} .
$$

On the other hand, alternality implies pus-neutrality ${ }^{49} \sum p u s^{j} A^{\bullet} \equiv 0$, which reads

$$
0=\sum_{1 \leq j \leq 2 t+1} A^{\overline{w_{j}, \ldots, w_{2 t+1}}, \overline{w_{1}, \ldots, w_{j-1}} .}
$$

From (315) and (316), we get by addition

$$
0=\sum_{0 \leq k \leq t} A^{\overline{w_{2 k+1}, \ldots, w_{2 t+1}}, \overline{w_{1}, \ldots, w_{2 k}}}
$$

and by subtraction

$$
0=\sum_{1 \leq k \leq t} A^{\overline{w_{2 k}, \ldots, w_{2 t+1}}, \overline{w_{1}, \ldots, w_{2 k-1}}} .
$$

Under the change $\left(w_{2}, w_{3}, \ldots, w_{2 t+1}, w_{1}\right) \rightarrow\left(w_{1}, w_{2}, \ldots, w_{2 t+1}\right),(318)$ becomes

$$
0=\sum_{1 \leq k \leq t} A^{\overline{w_{2 k+1}, \ldots, w_{2 t+1}}, \overline{w_{1}, \ldots, w_{2 k}} .}
$$

Subtracting (319) from (317), we end up with $A^{w_{1}, . ., w_{r}} \equiv 0$.

\section{Standard Factorization of Bisymmetrals}

This section is devoted to establishing the factorization ${ }^{50}$ :

$$
\mathrm{GARI}^{\text {as } / \mathrm{as}}=\operatorname{gari}\left(\mathrm{GARI}^{\text {às} / \text { às }}, \mathrm{GARI}^{\mathrm{as}} / \underline{\mathrm{as}}\right)
$$

of the set $G A R I^{\text {as/as }}$ of all bisymmetrals into

(i) A large, regular factor $G A R I \underline{a s} / \underline{a s}$ consisting of even bimoulds ${ }^{51}$ and stable under the gari product,

(ii) A small, exceptional factor $G A R I^{\dot{a} s / a}$ s consisting of special bimoulds derived from so-called flexion units and with components that are alternately odd/even, i.e., invariant under pari.neg rather than neg.

The proof rests on the construction and properties of the special bisymmetrals $\mathfrak{e s s}^{\bullet}$ and $\mathfrak{o s s}^{\bullet}$ (see Proposition 3.1, supra) and on the following statement:

\footnotetext{
${ }^{49}$ See [4], Section 2.4. For a proof, see below, Section 3.

${ }^{50}$ See [4], Section 2.8 .

${ }^{51}$ They are even functions of their multiindex $\boldsymbol{w}$, but may possess non-vanishing components of any length, even or odd.
} 
Proposition 8.1 (Factorization of bisymmetrals) Any bisymmetral pair of swappees $\mathrm{Sa} \bullet / / \mathrm{Si}^{\bullet}$ simultaneously factor as

$$
\begin{aligned}
& \mathrm{Sa}^{\bullet}=\operatorname{gari}\left(\mathrm{Sal}^{\bullet}, \mathrm{Sar}^{\bullet}\right)=\operatorname{gira}\left(\mathrm{Sal}^{\bullet}, \mathrm{Sar}^{\bullet}\right), \\
& \mathrm{Si}^{\bullet}=\operatorname{gari}\left(\mathrm{Sil}^{\bullet}, \mathrm{Sir}^{\bullet}\right)=\operatorname{gira}\left(\mathrm{Sil}^{\bullet}, \mathrm{Sir}^{\bullet}\right),
\end{aligned}
$$

(i) with $\mathrm{Si}^{\bullet}=$ swap.Sa ${ }^{\bullet}, \mathrm{Sil}^{\bullet}=$ swap.Sal ${ }^{\bullet}, \mathrm{Sir}^{\bullet}=$ swap.Sar${ }^{\bullet}$,

(ii) with bisymmetral right factors that are at once neg- and gush-invariant ${ }^{52}$,

(iii) with bisymmetral left factors that are at once pari.neg- and pari.gush-invariant.

In other words,

$$
\begin{aligned}
& \operatorname{Sar}^{\bullet}, \operatorname{Sir}^{\bullet} \in G A R I_{n e g}^{\mathrm{as} / \mathrm{as}}=G A R I_{\text {gush }}^{\mathrm{as} / \mathrm{as}}=: G A R I^{\underline{a s} / \underline{a s}}, \\
& \text { Sal }^{\bullet}, \text { Sil }^{\bullet} \in G A R I_{\text {pari.neg }}^{\mathrm{as} / \mathrm{as}}=G A R I_{\text {pari.gush }}^{\mathrm{as} / \mathrm{as}} .
\end{aligned}
$$

The above decompositions are not unique, but two of them stand out, namely the one in which

$$
\mathrm{Sal}^{\bullet}=\mathfrak{e s s}^{\bullet} \quad \text { with }-\frac{1}{2} \mathfrak{E}^{w_{1}}=\mathrm{Sal}^{w_{1}}=\frac{1}{2}\left(\mathrm{Sa}^{w_{1}}-\mathrm{Sa}^{-w_{1}}\right)
$$

and the one in which

$$
\mathrm{Sil}^{\bullet}=\mathbf{o s s}^{\bullet} \quad \text { with }-\frac{1}{2} \mathfrak{O}^{w_{1}}=\operatorname{Sil}^{w_{1}}=\frac{1}{2}\left(\mathrm{Si}^{w_{1}}-\mathrm{Si}^{-w_{1}}\right) .
$$

These "co-canonical" decompositions involve two conjugate flexion units $\mathfrak{E}$ and $\mathfrak{O}$ and, though distinct, easily translate into one another under the classical relation ${ }^{53}$ between $\mathfrak{e s s}^{\bullet}$ and $\mathfrak{o s s}^{\bullet}$.

Proof It rests on the Proposition 7.1 of the preceding section, in conjunction with the two following lemmas.

Lemma 8.1 (First components of bisymmetrals) If the length-one component Sal ${ }^{w_{1}}$ of a bisymmetral bimould Sal ${ }^{\bullet}$ is an even function of $w_{1}=\left(\begin{array}{c}u_{1} \\ v_{1}\end{array}\right)$, it may be anything, but if it is an odd function, it is necessarily a flexion unit.

Proof Let $u_{0}, u_{1}, u_{2}$ be constrained by $u_{0}+u_{1}+u_{2}=0$ and let $v_{0}, v_{1}, v_{2}$ be defined up to a common additive constant. At length 2 , the unique symmetrality relation for $\mathrm{Sal}{ }^{\bullet}$ may be written as:

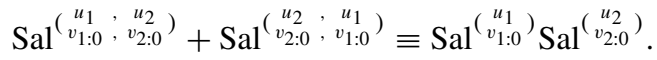

Due to $S a l^{w_{1}}$ being odd, this yields

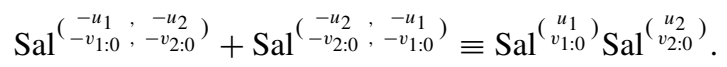

Likewise, the unique symmetrality relation for $\mathrm{Sal}^{\bullet}$ may be written as

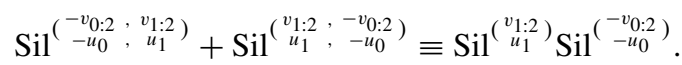

In the $u_{i}$-variables, this translates into

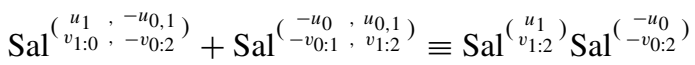

\footnotetext{
${ }^{52}$ We recall that gush $:=$ neg.gantar.swap.gantar.swap with gantar $:=$ invmu.anti.pari.

${ }^{53}$ See Section 9 infra or formula (4.63) in Section 4.2 of [4].
} 
or again, due to imparity and to $\sum u_{i}=0$ :

$$
\operatorname{Sal}^{\left(\begin{array}{c}
u_{1}, \\
v_{1: 0}, u_{2}
\end{array}, v_{2: 0}\right)}+\operatorname{Sal}^{\left(\begin{array}{c}
-u_{0} \\
-v_{0: 1},
\end{array},-v_{2: 1}\right)} \equiv-\operatorname{Sal}^{\left(\begin{array}{c}
u_{1} \\
v_{1: 2}
\end{array}\right)} \operatorname{Sal}^{\left(\begin{array}{c}
u_{0} \\
v_{0: 2}
\end{array}\right)} .
$$

Let $E_{1}$ be the identity obtained by adding the three circular permutations of (327) and (328), and $E_{2}$ the identity obtained by adding the six permutations, circular or anticircular, of (329). The left-hand sides of $E_{1}$ and $E_{2}$ clearly coincide, while their right-hand sides coincide only up to the sign. Equating these right-hand sides, we find

$$
4\left(\operatorname{Sal}^{\left({ }_{1: 0}^{u_{1}}\right)} \operatorname{Sal}^{\left(\begin{array}{c}
u_{2} \\
v_{2: 0}
\end{array}\right)}+\operatorname{Sal}^{\left(\begin{array}{c}
u_{2} \\
v_{2: 1}
\end{array}\right)} \operatorname{Sal}^{\left(\begin{array}{c}
u_{0} \\
v_{0: 1}
\end{array}\right)}+\operatorname{Sal}^{\left(v_{0: 2}^{u_{0}}\right)} \operatorname{Sal}^{\left(\begin{array}{c}
u_{1} \\
\left.v_{1: 2}\right)
\end{array}\right)}\right) \equiv 0
$$

which is precisely the symmetrical characterization of a flexion unit.

Remark 1 On the face of it, the requirement that the length-1 component to be a flexion unit is merely a necessary condition for the existence of a bisymmetral "continuation" at all lengths. However, the theory of unit-generated bisymmetrals $\mathfrak{e s s}^{\bullet}$ shows this condition to be (miraculously) sufficient. ${ }^{54}$ This is probably the best a posteriori justification for singling out this notion of flexion unit, though by no means the only one.

Remark 2 Had we assumed $\mathrm{Sal}^{\bullet}$ to be even, we would have found no constraints at all on the length-1 component-which was only to be expected, since the ari-exponential of that length- 1 component is automatically in $G A R I \underline{\text { as }}$ as .

Remark 3 One should not be too exercised over the presence of the factor 4 in (330), but rather observe that it vanishes after the change $S a l^{w_{1}}=-\frac{1}{2} \mathfrak{E}^{w_{1}}$ which, as it happens, the construction of $\mathfrak{s s}^{\bullet}$ quite naturally imposes.

Lemma 8.2 (General and even bisymmetrals) Though not a group, the set GARI ${ }^{\text {as/as }}$ of all bialternals is stable under both gari- and gira-postcomposition by the group GARI $\underline{\text { as }} \underline{\underline{\text { as }}}$ of even bisymmetrals, and the identity holds:

$$
\operatorname{gari}\left(\mathrm{S}_{1}^{\bullet}, \mathrm{S}_{2}^{\bullet}\right) \equiv \operatorname{gira}\left(\mathrm{S}_{1}^{\bullet}, \mathrm{S}_{2}^{\bullet}\right) \in \text { as } / \text { as } \quad\left(\forall \mathrm{S}_{1}^{\bullet} \in \text { as } / \text { as }, \forall \mathrm{S}_{2}^{\bullet} \in \underline{a s} / \underline{a s}\right) .
$$

Proof Here, gira stands for the pull-back of gari under the basic involution swap. Both group laws are related as follows ${ }^{55}$ :

$$
\operatorname{gira}\left(S_{1}^{\bullet}, S_{2}^{\bullet}\right)=\operatorname{ganit}\left(\operatorname{rash} . S_{2}^{\bullet}\right) \cdot \operatorname{gari}\left(S_{1}^{\bullet}, \operatorname{ras} . S_{2}^{\bullet}\right)
$$

with non-linear operators ras, rash defined by

$$
\begin{aligned}
\operatorname{ras} . S_{2}^{\bullet} & =\text { invgari.swap.invgari.swap. } S_{2}^{\bullet}, \\
\operatorname{rash} . S_{2}^{\bullet} & =\operatorname{mu}\left(\text { push.swap.invmu.swap. } S_{2}^{\bullet}, S_{2}^{\bullet}\right) .
\end{aligned}
$$

But since in Lemma 8.2 the right factor $S_{2}^{\bullet}$ is in $G A R I \underline{\text { as }} / \underline{\text { as }}$ and since gari and gira coincide on $G A R I \underline{\text { as }}$ as (even as ari and ira coincide on $A R I \underline{\text { al } / \text { al }}$ ), this implies

$$
\text { ras. } S_{2}^{\bullet}=\text { invgari.invgira. } S_{2}^{\bullet}=S_{2}^{\bullet} \text {. }
$$

\footnotetext{
${ }^{54}$ See Sections 3 and 4 supra.

${ }^{55}$ See Section 1.5 supra or [4], Section 2.3 This universal identity holds for any factors $S_{1}^{\bullet}, S_{2}^{\bullet}$.
} 
Likewise, any bimould of as/as type is automatically gush-invariant (even as any bimould of al/al type is automatically push-invariant). See [4], Section 2.4. This in turn implies

$$
\operatorname{rash} . S_{2}^{\bullet}=1^{\bullet} \quad \text { and } \operatorname{ganit}\left(\operatorname{rash} . S_{2}^{\bullet}\right)=\mathrm{id}
$$

and establishes (331).

Remark 4 Thus $S_{2}^{\bullet}$ is the only factor that really matters when comparing $\operatorname{gari}\left(S_{1}^{\bullet}, S_{2}^{\bullet}\right)$ and $\operatorname{gira}\left(S_{1}^{\bullet}, S_{2}^{\bullet}\right)$. This is less surprising than may appear at first sight, since the gari and gira products are linear in the left factor and violently non-linear in the right factor.

We can now return to the proof of Proposition 8.1. To define our left factor $S a l^{\bullet}$ we set

$$
\mathrm{Sal}_{r}^{\bullet}:=\mathfrak{e s s}^{\bullet} \quad \text { with } \quad-\frac{1}{2} \mathfrak{E}^{w_{1}}:=\frac{1}{2}\left(\mathrm{Sa}^{w_{1}}-\mathrm{Sa}^{-w_{1}}\right) .
$$

By the general theory of Sections 3 and 4 supra, this left factor is not just bisymmetral, but also invariant under pari.neg. Let us now address the construction of the right factor $\operatorname{Sar}^{\bullet}$. For each $r$, we can construct bimould pairs $\left(\mathrm{Sa}_{r}^{\bullet}, s a r_{r}^{\bullet}\right)$ by the following induction. For $r=1$ we set

$$
\begin{aligned}
\mathrm{Sa}_{1}^{\bullet} & :=\mathrm{Sa}^{\bullet}, \\
\mathrm{Sar}_{1}^{\bullet} & :=\frac{1}{2}\left(\mathrm{Sa}^{w_{1}}+\mathrm{Sa}^{-w_{1}}\right)
\end{aligned}
$$

and for $r>1$ we set

$$
\begin{aligned}
\mathrm{Sa}_{r}^{\bullet} & :=\operatorname{gari}\left(\mathrm{Sa}^{\bullet}, \operatorname{expari}\left(-\operatorname{sar}_{1}^{\bullet}\right), \ldots, \operatorname{expari}\left(-\operatorname{sar}_{r-1}^{\bullet}\right)\right), \\
\operatorname{sar}_{r}^{w_{1}, \ldots, w_{r}} & :=\operatorname{Sa}_{r}^{w_{1}, \ldots, w_{r}}-\operatorname{Sal}^{w_{1}, \ldots, w_{r}}, \\
\operatorname{sar}_{r}^{w_{1}, \ldots, w_{k}} & :=0 \quad \text { if } \quad k \neq r .
\end{aligned}
$$

Clearly

$$
\operatorname{sar}_{r}^{\bullet} \in \mathrm{BIMU}_{r} \quad \text { and } \mathrm{Sa}_{r}^{\bullet} \equiv \mathrm{Sal}^{\bullet} \bmod \oplus_{r \leq r^{\prime}} \mathrm{BIMU}_{r^{\prime}} .
$$

Let us now check that

(i) Each $S a_{k}^{\bullet}$ is in $G A R I^{\text {as/as }}$;

(ii) Each $a r_{k}^{\bullet}$ is in $A R I \underline{\text { as}} / \underline{\text { as}}$;

(iii) And therefore each expari $\left( \pm \operatorname{sar}_{k}^{\bullet}\right)$ is in $G A R I \underline{\text { as }}$ as .

This obviously holds for $k=1$. If it holds for all $k<r$, then by Lemma $2.1 S a_{k}^{\bullet}$ is also in $G A R I^{\text {as/as }}$, as the gari-product of a bimould of type as /as by a string of several bimoulds of type $\underline{a s} / \underline{a s}$. As for $s a r_{r}^{\bullet}$, it is defined as the difference of length- $r$ components of two bisymmetral bimoulds, $\mathrm{Sa}_{r}^{\bullet}$ and $\mathrm{Sal}^{\bullet}$, whose earlier components coincide. It is therefore not just of type al/al (bialternal) but also, by Lemma 7.1 in the preceding section, of type $\underline{\mathrm{al}} / \underline{\mathrm{al}}$ (bialternal and even), and its ari-exponential is automatically $\underline{a s} / \underline{a s}$.

Summing up, we arrive at a factorization of the announced type (321), with a left factor defined by (337) and a right factor defined by

$$
\operatorname{Sar}^{\bullet}=\lim _{r \rightarrow \infty} \operatorname{gari}\left(\operatorname{expari}\left(\operatorname{sar}_{r}^{\bullet}\right), \ldots, \operatorname{expari}\left(\operatorname{sar}_{1}^{\bullet}\right)\right) .
$$

The swappee factorizations (322) immediately follow, again under (332). 


\section{Polar Bialternals: First Main Source}

After our in-depth study of the central but exceptional (i.e., non neg-invariant) bisymmetrals, we can now turn to our first instance of regular (i.e., neg-invariant) bisymmetrals, and thence to the corresponding (automatically regular) bialternals.

Applying the general results of Proposition 8.1 about the standard factorization $\operatorname{gari}\left(\mathrm{Sal}^{\bullet}, \mathrm{Sar}^{\bullet}\right)$ of bisymmetrals and bearing in mind that in the eupolar context the right factor $\operatorname{Sar}^{\bullet}$, due to homogeneity, is not only neg- but also pari-invariant, we arrive at the following picture:

$$
\begin{aligned}
& \ddot{\mathfrak{o}} \mathfrak{s s ^ { \bullet }}=\operatorname{gari}\left(\mathfrak{o s s}^{\bullet}, \mathfrak{s o} \ddot{\mathfrak{o}} \mathfrak{s}^{\bullet}\right)=\operatorname{gari}\left(\mathfrak{o s s} \mathfrak{s}^{\bullet}, \operatorname{expari}\left(\operatorname{lo} \ddot{\mathfrak{o}} \mathfrak{l}^{\bullet}\right)\right) \\
& \text { swap } \uparrow \quad \text { swap } \uparrow \text { swap } \uparrow \\
& \mathfrak{e s s}^{\bullet}=\operatorname{gari}\left(\ddot{\mathfrak{e}} \mathfrak{s s}^{\bullet}, \mathfrak{s} \ddot{\mathfrak{e}} \mathfrak{e s} \mathfrak{s}^{\bullet}\right)=\operatorname{gari}\left(\ddot{\mathfrak{e}} \mathfrak{s s} \mathfrak{s}^{\bullet}, \operatorname{expari}\left(\ddot{\mathfrak{k}} \mathfrak{e} l^{\bullet}\right)\right) \\
& \text { syap } \uparrow \quad \text { syap } \uparrow \quad \text { syap } \uparrow \\
& \mathfrak{o s s}^{\bullet}=\operatorname{gari}\left(\ddot{\mathfrak{o} s s^{\bullet}}, \mathfrak{s o ̈ o s} \mathfrak{s}^{\bullet}\right)=\operatorname{gari}\left(\ddot{\mathfrak{o s s}} \mathfrak{s}^{\bullet}, \operatorname{expari}\left(\ddot{l o} \mathfrak{o l} l^{\bullet}\right)\right) \\
& \text { swap } \uparrow \quad \text { swap } \uparrow \text { swap } \uparrow \\
& \ddot{\mathfrak{e}} \mathfrak{s s} \mathfrak{s}^{\bullet}=\operatorname{gari}\left(\mathfrak{e s s}^{\bullet}, \mathfrak{s e} \ddot{e} \mathfrak{s}^{\bullet}\right)=\operatorname{gari}\left(\mathfrak{e s s} \mathfrak{s}^{\bullet}, \operatorname{expari}\left(\operatorname{le} \ddot{e} l^{\bullet}\right)\right) .
\end{aligned}
$$

As second gari-factors we have here regular bisymmetrals $\mathfrak{s e \ddot { e } s} \mathfrak{s}^{\bullet}$, etc. that are themselves exponentials of regular bialternals $\mathfrak{l} \ddot{\mathfrak{e}} \ddot{\mathfrak{e}} \mathfrak{l}^{\bullet}$, etc. Both carry only even-length components, with a vanishing length- 2 component. ${ }^{56}$ Moreover, since the involution sap (product of swap and syap, in whichever order) turns $\mathfrak{s e \ddot { e } s} \mathfrak{s}^{\bullet}$ and $\mathfrak{s o} \ddot{\mathfrak{o} s}{ }^{\bullet}$ into their gari-inverses, we clearly have

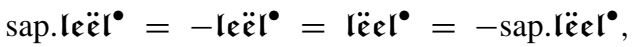

$$
\begin{aligned}
& \text { sap.lööl} \mathfrak{l}^{\bullet}=-\mathfrak{l o} \ddot{\mathfrak{o}} \mathfrak{l}^{\bullet}=\mathfrak{l o ̈ o} \mathfrak{l}^{\bullet}=- \text { sap.lö ol } \mathfrak{l}^{\bullet} \text {. }
\end{aligned}
$$

In the polar specialization, the picture becomes

$$
\begin{aligned}
& \operatorname{pal}^{\bullet}=\operatorname{gari}\left(\operatorname{par}^{\bullet}, \operatorname{ral}^{\bullet}\right)=\operatorname{gari}\left(\operatorname{par}^{\bullet}, \operatorname{expari}\left(\operatorname{liral}^{\bullet}\right)\right) \\
& \text { swap } \uparrow \quad \text { swap } \uparrow \text { swap } \uparrow \\
& \operatorname{pil}^{\bullet}=\operatorname{gari}\left(\operatorname{pir}^{\bullet}, \operatorname{ril}^{\bullet}\right)=\operatorname{gari}\left(\operatorname{pir}^{\bullet}, \operatorname{expari}\left(\operatorname{liril}^{\bullet}\right)\right) \\
& \text { syap } \uparrow \quad \text { syap } \uparrow \quad \text { syap } \uparrow \\
& \operatorname{par}^{\bullet}=\operatorname{gari}\left(\operatorname{pal}^{\bullet}, \operatorname{lar}^{\bullet}\right)=\operatorname{gari}\left(\operatorname{pal}^{\bullet}, \operatorname{expari}\left(\operatorname{lilar}^{\bullet}\right)\right) \\
& \text { swap } \uparrow \quad \text { swap } \uparrow \quad \text { swap } \uparrow \\
& \operatorname{pir}^{\bullet}=\operatorname{gari}\left(\operatorname{pil}^{\bullet}, \operatorname{lir}^{\bullet}\right)=\operatorname{gari}\left(\operatorname{pil}{ }^{\bullet}, \operatorname{expari}\left(\operatorname{lilir} \bullet^{\bullet}\right)\right)
\end{aligned}
$$

with

$$
\operatorname{gari}\left(\operatorname{lar}^{\bullet}, \operatorname{ral}^{\bullet}\right)=\operatorname{gari}\left(\operatorname{lir}^{\bullet}, \operatorname{ril}^{\bullet}\right)=1^{\bullet}
$$

and

$$
\operatorname{lilar}^{\bullet}=-\operatorname{liral}^{\bullet} \quad ; \quad \operatorname{lilir}^{\bullet}=- \text { liril }^{\bullet}
$$

To construct our first series of bialternals, we now have the choice between the components of infinitesimal generators such as $\operatorname{lilir}^{\bullet}$ or those of dilators such as dilir ${ }^{\bullet}$ or diril $l^{\bullet}$. Past experience suggests that the latter are to be preferred, and anyway the three systems $\left\{\right.$ lilir $\left._{2 r}^{\bullet}\right\},\left\{\right.$ dilir $\left._{2 r}^{\bullet}\right\},\left\{\right.$ diril $\left._{2 r}^{\bullet}\right\}$ generate exactly the same bialternal subalgebra of ARI.

\footnotetext{
${ }^{56}$ See Proposition 3.1.
} 
So, forgetting about $\operatorname{lilir}^{\bullet}$, let us look at the dilators $\operatorname{dilir}^{\bullet}$ and diril $^{\bullet}$ to decide which is simpler. Starting from the factorizations

$$
\operatorname{lir}^{\bullet}=\operatorname{gari}\left(\operatorname{ripil}^{\bullet}, \operatorname{pir}^{\bullet}\right) \quad ; \quad \operatorname{ril}^{\bullet}=\operatorname{gari}\left(\operatorname{ripir}^{\bullet}, \text { pil }^{\bullet}\right)
$$

or the more economical factorizations

$$
\operatorname{lir}^{\bullet}=\operatorname{gari}\left(\operatorname{ripil}_{\mathrm{ev}}^{\bullet}, \operatorname{pir}_{\mathrm{ev}}^{\bullet}\right) \quad ; \quad \operatorname{ril}^{\bullet}=\operatorname{gari}\left(\operatorname{ripir}_{\mathrm{ev}}^{\bullet}, \text { pil }_{\mathrm{ev}}^{\bullet}\right)
$$

and applying the rule (44) for dilator composition, we find respectively

$$
\begin{gathered}
\operatorname{dilir}^{\bullet}=\operatorname{adari}\left(\operatorname{ripir}^{\bullet}\right) .\left(\operatorname{diripil}^{\bullet}-\operatorname{diripir}^{\bullet}\right), \\
\operatorname{diril}^{\bullet}=\operatorname{adari}\left(\operatorname{ripil}^{\bullet}\right) .\left(\operatorname{diripir}^{\bullet}-\operatorname{diripil}^{\bullet}\right)
\end{gathered}
$$

and

$$
\begin{gathered}
\operatorname{dilir}^{\bullet}=\operatorname{adari}\left(\operatorname{ripir}_{\mathrm{ev}}^{\bullet}\right) .\left(\operatorname{diripil}_{\mathrm{ev}}^{\bullet}-\operatorname{diripir}_{\mathrm{ev}}^{\bullet}\right), \\
\operatorname{diril}^{\bullet}=\operatorname{adari}\left(\operatorname{ripil}_{\mathrm{ev}}^{\bullet}\right) .\left(\operatorname{diripir}_{\mathrm{ev}}^{\bullet}-\operatorname{diripil}_{\mathrm{ev}}^{\bullet}\right) .
\end{gathered}
$$

The identities (348) and (349) are unnecessarily wasteful, since they draw on all components, even and odd, of the central bisymmetrals to calculate the components $\operatorname{dilir}_{2 r}^{\bullet}$ and $\operatorname{diril}_{2 r}$, all even, of the bialternals. And of the two remaining identities, (351) is better than (350) since it involves, via the adari action, the bimould ripil $_{\mathrm{ev}}^{\bullet}$, which is much simpler than ripir $_{\mathrm{ev}}^{\bullet}{ }^{57}$

We have thus got hold of our first series of bialternals $\left\{\right.$ diril $\left.l_{2 r}^{\bullet} ; r \geq 2\right\}$ along with a probably optimal algorithm for their calculation. Indeed, using formula (42) and the key results (153) and (154) of Section 3, we can make the terms on the right-hand side of (351) wholly explicit. For the bimould part, we get an expansion in terms of elementary alternals

$$
\operatorname{diripir}_{\mathrm{ev}}^{\bullet}-\operatorname{diripil}_{\mathrm{ev}}^{\bullet}=\sum_{1 \geq r} \frac{2^{1-2 r}}{(2 r-1)(2 r+1)}\left(\mathrm{ki}_{2 r}^{\bullet}-\mathrm{ri}_{2 r}^{\bullet}\right)
$$

and for the operator part we have an equally simple expansion

$$
\operatorname{adari}\left(\operatorname{ripil}_{\mathrm{ev}}^{\bullet}\right)=\mathrm{id}+\sum \mathrm{Paj}^{2 r_{1}, \ldots, 2 r_{s}}\left[\prod_{j=1}^{j=s} \frac{2^{1-2 r_{j}}}{\left(2 r_{j}-1\right)\left(2 r_{j}+1\right)}\right] \underline{\operatorname{ari}}\left(r i_{2 \mathrm{r}_{1}}^{\bullet}\right) \ldots \underline{\operatorname{ari}}\left(r i_{2 r_{s}}^{\bullet}\right) .
$$

\section{Polar Bialternals: Second Main Source}

\subsection{Abstract Singulators}

To begin with, we must recall the construction of the "abstract" singulator senk that to any bisymmetral $\mathfrak{e s s}^{\bullet}$ associates (non-linearly) a linear operator

$$
\operatorname{senk}\left(\mathfrak{e s s}^{\bullet}\right)=\sum_{1 \leq r} \operatorname{senk}_{r}\left(\mathfrak{e s s}^{\bullet}\right)
$$

\footnotetext{
${ }^{57}$ In fact, diril ${ }^{\bullet}$ is not just simpler to calculate than dilir ${ }^{\bullet}$; it is also simpler in itself, in its coefficient structure, as can be seen from the extensive tables referred to in Section 18 and posted on our Webpage.
} 
whose "components" $\operatorname{senk}_{r}\left(\mathfrak{e s s}^{\bullet}\right)$ have the astonishing property of turning any length-1 bimould into a bialternal bimould of length $r$. That, however, comes at a price: every second time the bialternal so produced is identically 0 . More precisely,

$$
\begin{aligned}
\operatorname{senk}_{2 r}\left(\mathfrak{e s s}^{\bullet}\right) & : \mathrm{BIMU}_{1}^{\text {even }} \longrightarrow 0^{\bullet}, \\
\operatorname{senk}_{2 \mathrm{r}}\left(\mathfrak{e s s}^{\bullet}\right): & \mathrm{BIMU}_{1}^{\text {odd }} \longrightarrow \mathrm{BIMU}_{2 \mathrm{r}} \frac{\mathrm{al} / \mathrm{al}}{}, \\
\operatorname{senk}_{2 \mathrm{r}-1}\left(\mathfrak{e s s}^{\bullet}\right): & \mathrm{BIMU}_{1}^{\text {even }} \longrightarrow \mathrm{BIMU} \frac{\mathrm{al} / \mathrm{al}}{2 \mathrm{r}-1}, \\
\operatorname{senk}_{2 \mathrm{r}-1}\left(\mathfrak{e s s}^{\bullet}\right): & \mathrm{BIMU}_{1}^{\text {odd }} \longrightarrow 0^{\bullet} .
\end{aligned}
$$

Before constructing senk, let us recall the definition of $m u t$ (anti-action of $B I M U$ on itself) and adari (action of GARI on $A R I$ ):

$$
\begin{aligned}
\operatorname{mut}\left(\mathrm{B}^{\bullet}\right) \cdot \mathrm{A}^{\bullet} & :=\operatorname{mu}\left(\operatorname{invmu}\left(\mathrm{B}^{\bullet}\right), \mathrm{A}^{\bullet}, \mathrm{B}^{\bullet}\right), \\
\operatorname{adari}\left(\mathrm{B}^{\bullet}\right) \cdot \mathrm{A}^{\bullet} & :=\operatorname{logari}\left(\operatorname{gari}\left(\mathrm{B}^{\bullet}, \operatorname{expari}\left(\mathrm{A}^{\bullet}\right), \operatorname{invgari}\left(\mathrm{B}^{\bullet}\right)\right)\right) \\
& =\operatorname{gari}\left(\operatorname{preari}\left(B^{\bullet}, A^{\bullet}\right), \operatorname{invgari}\left(B^{\bullet}\right) .\right.
\end{aligned}
$$

We also require elementary operators that render any bimould neg- or push-invariant

$$
\begin{aligned}
\text { neginvar } & :=\mathrm{id}+\mathrm{neg}, \\
\text { pushinvar } & :=\sum_{0 \leq r}\left(\mathrm{id}+\mathrm{push}+\mathrm{push}^{2}+\ldots+\mathrm{push}^{r}\right) . \text { leng }_{r} .
\end{aligned}
$$

We can now enunciate the two equivalent definitions of senk

$$
\begin{aligned}
\operatorname{senk}\left(\mathfrak{e s s}^{\bullet}\right) \cdot \mathrm{S}^{\bullet} & :=\frac{1}{2} \text { neginvar. }\left(\operatorname{adari}\left(\mathfrak{e s s}^{\bullet}\right)\right)^{-1} \cdot \operatorname{mut}\left(\mathfrak{e s}^{\bullet}\right) . \mathrm{S}^{\bullet} \\
& =\frac{1}{2} \text { pushinvar.mut }\left(\text { neg } \cdot \mathfrak{e s s}^{\bullet}\right) \cdot \operatorname{garit}\left(\mathfrak{e} \mathfrak{s s}^{\bullet}\right) \cdot \mathrm{S}^{\bullet}
\end{aligned}
$$

The "components" $\operatorname{senk}_{\mathrm{r}}\left(\mathfrak{e s s}^{\bullet}\right)$ are of course defined in the only possible way

$$
\operatorname{senk}_{r}\left(\mathfrak{e s s}^{\bullet}\right) . S^{\bullet}:=\operatorname{leng}_{\mathrm{r}} \cdot \operatorname{senk}\left(\mathfrak{e s s}^{\bullet}\right) . S^{\bullet}
$$

with leng $_{r}$ denoting the natural projection of BIMU onto $B I M U_{r}$.

The magic properties of senk result from its remarkable behavior under the swap transform 58

$$
\begin{aligned}
\operatorname{swap} . \operatorname{senk}\left(\mathfrak{e s s}^{\bullet}\right) . S^{\bullet}:=\operatorname{senk}\left(\text { pari. } \ddot{\mathfrak{o s s}} \mathfrak{s}^{\bullet}\right) . \operatorname{swap} . \mathrm{S}^{\bullet} \\
\text { swap.senk }_{\mathrm{r}}\left(\mathfrak{e s s}^{\bullet}\right) . \mathrm{S}^{\bullet}:=(-1)^{\mathrm{r}-1} \operatorname{senk}_{\mathrm{r}}\left(\ddot{\mathfrak{o}} \mathfrak{s s}^{\bullet}\right) . \operatorname{swap} . \mathrm{S}^{\bullet}
\end{aligned}
$$

\footnotetext{
${ }^{58}$ The $(-1)^{r-1}$ in (366) is no misprint: the operator $\operatorname{senk}_{r}\left(\mathbf{e s s}^{\bullet}\right)$ involves various products of components $\mathfrak{e s s}_{r_{i}}$ and for each such product the total length $\sum r_{i}$ is $r-1$, not $r$.
} 


\subsection{The Polar Singulators slank and srank}

Substituting pil $^{\bullet}$ or pir ${ }^{\bullet}$ for $\mathfrak{e s s}^{\bullet}$ in senk, we get two operators slink and srink ${ }^{59}$

$$
\begin{aligned}
& \text { slink. } S^{\bullet}:=\frac{1}{2} \text { neginvar. }\left(\operatorname{adari}\left(\operatorname{pil}^{\bullet}\right)\right)^{-1} \cdot \operatorname{mut}\left(\operatorname{pil} 1^{\bullet}\right) . S^{\bullet} \\
& =\frac{1}{2} \text { pushinvar } \cdot \operatorname{mut}\left(\text { neg.pil }{ }^{\bullet}\right) \cdot \operatorname{garit}\left(\text { pil }^{\bullet}\right) \cdot \mathrm{S}^{\bullet}, \\
& \text { srink. } S^{\bullet}:=\frac{1}{2} \text { neginvar . }\left(\operatorname{adari}\left(\operatorname{pir}^{\bullet}\right)\right)^{-1} \cdot \operatorname{mut}\left(\operatorname{pir}^{\bullet}\right) . S^{\bullet} \\
& =\frac{1}{2} \text { pushinvar } \cdot \operatorname{mut}\left(\text { neg.pir } \mathbf{p}^{\bullet}\right) \cdot \operatorname{garit}\left(\operatorname{pir}^{\bullet}\right) \cdot \mathrm{S}^{\bullet},
\end{aligned}
$$

whose "components" slink $k_{r}$ and srink $k_{r}$ turn arbitrary, entire-valued length-1 bimoulds into bialternal, singular-valued length- $r$ bimoulds. This property makes slink $k_{r}$ and $\operatorname{srink}_{r}$ extremely useful in multizeta algebra, in the back-and-forth known as singularizationdesingularization.

\subsection{The Second Series of Bialternals}

Our aim here, however, is different: we want to produce eupolar bialternals, i.e., bialternal elements of Flex $(P i)$. Here, the "singuland" (i.e., that on which the singulator acts) can only be $P i^{\bullet}$, and so, in view of (353)-(356), the "singulate" (i.e., the bialternal fruit of the operation) can and in fact will be nonzero only in the situation (354). So we have no choice but to set

$$
\begin{aligned}
& \operatorname{visli}_{2 r}^{\bullet}:=\operatorname{slink}_{2 \mathrm{r}} \cdot \mathrm{Pi}^{\bullet}, \\
& \operatorname{visri}_{2 r}^{\bullet}:=\operatorname{srink}_{2 \mathrm{r}} \cdot \mathrm{Pi}^{\bullet} .
\end{aligned}
$$

\subsection{Relations Between the Two Series of Bialternals}

Like with the two equivalent systems $\left\{\operatorname{diri}_{2 r}^{\bullet}\right\}$ and $\left\{\right.$ dilir $\left._{2 r}^{\bullet}\right\}$ of the preceding section, it is easy to show that the new systems $\left\{v i s l i_{2 r}^{\bullet}\right\}$ and $\left\{v i s r i_{2 r}\right\}$ are also equivalent, in the sense of generating one and the same bialternal subalgebra of $A R I$. So we shall retain only $\left\{v i s l i_{2 r}^{\bullet}\right.$, since it can be shown to be simpler than $\left\{\right.$ visri $\left._{2 r}^{\bullet}\right\}$, much as $\left\{\right.$ diri $\left._{2 r}^{\bullet}\right\}$ was simpler than $\left\{\operatorname{dilir}_{2 r}^{\bullet}\right\}$.

The only questions left are these:

(i) How do the systems $\left\{\operatorname{diri}_{2 r}^{\bullet}\right\}$ and $\left\{\right.$ visli $i_{2 r}^{\bullet}$ compare?

(ii) Do they, together, generate all eupolar bialternals?

The answer to the second question is probably no, but this is no more than a hunch. The answer to the first question is not clear either: up to length 10, the two systems are equivalent; at length 12, they produce a distinct generator each; but at length 14, they do not. And what happens thereafter is anybody's guess.

\footnotetext{
${ }^{59}$ In view of (365), subsituting $\mathrm{pal}^{\bullet}$ or $\mathrm{par}^{\bullet}$ for $\mathfrak{e s s}^{\bullet}$ in $\operatorname{senk}$ would produce nothing new. It would just yield (up to sign) the swap transforms of slink and srink.
} 


\section{Polar Algebra and Subalgebras}

Warning: from here on, the exposition becomes less systematic and the paper takes a more exploratory turn. It mixes proof-backed statements, conjectures, and mere "observed facts," while making clear in each case which is which.

The six main subspaces of Flex $(\mathfrak{E})$ are ${ }^{60}$

$\operatorname{Flex}^{\text {sap }}(\mathfrak{E})$, consisting of all sap - invariant bimoulds.

Flex ${ }^{\overline{p u s}}(\mathfrak{E})$, consisting of all pus - variant bimoulds.

Flex ${ }^{\text {push }}(\mathfrak{E})$, consisting of all push-invariant bimoulds.

$\operatorname{Flex}^{\mathrm{al}}(\mathfrak{E})$, consisting of all alternal bimoulds.

$\operatorname{Flex}^{\mathrm{al} / \mathrm{push}}(\mathfrak{E})$, consisting of all alternal and $\mathrm{push}$ - invariant bimoulds.

Flex $\underline{\underline{a}} / \underline{\mathrm{a}}(\mathfrak{E})$, consisting of all bialternal bimoulds.

All these subspaces except the first (sap-invariants) are stable under ari and define as many subalgebras. On the other hand, only the fourth (alternals) is stable under $l u$. This again shows how much more flexible, versatile and interesting the flexion operations are. Remarkably, neither the pus-invariant subspace Flex ${ }_{r}^{\text {pus }}$ nor the push-variant subspace Flex ${ }_{r}^{\overline{p u s h}}$ are stable under ari, let alone $l u .{ }^{61}$

Here is a table with the dimensions, up to $r=14$, of the length- $r$ components of these subspaces or subalgebras.

\begin{tabular}{l|lllllll}
$r$ & Flex & Flex $_{r}^{\text {sap }}$ & Flex $_{r}^{\overline{p u s}}$ & Flex $_{r}^{\text {push }}$ & Flex $_{r}^{\text {al }}$ & Flex $_{r}^{\text {al } / \text { push }}$ & Flex ${ }_{r}^{\mathrm{al} / \underline{a l}}$ \\
1 & 1 & 1 & 0 & 0 & 1 & 0 & 0 \\
2 & 2 & 1 & 1 & 0 & 1 & 0 & 0 \\
3 & 5 & 3 & 3 & 0 & 2 & 0 & 0 \\
4 & 14 & 7 & 9 & 2 & 4 & 1 & 1 \\
5 & 42 & 22 & 28 & 4 & 9 & 1 & 0 \\
6 & 132 & 66 & 90 & 18 & 20 & 4 & 1 \\
7 & 429 & 217 & 297 & 48 & 48 & 7 & 0 \\
8 & 1430 & 715 & 1001 & 156 & 115 & 17 & 1 \\
9 & 4862 & 2438 & 3432 & 472 & 286 & 36 & 0 \\
10 & 16796 & 8398 & 11934 & 1526 & 719 & 88 & 2 \\
11 & 58786 & 29414 & 41990 & 4852 & 1842 & 196 & 0 \\
12 & 208012 & 104006 & 149226 & 16000 & 4766 & 481 & $\geq 3$ \\
13 & 742900 & 371516 & 534888 & 52940 & 12486 & 1148 & 0 \\
14 & 2674440 & 1337220 & 1931540 & 178276 & 32973 & 2838 & $\geq 3$
\end{tabular}

All these dimensions have remarkable combinatorial interpretations, mostly in terms of special trees with $r$ or $r-1$ nodes. See [7].

- $\operatorname{dim}\left(\right.$ Flex $\left._{r}(\mathfrak{E})\right)=\frac{(2 r) !}{r !(r+1) !}$. For two distinct interpretations and the corresponding bases, see Remark 1 below.

- $\operatorname{dim}\left(\right.$ Flex $\left._{r}^{\text {sap }}(\mathfrak{E})\right)=\frac{1}{2} \operatorname{dim}\left(\right.$ Flex $\left._{r}^{\text {sap }}\right)$, resp. $=\frac{1}{2} \operatorname{dim}\left(\right.$ Flex $\left._{r}\right)+\operatorname{dim}\left(\right.$ Flex $\left._{(r-1) / 2}\right)$ if $r$ is even, resp. odd.

\footnotetext{
${ }^{60}$ Recall that sap $:=$ swap.syap $=$ syap.swap and that a bimould $A^{\bullet}$ in $B I M U_{r}$ is said to be pus-variant if and only if $\left(i d+p u s+p^{2} s^{2}+\ldots p u s^{r-1}\right) \cdot A^{\bullet}=0$.

${ }^{61}$ This underscores the "complementarity" between pus (a circular permutation of order $r$ in the short notation) and push (a circular permutation of order $r$ in the long notation).
} 
- $\operatorname{dim}\left(\right.$ Flex $\left._{r}^{\overline{\mathrm{pus}}}(\mathfrak{E})\right)=\frac{3(2 r-2) !}{(r+1) !(r-2) !}$. The sequence occurs in the Online Encyclopedia of Integer Sequences under A000245 with a number of combinatorial interpretations.

- $\operatorname{dim}\left(\operatorname{Flex}_{r}^{\text {push }}(\mathfrak{E})\right)=2 \frac{(2 r) !}{r !(r+1) !}-\frac{1}{2 r+2} \sum_{d \mid r+1} \phi(d) \frac{((2 r+2) / d) !}{((r+1) / d) !((r+1) / d) !}$. This formula is due to F. Chapoton, who used it to solve a different problem, but with a combinatorial interpretation easily translatable into ours. See [1] or item A106520 in the Online Encyclopedia of Integer Sequences.

- $\operatorname{dim}\left(\right.$ Flex $\left._{r}(\mathfrak{E})\right)=$ number $\beta(r)$ of non-ordered ${ }^{62}$ rooted trees with $r$ nodes. ${ }^{63}$ For numerous alternative interpretations and formulae for inductive calculation, see A000081 in the Online Encyclopedia of Integer Sequences. Thus, the generating series $B(x):=\sum_{0<r} \beta(r) x^{r}$ verifies $B(x)=x \exp \left(\sum_{1 \leq k} \frac{1}{k} B\left(x^{k}\right)\right)$. For a combinatorial interpretation directly related to our problem, see Remark 2 below.

- $\operatorname{dim}\left(\operatorname{Flex}_{r}^{\text {al/push }}(\mathfrak{E})\right)$. Though there is no known closed formula, this again appears to coincide with a sequence investigated by F. Chapoton (see A098091 in the Online Encyclopedia of Integer Sequences) but with a combinatorial interpretation ${ }^{64}$ that doesn't make the connection obvious.

- $\operatorname{dim}\left(\right.$ Flex $\left._{r}^{\mathrm{al} / \mathrm{al}}(\mathfrak{E})\right)=$ unknown at the moment for $r \geq 16$. See Section 10.4.

Remark 1 Bases of Flex $(\mathfrak{E})$.

As is well known, the Catalan numbers $\operatorname{dim}\left(\right.$ Flex $\left._{r}(\mathfrak{E})\right)=\frac{(2 r) !}{r !(r+1) !}$ are capable of two main tree-theoretic interpretations:

(i) As counting the binary trees with $r$-nodes,

(ii) As counting the ordered trees ${ }^{65}$ with $r$-nodes. ${ }^{66}$

There exists a basis $\left\{\mathfrak{e}_{t}^{\bullet}\right\}$ naturally indexed by the binary trees $t$ : see Section 1.6.

There also exist two bases $\left\{\mathfrak{e m}_{t}^{\bullet}\right\}$ and $\left\{\mathfrak{e n}_{t}^{\bullet}\right\}$ indexed by the ordered trees of the second interpretation. Indeed, let $t$ be a $s$-rooted tree consisting of an ordered system of $s$ one-rooted trees $\boldsymbol{t}_{j}$; and let $\boldsymbol{t}_{*}$ be the one-rooted tree that results from attaching each $\boldsymbol{t}_{j}$ to a common root. ${ }^{67}$ The inductive definition then reads

$$
\begin{aligned}
& \mathfrak{e m}_{t}^{\bullet}:=\operatorname{mu}\left(\mathfrak{e m}_{t_{1}}^{\bullet}, \ldots, \mathfrak{e m} \mathfrak{m}_{t_{s}}^{\bullet}\right) ; \mathfrak{e m}_{t_{*}}^{\bullet}:=\operatorname{amit}\left(\mathfrak{e} \mathfrak{m}_{t}^{\bullet}\right) . \mathfrak{E}^{\bullet}, \\
& \mathfrak{e n} \mathfrak{n}_{t}^{\bullet}:=\operatorname{mu}\left(\mathfrak{e n} \mathfrak{n}_{t_{1}}^{\bullet}, \ldots, \mathfrak{e n} \mathfrak{n}_{t_{s}}^{\bullet}\right) ; \mathfrak{e n}_{\boldsymbol{t}_{*}}^{\bullet}:=\operatorname{anit}\left(\mathfrak{e} \mathfrak{n}_{\boldsymbol{t}}^{\bullet}\right) . \mathfrak{E}^{\bullet}
\end{aligned}
$$

starting of course from $\mathfrak{e m}_{t_{0}}^{\bullet}=\mathfrak{e m}_{t_{0}}^{\bullet}:=\mathfrak{E}^{\bullet}$ for the one-node, one-root tree $\boldsymbol{t}_{0}$. The two systems $\left\{\mathfrak{e m}_{\boldsymbol{t}}^{\bullet} ; \operatorname{nodes}(\boldsymbol{t})=r\right\}$ and $\left\{\mathfrak{e n}_{\boldsymbol{t}}^{\bullet} ; \operatorname{nodes}(\boldsymbol{t})=r\right\}$ are each a basis ${ }^{68}$ of $\operatorname{Flex}_{r}(\mathfrak{E})$.

\footnotetext{
${ }^{62}$ The relative position of the various branches issueing from a given node is indifferent.

${ }^{63}$ Counting the root as a node.

${ }^{64}$ According to $\mathrm{F}$. Chapotion, these are the graded dimensions of the spaces of invariant bilinear forms on the free pre-Lie algebra on one generator.

${ }^{65}$ Several branches may issue from one and the same node, and their planar disposition, from left to right, matters.

${ }^{66}$ Several roots are allowed in these "trees". Some speak of bushes or forests instead.

${ }^{67}$ Distinct from the original roots of each $\boldsymbol{t}_{j}$.

${ }^{68}$ Note that the systems $\left\{\mathfrak{e m}_{t}^{\bullet}\right\}$ and $\left\{\mathfrak{e n}_{t}^{\bullet}\right\}$ are quite distinct from the similar-looking systems in Section 2.1. The latter span much smaller subspaces.
} 
However, the system $\left\{\mathfrak{e r}_{\boldsymbol{t}}^{\bullet}\right.$; nodes $\left.(\boldsymbol{t})=r\right\}$ similarly constructed but with arit in place of amit or anit defines no basis. ${ }^{69}$ Worse still, Flex $(\mathfrak{E})$ cannot be generated from $\mathfrak{E}^{\bullet}$ under repeated use of the sole operations $l u$ and arit (much less under $l u$ and ari).

Remark 2 Basis of Flex ${ }_{r}^{a l}(\mathfrak{E})$.

Let $\boldsymbol{\theta}:=\left\{\overline{\boldsymbol{\theta}_{1}}, \ldots, \boldsymbol{\theta}_{s}\right\}$ be the unordered rooted tree obtained by attaching $s$ unordered rooted trees $\boldsymbol{\theta}_{j}$ to a common root. Then the inductive rule ${ }^{70}$

$$
\mathfrak{e r r}_{\boldsymbol{\theta}}^{\bullet}:=\sum_{\sigma \in \mathcal{S}_{s}} \overrightarrow{\mathrm{lu}}\left(\operatorname{arit}\left(\mathfrak{e r r}_{\boldsymbol{\theta}_{\sigma(1)}^{\bullet}}^{\bullet}\right) \cdot \mathfrak{E}^{\bullet}, \mathfrak{e r r}_{\boldsymbol{\theta}_{\sigma(2)}}^{\bullet}, \ldots, \mathfrak{e r r}_{\boldsymbol{\theta}_{\sigma(s)}}^{\bullet}\right)
$$

produces, for each $r$, a system $\left\{\mathfrak{e r r}_{\boldsymbol{\theta}}^{\bullet} ; \operatorname{nodes}(\boldsymbol{\theta})=r\right\}$ consisting of bimoulds that are alternal of length $r$ (obvious); have the right indexation and so too the right cardinality (obvious); are linearly independent (non obvious); and therefore constitute a basis of Flex ${ }_{r}^{a l}(\mathfrak{E})$. This is a rather unusual situation, given that most free Lie algebras ${ }^{71}$ possess no privileged natural basis.

\section{Interplay of the $l u$ and ari Structures}

(i) As $l u$-algebras, both Flex ${ }^{a l}(\mathfrak{E})$ and Flex(E) are freely generated by a well-defined number of prime generators $\mathfrak{g e}_{r, i}^{\bullet}$ taken in each component space Flex al $(\mathfrak{E})$ or Flex $(\mathfrak{E})$.

(ii) As ari-algebras, both Flex ${ }^{a l}(\mathfrak{E})$ and Flex(E) decompose as

$$
\begin{aligned}
\operatorname{Flex}^{a l}(\mathfrak{E}) & =\operatorname{Flex}^{\mathrm{al}}(\mathfrak{r} \mathfrak{e}) \oplus \operatorname{Flex}_{\text {free }}^{a l}(\mathfrak{E}), \\
\operatorname{Flex}(\mathfrak{E}) & =\operatorname{Flex}^{a l}(\mathfrak{r} \mathfrak{e}) \oplus \operatorname{Flex}_{\text {free }}(\mathfrak{E}) .
\end{aligned}
$$

The elementary subalgebra Flexal $(\mathfrak{r} \mathfrak{e})$ is generated (and spanned) by the selfreproducing alternals $\mathfrak{r} \mathfrak{e}_{r}^{\bullet}$. All its components Flex $x_{r}^{a l}(\mathfrak{r} \mathfrak{e})$ are one-dimensional. The algebra Flex free $(\mathfrak{E})$, resp. Flex free $(\mathfrak{E})$, is freely generated by a well-defined number of primary generators $\mathfrak{f} \mathfrak{e}_{r, i}^{\bullet}$ taken in each Flex ${ }_{r}^{a l}(\mathfrak{E})$, resp. Flex $x_{r}(\mathfrak{E})$, and supplemented by secondary generators of the form

$$
\overrightarrow{a r i}\left(\mathfrak{f} \mathfrak{e}_{r_{0}}^{\bullet}, \mathfrak{r} \mathfrak{e}_{r_{1}}^{\bullet}, \ldots, \mathfrak{r} \mathfrak{e}_{r_{s}}^{\bullet}\right) \text { with } \quad r_{0}+r_{1}+\ldots r_{s}=r
$$

with only non-increasing (or non-decreasing, if one so prefers ${ }^{72}$ ) integer sequences $\left(r_{1}, \ldots, r_{s}\right)$.

The following table carries for each length- $r$ component of $\operatorname{Flex}_{\text {free }}^{a l}(\mathfrak{E}), \operatorname{resp}_{\text {. Flex }}$ free $(\mathfrak{E})$ :

(i) The total dimension $\delta_{r}$, resp. $d_{r}$,

(ii) The number $\delta_{r}^{*}$, resp. $d_{r}^{*}$, of primary generators,

(iii) The number $\delta_{r}^{* *}$, resp. $d_{r}^{* *}$, of all generators (primary and secondary).

\footnotetext{
${ }^{69}$ There appear linear dependence relations between the $\mathfrak{e r}_{t}^{\bullet}$ as soon as $r=5$.

${ }^{70}$ As usual, we get the induction started by setting $\mathfrak{e r r}_{\boldsymbol{\theta}_{0}}^{\bullet}:=\mathfrak{E}^{\bullet}$ for the one-node one-root tree $\boldsymbol{\theta}_{0}$.

${ }^{71}$ As a $l u$-algebra, Flex ${ }^{a l}(\mathfrak{E})$ is free, and very nearly free as an ari-algebra. See Section 12.

${ }^{72}$ Working out the conversion rules between the two systems (376) that correspond to non-increasing or non-decreasing sequences, and finding a compact expression for these rules, is a wholesome exercise on moulds.
} 


$\begin{array}{rrrrrrr}\mid & \text { Flex }_{\mathrm{r}}^{\mathrm{al}} \mid & \text { Flex }_{\mathrm{r}}^{\mathrm{al}} \mid & \text { Flex }_{\mathrm{r}}^{\mathrm{al}} \mid & \text { Flex }_{\mathrm{r}} \mid & \text { Flex }_{\mathrm{r}} \mid & \text { Flex }_{\mathrm{r}} \\ r \mid & \delta_{r} \mid & \delta_{r}^{*} \mid & \delta_{r}^{* *} \mid & d_{r} \mid & d_{r}^{*} \mid & d_{r}^{* *} \\ \mid & \ldots \mid & \ldots \mid & \ldots \mid & \ldots \mid & \ldots \mid & \ldots \\ 1 \mid & 1 \mid & 0 \mid & 0 \mid & 1 \mid & 0 \mid & 0 \\ 2 \mid & 1 \mid & 0 \mid & 0 \mid & 2 \mid & 1 \mid & 1 \\ 3 \mid & 2 \mid & 1 \mid & 1 \mid & 5 \mid & 3 \mid & 4 \\ 4 \mid & 4 \mid & 2 \mid & 3 \mid & 14 \mid & 8 \mid & 13 \\ 5 \mid & 9 \mid & 4 \mid & 8 \mid & 42 \mid & 20 \mid & 37 \\ 6 \mid & 20 \mid & 8 \mid & 19 \mid & 132 \mid & 62 \mid & 112 \\ 7 \mid & 48 \mid & 17 \mid & 44 \mid & 429 \mid & 187 \mid & 335 \\ 8 \mid & 115 \mid & 41 \mid & 103 \mid & 1430 \mid & 619 \mid & 1062 \\ 9 \mid & 286 \mid & 98 \mid & 242 \mid & 4862 \mid & 2049 \mid & 3432 \\ 10 \mid & 719 \mid & 250 \mid & 586 \mid & 16796 \mid & 6998 \mid & 11451 \\ 11 \mid & 1842 \mid & 631 \mid & 1437 \mid & 58786 \mid & 24186 \mid & 38944 \\ 12 \mid & 4766 \mid & 1645 \mid & 3616 \mid & 208012 \mid & 84673 \mid & 134696 \\ 13 \mid & 12486 \mid & 4285 \mid & 9216 \mid & 742900 \mid & 299445 \mid & 471911 \\ 14 \mid & 32973 \mid & 11338 \mid & 23884 \mid & 2674440 \mid & 1065675 \mid & 1668516\end{array}$

\section{Alternal Codegrees and Alternality Grids}

\subsection{Loose and Strict Alternality Codegrees}

A bimould $A^{\bullet} \in B I M U_{r}$ is said to have loose alternality codegree $d$ if the identity ${ }^{73}$

$$
\sum_{\boldsymbol{w} \in \operatorname{sha}\left(\boldsymbol{w}^{1}, \ldots, \boldsymbol{w}^{d+1}\right)} A^{w}=0 \quad\left(\forall \boldsymbol{w}, \forall \boldsymbol{w}^{i} \neq \emptyset\right)
$$

holds for all systems $\left\{\boldsymbol{w}^{1}, \ldots, \boldsymbol{w}^{d+1}\right\}$, and it is said to have strict alternality codegree $d$ if the identity does not always hold for $d-1$. Alternality in the usual sense corresponds to $d=1$. We speak here of codegrees rather than degrees, because the notion is clearly dual to that of "differential" degree. ${ }^{74}$

The (strict) codegree behaves additively under "products" such as $m u$ or preari, but with a unit drop in the case of 'brackets' like $l u$ or ari:

$$
\begin{aligned}
& C^{\bullet}=\operatorname{mu}\left(A^{\bullet}, B^{\bullet}\right) \quad \Longrightarrow \operatorname{codeg}^{a l}\left(C^{\bullet}\right)=\operatorname{codeg}^{a l}\left(A^{\bullet}\right)+\operatorname{codeg}^{a l}\left(B^{\bullet}\right), \\
& C^{\bullet}=\operatorname{preari}\left(A^{\bullet}, B^{\bullet}\right) \Longrightarrow \operatorname{codeg}^{a l}\left(C^{\bullet}\right)=\operatorname{codeg}^{a l}\left(A^{\bullet}\right)+\operatorname{codeg}^{a l}\left(B^{\bullet}\right), \\
& C^{\bullet}=\operatorname{lu}\left(A^{\bullet}, B^{\bullet}\right) \quad \Longrightarrow \operatorname{codeg}^{a l}\left(C^{\bullet}\right) \leq \operatorname{codeg}^{a l}\left(A^{\bullet}\right)+\operatorname{codeg}^{a l}\left(B^{\bullet}\right)-1, \\
& C^{\bullet}=\operatorname{ari}\left(A^{\bullet}, B^{\bullet}\right) \quad \Longrightarrow \operatorname{codeg}^{a l}\left(C^{\bullet}\right) \leq \operatorname{codeg}^{a l}\left(A^{\bullet}\right)+\operatorname{codeg}^{a l}\left(B^{\bullet}\right)-1 .
\end{aligned}
$$

\subsection{Filtration of Flex $_{r}(\mathfrak{E})$}

Consider the filtration

$$
\text { Flex }_{r}(\mathfrak{E})=\text { Flex }_{r}^{(r)}(\mathfrak{E}) \supset \text { Flex }_{r}^{(r-1)}(\mathfrak{E}) \supset \ldots \text { Flex }_{r}^{(2)}(\mathfrak{E}) \supset \text { Flex }_{r}^{(1)}(\mathfrak{E})
$$

\footnotetext{
${ }^{73}$ Recall that $\operatorname{sh} a\left(\boldsymbol{w}^{1}, \ldots, \boldsymbol{w}^{d+1}\right)$ denotes the set of all $\boldsymbol{w}$ that result from shuffling the various $\boldsymbol{w}^{i}$.

${ }^{74}$ Think of mould-comould contractions $\sum A^{w_{1}, \ldots, w_{r}} \Delta_{w_{r}} \ldots \Delta_{w_{1}}$, with inputs $\Delta_{w_{i}}$ freely generating a Lie algebra. Besides, as $d$ increases, $A^{\bullet}$ becomes "less alternal." not more. So it would be jarring to speak of alternality degree here.
} 
of Flex $x_{r}(\mathfrak{E})$ into subspaces Flex ${ }_{r}^{(d)}(\mathfrak{E})$ consisting of all elements of (loose) alternal codegree $d$. The following (incomplete) table mentions, for each $r$, the dimensions $a l_{r}^{d}$ of the corresponding gradation:

$$
\begin{aligned}
& \mathrm{al}_{r}^{d}:=\mathrm{Al}_{r}^{d}-\mathrm{Al}_{r}^{d-1} \quad \text { with } \quad \mathrm{Al}_{r}^{d}:=\operatorname{dim}\left(\text { Flex }_{r}^{(d)}(\mathfrak{E})\right) \\
& r \mid \begin{array}{r|rrrrrrrr}
d \mid & 1 & 2 & 3 & 4 & 5 & 6 & 7 & 8 \\
\mid \text { total } \mid & \ldots & \ldots & \ldots & \ldots & \ldots & \ldots & \ldots & \ldots
\end{array} \\
& 1|1| 1 \\
& 2|2| \quad 1 \quad 1
\end{aligned}
$$

\begin{tabular}{|c|c|c|c|c|c|c|c|c|c|c|c|c|c|c|c|}
\hline 8 & 7 & 6 & 5 & 4 & 3 & 2 & $1 \mid$ & $r \mid$ & 1 & 2 & 3 & 4 & 5 & & 78 \\
\hline & & & $\cdots$ & $\cdots$ & $\ldots$ & $\cdots$ & \begin{tabular}{c|}
$\ldots$ \\
1 \\
1 \\
1 \\
0
\end{tabular} & $\begin{array}{l}1^{ \pm} \\
1^{+} \\
1^{-}\end{array}$ & $\begin{array}{c}\ldots \\
0 \\
0 \\
0\end{array}$ & & $\cdots$ & & & & \\
\hline & & & & & & 2 & $0 \mid$ & $1^{ \pm}$ & 0 & 0 & & & & & \\
\hline & & & & & & 1 & $0 \mid$ & $2^{+1}$ & 0 & 0 & & & & & \\
\hline & & & & & & 1 & $0 \mid$ & $2^{-1}$ & 0 & 0 & & & & & \\
\hline & & & & & 2 & 3 & 0 & $3^{ \pm}$ & 0 & 0 & 0 & & & & \\
\hline & & & & & 1 & 2 & 0 & $3^{+1}$ & 0 & 0 & 0 & & & & \\
\hline & & & & & 1 & 1 & $0 \mid$ & $3^{-1}$ & 0 & 0 & 0 & & & & \\
\hline & & & & 2 & 6 & 5 & 1 & $4^{ \pm}$ & 1 & 1 & 0 & 0 & & & \\
\hline & & & & 1 & 3 & 3 & 0 & $4^{+}$ & 0 & 1 & 0 & 0 & & & \\
\hline & & & & 1 & 3 & 2 & $1 \mid$ & $4^{-1}$ & 1 & 0 & 0 & 0 & & & \\
\hline & & & 2 & 8 & 23 & 9 & $0 \mid$ & $5^{ \pm}$ & 0 & 2 & 2 & 0 & 0 & & \\
\hline & & & 1 & 4 & 12 & 5 & 0 & $5^{+}$ & 0 & 1 & 1 & 0 & 0 & & \\
\hline & & & 1 & 4 & 11 & 4 & 01 & $5^{-1}$ & 0 & 1 & 1 & 0 & 0 & & \\
\hline & & & 10 & 40 & 68 & 17 & $1 \mid$ & $6^{ \pm}$ & 1 & 5 & 8 & 4 & 0 & 0 & \\
\hline & & 1 & 5 & 20 & 32 & 8 & 01 & $6^{+}$ & 0 & 2 & 5 & 2 & 0 & 0 & \\
\hline & & 1 & 5 & 20 & 30 & 9 & $1 \mid$ & $6^{-1}$ & 1 & 3 & 3 & 2 & 0 & 0 & \\
\hline & 21 & 26 & 60 & 154 & 186 & 15 & $0 \mid$ & $7^{ \pm}$ & 0 & & 241 & 16 & 4 & 0 & 0 \\
\hline & 1 & 63 & 30 & 77 & 96 & 7 & 0 & $7^{+} \mid$ & 0 & & & & & 0 & 0 \\
\hline & 1 & 63 & 30 & 77 & 90 & 8 & $0 \mid$ & $7^{-1}$ & 0 & & & & & o & 0 \\
\hline 21 & 48 & 34 & & & & & 1 & $8^{ \pm}$ & 1 & & & & & & $\begin{array}{ll}0 & 0\end{array}$ \\
\hline 1 & 44 & 2 & & & & & 0 & $8^{+1}$ & 0 & & & & & & 0 \\
\hline 114 & 44 & 2 & & & & & 1 & $8^{-1}$ & 1 & & & & & & $\begin{array}{ll}0 & 0\end{array}$ \\
\hline
\end{tabular}

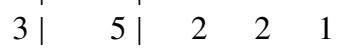

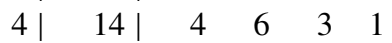

$$
\begin{aligned}
& \begin{array}{l|l|lllll}
5 \mid & 42 \mid & 9 & 16 & 12 & 4 & 1
\end{array} \\
& \begin{array}{l|l|llllll}
6 & 132 & 20 & 47 & 39 & 20 & 5 & 1
\end{array} \\
& \begin{array}{l|l|lllllll|}
7 & 429 \mid & 48 & 127 & 141 & 76 & 30 & 6 & 1
\end{array} \\
& 8 \text { | } 1430 \mid \begin{array}{llllllll}
115 & ? & ? & 130 & 42 & 7 & 1
\end{array} \\
& \mathrm{al}_{r}^{r-0}=1 \\
& \mathrm{al}_{r}^{r-1}=r-1 \\
& \mathrm{al}_{r}^{r-2}=(r-2)(r-1) \\
& \mathrm{al}_{r}^{r-3}=\frac{1}{2}(r-3)\left(r^{2}-r-4\right) \\
& \mathrm{al}_{r}^{r-4}=(r-4) \ldots
\end{aligned}
$$


Remark There are two main motivations for investigating the subspaces Flex ${ }_{r}^{(d)}$ with their dimensions $A l_{r}^{d}$ and the quotients flex $_{r}^{(d)}:=$ Flex $_{r}^{(d)} /$ Flex $_{r}^{(d-1)}$ with their dimensions $a l_{r}^{d}$. Firstly, despite the obvious primacy which the alternals (i.e., the bimoulds of flex $_{r}^{(1)}:=$ Flex $_{r}^{(1)}$ ) enjoy over their weaker analogues (i.e., the bimoulds of higher alternality codegrees), the latter are also deserving of attention, as they naturally occur in various constructions. But the second, even more compelling reason is this: it is often relatively easy to produce free generating systems $\mathcal{S}_{r}^{d}$ for subspaces subflex ${ }_{r}^{(d)} \subset$ flex $_{r}^{(d)}$, and then to check that the partial dimensions add up to the total dimension

$$
\operatorname{dim}\left(\text { Flex }_{r}\right)=\sum_{d} \operatorname{dim}\left(\text { subflex }_{r}^{(d)}\right) .
$$

This automatically proves the identities subflex ${ }_{r}^{(d)}=$ flex $_{r}^{(d)}$ for all $d$, and yields the corresponding dimensions. On the other hand, establishing these identities directly and separately (even only one of them, say for $d=1$, if we decided to restrict our attention to the sole alternals) would, paradoxically, be harder than establishing them simultaneously for all $d$ !

\section{Bialternal Codegrees and Bialternality Grids}

\subsection{Bialternal Codegree}

The bialternality codegree (loose or strict) of a bimould is simply its alternality codegree paired with that of its swappee

$$
\operatorname{codeg}^{\operatorname{bial}}\left(A^{\bullet}\right):=\left(\operatorname{codeg}^{a l}\left(A^{\bullet}\right), \operatorname{codeg}^{a l}\left(\operatorname{swap} . A^{\bullet}\right)\right) .
$$

Ordinary bialternality corresponds to codegree $(1,1)$.

We cannot expect the bialternality codegree (or rather its second component) to behave in anything like a predictable manner under $m u$ and $l u$ nor indeed under preari and ari, but there is an important exception, namely on the subalgebra of push-invariant elements ${ }^{75}$, where swap commutes with preari and ari. So for push-invariant bimoulds we have

$$
\begin{gathered}
C^{\bullet}=\operatorname{preari}\left(A^{\bullet}, B^{\bullet}\right) \Longrightarrow \operatorname{codeg}^{\text {bial }}\left(C^{\bullet}\right)=\operatorname{codeg}^{\text {bial }}\left(A^{\bullet}\right)+\operatorname{codeg}^{\text {bial }}\left(B^{\bullet}\right), \\
C^{\bullet}=\operatorname{ari}\left(A^{\bullet}, B^{\bullet}\right) \Longrightarrow \operatorname{codeg}^{\text {bial }}\left(C^{\bullet}\right) \leq \operatorname{codeg}^{\text {bial }}\left(A^{\bullet}\right)+\operatorname{codeg}^{\text {bial }}\left(B^{\bullet}\right)-(1,1) .
\end{gathered}
$$

Here, again we have a filtration of Flex $(\mathfrak{E})$ into increasing subspaces Flex $_{r}^{\left(d_{1}, d_{2}\right)}(\mathfrak{E})$ with the corresponding dimensions

$$
\text { Bial }_{r}^{d_{1}, d_{2}}:=\operatorname{dim}\left(\text { Flex }_{r}^{\left(d_{1}, d_{2}\right)}(\mathfrak{E})\right)
$$

and the even more relevant differences

$$
\text { bial }_{r}^{d_{1}, d_{2}}:=\text { Bial }_{r}^{d_{1}, d_{2}}-\text { Bial }_{r}^{d_{1}-1, d_{2}}-\text { Bial }_{r}^{d_{1}, d_{2}-1}+\text { Bial }_{r}^{d_{1}-1, d_{2}-1}
$$

which serve as entries of the so-called bialternality grid.

In fact, we have two such grids: one for the whole of Flex $(\mathfrak{E})$ and one for the pushinvariant subalgebra Flex push $(\mathfrak{E})$. The second grid, also called bialternality chessboard, is the more important of the two, but in this "monogenous" or "eupolar" context both are

\footnotetext{
${ }^{75}$ Which, remember, contains all bialternals.
} 
equally interesting. In particular, both are symmetrical with respect to the main diagonal. This is due to the existence of a second involution syap, specific to this case.

But when we leave the "eupolar" context and move on for example to the important case of polynomial-valued bimoulds, we still have (highly interesting) bialternality grids and chessboards but there is no syap anymore and so the property of diagonal symmetry disappears, though traces of it remain.

\subsection{The Bialternality Grid for General Eupolars}

Here are the cases that proved amenable to computation:

$$
\begin{aligned}
& 3 \text { | } 100 \\
& \text { 2| } 10 \quad 2 \quad 2 \mid 110 \\
& 1\left|\frac{0}{1} \frac{1}{2} \quad 1\right| \frac{0}{1} \frac{1}{2} \frac{1}{3}
\end{aligned}
$$

\begin{tabular}{|c|c|c|c|c|c|c|}
\hline $8 \mid 1$ & 0 & 0 & 0 & 0 & 0 & 0 \\
\hline $7 \mid 7$ & 0 & 0 & 0 & 0 & 0 & 0 \\
\hline $6 \mid ?$ & $?$ & 0 & 0 & 0 & 0 & 0 \\
\hline $5 \mid ?$ & $?$ & $?$ & 0 & 0 & 0 & 0 \\
\hline 41 & $?$ & $?$ & $?$ & 0 & 0 & 0 \\
\hline 31 & $?$ & $?$ & $?$ & $?$ & 0 & 0 \\
\hline 21 & $?$ & $?$ & $?$ & $?$ & $?$ & 0 \\
\hline $1 \mid 1$ & $?$ & $?$ & $?$ & $?$ & $?$ & 7 \\
\hline & 2 & 3 & 4 & & & \\
\hline
\end{tabular}

\begin{tabular}{r|rrrrrrrrrrr} 
& & & & & 5 & 1 & 0 & 0 & 0 & 0 \\
$3 \mid$ & 1 & 0 & 0 & 0 & & $4 \mid$ & 4 & 0 & 0 & 0 & 0 \\
3 & 2 & 1 & 0 & 0 & & $3 \mid$ & 1 & 10 & 1 & 0 & 0 \\
$2 \mid$ & 0 & 5 & 1 & 0 & & $2 \mid$ & 3 & 3 & 10 & 0 & 0 \\
$1 \mid$ & 1 & 0 & 2 & 1 & & $1 \mid$ & 0 & 3 & 1 & 4 & 1 \\
& -1 & - & - & - & & & $\frac{1}{1}$ & $\frac{3}{2}$ & $\frac{1}{3}$ & $\frac{1}{4}$ & 5
\end{tabular}

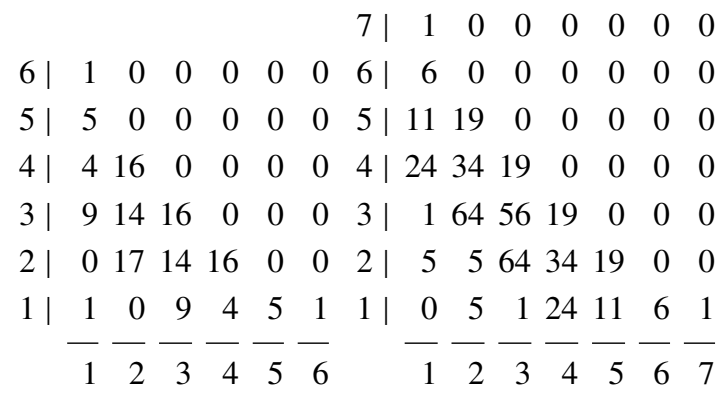

Two features stand out here: strict diagonal symmetry as well as the vanishing of all entries in the north-west triangles. Both are eupolar-specific phenomena, although as tendencies both extend, in a much weakened form, to the case of polynomial-valued bimoulds. 


\subsection{The Bialternality Chessboard for push-invariant Eupolars}

For $r<4$, all entries are 0 . For $4 \leq r \leq 8$, we get

\begin{tabular}{|c|c|}
\hline & 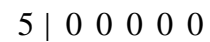 \\
\hline $4 \mid \begin{array}{llll}4 & 0 & 0 & 0\end{array}$ & $4 \mid \begin{array}{lllll}0 & 0 & 0 & 0 & 0\end{array}$ \\
\hline $3 \mid \begin{array}{llll}3 & 0 & 0 & 0\end{array}$ & $3 \mid \begin{array}{lllll}0 & 1 & 0 & 0 & 0\end{array}$ \\
\hline $2 \mid \begin{array}{llll}0 & 1 & 0 & 0\end{array}$ & $2 \mid \begin{array}{llll}1 & 0 & 1 & 0\end{array}$ \\
\hline $1 \mid \begin{array}{llll}1 & 0 & 0 & 0\end{array}$ & $1 \mid \begin{array}{llll}0 & 1 & 0 & 0\end{array}$ \\
\hline 23 & 1 \\
\hline
\end{tabular}

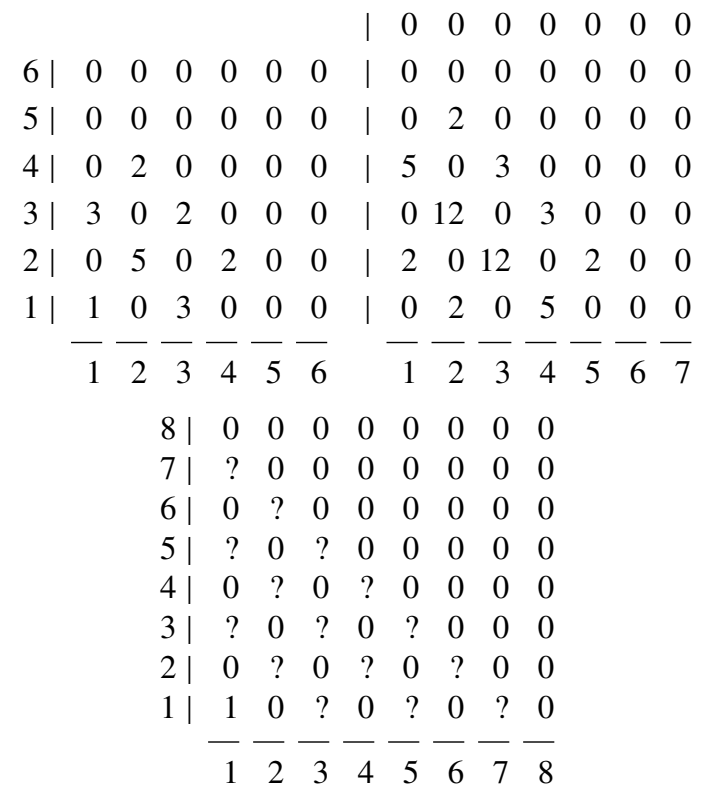

We observe the vanishing of all entries on the diagonals of equation $d_{1}-d_{2}-r=o d d$ or, what amounts to the same, on the anti-diagonals $r-d_{1}-d_{2}=o d d$. The phenomenon, this time, is not eupolar-specific but quite general and a direct consequence of push-invariance. The reasons behind it are explained in the next section, which is devoted to the case of polynomial-valued bimoulds.

Remark The case for not restricting our attention to the sole bialternals of Flex $x_{r}(\mathfrak{E})$ but rather investigating the full filtration by the bialternality codegrees, and in particular the subspaces

$$
\operatorname{flex}_{r}^{d_{1}, d_{2}}:=\operatorname{Flex}_{r}^{d_{1}, d_{2}} /\left(\operatorname{Flex}_{r}^{d_{1}-1, d_{2}}+\operatorname{Flex}_{r}^{d_{1}, d_{2}-1}\right) \quad \text { with } \quad \operatorname{dim}\left(\operatorname{flex}_{r}^{d_{1}, d_{2}}\right)=: \operatorname{bial}_{r}^{d_{1}, d_{2}}
$$

as well as their push-invariant analogues - that case is much the same as in the preceding section with the alternals. ${ }^{76}$ If anything, it is even stronger. Indeed:

(1) Each subspace flex $x_{r}^{d_{1}, d_{2}}$ is interesting in its own right.

\footnotetext{
${ }^{76}$ See the remark at the end of Section 13.
} 
(2) The simultaneous consideration of all these subspaces is often, as it was in the preceding section, a short cut to finding their individual dimensions bial $_{r}^{d_{1}, d_{2}}$ and in particular the dimension bial ${ }_{r}^{1,1}$ (if we care only about the bialternals).

(3) The grids that illustrate the filtration by the bialternality codegree, or rather the more telling gradation that goes with it, reveal two interesting symmetries: the chessboard or alternation phenomenon (for the push-invariants) and the phenomenon of the empty right upper triangle (in all grids).

\section{Introduction to the Polynomial Chessboard}

The next two sections venture beyond the eupolar into the polynomial and eutrigonometric domains, but unsystematically so, mainly with a view to showing which aspects of the eupolar situation survive and which do not. Our first prerequisite for the present survey of the polynomial case will be a series of projectors altor $r_{, j}$ that sharpen the natural filtration by the (loose) alternality codegree $j$ into a gradation by the (strict) alternality codegree; and our second prerequisite will be an $\boldsymbol{u} / \boldsymbol{v}$ exchanging involution srap capable of taking over some of the functions performed by the involution syap in the eupolar case.

\subsection{Standard Alternality Projectors (“Alternators")}

For each $j \in\{1, \ldots, r\}$, there exists a unique projector altor $_{r, j}$ that turns any $M^{\bullet} \in B I M U_{r}$ into a bimould of (strict) alternality codegree $j$ and enjoys the property that for any symmetral $S^{\bullet} \in B I M U_{r}$ the identity holds:

$$
\operatorname{altor}_{r, j} \cdot S^{\bullet} \equiv \frac{1}{j !} \operatorname{mu}_{j}\left(\operatorname{logmu} . S^{\bullet}\right) \equiv \sum_{j \leq n \leq r}(-1)^{n-j} s_{1}(n, j) \mathrm{mu}_{n}\left(S^{\bullet}\right)
$$

with $m u_{j}\left(S^{\bullet}\right)$ standing for the $j$ th $m u$-power of $S^{\bullet}$ and $s_{1}(n, j)$ denoting the (signless) Stirling numbers of the first kind

$$
x(x+1) \ldots(x+n-1) \equiv \sum_{0 \leq j \leq n} s_{1}(n, j) x^{j} .
$$

Analytically, altor $r, j$ is given as a superposition of permutators

$$
\text { (altor } \left._{r, j} \cdot M\right)^{w_{1}, \ldots, w_{r}}=\sum_{\sigma \in \mathfrak{S}_{r}} \lambda_{j}^{\sigma} \quad M^{w_{\sigma(1)}, \ldots, w_{\sigma(r)}}
$$

with coefficients $\lambda_{j}^{\sigma}=\tilde{\lambda}_{j}^{\sigma} / r !\left(\tilde{\lambda}_{j}^{\sigma} \in \mathbb{Z}\right)$ that are easily calculated by

(i) Changing $S^{\bullet}$ to $M^{\bullet}$ in (381),

(ii) Collecting all products $\prod M^{w^{i}}$ on the right-most side of (381),

(iii) Formally subjecting these products to symmetral linearisation

$$
M^{w^{1}} M^{w^{2}} \ldots M^{w^{s}} \mapsto \sum_{w \in \operatorname{sha}\left(w^{1}, w^{2}, \ldots, w^{s}\right)} M^{w}
$$

despite $M^{\bullet}$ being an arbitrary (not necessarily symmetral) bimould. 
Although these projectors altor $r, j$ are not the most "economical" as far as the number of permutators involved is concerned ${ }^{77}$, they have the advantage of being complementary

$$
\sum_{1 \leq j \leq r} \text { altor }_{r, j}=\mathrm{id}_{B I M U_{r}} \quad ; \quad \text { altor }_{r, i} \cdot \text { altor }_{r, j}=0(\forall i \neq j)
$$

and the further advantage, crucial for the sequel, of commuting not only with anti and one another, but also with the natural "projector" pushinvar $r:=\sum_{0 \leq k \leq r} p u s h^{k}$ of $B I M U_{r}$ onto the subspace of push-invariant bimoulds: ${ }^{78}$

$$
\begin{aligned}
\operatorname{altor}_{r, j} \cdot \text { anti } & =\text { anti } \cdot \operatorname{altor}_{r, j}=(-1)^{r+j} \operatorname{altor}_{r, j}, \\
\text { altor }_{r, j} \cdot \text { pushinvar }_{r} & =\operatorname{pushinvar}_{r} \cdot \text { altor }_{r, j} .
\end{aligned}
$$

We next tabulate the entire coefficients $\tilde{\lambda}_{j}^{\sigma}:=r ! \lambda_{j}^{\sigma}$ for the three cases required in the sequel, i.e., for $r \in\{3,4,5\}$. For $r=5$, we mention only the table's first half, since the rest follows under anti: see (386) supra. ${ }^{79}$

$$
\begin{array}{ccccrrrr}
\{\sigma(1) \ldots \sigma(3)\} & \tilde{\lambda}_{1}^{\sigma} & \tilde{\lambda}_{2}^{\sigma} & \tilde{\lambda}_{3}^{\sigma} & \{\sigma(1) \ldots \sigma(3)\} & \tilde{\lambda}_{1}^{\sigma} & \tilde{\lambda}_{2}^{\sigma} & \tilde{\lambda}_{3}^{\sigma} \\
\{1,2,3\} & 2 & 3 & 1 & \{2,3,1\} & -1 & 0 & 1 \\
\{1,3,2\} & -1 & 0 & 1 & \{3,1,2\} & -1 & 0 & 1 \\
\{2,1,3\} & -1 & 0 & 1 & \{3,2,1\} & 2 & -3 & 1
\end{array}
$$

$\begin{array}{lcrrrrrrrr}\{\sigma(1) \ldots \sigma(4)\} & \tilde{\lambda}_{1}^{\sigma} & \tilde{\lambda}_{2}^{\sigma} & \tilde{\lambda}_{3}^{\sigma} & \tilde{\lambda}_{4}^{\sigma} & \{\sigma(1) \ldots \sigma(4)\} & \tilde{\lambda}_{1}^{\sigma} & \tilde{\lambda}_{2}^{\sigma} & \tilde{\lambda}_{3}^{\sigma} & \tilde{\lambda}_{4}^{\sigma} \\ \{1,2,3,4\} & 6 & 11 & 6 & 1 & \{3,1,2,4\} & -2 & -1 & 2 & 1 \\ \{1,2,4,3\} & -2 & -1 & 2 & 1 & \{3,1,4,2\} & -2 & -1 & 2 & 1 \\ \{1,3,2,4\} & -2 & -1 & 2 & 1 & \{3,2,1,4\} & 2 & -1 & -2 & 1 \\ \{1,3,4,2\} & -2 & -1 & -2 & 1 & \{3,2,4,1\} & 2 & -1 & -2 & 1 \\ \{1,4,2,3\} & -2 & -1 & 2 & 1 & \{3,4,1,2\} & -2 & -1 & 2 & 1 \\ \{1,4,3,2\} & 2 & -1 & -2 & 1 & \{3,4,2,1\} & 2 & -1 & -2 & 1 \\ \{2,1,3,4\} & -2 & -1 & 2 & 1 & \{4,1,2,3\} & -2 & -1 & 2 & 1 \\ \{2,1,4,3\} & 2 & -1 & -2 & 1 & \{4,1,3,2\} & 2-1 & -2 & 1 \\ \{2,3,1,4\} & -2 & -1 & 2 & 1 & \{4,2,1,3\} & 2-1 & -2 & 1 \\ \{2,3,4,1\} & -2 & -1 & 2 & 1 & \{4,2,3,1\} & 2 & -1 & -2 & 1 \\ \{2,4,1,3\} & 2 & -1 & -2 & 1 & \{4,3,1,2\} & 2 & -1 & -2 & 1 \\ \{2,4,3,1\} & 2 & -1 & -2 & 1 & \{4,3,2,1\} & -6 & 11 & -6 & 1\end{array}$

\footnotetext{
${ }^{77}$ Thus, the most economical projectors onto the subspace of alternals involve only $2^{r-1}$ permutators.

${ }^{78}$ The true projector is of course $\frac{1}{r}$ pushinvar $_{r}$ but we dispense with the factor $\frac{1}{r}$ since it would complicate most formulae where pushinvar naturally occurs, like those in Section 10.2.

${ }^{79}$ Note that, generally speaking, $\lambda_{j}^{\sigma}$ and $\lambda_{j}^{\sigma^{-1}}$ need not coincide. So the convention adopted for denoting the permutations matters.
} 


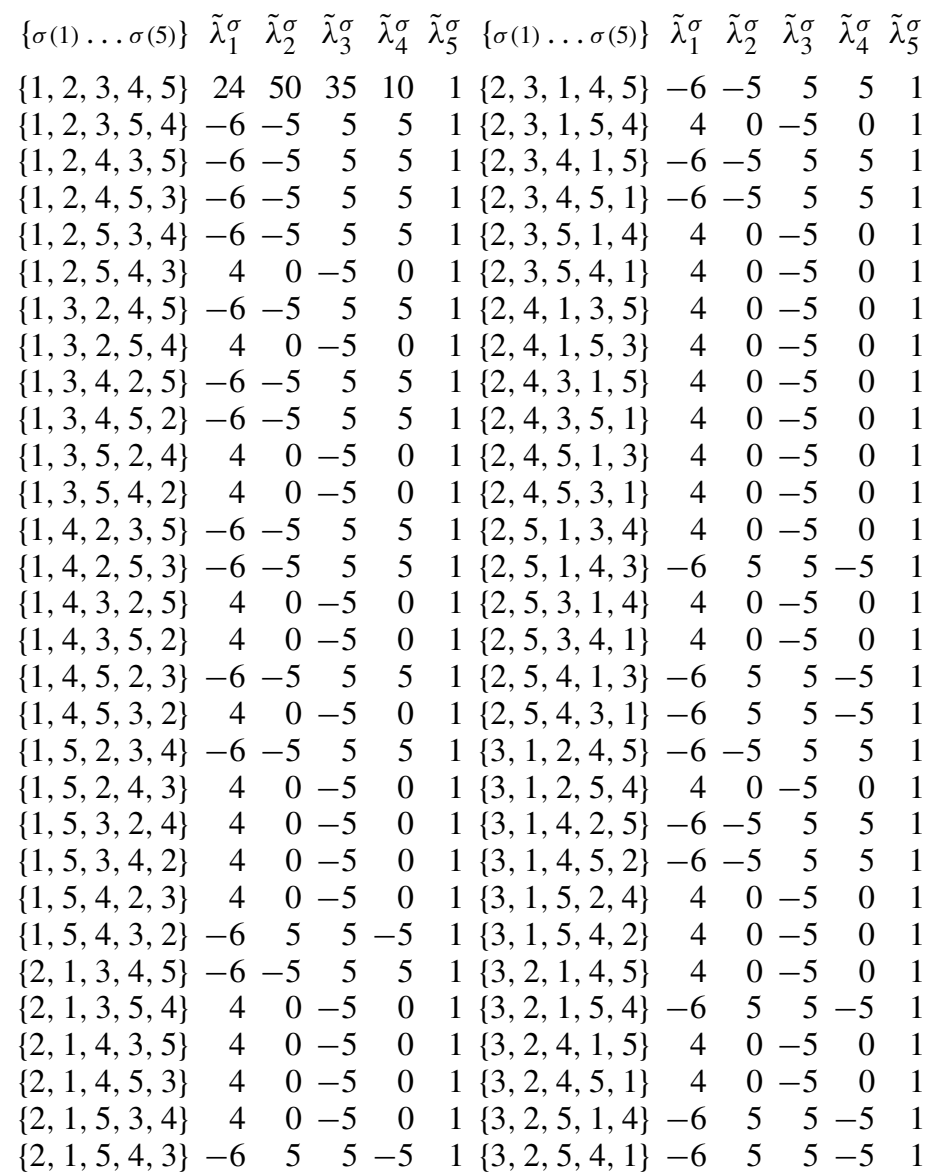

Remark 1 The dimension, noted $\tau(r+1)$ for convenience, of the subspace of permutator superpositions $\mathcal{P}$

$$
(\mathcal{P} . M)^{w_{1}, \ldots, w_{r}}=\sum_{\sigma \in \mathfrak{S}_{r}} \lambda_{j}^{\sigma} M^{w_{\sigma(1)}, \ldots, w_{\sigma(r)}} \quad\left(\mathcal{P}: \mathrm{BIMU}_{r} \rightarrow \mathrm{BIMU}_{r}\right)
$$

that commute with the "projector" pushinvar ${ }_{r}$ is of course much larger than the number $r$ of alternality projectors altor $r, j$. That dimension admits several combinatorial interpretations ${ }^{80}$ and is given by

$$
\tau(n)=\frac{1}{n^{2}} \sum_{d \mid n} \phi(d)^{2}\left(\frac{n}{d}\right) ! d^{n / d}
$$

with Euler's totient function $\phi$. The first ten values of $\tau(r+1)$ are 1, 2, 3, $8,24,108$, 640, 4492, 36336, 329900.

\footnotetext{
${ }^{80}$ The most relevant here being: the number of orbits in the set of circular permutations under cyclic permutations of the elements. See N. J. A Sloane and Simon Plouffe, A Handbook of Integer Sequences, Acad. Press, 1995.
} 
Remark 2 In the preceding sections, when dealing with the alternality grids or chessboards for eupolars, we made no use of the alternators altor $_{r, j}$ for the simple reason that these projectors do not act internally on Flex $(\mathfrak{E})$ as soon as $r \geq 4$.

\subsection{The Involution srap}

As observed in Sections 13 and 14, it is the existence of an $\boldsymbol{u} / \boldsymbol{v}$ exchanging involution syap : Flex $(\mathfrak{E}) \leftrightarrow$ Flex $_{r}(\mathfrak{D})$, respectful of the entire flexion structure and commuting with swap, that accounts for the harmony and symmetries that hold sway in the eupolar case. Unfortunately, syap does not extend beyond that setting ${ }^{81}$. For general bimoulds, we must make do with a feebler tool-the involution srap, which does not respect much of the flexion structure and fails to commute with swap, but at least preserves push-invariance and the alternality codegrees. Its action, internal on each $B I M U_{r}$, is given by the formulae

$$
\begin{aligned}
\forall \mathrm{A}^{\bullet} \in \mathrm{BIMU}_{r}, \operatorname{srap}^{\mathrm{B}_{1}, \ldots, w_{r}}=\mathrm{A}^{w_{1}^{\prime}, \ldots, w_{r}^{\prime}} & & \text { with } \\
u_{i}^{\prime}:=(r+1) v_{i}-\left(v_{1}+\cdots+v_{r}\right) & & (\forall i \in\{1, \ldots, r\}), \\
v_{i}^{\prime}:=\frac{u_{i}+\left(u_{1}+\cdots+u_{r}\right)}{r+1} & & (\forall i \in\{1, \ldots, r\}) .
\end{aligned}
$$

The above rules for the change $w_{i} \mapsto w_{i}^{\prime}$ are, needless to say, relative to the short notation, but the remarkable thing is that they extend without modification to the long notation. Indeed, if we set $u_{0}:=-u_{1} \ldots-u_{r}, v_{0}:=0$ and retain for $u_{0}^{\prime}, v_{0}^{\prime}$ the formal definition (391) and (392), we still find $u_{0}^{\prime}:=-u_{1}^{\prime} \ldots-u_{r}^{\prime}, v_{0}^{\prime}:=0$. Moreover

$$
\begin{aligned}
\text { srap } . \text { srap } & =\mathrm{id}, \\
\text { srap } \cdot \text { pushinvar } & =\text { pushinvar } . \text { srap }, \\
\text { srap } . \text { altor }_{\mathrm{j}} & =\text { altor }_{\mathrm{j}} \cdot \text { srap }
\end{aligned}
$$

These are easy identities to verify, but the main property-the preservation of pushinvariance under srap - really results from the double validity of the relations (391) and (392) which, as noted, apply equally in the short notation and in the long one. The latter, we recall, is the natural framework for the push-transform, since it reduces push to a circular permutation of order $r+1$.

\subsection{General and push-Invariant Alternality Grids}

Let $A l_{r, d}^{[j]}$, resp. $A l_{r, d}^{[[j]]}$, denote the dimension of the subspace of $B I M U_{r}$, resp. $B I M U_{r}^{\text {push }}$, consisting of bimoulds 82

(i) Constant either in all $v_{i}$ or in all $u_{i}$ variables,

(ii) Polynomial of total degree $d$ in the remaining $u_{i}$ or $v_{i}$ variables,

(iii) Of (loose) coalternality degree $j$.

Next, denote $a l_{r, d}^{[j]}:=A l_{r, d}^{[j]}-A l_{r, d}^{[j-1]}$ and $a l_{r, d}^{[[j]]}:=A l_{r, d}^{[[j]]}-A l_{r, d}^{[[j-1]]}$ the dimensions associated with the gradation induced by the alternators altor $r, j$.

Obviously, $A l_{r, d}^{[j]}$ and $a l_{r, d}^{[j]}$ do not depend on which set of variables we choose to retainwhether the $u_{i}$ 's or the $v_{i}$ 's-since the constraints of $j$-alternality are the same in both

\footnotetext{
${ }^{81}$ It does not even extend to the eutrigonmetric setting.

${ }^{82}$ As usual, BIMU $U_{r}^{\text {push }}$ denotes the push-invariant subspace of $B I M U_{r}$.
} 
cases. On the other hand, since push-invariance affects both sets of variables in quite different ways, we might expect $A l_{r, d}^{[[j]]}$ and $a l_{r, d}^{[[j]]}$ to depend on which set we retain. This is not the case, however, since in view of the relations (393), (394), (395), the evolution srap exchanges the $j$-alternal, push-invariant, $\boldsymbol{u}$-dependent bimoulds one-to-one with the $j$ alternal, push-invariant, $\boldsymbol{v}$-dependent sort. So our definitions make good sense, and we may consider the generating functions

$$
\begin{aligned}
\operatorname{ge}_{r}^{[j]}(t) & :=\sum_{0 \leq d} \mathrm{al}_{r, d}^{[j]} \cdot t^{d}, \\
\operatorname{ge}_{r}^{[[j]]}(t) & :=\sum_{0 \leq d} \mathrm{al}_{r, d}^{[[j]]} \cdot t^{d} .
\end{aligned}
$$

To understand the nature of these generating function, let $B I M U_{r}(\boldsymbol{u})$ be the space of all $\boldsymbol{u}$-polynomial, $\boldsymbol{v}$-constant bimoulds of length $r$, and consider

$$
\begin{aligned}
\operatorname{BIMU}_{r}^{[j]}(\boldsymbol{u}) & :=\operatorname{altor}_{r, j} \cdot \mathrm{BIMU}_{r}(\boldsymbol{u}), \\
\mathrm{BIMU}_{r}^{[[j]]}(\boldsymbol{u}) & :=\operatorname{pushinvar}_{r} \cdot \operatorname{altor}_{r, j} \cdot \operatorname{BIMU}_{r}(\boldsymbol{u}) .
\end{aligned}
$$

Now, the analytical constraints expressing $j$-alternality-alone or in conjunction with push-invariance-are finitary: the underlying transforms in the $\boldsymbol{u}$-variables generate a finite group. ${ }^{83}$ This circumstance makes it easy to unravel the structure of our two spaces (398) and (399) as finitely generated modules. Explicitly:

$$
\begin{aligned}
\operatorname{BIMU}_{r}^{[j]}(\boldsymbol{u}) & :=\mathrm{NU}_{r}^{[j]}(\boldsymbol{u}) \cdot \mathrm{DU}_{[r]}(\boldsymbol{u}), \\
\operatorname{BIMU}_{r}^{[[j]]}(\boldsymbol{u}) & :=\mathrm{NU}_{r}^{[[j]]}(\boldsymbol{u}) \cdot \mathrm{DU}_{[[r]]}(\boldsymbol{u}),
\end{aligned}
$$

where

(i) $\mathrm{DU}_{[r]}(\boldsymbol{u})$ denotes the ring ${ }^{84}$ of symmetric polynomials in $u_{1}, \ldots u_{r}$,

(ii) $\mathrm{DU}_{[[r]]}(\boldsymbol{u})$ denotes the ring ${ }^{85}$ of symmetric polynomials in $u_{1}, \ldots u_{r}$ and $u_{0}:=-\left(u_{1}+\right.$ $\left.\ldots+u_{r}\right)$. We may take the elementary symmetric functions of degree $2,3, \ldots, r+1$ as independent generators of $\mathrm{DU}_{[[r]]}(\boldsymbol{u})$,

(iii) $\mathrm{NU}_{r}^{[j]}(\boldsymbol{u})$ and $\mathrm{NU}_{r}^{[[j]]}(\boldsymbol{u})$ denote finite-dimensional vector spaces ${ }^{86}$ of $\boldsymbol{u}$-polynomials, with equal dimensions

$$
\operatorname{dim}\left(\mathrm{NU}_{r}^{[j]}(\mathbf{u})\right)=\operatorname{dim}\left(\mathrm{NU}_{r}^{[j]]]}(\mathbf{u})\right)=s_{1}(r, j) \quad\left(\text { with } s_{1} \text { as in (382) }\right)
$$

but with distinct sets of generators. These may be taken of the form

$$
\begin{aligned}
& \left\{\mathrm{Pa}_{d_{1}, \ldots, d_{r}}^{[j]}:=\operatorname{altor}_{r, j} \cdot \mathrm{Pa}_{d_{1}, \ldots, d_{r}}^{\bullet}\right\}, \\
& \left\{\mathrm{Pa}_{d_{1}^{\prime}, \ldots, d_{r}^{\prime}}^{[[j]]}:=\operatorname{pushinvar}_{r} \cdot \text { altor }_{r, j} \cdot \mathrm{Pa}_{d_{1}^{\prime}, \ldots, d_{r}^{\prime}}^{\bullet}\right\},
\end{aligned}
$$

\footnotetext{
${ }^{83}$ In the sense of [4], Section 2.4, p. 51.

${ }^{84}$ To make $D U_{[r]}(\boldsymbol{u})$ unitary, we add the constant polynomial 1 to its elements.

${ }^{85}$ Here again, we add 1 to $D U_{[[r]]}(\boldsymbol{u})$.

${ }^{86}$ The spaces $N U_{r}^{[j]}(\boldsymbol{u})$ and $N U_{r}^{[[j]]}(\boldsymbol{u})$ are defined only modulo multiplication by "invertible" elements of $D U_{[r]}(\boldsymbol{u})$ and $D U_{[[r]]}(\boldsymbol{u})$ respectively, in all possible ways that leave the products (400) and (401) unchanged.
} 
for two distinct sets of monomial-valued bimoulds

$$
\mathrm{Pa}_{d_{1}, \ldots, d_{r}}^{\mathbf{w}}:=u_{1}^{d_{1}} \ldots u_{r}^{d_{r}} \quad \text { and } \quad \mathrm{Pa}_{d_{1}^{\prime}, \ldots, d_{r}^{\prime}}^{\mathbf{w}}:=u_{1}^{d_{1}^{\prime}} \ldots u_{r}^{d_{r}^{\prime}} .
$$

It follows at once that our generating functions must be of the form

$$
\begin{aligned}
\operatorname{ge}_{r}^{[j]}(t) & =\mathrm{ne}_{r}^{[j]}(t) \prod_{1 \leq k \leq r}\left(1-t^{k}\right)^{-1} \quad \text { with } \quad \mathrm{ne}_{r}^{[j]}(t) \in \mathbb{N}[t], \\
\operatorname{ge}_{r}^{[[j]]}(t) & =\mathrm{ne}_{r}^{[[j]]}(t) \prod_{2 \leq k \leq r+1}\left(1-t^{k}\right)^{-1} \quad \text { with } \quad \mathrm{ne}_{r}^{[[j]]}(t) \in \mathbb{N}[t],
\end{aligned}
$$

with the shape of the numerators and denominators dictated by the nature of the spaces $N U$ and $D U$. Moreover, (402) implies

$$
\mathrm{ne}_{r}^{[j]}(1)=\mathrm{ne}_{r}^{[[j]]}(1)=s_{1}(r, j) \quad\left(s_{1} \text { as in (382) }\right) .
$$

Let $\mathcal{A}$ be the associative algebra freely generated on $\mathbb{Q}$ by $x_{1}, x_{2}$ and let $\mathcal{A}_{r, d}$ be the subspace (clearly of dimension $(r) ! /(r ! d !))$ consisting of all element of patial degrees $(r, d)$ in $\left(x_{1}, x_{2}\right)$. The coefficients $a l_{r, d}^{[j]}$ of $\mathrm{ge}_{r}^{[j]}(t)$ are easy to calculate since $a l_{r, d}^{[1]}$ (and more generally $a l_{r, d}^{[j]}$ ) can be interpreted as the dimension of the space spanned by the Lie elements in $\mathcal{A}_{r, d}$ (or more generally the elements of formal differential degree $j$ ). In particular

$$
\mathrm{al}_{r, d}^{[1]}=\frac{1}{r+d} \sum_{\delta|r, \delta| d} \mu(\delta) \frac{((r+d) / \delta) !}{(r / \delta) !((d / \delta) !} \quad(\mu=\text { Möbius function }) .
$$

Similar formulae apply for $a l_{r, d}^{[j]}$ (with $j>1$ ) and also for $a l_{r, d}^{[[j]]}$.

To sum up, in this new context of polynomial-valued bimoulds, knowing the "alternality grid" reduces to knowing the coefficients of the polynomials $n e_{r}^{[j]}(t)$ and $n e_{r}^{[[j]]}(t)$. For illustration, we tabulate infra the cases $r=3,4,5$ (the case $r=2$ being trivial). Since the polynomial $n e_{r}^{[j]}(t)$ and $n e_{r}^{[[j]]}(t)$ tend to display "higher than average" factorizability, we also give the corresponding factorizations on $\mathbb{Z}$ and $\mathbb{N}$ (the latter type being more relevant). Lastly, in the cases $r=3$, 4, we also give simple systems of generators for $\mathrm{NU}_{r}^{[j]}(\boldsymbol{u})$ and $\mathrm{NU}_{r}^{[j]]]}(\boldsymbol{u})$, in the notations (403), (404), (405).

\section{Alternality Grid for 3 Variables}

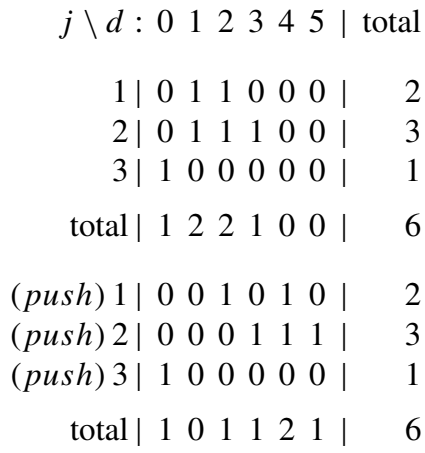


Alternality Numerators for 3 Variables

$$
\begin{aligned}
\mathrm{ne}_{3}^{[1]}(t) & =t(1+t), \\
\mathrm{ne}_{3}^{[2]}(t) & =t\left(1+t+t^{2}\right), \\
\mathrm{ne}_{3}^{[3]}(t) & =1 \\
\mathrm{ne}_{3}^{[123]}(t) & =(1+t)\left(1+t+t^{2}\right), \\
\mathrm{ne}_{3}^{[[1]]}(t) & =t^{2}\left(1+t^{2}\right), \\
\mathrm{ne}_{3}^{[[2]]}(t) & =t^{3}\left(1+t+t^{2}\right), \\
\mathrm{ne}_{3}^{[[3]]}(t) & =1, \\
\mathrm{ne}_{3}^{[[123]]}(t) & =1+t^{2}+t^{3}+2 t^{4}+t^{5}, \\
& =\left(1+t+t^{2}\right)\left(1-t+t^{2}+t^{3}\right) .
\end{aligned}
$$

\section{Alternality Generators for 3 Variables}

$$
\begin{aligned}
& \boldsymbol{d}=\mathbf{1}: \mathrm{Pa}_{100}^{[1]}, \mathrm{Pa}_{100}^{[2]} . \quad \boldsymbol{d}=\mathbf{2}: \mathrm{Pa}_{200}^{[1]}, \mathrm{Pa}_{200}^{[2]} . \quad \boldsymbol{d}=\mathbf{3}: \mathrm{Pa}_{210}^{[2]} \\
& \boldsymbol{d}=\mathbf{2}: \mathrm{Pa}_{110}^{[[1]]} . \quad \boldsymbol{d}:=\mathbf{3 :} \mathrm{Pa}_{210}^{[[2]]} . \quad \boldsymbol{d}=\mathbf{4}: \mathrm{Pa}_{211}^{[[1]]}, \mathrm{Pa}_{310}^{[[2]]} . \quad \boldsymbol{d}=\mathbf{5 :} \mathrm{Pa}_{210}^{[[2]]} .
\end{aligned}
$$

\section{Alternality Grid for 4 Variables}

$$
\begin{aligned}
& j \backslash d: 0122345678910 \mid \text { total } \\
& \text { 1| } \begin{array}{lllllllllll|r}
0 & 1 & 1 & 2 & 1 & 1 & 0 & 0 & 0 & 0 & 0 & 0
\end{array} \\
& \text { 2| } \begin{array}{lllllllllll|l}
0 & 1 & 3 & 2 & 3 & 1 & 1 & 0 & 0 & 0 & 0 & 11
\end{array} \\
& \text { 3| } \begin{array}{lllllllllll|l}
0 & 1 & 1 & 2 & 1 & 1 & 0 & 0 & 0 & 0 & 0 & \text { | }
\end{array}
\end{aligned}
$$

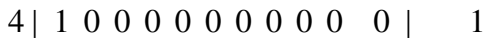

$$
\begin{aligned}
& \text { total } \mid \begin{array}{llllllllll|l}
1 & 3 & 5 & 6 & 5 & 3 & 1 & 0 & 0 & 0 & 0
\end{array} \\
& \text { (push) } 1 \mid \begin{array}{lllllllllll|l}
0 & 0 & 0 & 1 & 1 & 2 & 1 & 1 & 0 & 0 & 0 &
\end{array} \\
& \text { (push) } 2\left|\begin{array}{lllllllllll|l}
0 & 0 & 1 & 1 & 2 & 2 & 2 & 1 & 1 & 0 & 1
\end{array}\right| \begin{array}{ll}
11 \\
\text { (push) }
\end{array} \\
& \text { (push) } 3 \mid \begin{array}{lllllllllll|l}
0 & 0 & 0 & 1 & 1 & 2 & 1 & 1 & 0 & 0 & 0
\end{array} \\
& \text { (push) } 4 \mid \begin{array}{lllllllllllll}
1 & 0 & 0 & 0 & 0 & 0 & 0 & 0 & 0 & 0 & 0
\end{array} \\
& \text { total| } 1 \begin{array}{lllllllllll|l} 
& 0 & 1 & 3 & 4 & 6 & 4 & 3 & 1 & 0 & 1 & \mid
\end{array}
\end{aligned}
$$




\section{Alternality Numerators for 4 Variables}

$$
\begin{aligned}
\mathrm{ne}_{4}^{[1]}(t) & =t\left(1+t^{2}\right)\left(1+t+t^{2}\right), \\
\mathrm{ne}_{4}^{[2]}(t) & =t\left(1+3 t+2 t^{2}+3 t^{3}+t^{4}+t^{5}\right), \\
\mathrm{ne}_{4}^{[3]}(t) & =t\left(1+t^{2}\right)\left(1+t+t^{2}\right), \\
\mathrm{ne}_{4}^{[4]}(t) & =1, \\
\mathrm{ne}_{4}^{[1234]}(t) & =(1+t)^{2}\left(1+t^{2}\right)\left(1+t+t^{2}\right), \\
\mathrm{ne}_{4}^{[[1]]}(t) & =t^{3}\left(1+t^{2}\right)\left(1+t+t^{2}\right), \\
\mathrm{ne}_{4}^{[[2]]}(t) & =t^{2}\left(1+t+2 t^{2}+2 t^{3}+2 t^{4}+t^{5}+t^{6}+t^{8}\right), \\
\mathrm{ne}_{4}^{[[3]]}(t) & =t^{3}\left(1+t^{2}\right)\left(1+t+t^{2}\right), \\
\mathrm{ne}_{4}^{[[4]]}(t) & =\left(1+t^{2}\right)\left(1+3 t^{3}+4 t^{4}+3 t^{5}+t^{8}\right), \\
\mathrm{ne}_{4}^{[[1234]]}(t) & =\left(1+t^{2}\right)(1+t)^{2}\left(1+t+t^{2}\right)\left(1-3 t+5 t^{2}-3 t^{3}+t^{4}\right) .
\end{aligned}
$$

\section{Alternality Generators for 4 Variables}

$\boldsymbol{d}:=\mathbf{1}: \mathrm{Pa}_{0100}^{[1]}, \mathrm{Pa}_{0100}^{[2]}, \mathrm{Pa}_{0100}^{[3]} . \quad \boldsymbol{d}:=\mathbf{2}: \mathrm{Pa}_{0200}^{[1]}, \mathrm{Pa}_{0200}^{[2]}, \mathrm{Pa}_{1100}^{[2]}, \mathrm{Pa}_{1010}^{[[2]]}, \mathrm{Pa}_{0200}^{[3]}$. d:=3: $\mathrm{Pa}_{0300}^{[1]}, \mathrm{Pa}_{1200}^{[1]}, \mathrm{Pa}_{0300}^{[2]}, \mathrm{Pa}_{1200}^{[2]}, \mathrm{Pa}_{0300}^{[3]}, \mathrm{Pa}_{1200}^{[3]}$. d:=4: $\mathrm{Pa}_{1300}^{[1]}, \mathrm{Pa}_{1300}^{[2]}$ $\mathrm{Pa}_{1120}^{[2]}, \mathrm{Pa}_{2020}^{[2]}, \mathrm{Pa}_{1300}^{[3]}$. d:=5: $\mathrm{Pa}_{2300}^{[1]}, \mathrm{Pa}_{2300}^{[2]}, \mathrm{Pa}_{2300}^{[3]}$. d:=6: $\mathrm{Pa}_{1230}^{[2]}$.

$\boldsymbol{d}:=2: \mathrm{Pa}_{1100}^{[[1]]} . \quad \boldsymbol{d}=3: \mathrm{Pa}_{1002}^{[[1]]}, \mathrm{Pa}_{0012}^{[[2]]} . \quad \boldsymbol{d}=4: \mathrm{Pa}_{1012}^{[[1]]}, \mathrm{Pa}_{1003}^{[[2]]}, \mathrm{Pa}_{1012}^{[[2]]}, \mathrm{Pa}_{1003}^{[[3]]}$. d=5: $\mathrm{Pa}_{0113}^{[[1]]}, \mathrm{Pa}_{0122}^{[[1]]}, \mathrm{Pa}_{0113}^{[[2]]}, \mathrm{Pa}_{0122}^{[[2]]}, \mathrm{Pa}_{0113}^{[[3]]}, \mathrm{Pa}_{0122}^{[[3]]}$.

d=6: $\mathrm{Pa}_{1122}^{[[1]]}, \mathrm{Pa}_{1122}^{[[2]]}, \mathrm{Pa}_{0222}^{[[2]]}, \mathrm{Pa}_{1122}^{[[3]]}$. d=7: $\mathrm{Pa}_{1123}^{[[1]]}, \mathrm{Pa}_{1123}^{[[2]]}, \mathrm{Pa}_{1222}^{[[3]]}$.

$d=8: \mathrm{Pa}_{1123}^{[[2]]} . \quad d=10: \mathrm{Pa}_{1234}^{[[2]]}$.

\section{Alternality Grid for 5 Variables}

\begin{tabular}{rrrrrrrrrrrrrrrr|r}
$j \backslash d:$ & 1 & 1 & 2 & 3 & 4 & 5 & 6 & 7 & 8 & 9 & 10 & 11 & 12 & 13 & 14 & total \\
$1 \mid$ & 0 & 1 & 2 & 3 & 4 & 4 & 4 & 3 & 2 & 1 & 0 & 0 & 0 & 0 & 0 & 24 \\
$2 \mid$ & 0 & 1 & 3 & 6 & 8 & 10 & 9 & 7 & 4 & 2 & 0 & 0 & 0 & 0 & 0 & 50 \\
$3 \mid$ & 0 & 1 & 3 & 4 & 6 & 6 & 6 & 4 & 3 & 1 & 1 & 0 & 0 & 0 & 0 & 35 \\
$4 \mid$ & 0 & 1 & 1 & 2 & 2 & 2 & 1 & 1 & 0 & 0 & 0 & 0 & 0 & 0 & 0 & 10 \\
$5 \mid$ & 1 & 0 & 0 & 0 & 0 & 0 & 0 & 0 & 0 & 0 & 0 & 0 & 0 & 0 & 0 & 1 \\
total $\mid$ & 1 & 4 & 9 & 15 & 20 & 22 & 20 & 15 & 9 & 4 & 1 & 0 & 0 & 0 & 0 & 120 \\
(push) $1 \mid$ & 0 & 0 & 1 & 1 & 2 & 1 & 4 & 2 & 4 & 3 & 3 & 1 & 2 & 0 & 0 & 24 \\
(push) $2 \mid$ & 0 & 0 & 0 & 2 & 2 & 5 & 6 & 8 & 7 & 8 & 5 & 4 & 2 & 1 & 0 & 50 \\
(push) $3 \mid$ & 0 & 0 & 1 & 1 & 3 & 3 & 5 & 4 & 5 & 3 & 4 & 2 & 2 & 1 & 1 & 35 \\
(push) $4 \mid$ & 0 & 0 & 0 & 1 & 1 & 2 & 2 & 2 & 1 & 1 & 0 & 0 & 0 & 0 & 0 & 10 \\
(push) $5 \mid$ & 1 & 0 & 0 & 0 & 0 & 0 & 0 & 0 & 0 & 0 & 0 & 0 & 0 & 0 & 0 & 1 \\
total $\mid$ & 1 & 0 & 2 & 5 & 8 & 11 & 17 & 16 & 17 & 15 & 12 & 7 & 6 & 2 & 1 & 120
\end{tabular}




\section{Alternality Numerators for 5 Variables}

$$
\begin{aligned}
\mathrm{ne}_{5}^{[1]}(t) & =t(1+t)\left(1+t+t^{2}+t^{3}\right)\left(1+t^{2}+t^{4}\right), \\
\mathrm{ne}_{5}^{[2]}(t) & =t\left(1+t^{2}\right)\left(1+2 t+2 t^{2}\right)\left(1+t+t^{2}+t^{3}+t^{4}\right), \\
\mathrm{ne}_{5}^{[3]}(t) & =t\left(1+t+t^{2}+t^{3}+t^{4}\right)\left(1+2 t+t^{2}+2 t^{3}+t^{5}\right), \\
\mathrm{ne}_{5}^{[4]}(t) & =t\left(1+t^{2}\right)\left(1+t+t^{2}+t^{3}+t^{4}\right), \\
\mathrm{ne}_{5}^{[5]}(t) & =1, \\
\mathrm{ne}_{5}^{[1 . .5]}(t) & =(1+t)\left(1+t+t^{2}\right)\left(1+t+t^{2}+t^{3}\right)\left(1+t+t^{2}+t^{3}+t^{4}\right), \\
\mathrm{ne}_{5}^{[[1]]}(t) & =t^{2}\left(1+t^{2}\right)\left(1+t+t^{2}+3 t^{4}+2 t^{5}+t^{6}+t^{7}+2 t^{8}\right), \\
\mathrm{ne}_{5}^{[[2]]}(t) & =t^{3}\left(1+t^{2}\right)\left(1+t+t^{2}+t^{3}+t^{4}\right)\left(2+t^{2}+t^{3}+t^{4}\right), \\
\mathrm{ne}_{5}^{[[3]]}(t) & =t^{2}\left(1+t+t^{2}+t^{3}+t^{4}\right)\left(1+2 t^{2}+2 t^{4}+t^{6}+t^{8}\right), \\
\mathrm{ne}_{5}^{[[4]]}(t) & =t^{3}\left(1+t^{2}\right)\left(1+t+t^{2}+t^{3}+t^{4}\right), \\
\mathrm{ne}_{5}^{[[5]]}(t) & =1, \\
\mathrm{ne}_{5}^{[[1 . .5]]}(t) & =\left(1+t^{2}\right)\left(1+t^{2}+5 t^{3}+7 t^{4}+6 t^{5}+10 t^{6}+10 t^{7}+7 t^{8}+5 t^{9}\right. \\
& \left.+5 t^{10}+2 t^{11}+t^{12}\right) \\
& =\left(1+t^{2}\right)\left(1+t+t^{2}+t^{3}+t^{4}\right)\left(1-t+t^{2}+4 t^{3}+2 t^{4}+3 t^{6}+t^{7}+t^{8}\right) .
\end{aligned}
$$

\subsection{Bialternality Grid and Bialternality Chessboard}

Let $B i a l_{r, d}^{\left[j_{1}, j_{2}\right]}$, resp. $B i a l_{r, d}^{\left[\left[j_{1}, j_{2}\right]\right]}$, denote the dimension of the subspace $B I M U_{r, d}^{\left[j_{1}, j_{2}\right]} \subset$ $B I M U_{r}$, resp. $B I M U_{r, d}^{\left[\left[j_{1}, j_{2}\right]\right]} \subset B I M U_{r}^{\text {push }}$, consisting of all bimoulds ${ }^{87}$

(i) Constant in the $v_{i}$ variables,

(ii) Polynomial of total degree $d$ in the remaining $u_{i}$ variables,

(iii) Of (loose) alternality codegree $j_{1}$,

(iv) With a swappee of (loose) alternality codegree $j_{2}$.

Next, denote

$$
\begin{aligned}
\operatorname{bial}_{r, d}^{\left[j_{1}, j_{2}\right]} & :=B i a l_{r, d}^{\left[j_{1}, j_{2}\right]}-B_{a} l_{r, d}^{\left[j_{1}-1, j_{2}\right]}-B_{i a l}^{\left[j_{1}, j_{2}-1\right]}+B i a l_{r, d}^{\left[j_{1}-1, j_{2}-1\right]}, \\
\operatorname{bial}_{r, d}^{\left[j_{1}, j_{2}\right]} & :=B i a l_{r, d}^{\left[\left[j_{1}, j_{2}\right]\right]}-B i a l_{r, d}^{\left.\left[j_{1}-1, j_{2}\right]\right]}-B i a l_{r, d}^{\left[\left[j_{1}, j_{2}-1\right]\right]}+B i a l_{r, d}^{\left[\left[j_{1}-1, j_{2}-1\right]\right]} .
\end{aligned}
$$

The chessboard phenomenon Since the projectors altor $_{r, j_{1}}$ and swap.altor $_{r, j_{2}}$. swap do not commute, there exists no corresponding gradation by the pairs $\left[j_{1}, j_{2}\right]$ or $\left[\left[j_{1}, j_{2}\right]\right]$. In the push-invariant case, however, the filtration can be refined, leading to the vanishing of all dimensions bial ${ }_{r, d}^{\left[j_{1}, j_{2}\right]}$ when $d+j_{1}+j_{2}$ is odd.

Indeed, since push $\equiv$ neg.anti.swap.anti.swap and since neg commutes with everything, the involutions anti and swap.anti.swap, which do not commute on BIMU, do so

${ }^{87}$ As usual, $B I M U_{r}^{\text {push }}$ denotes the push-invariant subspace of $B I M U_{r}$. 
when restricted to the push-invariant subspace $B I M U^{\text {push }}$, which thus splits into a direct sum of four subspaces

$$
\mathrm{BIMU}^{\text {push }}=\bigoplus_{\epsilon_{1}, \epsilon_{1} \in\{ \pm\}} \mathcal{P}^{\epsilon_{1}, \epsilon_{2}} \cdot \mathrm{BIMU}^{\text {push }}
$$

with the projectors

$$
\mathcal{P}^{\epsilon_{1}, \epsilon_{2}}:=\frac{1}{2}\left(i d+\epsilon_{1} \text {.anti }\right) \cdot \frac{1}{2}\left(i d+\epsilon_{1} \text {.swap.anti.swap }\right)
$$

and with each of the four, $\left(\epsilon_{1}, \epsilon_{2}\right)$-indexed component spaces invariant under

$$
\epsilon_{1} \text {. anti , } \epsilon_{2} \text {. swap.anti.swap }, \epsilon_{1} . \epsilon_{2} \text {.neg. }
$$

The decomposition (410) applies in particular to $B I M U_{r, d}^{\left[\left[j_{1}, j_{2}\right]\right]}$. But in view of (386), only the component space $\mathcal{P}^{\epsilon_{1}, \epsilon_{2}} . B I M U_{r, d}^{\left[\left[j_{1}, j_{2}\right]\right]}$ with $\epsilon_{1}=(-1)^{1+j_{1}}$ and $\epsilon_{2}=(-1)^{1+j_{2}}$ may contain elements of strict bialternality codegree $\left(j_{1}, j_{2}\right)$. Moreover, due to (412), that component space has to be invariant under $\epsilon_{1} . \epsilon_{2} . n e g$ and must therefore vanish unless $d+j_{1}+j_{2}$ be even. As an immediate consequence, only the dimensions bial ${ }_{r, d}^{\left.\left[j_{1}, j_{2}\right]\right]}$ with $d+j_{1}+j_{2}$ even may be nonzero. This is the so-called chessboard phenomenon, which we had already observed in Section 14 , in the eupolar setting, where we had $d \equiv-r$ due to homogeneity.

Generating Functions As in Section 15.3, we may still form the generating series

$$
\begin{aligned}
\operatorname{gee}_{r}^{\left[j_{1}, j_{2}\right]}(t) & :=\sum_{0 \leq d} \operatorname{bial}_{r}^{\left[j_{1}, j_{2}\right]} \cdot t^{d}, \\
\operatorname{gee}_{r}^{\left[\left[j_{1}, j_{2}\right]\right]}(t) & :=\sum_{0 \leq d} \operatorname{bial}_{r}^{\left[\left[j_{1}, j_{2}\right]\right]} \cdot t^{d},
\end{aligned}
$$

but new difficulties arise, since the bialternality constraints are no longer finitary. One such difficulty is that the decompositions (400) and (401) have no equivalent here. Nonetheless, it would seem that the new generating series are still rational functions:

$$
\begin{aligned}
\operatorname{gee}_{r}^{\left[j_{1}, j_{2}\right]}(t) & =\operatorname{nee}_{r}^{\left[j_{1}, j_{2}\right]}(t) / \operatorname{dee}_{r}^{\left[j_{1}, j_{2}\right]}(t) \quad \text { with } \quad \operatorname{nee}_{r}^{\left[j_{1}, j_{2}\right]}(t), \operatorname{dee}_{r}^{\left[j_{1}, j_{2}\right]}(t) \in \mathbb{Z}[t], \\
\operatorname{gee}_{r}^{\left[\left[j_{1}, j_{2}\right]\right]}(t) & =\operatorname{nee}_{r}^{\left[\left[j_{1}, j_{2}\right]\right]}(t) / \operatorname{dee}_{r}^{\left[\left[j_{1}, j_{2}\right]\right]}(t) \quad \text { with } \quad \operatorname{nee}_{r}^{\left[\left[j_{1}, j_{2}\right]\right]}(t), \operatorname{dee}_{r}^{\left[\left[j_{1}, j_{2}\right]\right]}(t) \in \mathbb{Z}[t],
\end{aligned}
$$

with denominators dee ${ }_{r}^{\left[j_{1}, j_{2}\right]}(t)$, resp. dee $e_{r}^{\left.\left[j_{1}, j_{2}\right]\right]}(t)$, that may still ${ }^{88}$ be taken as products of elementary monomials $\left(1-t^{k}\right)$, resp. $\left(1-t^{2 k}\right)$. The numerators nee ${ }_{r}^{\left[j_{1}, j_{2}\right]}(t)$ and nee $e_{r}^{\left.\left[j_{1}, j_{2}\right]\right]}(t)$ are still polynomial in $t$, but with fairly high degrees ${ }^{89}$ and with a hopeless mixture of positive and negative (integer) coefficients. Moreover, in the push-invariant case, due to the chessboard phenomenon, nee ${ }_{r}^{\left.\left[j_{1}, j_{2}\right]\right]}(t)$ is even, resp. odd, in $t$ exactly when $j_{1}+j_{2}$ is even, resp. odd.

\footnotetext{
${ }^{88}$ Provided we do not insist on reducing the rational fonctions gee ${ }_{r}^{\left[j_{1}, j_{2}\right]}(t)$ and $\operatorname{gee}_{r}^{\left[\left[j_{1}, j_{2}\right]\right]}(t)$.

${ }^{89}$ Much higher in any case than those of the earlier ne ${ }_{r}^{[j]}(t)$ and $\mathrm{ne}_{r}^{[[j]]}(t)$.
} 
We must stress that in all generality, i.e., for all values of the length $r$, the above statements are still conjectural, unlike the corresponding results of Section 15.3 relative to the alternality grids. Another difference worth noting is the absence of bases such as (403) and (404) for the $\left[j_{1}, j_{2}\right]-$ or $\left[\left[j_{1}, j_{2}\right]\right]$-alternal subspaces, although the basis to be constructed in Section 15.5 infra may be regarded as a passable substitute.

Bialternality Grids The ordinary polynomial bialternality grids (i.e., the ones we get without imposing push-invariance) do not display the chessboard effect, nor are they symmetric under the exchange $j_{1} \leftrightarrow j_{2}$, and that too from $r=3$ onwards. Their most outstanding (still unproven) features are the vanishing of all entries $a l_{r, d}^{\left[j_{1}, j_{2}\right]}$ with $j_{1}+j_{2} \geq d+3$ (for each $r$ and $d$ large enough, i.e., $d \geq d^{*}(r)$ ) and, as already pointed out, the rationality of the generating functions $g e e_{r, d}^{\left[j_{1}, j_{2}\right]}(t)$.

Bialternality chessboards The bialternality chessboard for push-invariant bimoulds is elementary for $r=2$ and symmetric under the exchange $j_{1} \leftrightarrow j_{2}$ up to $r=4$ but not beyond ${ }^{90}$, although the deviations from symmetry remain weak even then ${ }^{91}$, much weaker at any rate than with the general grid. Moreover, the rule of the "vanishing southeast triangle" (i.e., $a l_{r, d}^{\left[j_{1}, j_{2}\right]} \equiv 0$ for $j_{1}+j_{2} \geq d+2$ and $d$ even or $j_{1}+j_{2} \geq$ $d+3$ and $d$ odd) now seems to be holding without exceptions and not jut asymptotically, as was the case with the general grid. Let us tabulate the simplest non-elementary case, i.e., $r=3$ and $r=4$.

\section{Bialternality Chessboard for Three Variables}

$$
\begin{aligned}
\operatorname{gee}_{3}^{[[1,1]]}(t) & =\frac{t^{8}+t^{10}-t^{12}}{\left(1-t^{2}\right)\left(1-t^{4}\right)\left(1-t^{6}\right)}, \\
\operatorname{gee}_{3}^{[[1,2]]}(t) & =\frac{t^{5}}{\left(1-t^{2}\right)^{2}\left(1-t^{6}\right)}, \\
\operatorname{gee}_{3}^{[[1,3]]}(t) & =\frac{t^{2}+t^{4}-t^{8}-t^{10}+t^{12}}{\left(1-t^{2}\right)\left(1-t^{4}\right)\left(1-t^{6}\right)}, \\
\operatorname{gee}_{3}^{[[2,2]]}(t) & =\frac{t^{4}}{\left(1-t^{2}\right)^{2}\left(1-t^{4}\right)}, \\
\operatorname{gee}_{3}^{[[2,3]]}(t) & =\frac{t^{3}}{\left(1-t^{2}\right)\left(1-t^{4}\right)\left(1-t^{6}\right)}, \\
\operatorname{gee}_{3}^{[[3,3]]}(t) & =1 .
\end{aligned}
$$

\footnotetext{
${ }^{90}$ This should not come as a great surprise, since the projectors altor ${ }_{r, j_{1}}$ and swap.altor $r, j_{1}$ swap do not commute on $B I M U_{r}^{\text {push }}$ any more than they do on $B I M U_{r}$. Nor does the involution srap (unlike syap in the eupolar case) exchange the bialternality types $\left[j_{1}, j_{2}\right]$ and $\left[j_{2}, j_{1}\right]$ or $\left[\left[j_{1}, j_{2}\right]\right]$ and $\left[\left[j_{2}, j_{1}\right]\right]$.

${ }^{91}$ They also appear to be limited to the case of $o d d$ degrees $d$.
} 


\section{Bialternality Chessboard for Four Variables}

$$
\begin{aligned}
\operatorname{gee}_{4}^{[[1,1]]}(t) & =\frac{t^{8}+2 t^{12}+t^{14}+t^{16}+2 t^{18}+t^{22}-t^{24}}{\left(1-t^{2}\right)\left(1-t^{6}\right)\left(1-t^{8}\right)\left(1-t^{12}\right)}, \\
\operatorname{gee}_{4}^{[[1,2]]}(t) & =\frac{t^{5}}{\left(1-t^{2}\right)^{3}\left(1-t^{6}\right)}, \\
\operatorname{gee}_{4}^{[[1,3]]}(t) & =\frac{t^{4}+3 t^{6}+6 t^{8}+11 t^{10}+14 t^{12}+17 t^{14}+17 t^{16}+15 t^{18}+11 t^{20}+7 t^{22}+4 t^{24}+t^{26}+t^{28}+t^{32}}{\left(1-t^{6}\right)\left(1-t^{8}\right)\left(1-t^{10}\right)\left(1-t^{12}\right)}, \\
\operatorname{gee}_{4}^{[[1,4]]}(t) & =\frac{t^{3}}{\left(1-t^{2}\right)^{2}\left(1-t^{6}\right)\left(1-t^{10}\right)}, \\
\operatorname{gee}_{4}^{[[2,2]]}(t) & =\frac{t^{4}+2 t^{6}+t^{8}+t^{10}-2 t^{12}+t^{14}}{\left(1-t^{2}\right)^{2}\left(1-t^{6}\right)\left(1-t^{4}\right)}, \\
\operatorname{gee}_{4}^{[[2,3]]}(t) & =\frac{t^{3}+t^{9}}{\left(1-t^{2}\right)^{3}\left(1-t^{10}\right)}, \\
\operatorname{gee}_{4}^{[[2,4]]}(t) & =\frac{t^{2}+t^{4}-t^{14}+t^{18}+t^{20}-t^{22}}{\left(1-t^{2}\right)\left(1-t^{4}\right)\left(1-t^{6}\right)\left(1-t^{10}\right)}, \\
\operatorname{gee}_{4}^{[[3,3]]}(t) & =\frac{t^{8}+2 t^{12}+t^{14}+t^{16}+2 t^{18}+t^{22}-t^{24}}{\left(1-t^{2}\right)\left(1-t^{6}\right)\left(1-t^{8}\right)\left(1-t^{12}\right)}, \\
\operatorname{gee}_{4}^{[[3,4]]}(t) & =0, \\
\operatorname{gee}_{4}^{[[4,4]]}(t) & =1 .
\end{aligned}
$$

Bialternality Chessboard for Five Variables The first (mild) deviation from symmetry occurs for degree $d=9$. Here are the corresponding entries $a l_{5,9}^{\left.\left[j_{1}, j_{2}\right]\right]}$, which duly vanish on a south-east triangle:

$$
\begin{array}{rrrrr}
0 & 8 & 0 & 14 & 0 \\
7 & 0 & 31 & 0 & 6 \\
0 & 30 & 0 & 1 & 0 \\
15 & 0 & 0 & 0 & 0 \\
0 & 6 & 0 & 0 & 0
\end{array}
$$

\subsection{Example of Bialternality Basis}

The main hurdle in the investigation of the bialternality grid and chessboard as soon as $r \geq 3$ is of course the non-finitary nature of the underlying constraints ${ }^{92}$ which precludes the existence of simple projectors and of elementary decompositions of type (400), (401). That does not make the situation totally hopeless, though, and fairly explicit bases for each type $\left[j_{1}, j_{2}\right]$ of $\left[\left[j_{1}, j_{2}\right]\right]$ may still be produced. For illustration, let us examine the simplest non-elementary case, i.e., the space of all bialternals for $r=3$ (we recall that bialternals are automatically push-invariants, as shown in Section 7 , so that the types $[1,1]$ and $[[1,1]]$ coincide; we also recall that the situation for $r=2$ is elementary since in that case the bialternality constraints are finitary, with an underlying group isomorphic with $\mathcal{S}_{3}$ ).

\footnotetext{
${ }^{92}$ This means that the analytic expression of the constraints $\left[j_{1}, j_{2}\right]$ of $\left[\left[j_{1}, j_{2}\right]\right]$ necessarily involves a set of linear transforms in the $u_{1}, v_{i}$ variables which, though finite, does generate an infinite group (when expressed, via swap, relatively to the sole variables $u_{i}$ ).
} 
We start the construction with the elementary bialternals $e k m a_{d_{1}}^{\bullet}$ for $r=1$ and $\operatorname{doma}_{d_{2}, d_{6}}^{\bullet}$ for $r=2$ :

$$
\begin{array}{rlr}
\operatorname{ekma}_{d_{1}}^{w_{1}} & :=u_{1}^{d_{1}} & \left(d_{1} \mid 2\right), \\
\operatorname{doma}_{d_{2}, d_{6}}^{w_{1}, w_{2}} & :=\mathrm{fa}\left(u_{1}, u_{2}\right) \operatorname{ha}\left(u_{1}, u_{2}\right)^{d_{2}} \mathrm{ga}\left(u_{1}, u_{2}\right)^{d_{6}} & \left(d_{2}\left|2, d_{6}\right| 6\right)
\end{array}
$$

with $^{93}$

$$
\begin{aligned}
& \operatorname{fa}\left(u_{1}, u_{2}\right):=u_{1} u_{2}\left(u_{1}-u_{2}\right)\left(u_{1}+u_{2}\right)\left(2 u_{1}+u_{2}\right)\left(2 u_{2}+u_{1}\right), \\
& \operatorname{ga}\left(u_{1}, u_{2}\right):=\left(u_{1}+u_{2}\right)^{2} u_{1}^{2} u_{2}^{2}, \\
& \operatorname{ha}\left(u_{1}, u_{2}\right):=u_{1}^{2}+u_{1} u_{2}+u_{2}^{2} .
\end{aligned}
$$

We then define length-3 bialternals tom $_{d_{1}, d_{2}, d_{6}}^{\bullet}$ as simple ari-products

$$
\text { toma }_{d_{1}, d_{2}, d_{6}}^{\bullet}:=\operatorname{ari}\left(\mathrm{ekma}_{d_{1}}^{\bullet}, \operatorname{doma}_{d_{2}, d_{6}}^{\bullet}\right) \quad\left(d_{1}\left|2, d_{2}\right| 2, d_{6} \mid 6\right) .
$$

These new bialternals are not linearly independent, since for a given total degree $d=6+$ $d_{1}+d_{2}+d_{6}$ their number exceeds that of the dimension of all length-3 bialternals. To get a basis, we must of course do more than ensure the right cardinality. Let us first consider the systems $\mathcal{B}_{d}^{0}, \mathcal{B}_{d}^{+}, \mathcal{B}_{d}^{-}$:

$$
\begin{aligned}
& \mathcal{B}_{d}^{0}:=\bigcup_{d_{2}+d_{6} \equiv 0 \bmod 4}^{d 2 \in\{0\}}\left\{\text { toma }_{d_{1}, d_{2}, d_{6}}^{\bullet}\right\}, \\
& \mathcal{B}_{d}^{+}:=\bigcup_{d_{2}+d_{6} \equiv 2 \bmod 4}^{\bigcup_{d 2 \in\{0,2\}}}\left\{\text { toma }_{d_{1}, d_{2}, d_{6}}^{\bullet}\right\}, \\
& \mathcal{B}_{d}^{-}:=\bigcup_{d_{2}+d_{6} \equiv 2 \bmod 4}^{\bigcup_{d_{1}}^{\bullet}}\left\{\text { toma }_{d_{1}, d_{2}, d_{6}}^{\bullet}\right\},
\end{aligned}
$$

with, in all three cases the common, natural conditions ${ }^{94}$

$$
d=6+d_{1}+d_{2}+d_{6} \quad ; \quad d_{1}\left|2, d_{2}\right| 2, d_{6} \mid 6 \quad ; \quad d_{1}>0, d_{2} \geq 0, d_{6} \geq 0 .
$$

Then the system $\mathcal{B}$ defined by

$$
\begin{aligned}
& \mathcal{B}_{d}:=\mathcal{B}_{d}^{0} \bigcup \mathcal{B}_{d}^{+} \quad \text { if } \quad d \equiv 0 \quad \bmod 4, \\
& \mathcal{B}_{d}:=\mathcal{B}_{d}^{0} \bigcup \mathcal{B}_{d}^{-} \quad \text { if } \quad d \equiv 2 \bmod 4
\end{aligned}
$$

has for each $d$ the right cardinality, is linearly independent, and can be shown to constitute a basis for the space of all length-3 bialternals. One way of proving this is to construct similar bases for all the other bialternality types $\left[j_{1}, j_{2}\right],\left[\left[j_{1}, j_{2}\right]\right]$ and then produce explicit, complementary projectors onto the subspaces spanned by these bases. But we are still far away from a general theory, valid for all values of $r$.

\footnotetext{
${ }^{93}$ Note that the present $\left(d_{2}, d_{6}\right)$ indexation for the doma generators slightly differs for that of (7.5) in [4], Section 7.2, p. 120.

${ }^{94}$ The first condition ensures the right degree, and the condition $d_{1}>0$ is natural, too, since $\operatorname{tom} a_{d_{1}, d_{2}, d_{6}}^{\bullet} \equiv 0$ when $d_{1}=0$.
} 


\section{From Polar to Trigonometric Bisymmetrals}

Replacing $P(t):=1 / t$ by $Q(t):=c / \tan (c t)$ changes the exact flexion units $P a^{w_{1}}:=$ $P\left(u_{1}\right)$ and $P i^{w_{1}}:=P\left(v_{1}\right)$ into the approximate units $Q a^{w_{1}}:=Q\left(u_{1}\right)$ and $Q i^{w_{1}}:=$ $Q\left(v_{1}\right)$, and turns the pair of isomorphic eupolar structures Flex $(P a)$ and Flex $(P i)$ into the non-isomorphic eutrigonometric structures Flex $(Q a)$ and Flex $(Q i)$, which remain nonisomorphic even after the (natural) extension to Flex (Qa,c.I) and Flex (Qi, c.I). These eutrigonometric structures being central to multizeta algebra ${ }^{95}$ we propose to deal with them at length in a special monograph [5], but here is a sneak preview, mainly to show which features of the eupolar case carry over and which do not.

\subsection{Disappearance of syap and Consequences}

The involution slap disappears, or rather, if we keep the formal definition of slap, loses its quality of being a full flexion isomorphism. The reason is that when we substitute $Q a$, resp. $Q i$, for $\mathfrak{E}$ in the classical three-term sum

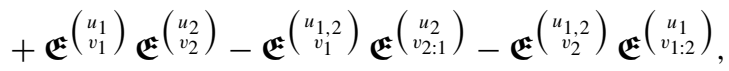

we get two constant valued elements of $B I M U_{2}$

$$
Q a a^{w_{1}, w_{2}} \equiv c^{2} \quad ; \quad Q i i^{w_{1}, w_{2}} \equiv-c^{2}
$$

instead of getting 0 , as with strict flexion units. The complication here has less to do with the sign alternation $\pm c^{2}$ than with the fact that $Q a a^{\bullet}$ ari-commutes with all elements of its parent structure Flex $(Q a)$, whereas $Q i i^{\bullet}$ does not ari-commute with Flex $(Q i)$. For instance, if we ari-bracket $\mathfrak{E}^{\bullet}$ with the length-2 bimould defined by the three-term sum (416), we get the following expression

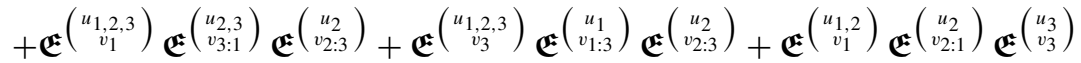

$$
\begin{aligned}
& -\mathfrak{E}^{\left(\begin{array}{c}
u_{1,2,3} \\
v_{3}
\end{array}\right)} \mathfrak{E}^{\left(\begin{array}{c}
u_{1,2} \\
v_{1: 3}
\end{array}\right)} \mathfrak{E}^{\left(\begin{array}{c}
u_{2} \\
v_{2: 1}
\end{array}\right)}-\mathfrak{E}^{\left(\begin{array}{c}
u_{1,2,3} \\
v_{1}
\end{array}\right)} \mathfrak{E}^{\left(\begin{array}{c}
u_{2} \\
v_{2}: 1
\end{array}\right)} \mathfrak{E}^{\left(\begin{array}{c}
u_{3} \\
v_{3: 1}
\end{array}\right)}-\mathfrak{E}^{\left(\begin{array}{c}
u_{2,3} \\
v_{3}
\end{array}\right)} \mathfrak{E}^{\left(\begin{array}{c}
u_{2} \\
v_{2}: 3
\end{array}\right)} \mathfrak{E}^{\left(\begin{array}{c}
u_{1} \\
v_{1}
\end{array}\right)}
\end{aligned}
$$

which vanishes under the specialization $\mathfrak{E} \mapsto Q a$ but not under $\mathfrak{E} \mapsto Q i .^{96}$

\subsection{Appearance of a Corrective "Central" Factor}

Let us now systematically contrapose the main formulae for polar bialternals and bisymmetrals to their trigonometric equivalents.

The central-exceptional bisymmetrals $\mathrm{tal}^{\bullet} / / \mathrm{ti} l^{\bullet}$ (invariant under neg.pari but neither neg nor pari ) are still exchanged by the involution swap, but only modulo gari-multiplication by an element $\operatorname{mana}^{\bullet} \in \operatorname{Centre}(G A R I)$ :

$$
\begin{aligned}
\text { swap.pil } & =\operatorname{pal}^{\bullet} \leadsto \\
\text { swap.til } & =\operatorname{gari}\left(\operatorname{tal}^{\bullet}, \operatorname{mana}^{\bullet}\right)=\operatorname{gari}\left(\operatorname{mana}{ }^{\bullet}, \operatorname{tal}^{\bullet}\right)=\operatorname{mu}\left(\operatorname{tal} l^{\bullet}, \operatorname{mana}^{\bullet}\right)
\end{aligned}
$$

with

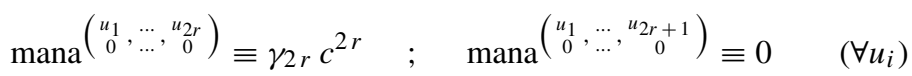

\footnotetext{
${ }^{95}$ Especially for constructing a canonical-cum-explicit and rational Drinfeld associator.

${ }^{96}$ It would vanish under the specialization $\mathfrak{E} \mapsto Q i \pm c I$ which, however, is not acceptable, since $Q i^{w^{1}} \pm$ $c I^{w_{1}}$ is not odd in $w_{1}$.
} 
and

$$
1+\sum_{1 \leq r} \gamma_{2 r} c^{2 r}=\left(\frac{\sin c}{c}\right)^{\frac{1}{2}}=1-\frac{1}{12} c^{2}+\frac{1}{1440} c^{4}+\ldots
$$

\subsection{Proliferation of Alternals and Symmetrals}

In the eupolar case, Flex $(P i)$ contains (for each $r$ and up to scalar multiplication) exactly one alternal without poles of the form $\left(v_{i}-v_{j}\right)^{-1}$ with $|i-j|>1$. By contrast, even with this restriction on the poles, Flex $r(Q i)$ contains a much richer set of alternals. The corresponding ari-structure, with its ideal of "internals" and its quotient of "externals" was investigated in [4], Section 11.5 relative to a special basis, but there exist other useful bases.

In Flex $(Q a)$, on the other hand, the most relevant alternals are those freely generated by $Q a^{\bullet}$ and $c I^{\bullet}$ under the uninflected $l u$-bracket. The corresponding algebra $L U(Q a, c I)$ belongs to the extension Flex $(Q a, c I)$ rather than Flex $(Q a)$ but the subalgebra $\mathrm{LU}^{+}(Q a, c I)$ consisting of all alternals even in $c$ is embedded in Flex $(Q a)$ itself.

\subsection{New Landscape of Bialternals and Bisymmetrals}

In the eupolar case, the involution syap, when applied to the bisymmetral pair $\mathrm{pal}^{\bullet} / / \mathrm{pil}^{\bullet}$, directly produces another central bisymmetral pair $\mathrm{par}^{\bullet} / / \mathrm{pir}^{\bullet}$ (in reverse order!) and indirectly leads to the regular (i.e., neg-invariant) bisymmetrals $\operatorname{lar}^{\bullet} / / \operatorname{lir}^{\bullet}$ and $\mathrm{ral}^{\bullet} / / \mathrm{ril}^{\bullet}$ that connect these two central pairs by gari-postcomposition. The regular bisymmetrals in turn generate a host of bialternals under logari. In the eutrigonometric setting, none of these objects survive and we are left with only one central pair $t a l^{\bullet} / / t i l^{\bullet}$

$$
\text { pal }^{\bullet} / / \text { pil }^{\bullet} \text { and } \text { par }^{\bullet} / / \text { pir }^{\bullet} \rightsquigarrow \mathrm{tal}^{\bullet} / / \mathrm{til}^{\bullet} \text { alone. }
$$

This does not mean, though, that $\operatorname{tal}^{\bullet} / / t i l^{\bullet}$ stands completely isolated. Just like pal $^{\bullet} / /$ pil $^{\bullet}$, it produces other irregular (i.e., neg.pari-invariant) bisymmetrals under postcomposition by regular (i.e., separately neg- and pari-invariant) bisymmetrals: ${ }^{97}$

$$
\begin{aligned}
& \operatorname{gari}\left(\text { pal } l^{\bullet}, \mathrm{Sa}^{\bullet}\right) / / \operatorname{gari}\left(\text { pil }^{\bullet}, \mathrm{Si}^{\bullet}\right) \rightsquigarrow \operatorname{gari}\left(\mathrm{tal} \mathbf{l}^{\bullet}, \mathrm{Za}^{\bullet}\right) / / \operatorname{gari}\left(\mathrm{til} \mathbf{l}^{\bullet}, \mathrm{Zi}^{\bullet}\right) \text { with } \\
& \text { pal }^{\bullet} / / \text { pil }^{\bullet} \text { and } \text { tal }^{\bullet} / / t i l^{\bullet} \in G A R I^{\text {as/as }} \quad \text { (neg.pari-invariant) }
\end{aligned}
$$

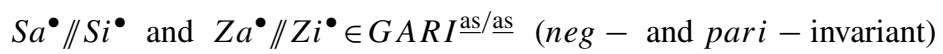

But here again, the parallelism is only approximate: there exists between the two groups of regular bisymmetrals $\mathrm{Sa}^{\bullet} / / \mathrm{Si}^{\bullet}$ and $\mathrm{Za} a^{\bullet} / / \mathrm{Zi} i^{\bullet}$ a striking disparity, which extends to the corresponding polar and trigonometric bialternals. In concrete terms:

(i) The first polar (resp. trigonometric) bialternals appear at length $r=4$ (resp. $r=8$ ).

(ii) As already noted, if we ban all poles of type $\left(v_{i}-v_{j}\right)^{-1}$ with $|i-j|>1$, we automatically ban all polar bialternals - which fact in turns leads to a neat characterization of pal ${ }^{\bullet} / /$ pil $^{\bullet}$ among all irregular bisymmetrals. On the other hand, ruling out all poles of the afore-mentioned type still leaves room for a host of trigonometric bialternals-a circumstance which makes it much harder to isolate the canonical $\mathrm{tal}^{\bullet} / / \mathrm{til}^{\bullet}$.

\footnotetext{
${ }^{97}$ Although the swappees of $\operatorname{gari}\left(\mathrm{pal}^{\bullet}, \mathrm{Sa}^{\bullet}\right)$ and $\operatorname{gari}\left(\mathrm{tal} \boldsymbol{l}^{\bullet}, \mathrm{Za^{ \bullet }}\right)$ are a priori $\operatorname{gira}\left(\mathrm{pil}^{\bullet}, \mathrm{Si} i^{\bullet}\right)$ and gira $\left(t i l^{\bullet}, Z i^{\bullet}\right)$, the latter gira-products actually coincide with gari-products since the second factors $\mathrm{Sa} a^{\bullet} / / \mathrm{Si}^{\bullet}$ and $\mathrm{Za} a^{\bullet} / / \mathrm{Zi} \bullet$ are regular bisymmetrals.
} 
(iii) Most (all?) polar bialternals seem to have no trigonometic counterpart. This applies in particular to the polar bialternals constructed in Section 9 (type I) and Section 10 (type II).

(iv) Conversely, most (all?) trigonometric bialternals seem to have no polar prototype. This applies in particular to the trigonometric bialternals of the form

$$
A^{w_{1}, \ldots, w_{2 r}}+B^{w_{1}, \ldots, w_{2 r}} Q\left(u_{1}+\ldots u_{2 r}\right) \quad(2 r \geq 8)
$$

with

$$
A^{\bullet} \in \mathrm{LU}_{2 r}^{+}(\mathrm{Q}, c . \mathrm{I}) \quad ; \quad B^{\bullet} \in \mathrm{LU}_{2 r}^{-}(\mathrm{Q}, c . \mathrm{I}) . c^{-1}
$$

which, from $2 r=8$ onwards, introduce pesky indeterminacies ${ }^{98}$ in the construction of the even factors $t a l_{e v}^{\bullet}$ and $t a l_{e v v}^{\bullet}$, to be dealt with in the next paragraph.

\subsection{New Pattern of Even-Odd Factorizations}

All three even/odd factorizations familiar from the polar case survive in the trigonometric setting, but with predictable complications: the odd factors become less elementary, while the even factors split into left and right subfactors, marked by indices lev/rev or levv/revv. Thus

$$
\begin{aligned}
& \text { pil }^{\bullet}=\operatorname{gari}\left(\text { pil }_{\text {od }}^{\bullet}, \text { pil }_{e v}^{\bullet}\right) \rightsquigarrow \\
& \mathrm{til}^{\bullet}=\operatorname{gari}\left(\mathrm{til}_{\mathrm{od}}^{\bullet}, \mathrm{til}_{e v}^{\bullet}\right) \quad \text { with } \mathrm{til}_{\mathrm{ev}}^{\bullet}=\mathrm{mu}\left(\mathrm{til}_{\mathrm{lev}}^{\bullet}, \mathrm{til}_{\mathrm{rev}}^{\bullet}\right) \quad \text { (Facto.I), } \\
& \text { pal }^{\bullet}=\operatorname{gari}\left(\text { pal }_{o d}^{\bullet}, \text { pal }_{e v}^{\bullet}\right) \rightsquigarrow \\
& \operatorname{tal}^{\bullet}=\operatorname{gari}\left(\operatorname{tal}_{\mathrm{od}}^{\bullet}, \operatorname{tal}_{e}^{\bullet}\right) \quad \text { with } \operatorname{tal}_{\mathrm{ev}}^{\bullet}=\mathrm{mu}\left(\mathrm{tal}_{\mathrm{lev}}^{\bullet}, \mathrm{tal}_{\mathrm{rev}}^{\bullet}\right) \quad(\text { Facto.II }) \text {, } \\
& \mathrm{pal}^{\bullet}=m u\left(\text { pal }_{\text {evv }}^{\bullet}, \text { pal }_{\text {odd }}^{\bullet}\right) \rightsquigarrow \\
& \text { tal }^{\bullet}=m u\left(\text { tal }_{\text {evv }}^{\bullet}, \text { tal }_{\text {odd }}^{\bullet}\right) \quad \text { with } \mathrm{tal}_{\mathrm{evv}}^{\bullet}=\mathrm{mu}\left(\mathrm{tal}_{\mathrm{levv}}^{\bullet}, \mathrm{tal}_{\mathrm{revv}}^{\bullet}\right) \quad \text { (Facto.III). }
\end{aligned}
$$

As in the polar case, this leads to a slight awkwardness (which cannot be helped) in the notations, since $t i l_{o d}^{\bullet}, t i l_{e v}^{\bullet}, t i l_{l e v}^{\bullet}, t i l_{r e v}^{\bullet}$ stand in no simple relation to tal $_{o d}^{\bullet}$, tal $_{e v}^{\bullet}$, tal $_{l e v}^{\bullet}$, tal $_{\text {rev }}^{\bullet}$ and in particular are not their swappees.

\subsection{Odd Factors: Less Elementary}

Let $\mathrm{paj}^{\bullet}, \mathrm{pij}^{\bullet}$ denote the elementary polar bimoulds defined in [4] Section 4.3 and let $\mathrm{taj}^{\bullet}$, $t i j^{\bullet}$ denote their (still reasonably elementary) trigonometric counterparts: see [4] Sections 4.3 and 4.5 .

Furthermore, for any $t \in \mathbb{Q}$ and any bimould $S^{\bullet} \in G A R I$, let $\operatorname{gari}_{t}\left(S^{\bullet}\right)$ denote the gari-iterate of order $t$ of $S^{\bullet}$

$$
\operatorname{gari}_{t}\left(S^{\bullet}\right):=\operatorname{expari}\left(t \operatorname{logari}\left(S^{\bullet}\right)\right) .
$$

The first polar-to-trigonometric transposition involves some complication

$$
\begin{aligned}
& \text { pil }_{\text {od }}^{\bullet}=\operatorname{gari}_{-\frac{1}{2}}\left(\mathrm{pij}^{\bullet}\right)=\left(-\frac{1}{2}\right)^{r(\bullet)} \mathrm{pij}^{\bullet} \rightsquigarrow \\
& \mathrm{til}_{\mathrm{od}}^{\bullet}=\text { gari }_{-\frac{1}{2}}\left(\mathrm{tij}^{\bullet}\right) \quad \neq\left(-\frac{1}{2}\right)^{r(\bullet)} \mathrm{tij}^{\bullet} .
\end{aligned}
$$

\footnotetext{
${ }^{98}$ Which, fortunately, can be removed. Even if they could not, they would be automatically offset by corrective terms in the roma $a^{\bullet}$ factor of the classical multizeta decomposition Zag ${ }^{\bullet}:=$

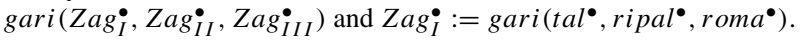


The inequality on the second line arises from the fact that, unlike in the polar case where we had an exact identity $\log \operatorname{ari}\left(\mathrm{pij}^{\bullet}\right)=P i^{\bullet}$, in the trigonometric case we only have $\operatorname{logari}\left(t i j^{\bullet}\right)=Q i^{\bullet} \bmod c^{2}$. Nonetheless, both $t i j^{\bullet}$ and gari-1 $\left(t i j^{\bullet}\right)$ possess remarkable gari-dilators whose components (barring the first one) belong to the internal ideal ARI intern.

The second transpostion is more straightforward

$$
\begin{aligned}
& \text { pal }_{\text {od }}^{\bullet}=\operatorname{gari}_{-\frac{1}{2}}\left(\mathrm{paj}^{\bullet}\right)=\left(-\frac{1}{2}\right)^{r(\bullet)} \mathrm{paj}^{\bullet} \rightsquigarrow \\
& t a l_{\mathrm{od}}^{\bullet}=\text { gari }_{-\frac{1}{2}}\left(\mathrm{taj}^{\bullet}\right)=\left(-\frac{1}{2}\right)^{r(\bullet)} \mathrm{taj}^{\bullet} \quad \text { (exactly); }
\end{aligned}
$$

the reason being that in both cases, polar and trigonometric, we now have exact identities

$$
\operatorname{logari}\left(\operatorname{paj}^{\bullet}\right)=P a^{\bullet} \quad, \quad \operatorname{logari}\left(\operatorname{taj}^{\bullet}\right)=Q a^{\bullet} .
$$

No such simplification occurs in the third transposition

$$
\begin{aligned}
\text { pal }_{\text {odd }}^{\bullet} & =\operatorname{expmu}\left(-\frac{1}{2} \mathrm{~Pa}^{\bullet}\right) \quad \rightsquigarrow \\
\operatorname{tal}_{\text {odd }}^{\bullet} & \sim \operatorname{expmu}\left(-\frac{1}{2} \mathrm{Qa}^{\bullet}+\operatorname{lutal}_{\text {odd }}^{\bullet}\right) \text { with lutal }{ }_{\text {odd }}^{\bullet} \in \mathrm{LU}^{+}(\mathrm{Qa}, c \mathrm{I})
\end{aligned}
$$

and we are saddled with a corrective alternal bimould $l u t a l_{\text {odd }}^{\bullet}$ with only (nonzero) oddlength components.

\subsection{Even Factors: Left and Right Subfactors}

The first even factor splits into a product (in both GARI and $M U$ ) of internal and external subfactors 99

$$
\begin{aligned}
\operatorname{til}_{\mathrm{ev}}^{\bullet}= & \operatorname{gari}\left(\mathrm{til}_{\mathrm{rev}}^{\bullet}, \mathrm{til}_{\mathrm{lev}}^{\bullet}\right)=\mathrm{mu}\left(\mathrm{til}_{\mathrm{lev}}^{\bullet}, \mathrm{til}_{\mathrm{rev}}^{\bullet}\right) \quad \text { (inversion!) } \\
\text { with } & \operatorname{til}_{\mathrm{lev}}^{\bullet} \in \mathrm{GARI}_{\mathrm{intern}}, \mathrm{til}_{\mathrm{rev}}^{\bullet} \in \mathrm{GARI}_{\mathrm{extern}}
\end{aligned}
$$

but the really interesting part is what happens to the second and third even factors, namely $\mathrm{tal}_{e v}^{\bullet}$ and $\mathrm{tal}_{e v v}^{\bullet}$. Surprisingly enough, both split in exactly the same way

$$
\begin{aligned}
& \operatorname{tal}_{\mathrm{lev}}^{\bullet}=\mathrm{Taj}^{\bullet} \circ \operatorname{dutal}_{\mathrm{lev}}^{\bullet} ; \quad \operatorname{der}_{\mathrm{tal}}^{\bullet} \boldsymbol{\mathrm { rev }}^{\bullet}=\mathrm{mu}\left(\mathrm{tal}_{\mathrm{rev}}^{\bullet}, \operatorname{detal}_{\mathrm{rev}}^{\bullet}\right) ; \\
& \operatorname{tal}_{\mathrm{levv}}^{\bullet}=\mathrm{Taj}^{\bullet} \circ \operatorname{dutal}_{\mathrm{levv}}^{\bullet} ; \operatorname{der}^{\bullet} \mathrm{tal}_{\mathrm{revv}}^{\bullet}=\mathrm{mu}\left(\mathrm{tal}_{\mathrm{rev}}^{\bullet}, \operatorname{detal}_{\mathrm{revv}}^{\bullet}\right)
\end{aligned}
$$

with right/left subfactors similarly related to alternals of $\mathrm{LU}^{ \pm}(\mathrm{Qa}, c . \mathrm{I})$

$$
\begin{aligned}
& \left.\operatorname{dutal}_{\mathrm{lev}}^{\bullet} \text { and } \operatorname{dutal}_{\mathrm{levv}}^{\bullet} \in \mathrm{LU}^{-}(\mathrm{Qa}), c . \mathrm{I}\right) \cdot c^{-1}, \\
& \left.\operatorname{detal}_{\mathrm{rev}}^{\bullet} \text { and } \operatorname{detal}_{\mathrm{revv}}^{\bullet} \in \mathrm{LU}^{+}(\mathrm{Qa}), c . \mathrm{I}\right) .
\end{aligned}
$$

Here, the alternals $d u t a l_{l e v}^{\bullet}, d u t a l_{l e v v}^{\bullet}$ are rough equivalents of the mu-dilators $d u p a l_{e v}^{\bullet}$, dupal $_{\text {evv }}^{\circ}$ familiar from the polar case, and the reverse passage (from the dilators to their sources) is via precomposition by the mould $\operatorname{Taj}^{\bullet}$ (form-identical with $\operatorname{taj}^{\bullet}$, but viewed as a mould rather than a bimould). As for the alternals detal $l_{\text {rev }}^{\bullet}$, detal $l_{\text {revv }}^{\bullet}$, they have no polar antecedents and are just another, particularly elementary sort of dilators. ${ }^{100}$

\footnotetext{
${ }^{99} \mathrm{Cf}$. the definitions in Sections 1-11. Note that the identity (427) (middle term = right term) looks much like the identity (55) but works for slightly different reasons, namely because $t i l_{l e v}^{\bullet}$ is internal and $t i l_{r e v}^{\bullet}$ is $\boldsymbol{u}$-constant.

${ }^{100}$ Instead of these, we might work with the slightly less simple alternals $\operatorname{logmu}\left(\right.$ tal $\left._{\text {rev }}^{\bullet}\right)$ and $\operatorname{logmu}\left(\right.$ tal $\left._{\text {revv }}^{\bullet}\right)$.
} 


\subsection{Practical Calculations}

The simplest way to calculate $\mathrm{tal}^{\bullet} / \mathrm{til}^{\bullet}$ and establish bisymmetrality is to adapt the approach of Section 4.3. But since (122) has no exact trigonometric equivalent, we must replace $d u p a l^{\bullet}$ by the $m u$-dilators of $t a l_{e v}^{\bullet}$ or $t a l_{e v v}^{\bullet}$, and dapal ${ }^{\bullet}$ by the swappee of the garidilators of $t i l^{\bullet}$ or $t i l_{e v}^{\bullet}$. So we have four options before us, all of which are practicable but none of which can be as straightforward as the polar prototype (209), not least due to the appearance, in the trigonometric case, of left and right subfactors ("lev/rev").

This is the bad news. The good news is that the mere juxtaposition of the last two factorizations "Facto. II" and "Facto. III" of Section 16.5 already leads to a set of constraints that very nearly determine $t a l^{\bullet} / t_{i} l^{\bullet}$. This is hugely helpful, since the corresponding calculations essentially take place within the uninflected algebra $L U(Q a, c . I)$. The lengthy and in places very tedious details will be set forth in [5].

\section{Basic Prerequisites}

\subsection{Elementary Flexions}

In addition to ordinary, non-commutative mould multiplication $m u($ or $\times)$

$$
A^{\bullet}=B^{\bullet} \times C^{\bullet}=\operatorname{mu}\left(B^{\bullet}, C^{\bullet}\right) \Longleftrightarrow A^{w}=\sum_{w^{1} \cdot w^{2}=w}^{r\left(w^{1}\right), r\left(w^{2}\right) \geq 0} B^{w^{1}} C^{w^{2}}
$$

and its inverse invmu

$$
(\text { invmu. } A)^{w}=\sum_{1 \leq s \leq r(w)}(-1)^{s} \sum_{w^{1} \ldots w^{s}=w} A^{w^{1}} \ldots A^{w^{s}} \quad\left(w^{i} \neq \emptyset\right)
$$

the bimoulds ${ }^{101} A^{\bullet}$ in $B I M U=\oplus_{0 \leq r} B I M U_{r}$ can be subjected to a host of specific operations, all constructed from four elementary flexions $L,\rceil,\lceil$,$\rfloor that are always defined relative$ to a given factorization of the total sequence $\boldsymbol{w}$. The way these flexions act is apparent from the following examples:

$$
\begin{aligned}
& \boldsymbol{w}=\boldsymbol{a} . \boldsymbol{b} \quad \boldsymbol{a}=\left(\begin{array}{c}
u_{1}, u_{2}, u_{3} \\
v_{1}, v_{2}, v_{3}
\end{array}\right) \quad \boldsymbol{b}=\left(\begin{array}{l}
u_{4}, u_{5}, u_{6} \\
v_{4}, v_{5}, v_{6}
\end{array}\right)
\end{aligned}
$$

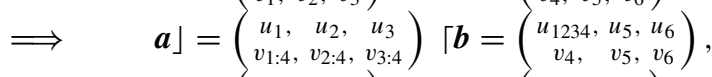

$$
\begin{aligned}
& \boldsymbol{w}=\boldsymbol{b} . \boldsymbol{c} \quad \boldsymbol{b}=\left(\begin{array}{c}
u_{1}, u_{2}, u_{3} \\
v_{1}, v_{2}, v_{3}
\end{array}\right) \quad \boldsymbol{c}=\left(\begin{array}{c}
u_{4}, u_{5}, u_{6} \\
v_{4}, v_{5}, v_{6}
\end{array}\right) \\
& \Longrightarrow \quad \boldsymbol{b}\rceil=\left(\begin{array}{l}
u_{1}, u_{2}, u_{3456} \\
v_{1}, v_{2},
\end{array} v_{3}\right) \quad\left\lfloor\boldsymbol{c}=\left(\begin{array}{ccc}
u_{4}, & u_{5}, & u_{6} \\
v_{4: 3}, & v_{5: 3}, & v_{6: 3}
\end{array}\right)\right. \text {, } \\
& \boldsymbol{w}=\boldsymbol{a} . \boldsymbol{b} . \boldsymbol{c} \boldsymbol{a}=\left(\begin{array}{c}
u_{1}, u_{2}, u_{3} \\
v_{1}, v_{2}, v_{3}
\end{array}\right) \quad \boldsymbol{b}=\left(\begin{array}{c}
u_{4}, u_{5}, u_{6} \\
v_{4}, v_{5}, v_{6}
\end{array}\right) \quad \boldsymbol{c}=\left(\begin{array}{c}
u_{7}, u_{8}, u_{9} \\
v_{7}, v_{8}, v_{9}
\end{array}\right)
\end{aligned}
$$

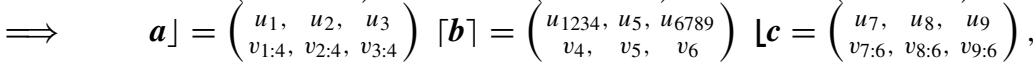

with the usual short-hand: $u_{i, \ldots, j}:=u_{i}+\ldots+u_{j}$ and $v_{i: j}:=v_{i}-v_{j}$. Here and throughout the sequel, we use boldface (with upper indexation) to denote sequences $\left(\boldsymbol{w}, \boldsymbol{w}^{i}, \boldsymbol{w}^{j}\right.$ etc), and ordinary fonts (with lower indexation) to denote single sequence elements $\left(w_{i}, w_{j}\right.$, etc.),

${ }^{101} B I M U_{r}$ of course regroups all bimoulds whose components of length other than $r$ vanish. These are often dubbed "length- $r$ bimoulds" for short. 
or sometimes sequences of length $r(\boldsymbol{w})=1$. Of course, the "product" $\boldsymbol{w}^{1} \cdot w^{2}$ denotes the concatenation of the two factor sequences.

\subsection{Short and Long Indexations on Bimoulds}

For bimoulds $M^{\bullet} \in B I M U_{r}$, it is sometimes convenient to switch from the usual short indexation (with $r$ indices $w_{i}$ 's) to a more homogeneous long indexation (with a redundant initial $w_{0}$ that gets bracketed for distinctiveness). The correspondence goes like this

$$
M^{\left(\begin{array}{c}
u_{1}, \ldots, \\
v_{1}, \ldots, \\
v_{r}
\end{array}\right)} \cong M^{\left(\begin{array}{lll}
{\left[u_{0}^{*}\right],} & u_{1}^{*}, \ldots, \\
{\left[v_{0}^{*}\right],} & v_{1}^{*}, \ldots, & v_{r}^{*}
\end{array}\right)}
$$

with the dual conditions on upper and lower indices

$$
\begin{aligned}
u_{0}^{*}=-u_{1 \ldots r}:=-\left(u_{1}+\ldots+u_{r}\right) & , u_{i}^{*}=u_{i} \forall i \geq 1, \\
v_{0}^{*} \text { arbitrary } & , v_{i}^{*}-v_{0}^{*}=v_{i} \forall i \geq 1
\end{aligned}
$$

and of course $\sum_{1 \leq i \leq r} u_{i} v_{i} \equiv \sum_{0 \leq i \leq r} u_{i}^{*} v_{i}^{*}$.

\subsection{Unary Operations}

The following linear transformations on $B I M U$ are of constant use:

$$
\begin{aligned}
& B^{\bullet}=\operatorname{minu} . A^{\bullet} \Rightarrow B^{w_{1}, \ldots, w_{r}}=-A^{w_{1}, \ldots, w_{r}}, \\
& B^{\bullet}=\text { pari. } A^{\bullet} \Rightarrow B^{w_{1}, \ldots, w_{r}}=(-1)^{r} A^{-w_{1}, \ldots,-w_{r}} \text {, } \\
& B^{\bullet}=\operatorname{anti.} A^{\bullet} \Rightarrow B^{w_{1}, \ldots, w_{r}}=A^{w_{r}, \ldots, w_{1}} \text {, } \\
& B^{\bullet}=\operatorname{mantar} . A^{\bullet} \Rightarrow B^{w_{1}, \ldots, w_{r}}=(-1)^{r-1} A^{w_{r}, \ldots, w_{1}}, \\
& B^{\bullet}=\text { neg. } A^{\bullet} \Rightarrow B^{w_{1}, \ldots, w_{r}}=A^{-w_{1}, \ldots,-w_{r}} \text {, }
\end{aligned}
$$

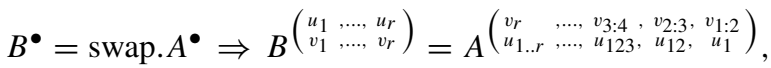

$$
\begin{aligned}
& B^{\bullet}=\operatorname{pus} . A^{\bullet} \Rightarrow B^{\left(\begin{array}{l}
u_{1}, \ldots, u_{r} \\
v_{1}, \ldots, v_{r}
\end{array}\right)}=A^{\left(\begin{array}{c}
u_{r}, u_{1}, u_{2}, \ldots, u_{r-1} \\
v_{r}, v_{1}, v_{2}, \ldots,
\end{array} v_{r-1}\right)},
\end{aligned}
$$

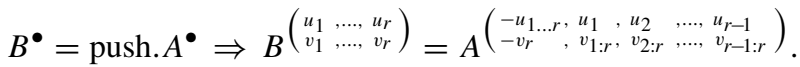

All are involutions, save for pus and push, whose restrictions to each $B I M U_{r}$ reduce to circular permutations of order $r$, resp. $r+1,{ }^{102}$

$$
\begin{aligned}
\text { push } & =\text { neg.anti.swap.anti.swap, } \\
\text { leng }_{r} & =\text { push }^{r+1} \text {.leng }_{r}=\text { pus }^{r} \text {.leng }_{r} .
\end{aligned}
$$

\subsection{Inflected Derivations and Automorphisms of $B I M U$}

Let $B I M U_{*}$, resp. $B I M U^{*}$, denote the subset of all bimoulds $M^{\bullet}$ such that $M^{\emptyset}=0$ resp. $M^{\emptyset}=1$. To each pair $\mathcal{A}^{\bullet}=\left(\mathcal{A}_{L}^{\bullet}, \mathcal{A}_{R}^{\bullet}\right) \in B I M U_{*} \times B I M U_{*}$, resp. $B I M U^{*} \times B I M U^{*}$, we attach two remarkable operators

$$
\operatorname{axit}\left(\mathcal{A}^{\bullet}\right) \in \operatorname{Der}(B I M U) \quad \operatorname{resp} . \quad \operatorname{gaxit}\left(\mathcal{A}^{\bullet}\right) \in \operatorname{Aut}(B I M U),
$$

${ }^{102}$ pus resp. push is a circular permutation in the short resp. long indexation of bimoulds. Indeed: $(\text { push } . M)^{\left[w_{0}\right], w_{1}, \ldots, w_{r}}=M^{\left[w_{r}\right], w_{0}, \ldots, w_{r-1}}$. 
whose action on $B I M U$ is given by ${ }^{103}$

$$
\begin{aligned}
N^{\bullet} & =\operatorname{axit}\left(\mathcal{A}^{\bullet}\right) . M^{\bullet} \Leftrightarrow N^{w}=\sum^{1} M^{a\lceil c} \mathcal{A}_{L}^{b\rfloor}+\sum^{2} M^{a\rfloor c} \mathcal{A}_{R}^{\lfloor b}, \\
N^{\bullet}=\operatorname{gaxit}\left(\mathcal{A}^{\bullet}\right) . M^{\bullet} \Leftrightarrow N^{w} & =\sum^{3} M^{\left\lceil b^{1}\right\rceil \ldots\left\lceil b^{s}\right\rceil} \mathcal{A}_{L}^{\left.a^{1}\right\rfloor} \ldots \mathcal{A}_{L}^{\left.a^{s}\right\rfloor} \mathcal{A}_{R}^{\left\lfloor c^{1}\right.} \ldots \mathcal{A}_{R}^{\left\lfloor c^{s}\right.}
\end{aligned}
$$

and verifies the identities

$$
\begin{aligned}
\operatorname{axit}\left(\mathcal{A}^{\bullet}\right) \cdot \operatorname{mu}\left(M_{1}^{\bullet}, M_{2}^{\bullet}\right) & \equiv \operatorname{mu}\left(\operatorname{axit}\left(\mathcal{A}^{\bullet}\right) \cdot M_{1}^{\bullet}, M_{2}^{\bullet}\right)+\operatorname{mu}\left(M_{1}^{\bullet}, \operatorname{axit}\left(\mathcal{A}^{\bullet}\right) \cdot M_{2}^{\bullet}\right), \\
\operatorname{gaxit}\left(\mathcal{A}^{\bullet}\right) \cdot \operatorname{mu}\left(M_{1}^{\bullet}, M_{2}^{\bullet}\right) & \equiv \operatorname{mu}\left(\operatorname{gaxit}\left(\mathcal{A}^{\bullet}\right) \cdot M_{1}^{\bullet}, \operatorname{gaxit}\left(\mathcal{A}^{\bullet}\right) \cdot M_{2}^{\bullet}\right) .
\end{aligned}
$$

The BIMU-derivations axit are stable under the Lie bracket for operators. More precisely, the identity holds

$$
\left[\operatorname{axit}\left(\mathcal{B}^{\bullet}\right), \operatorname{axit}\left(\mathcal{A}^{\bullet}\right)\right]=\operatorname{axit}\left(C^{\bullet}\right) \quad \text { with } \quad \mathcal{C}^{\bullet}=\operatorname{axi}\left(\mathcal{A}^{\bullet}, \mathcal{B}^{\bullet}\right)
$$

relative to a Lie law axi on $B I M U_{*} \times B I M U_{*}$ given by

$$
\begin{aligned}
& \mathcal{C}_{L}^{\bullet}:=\operatorname{axit}\left(\mathcal{B}^{\bullet}\right) \cdot \mathcal{A}_{L}^{\bullet}-\operatorname{axit}\left(\mathcal{A}^{\bullet}\right) \mathcal{B}_{L}^{\bullet}+\operatorname{lu}\left(\mathcal{A}_{L}^{\bullet}, \mathcal{B}_{L}^{\bullet}\right), \\
& \mathcal{C}_{R}^{\bullet}:=\operatorname{axit}\left(\mathcal{B}^{\bullet}\right) \cdot \mathcal{A}_{R}^{\bullet}-\operatorname{axit}\left(\mathcal{A}^{\bullet}\right) \cdot \mathcal{B}_{R}^{\bullet}-\operatorname{lu}\left(\mathcal{A}_{R}^{\bullet}, \mathcal{B}_{R}^{\bullet}\right) .
\end{aligned}
$$

Here, $l u$ denotes the standard (non-inflected) Lie law on $B I M U$

$$
\operatorname{lu}\left(A^{\bullet}, B^{\bullet}\right):=\operatorname{mu}\left(A^{\bullet}, B^{\bullet}\right)-\operatorname{mu}\left(B^{\bullet}, A^{\bullet}\right) .
$$

Let $A X I$ denote the Lie algebra consisting of all pairs $\mathcal{A}^{\bullet} \in B I M U_{*} \times B I M U_{*}$ under this law axi.

Likewise, the $B I M U$-automorphisms gaxit are stable under operator composition. More precisely

$$
\operatorname{gaxit}\left(\mathcal{B}^{\bullet}\right) \cdot \operatorname{gaxit}\left(\mathcal{A}^{\bullet}\right)=\operatorname{gaxit}\left(\mathcal{C}^{\bullet}\right) \quad \text { with } \operatorname{gaxi}\left(\mathcal{A}^{\bullet}, \mathcal{B}^{\bullet}\right)
$$

relative to a law gaxi on $B I M U^{*} \times B I M U^{*}$ given by

$$
\begin{aligned}
\mathcal{C}_{L}^{\bullet} & :=\operatorname{mu}\left(\operatorname{gaxit}\left(\mathcal{B}^{\bullet}\right) \cdot \mathcal{A}_{L}^{\bullet}, \mathcal{B}_{L}^{\bullet}\right), \\
\mathcal{A}_{R}^{\bullet} & :=\operatorname{mu}\left(\mathcal{B}_{R}^{\bullet}, \operatorname{gaxit}\left(\mathcal{B}^{\bullet}\right) \cdot \mathcal{A}_{R}^{\bullet}\right) .
\end{aligned}
$$

Let $G A X I$ denote the Lie group consisting of all pairs $\mathcal{A}^{\bullet} \in B I M U^{*} \times B I M U^{*}$ under this law gaxi.

\subsection{The Mixed Operations amnit = anmit}

For $\mathcal{A}^{\bullet}:=\left(A^{\bullet}, 0^{\bullet}\right)$ and $\mathcal{B}^{\bullet}:=\left(0^{\bullet}, B^{\bullet}\right)$ the operators axit $\left(\mathcal{A}^{\bullet}\right)$ and $\operatorname{axit}\left(\mathcal{B}^{\bullet}\right)$ reduce to $\operatorname{amit}\left(A^{\bullet}\right)$ and $\operatorname{anit}\left(B^{\bullet}\right)$ respectively, and the identity (446) becomes

$$
\operatorname{amnit}\left(A^{\bullet}, B^{\bullet}\right) \equiv \operatorname{anmit}\left(A^{\bullet}, B^{\bullet}\right) \quad\left(\forall A^{\bullet}, B^{\bullet} \in \mathrm{BIMU}_{*}\right)
$$

with

$$
\begin{aligned}
& \operatorname{amnit}\left(A^{\bullet}, B^{\bullet}\right):=\operatorname{amit}\left(A^{\bullet}\right) \cdot \operatorname{anit}\left(B^{\bullet}\right)-\operatorname{anit}\left(\operatorname{amit}\left(A^{\bullet}\right) \cdot B^{\bullet}\right), \\
& \operatorname{anmit}\left(A^{\bullet}, B^{\bullet}\right):=\operatorname{anit}\left(B^{\bullet}\right) \cdot \operatorname{amit}\left(A^{\bullet}\right)-\operatorname{amit}\left(\operatorname{anit}\left(B^{\bullet}\right) \cdot A^{\bullet}\right) .
\end{aligned}
$$

\footnotetext{
${ }^{103}$ The sum $\sum^{1}$ resp. $\sum^{2}$ extends to all sequence factorizations $\boldsymbol{w}=\boldsymbol{a} . \boldsymbol{b}$. $\boldsymbol{c}$ with $\boldsymbol{b} \neq \emptyset, \boldsymbol{c} \neq \emptyset$, resp. $\boldsymbol{a} \neq \emptyset$, $\boldsymbol{b} \neq \emptyset$. The sum $\sum^{3}$ extends to all factorizations $\boldsymbol{w}=\boldsymbol{a}^{1} \cdot b^{1} \cdot c^{1} \cdot a^{2} \cdot b^{2} \cdot c^{2} \ldots a^{s} \cdot b^{s} \cdot c^{s}$ such that $s \geq 1, \boldsymbol{b}^{i} \neq \emptyset$ $c^{i} . a^{i+1} \neq \emptyset \forall i$. Note that the extreme factor sequences $\boldsymbol{a}^{1}$ and $\boldsymbol{c}^{s}$ may be $\emptyset$.
} 
When one of the two arguments $\left(A^{\bullet}, B^{\bullet}\right)$ vanishes, the definitions reduce to

$$
\begin{gathered}
\operatorname{amnit}\left(A^{\bullet}, 0^{\bullet}\right)=\operatorname{anmit}\left(A^{\bullet}, 0^{\bullet}\right):=\operatorname{amit}\left(A^{\bullet}\right), \\
\operatorname{amnit}\left(0^{\bullet}, B^{\bullet}\right)=\operatorname{anmit}\left(0^{\bullet}, B^{\bullet}\right)=\operatorname{anit}\left(B^{\bullet}\right) .
\end{gathered}
$$

Moreover, when amnit operates on a length-1 bimould $M^{\bullet} \in B I M U_{1}$ (such as a flexion units $\mathfrak{E}^{\bullet}$, see Section 17.2 infra), its action drastically simplifies :

$$
\left.N^{\bullet}:=\operatorname{amnit}\left(A^{\bullet}, B^{\bullet}\right) \cdot M^{\bullet} \equiv \operatorname{anmit}\left(A^{\bullet}, B^{\bullet}\right) \cdot M^{\bullet} \Leftrightarrow N^{\boldsymbol{w}}=\sum_{\boldsymbol{a} w_{i} \boldsymbol{b}=\boldsymbol{w}} A^{a\rfloor} M^{\lceil} w_{i}\right\rceil B^{\lfloor\boldsymbol{b}} .
$$

\subsection{Unary Substructures}

We have two obvious subalgebras//subgroups of $A R I / / G A R I$, answering to the conditions

$$
\begin{array}{lll}
\text { AMI } \subset \text { AXI }: \mathcal{A}_{R}^{\bullet}=0^{\bullet} & , & \text { GAMI } \subset \text { GAXI }: \mathcal{A}_{R}^{\bullet}=1^{\bullet}, \\
\text { ANI } \subset \text { AXI }: \mathcal{A}_{L}^{\bullet}=0^{\bullet} & , & \text { GANI } \subset \text { GAXI }: \mathcal{A}_{L}^{\bullet}=1^{\bullet},
\end{array}
$$

but we are more interested in the mixed unary substructures, consisting of elements of the form

$$
\mathcal{A}^{\bullet}=\left(\mathcal{A}_{L}^{\bullet}, \mathcal{A}_{R}^{\bullet}\right) \quad \text { with } \quad \mathcal{A}_{R}^{\bullet} \equiv \mathrm{h}\left(\mathcal{A}_{L}^{\bullet}\right) \quad \text { and } \mathrm{h} \text { a fixed involution }
$$

with everything expressible in terms of the left element $\mathcal{A}_{L}^{\bullet}$ of the pair $\mathcal{A}^{\bullet}$. There exist, up to isomorphism, exactly seven such mixed unary substructures:

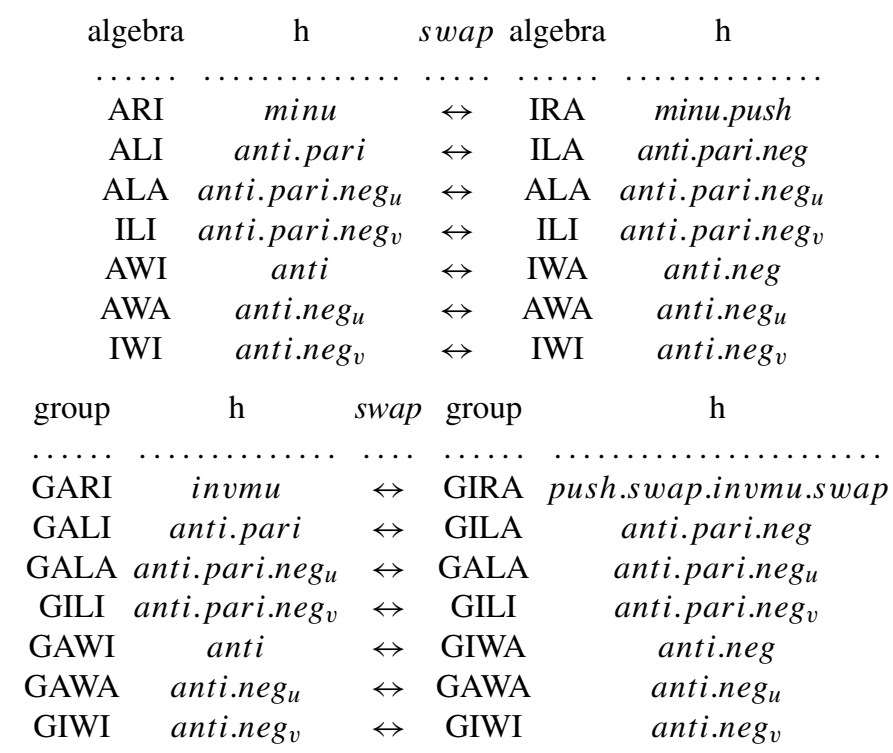

\subsection{Dimorphic Substructures}

Among all seven pairs of substructures, only two respect dimorphy, namely ARI//GARI and $A L I / / G A L I$. Moreover, when restricted to dimorphic objects, they actually coincide:

$$
\begin{aligned}
& \mathrm{ARI} \underline{\mathrm{a} l} / \underline{\mathrm{al}}=\mathrm{ALI} \underline{\mathrm{a}} / \underline{\mathrm{al}} \text { with }\{\underline{\mathrm{al}} / \underline{\mathrm{al}}\}=\{\text { alternal } / \text { alternal and even }\} \text {, } \\
& \text { GARI } \underline{\text { as }} / \underline{\text { as }}=\operatorname{GALI} \underline{\text { as }} / \underline{\text { as }} \text { with }\{\underline{\text { as }} / \underline{\text { as }}\}=\{\text { symmetral } / \text { symmetral and even }\} \text {. }
\end{aligned}
$$

We shall henceforth work with the pair ARI//GARI, whose definition involves a simpler involution $h$ (it dispenses with the sequence inversion anti: see the above table). 


\subsection{The algebra $A R I$ and its Group GARI: Basic Anti-actions}

The proper way to proceed is to define the anti-actions (on $B I M U$, with its uninflected product $m u$ and bracket $l u$ ) first of the lateral pairs $A M I / / G A M I, A N I / / G A N I$ and then of the mixed pair $A R I / / G A R I$

$$
\begin{aligned}
& N^{\bullet}=\operatorname{amit}\left(A^{\bullet}\right) \cdot M^{\bullet} \Leftrightarrow N^{w}=\sum^{1} M^{a\lceil c} A^{b\rfloor}, \\
& N^{\bullet}=\operatorname{anit}\left(A^{\bullet}\right) \cdot M^{\bullet} \Leftrightarrow N^{w}=\sum^{2} M^{a\rfloor c} A^{\lfloor b}, \\
& N^{\bullet}=\operatorname{arit}\left(A^{\bullet}\right) \cdot M^{\bullet} \Leftrightarrow N^{w}=\sum M^{a\lceil c} A^{b\rfloor}-\sum^{2} M^{a\rfloor c} A^{\lfloor b},
\end{aligned}
$$

with sums $\sum^{1}$ (resp. $\sum^{2}$ ) ranging over all sequence factorizations $\boldsymbol{w}=\boldsymbol{a} \boldsymbol{b} \boldsymbol{c}$ such that $\boldsymbol{b} \neq$ $\emptyset, \boldsymbol{c} \neq \emptyset($ resp. $\boldsymbol{a} \neq \emptyset, \boldsymbol{b} \neq \emptyset)$.

$$
\begin{aligned}
& N^{\bullet}=\operatorname{gamit}\left(A^{\bullet}\right) \cdot M^{\bullet} \Leftrightarrow N^{w}=\sum_{2}^{1} M^{\left\lceilb ^ { 1 } \ldots \left\lceil b^{s}\right.\right.} A^{\left.a^{1}\right\rfloor} \ldots A^{\left.a^{s}\right\rfloor}, \\
& N^{\bullet}=\operatorname{ganit}\left(A^{\bullet}\right) \cdot M^{\bullet} \Leftrightarrow N^{w}=\sum^{2} M^{\left.\left.b^{1}\right\rceil \ldots b^{s}\right\rceil} A^{\left\lfloor c^{1}\right.} \ldots A^{\left\lfloor c^{s}\right.}, \\
& N^{\bullet}=\operatorname{garit}\left(A^{\bullet}\right) \cdot M^{\bullet} \Leftrightarrow N^{w}=\sum^{3} M^{\left\lceil b^{1}\right\rceil \ldots\left\lceil b^{s}\right\rceil} A^{\left.a^{1}\right\rfloor} \ldots A^{\left.a^{s}\right\rfloor} A_{*}^{\left\lfloor c^{1}\right.} \ldots A_{*}^{\left\lfloor c^{s}\right.},
\end{aligned}
$$

with $A_{*}^{\bullet}:=\operatorname{invmu}\left(A^{\bullet}\right)$ and with sums $\sum^{1}, \sum^{2}, \sum^{3}$ ranging respectively over all sequence factorizations of the form

$$
\begin{aligned}
& \boldsymbol{w}=\boldsymbol{a}^{\mathbf{1}} \boldsymbol{b}^{\mathbf{1}} \ldots \boldsymbol{a}^{\boldsymbol{s}} \boldsymbol{b}^{\boldsymbol{s}} \quad\left(s \geq 1, \text { only } \boldsymbol{a}^{\mathbf{1}} \text { may be } \emptyset\right), \\
& \boldsymbol{w}=b^{\mathbf{1}} c^{1} \ldots b^{s} c^{s} \quad\left(s \geq 1 \text {, only } c^{s} \text { may be } \emptyset\right), \\
& w=a^{1} b^{1} c^{1} \ldots a^{s} b^{s} c^{s}\left(s \geq 1 \text {, with } b^{i} \neq \emptyset \text { and } c^{i} a^{i+1} \neq \emptyset\right) \text {. }
\end{aligned}
$$

More precisely, in $\sum^{3}$, two inner neighbor factors $\boldsymbol{c}^{\boldsymbol{i}}$ and $\boldsymbol{a}^{\boldsymbol{i}+\mathbf{1}}$ may vanish separately but not simultaneously, whereas the outer factors $\boldsymbol{a}^{\mathbf{1}}$ and $\boldsymbol{c}^{\boldsymbol{s}}$ may of course vanish separately or even simultaneously.

\subsection{The Algebra $A R I$ and Its Group GARI: Lie Brackets and Group Laws}

We can now concisely express the Lie brackets ami, ani, ari, and the group products gami, gani, gari :

$$
\begin{aligned}
\operatorname{ami}\left(A^{\bullet}, B^{\bullet}\right) & :=\operatorname{amit}\left(B^{\bullet}\right) \cdot A^{\bullet}-\operatorname{amit}\left(A^{\bullet}\right) \cdot B^{\bullet}+\operatorname{lu}\left(A^{\bullet}, B^{\bullet}\right), \\
\operatorname{ani}\left(A^{\bullet}, B^{\bullet}\right) & :=\operatorname{anit}\left(B^{\bullet}\right) \cdot A^{\bullet}-\operatorname{anit}\left(A^{\bullet}\right) \cdot B^{\bullet}-\operatorname{lu}\left(A^{\bullet}, B^{\bullet}\right), \\
\operatorname{ari}\left(A^{\bullet}, B^{\bullet}\right) & :=\operatorname{arit}\left(B^{\bullet}\right) \cdot A^{\bullet}-\operatorname{arit}\left(A^{\bullet}\right) \cdot B^{\bullet}+\operatorname{lu}\left(A^{\bullet}, B^{\bullet}\right), \\
\operatorname{gami}\left(A^{\bullet}, B^{\bullet}\right) & \left.:=\operatorname{mu}\left(\operatorname{gamit}\left(B^{\bullet}\right) \cdot A^{\bullet}\right), B^{\bullet}\right), \\
\operatorname{gani}\left(A^{\bullet}, B^{\bullet}\right) & \left.:=\operatorname{mu}\left(B^{\bullet}, \operatorname{ganit}\left(B^{\bullet}\right) \cdot A^{\bullet}\right)\right), \\
\operatorname{gari}\left(A^{\bullet}, B^{\bullet}\right) & \left.:=\operatorname{mu}\left(\operatorname{garit}\left(B^{\bullet}\right) \cdot A^{\bullet}\right), B^{\bullet}\right) .
\end{aligned}
$$




\subsection{The Algebra $A R I$ and Its Group GARI: Pre-lie Brackets}

Parallel with the three Lie brackets, we have three pre-Lie brackets

$$
\begin{aligned}
\operatorname{preami}\left(A^{\bullet}, B^{\bullet}\right) & :=\operatorname{amit}\left(B^{\bullet}\right) \cdot A^{\bullet}+\operatorname{mu}\left(A^{\bullet}, B^{\bullet}\right), \\
\operatorname{preani}\left(A^{\bullet}, B^{\bullet}\right) & :=\operatorname{anit}\left(B^{\bullet}\right) \cdot A^{\bullet}-\operatorname{mu}\left(A^{\bullet}, B^{\bullet}\right) \quad(\operatorname{sign} !), \\
\operatorname{preari}\left(A^{\bullet}, B^{\bullet}\right) & :=\operatorname{arit}\left(B^{\bullet}\right) \cdot A^{\bullet}+\operatorname{mu}\left(A^{\bullet}, B^{\bullet}\right),
\end{aligned}
$$

with the usual relations

$$
\begin{aligned}
\operatorname{ari}\left(A^{\bullet}, B^{\bullet}\right) & \equiv \operatorname{preari}\left(A^{\bullet}, B^{\bullet}\right)-\operatorname{preari}\left(B^{\bullet}, A^{\bullet}\right), \\
\operatorname{assopreari}\left(A^{\bullet}, B^{\bullet}, C^{\bullet}\right) & \equiv \operatorname{assopreari}\left(A^{\bullet}, C^{\bullet}, B^{\bullet}\right),
\end{aligned}
$$

with assopreari denoting the associator of the pre-Lie bracket preari. The same holds of course for ami and ani.

\subsection{Exponentiation from $A R I$ to $G A R I$}

Provided we properly define the multiple pre-Lie brackets, i.e., from left to right

$$
\text { preari }\left(A_{1}^{\bullet}, \ldots, A_{s}^{\bullet}\right)=\operatorname{preari}\left(\operatorname{preari}\left(A_{1}^{\bullet}, \ldots, A_{s-1}^{\bullet}\right), A_{s}^{\bullet}\right),
$$

we have a simple expression for the exponential mapping from a Lie algebra to its group. Thus, the exponential expari $: A R I \rightarrow G A R I$ can be expressed as a series of pre-brackets:

$$
\operatorname{expari}\left(A^{\bullet}\right)=\sum_{n} \frac{1}{n !} \operatorname{preari}(\overbrace{A^{\bullet}, \ldots, A^{\bullet}}^{n \text { times }}) \text {. }
$$

\subsection{Flexion Units}

A flexion unit $\mathfrak{E}$ is an element of $B I M U_{1}$ that verifies identically

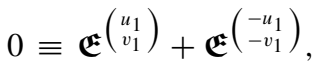

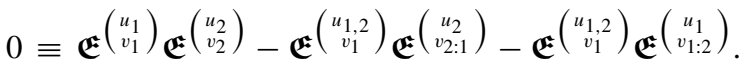

The above identities may be rewritten as

$$
0 \equiv\left(\sum_{0 \leq n<r} \operatorname{push}^{n}\right) \operatorname{mu}(\overbrace{\mathfrak{E}^{\bullet}, \ldots, \mathfrak{E}^{\bullet}}^{\text {rtimes }})
$$

for $r=1$ and 2, but they actually imply (481) for all values of $r$.

The present paper deals mainly with the polar units $\mathrm{Pa}, \mathrm{Pi}$ :

$$
\mathrm{Pa}^{w_{1}}:=P\left(u_{1}\right)=\frac{1}{u_{1}} \quad, \quad \mathrm{Pi}^{w_{1}}:=P\left(v_{1}\right)=\frac{1}{v_{1}}
$$

and occasionally with the approximate trigonometric units $Q a, Q i$ :

$$
\mathrm{Qa}^{w_{1}}:=Q\left(u_{1}\right)=\frac{c}{\tan \left(c u_{1}\right)} \quad, \quad \mathrm{Qi}^{w_{1}}:=Q\left(v_{1}\right)=\frac{c}{\tan \left(c v_{1}\right)}
$$

for which the expression on the right hand side of (480), instead of vanishing, becomes $\pm c^{2}$.

For a more substantive exposition of the flexion structure, we refer to [3] and [4]. 


\section{Tables and Maple Programs}

\subsection{A Maple Programs}

\subsubsection{Standard Eupolar Bases}

The following commands sekatal(r), sekitil(r), seketel(r) calculate the standard bases respectively of Flex $_{r}(\mathrm{~Pa})$, Flex $_{r}(\mathrm{Pi})$, Flex $_{r}(\mathrm{E})$, with $\mathrm{E}$ standing for a general flexion unit $\mathfrak{E}^{\bullet}$.

kat: $=\mathrm{n} \rightarrow(2 * n) ! / n ! /(n+1) !:$

faa $:=\operatorname{proc}(\mathrm{p}, \mathrm{q}): \operatorname{proc}(\mathrm{X})$ :

$\operatorname{subs}(\operatorname{seq}(\mathrm{u}\|(\mathrm{q}+1-\mathrm{k})=\mathrm{u}\|(\mathrm{q}+1-\mathrm{k}+\mathrm{p}), \mathrm{k}=1 . . \mathrm{q}), \mathrm{X})$ end: end:

fii: $=\operatorname{proc}(p, q): \operatorname{proc}(X): \operatorname{subs}(\operatorname{seq}(v\|(q+1-k)=v\|(q+1-k+p), k=1 . . q)$,

$\operatorname{seq}(v\|k=v\| k-v \| p, k=p+1 . . p+q), X)$ end: end:

fee:=proc $(p, q): \operatorname{proc}(X): \operatorname{fii}(p, q)(f a a(p, q)(X))$ end: end:

gaa: $=\operatorname{proc}(\mathrm{p}, \mathrm{q}): \operatorname{proc}(\mathrm{X}): \mathrm{X}$ end: end:

gii:=proc(p,q): $\operatorname{proc}(X)$ : $\operatorname{subs}(\operatorname{seq}(v\|k=v\| k-v \| p, k=1 . . p-1), X)$ end: end:

gee:=gii:

Faa: $=\operatorname{proc}(p, q): \operatorname{proc}(S):[\operatorname{seq}(\mathbf{f a a}(\mathrm{p}, \mathrm{q})(\mathrm{op}(\mathrm{s}, \mathrm{S})), \mathrm{s}=1 . . \operatorname{nops}(\mathrm{S}))]$ end: end:

Gaa: $=\operatorname{proc}(p, q): \operatorname{proc}(S):[\operatorname{seq}(\operatorname{gaa}(p, q)(o p(s, S)), s=1 . . \operatorname{nops}(S))]$ end: end:

Fii:=proc(p,q): $\operatorname{proc}(S)$ : $[\operatorname{seq}(\operatorname{fii}(\mathrm{p}, \mathrm{q})(\mathrm{op}(\mathrm{s}, \mathrm{S})), \mathrm{s}=1 . . \operatorname{nops}(\mathrm{S}))]$ end: end:

Gii:=proc $(p, q): \operatorname{proc}(S):[\operatorname{seq}(\operatorname{gii}(p, q)(o p(s, S)), s=1 . . n o p s(S))]$ end: end:

Fee:=proc(p,q): $\operatorname{proc}(S):[\operatorname{seq}(f e e(p, q)(o p(s, S)), s=1 . . n o p s(S))]$ end: end:

Gee:=proc(p,q): $\operatorname{proc}(S)$ : $[\operatorname{seq}(\operatorname{gee}(p, q)(o p(s, S)), s=1 . . \operatorname{nops}(S))]$ end: end:

Gluu:=proc(S1,S2,S3): seq(seq(op(s1,S1)*op(s2,S2)*S3,

$\mathrm{s} 1=1 . . \operatorname{nops}(\mathrm{S} 1)), \mathrm{s} 2=1 . . \operatorname{nops}(\mathrm{S} 2))$ end:

kaa: $=\operatorname{proc}(\mathrm{p}, \mathrm{q}): \mathrm{P}(\operatorname{add}(\mathrm{u} \| \mathrm{k}, \mathrm{k}=1 . . \mathrm{p}+\mathrm{q}))$ end:

kii :=proc $(\mathrm{p}, \mathrm{q}): \mathrm{P}(\mathrm{v} \| \mathrm{p})$ end:

kee: $=\operatorname{proc}(p, q): E(\operatorname{add}(u \| k, k=1 . . p+q))(v \| p)$ end:

sekatal:=proc(r) option remember; if $r=0$ then [1] elif $r=1$ then $[P(u 1)]$ else $[\operatorname{seq}($

$\operatorname{Gluu}(\operatorname{Gaa}(\mathrm{r}-\mathrm{k}, \mathrm{k})(\operatorname{sekatal}(\mathrm{r}-1-\mathrm{k})), \boldsymbol{F a a}(\mathrm{r}-\mathrm{k}, \mathrm{k})(\operatorname{sekatal}(\mathrm{k})), \mathbf{k a a}(\mathrm{r}-\mathrm{k}, \mathrm{k}))$,

$\mathrm{k}=0 . . \mathrm{r}-1)]$ fi end:

sekitil:=proc(r) option remember; if $r=0$ then [1] elif $r=1$ then $[P(v 1)]$ else $[\operatorname{seq}($

Gluu(Gii(r-k,k)(sekitil(r-1-k)),Fii(r-k,k)(sekitil(k)),kii(r-k,k)),

$\mathrm{k}=0 . . \mathrm{r}-1)$ ] fi end:

seketel:=proc(r) option remember; if $r=0$ then [1] elif $r=1$ then $[\mathrm{E}(\mathrm{u} 1)(\mathrm{v} 1)]$ else [seq(

$\operatorname{Gluu}(\operatorname{Gee}(\mathrm{r}-\mathrm{k}, \mathrm{k})(\operatorname{seketel}(\mathrm{r}-1-\mathrm{k})), \boldsymbol{F e e}(\mathrm{r}-\mathrm{k}, \mathrm{k})($ seketel $(\mathrm{k})), \mathbf{k e e}(\mathrm{r}-\mathrm{k}, \mathrm{k}))$,

$\mathrm{k}=0 . . \mathrm{r}-1)$ ] fi end: 


\subsubsection{Standard Eupolar Projectors}

kat: $=\mathrm{n} \rightarrow(2 * \mathrm{n}) ! / \mathrm{n} ! /(\mathrm{n}+1) !:$

fe: $=\operatorname{proc}(n): \operatorname{proc}(X):[\operatorname{seq}(n+o p(k, X), k=1 . . n o p s(X))]$ end: end:

$\mathbf{F e}:=\operatorname{proc}(\mathrm{n}): \operatorname{proc}(\mathrm{XX}):[\operatorname{seq}(\mathrm{fe}(\mathrm{n})(\mathrm{op}(\mathrm{kk}, \mathrm{XX})), \mathrm{kk}=1 . . n o p s(\mathrm{XX}))]$ end: end:

glu:=proc(X1,X2,X3):[op(X1),op(X2),op(X3)] end:

Glu:=proc(S1,S2,S3):seq(seq(glu(op(kk1,S1),op(kk2,S2),X3),

$\mathrm{s} 1=1 . . \operatorname{nops}(\mathrm{S} 1)), \mathrm{s} 2=1 . . \operatorname{nops}(\mathrm{S} 2))$ end:

sekat:=proc(r) option remember; if $\mathrm{r}=0$ then [[]] elif $\mathrm{r}=1$ then [[1]] else

$[\operatorname{seq}(\operatorname{Glu}(\operatorname{sekat}(r-1-k), \mathbf{F e}(r-k)(\operatorname{sekat}(k)),[r-k]), k=0 . . r-1)]$ fi end:

kow:=proc(x): $\operatorname{proc}(X): \operatorname{subs}(x=0, \operatorname{coeff}(X, P(x))-\operatorname{coeff}(X, P(-x)))$ end: end:

koka:=proc $(\mathrm{r}): \operatorname{proc}(\mathrm{m}): \operatorname{proc}(\mathrm{K})$ option remember;

if $\mathrm{r}=1$ then $\operatorname{kow}(\mathrm{u} \|(\mathrm{op}(-1, \mathrm{op}(\mathrm{m}, \mathrm{K}))))$

elif $r>1$ then $\operatorname{koka}(r-1)(m)(K) @ \operatorname{kow}(u \|(o p(-r, o p(m, K))))$ fi end: end: end:

koki: $=\operatorname{proc}(\mathrm{r})$ : $\operatorname{proc}(\mathrm{m})$ : $\operatorname{proc}(\mathrm{K})$ option remember;

if $\mathrm{r}=1$ then $\operatorname{kow}(\mathrm{v} \|(\mathrm{op}(1, \mathrm{op}(\mathrm{m}, \mathrm{K}))))$

elif $r>1$ then $\operatorname{koki}(r-1)(m)(K) @ \operatorname{kow}(v \|(o p(r, o p(m, K))))$ fi end: end: end:

kokata:=proc $(\mathrm{r}, \mathrm{m})$ option remember; $\mathbf{k o k a}(\mathrm{r})(\mathrm{m})(\operatorname{sekat}(\mathrm{r}))$ end:

kokiti:=proc(r,m) option remember; $\boldsymbol{k o k i}(\mathrm{r})(\mathrm{m})(\operatorname{sekat}(\mathrm{r}))$ end:

vokata: $=\operatorname{proc}(\mathrm{r}): \operatorname{proc}(\mathrm{X})$ option remember;

$[\operatorname{seq}(\operatorname{kokata}(\mathrm{r}, \mathrm{m})(X), \mathrm{m}=1 . . \operatorname{kat}(r))]$ end: end:

vokiti: $=\operatorname{proc}(\mathrm{r}): \operatorname{proc}(\mathrm{X})$ option remember;

$[\operatorname{seq}(\operatorname{kokiti}(\mathrm{r}, \mathrm{m})(X), \mathrm{m}=1 . . \operatorname{kat}(r))]$ end: end:

\# Comment: vokata(r)(X), resp. vokata $(\mathrm{r})(\mathrm{X})$, projects any length- $r$ eupolar $\mathrm{X}$, whatever its expression, onto the standard basis of $\operatorname{Flex}_{r}(\mathrm{~Pa})$. resp. Flex $\left._{r}(\mathrm{~Pa})\right)$.

\subsubsection{Computation of the slant-Coefficients for $\left\{\mathfrak{h} \mathfrak{e}_{r}^{\bullet}\right\}$}

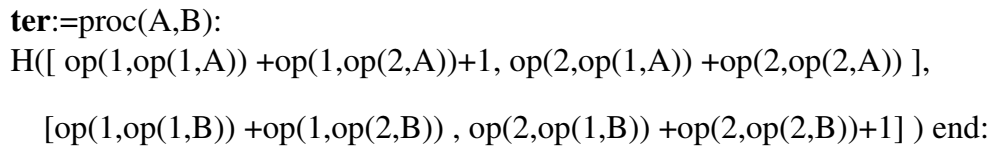

Ter:=proc $(X, Y)$ : seq( $\operatorname{seq}(\operatorname{ter}(\mathrm{op}(\mathrm{x}, \mathrm{X}), \mathrm{op}(\mathrm{y}, \mathrm{Y}))$,

$\mathrm{X}=1 . . \operatorname{nops}(\mathrm{X})), \mathrm{y}=1 . . \operatorname{nops}(\mathrm{Y}))$ end:

reslant $0:=[\mathrm{H}([0,-1 / 2],[0,-1 / 2])]:$

leslant $0:=[H([-1 / 2,0],[-1 / 2,0])]$ :

urslant: $=\operatorname{proc}(r)$ : if $r=1$ then $[\mathrm{H}([0,0],[0,0])]$ elif $r>1$ then

[ $\operatorname{Ter}($ urslant $(\mathrm{r}-1)$, reslant0), seq(Ter(urslant(r-1-k), urslant $(\mathrm{k})), \mathrm{k}=1 . . \mathrm{r}-2)$, 
$\operatorname{Ter}($ leslant0,urslant(r-1))] fi end:

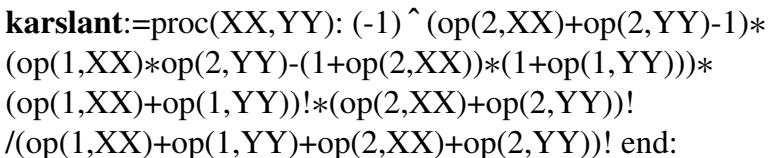

seslant $:=\mathrm{r} \rightarrow \operatorname{subs}(\mathrm{H}=$ karslant,urslant $(\mathrm{r})) ; \%$; (double click $)$

\subsubsection{Computation of the Stack-Coefficients for $\left\{\mathfrak{k}_{2 r}^{\bullet}\right\}$}

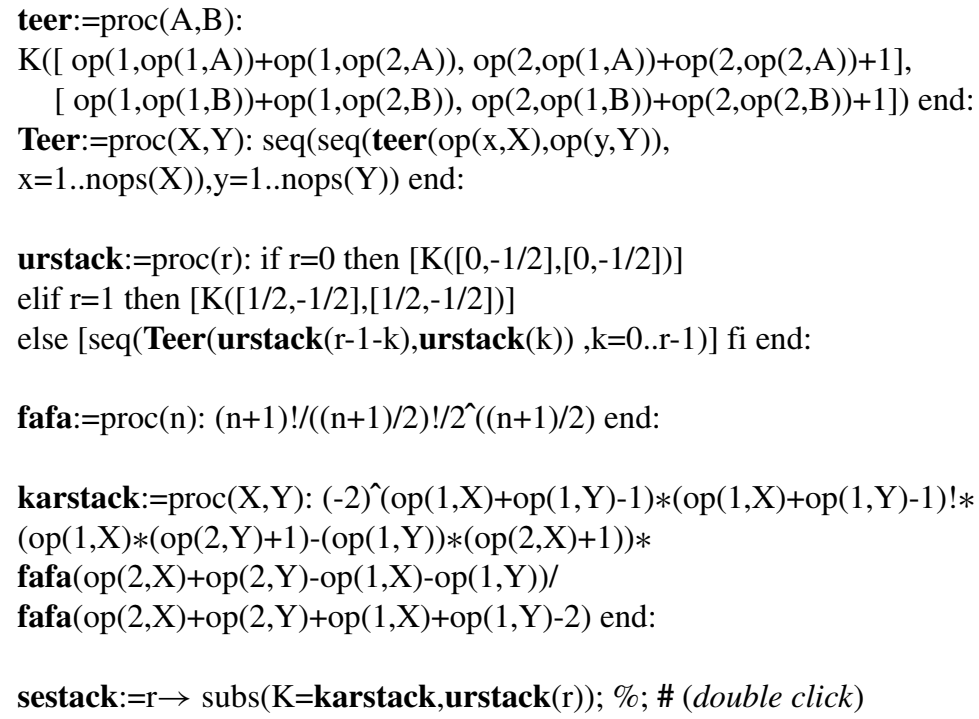

\subsubsection{Computation of the Alternals Series $\left\{\mathfrak{h} \mathfrak{e}_{r}^{\bullet}\right\}$ and $\left\{\mathfrak{k} \mathfrak{e}_{2 r}^{\bullet}\right\}$}

with(linalg);

multiply(seketel(r),seslant(r)); \# (gives $\mathfrak{h} \mathfrak{e}_{r}^{w}$ in the standard basis )

multiply(seketel(2r), seslack(2r)); \# (gives $\mathfrak{k e}_{2 r}^{w}$ in the standard basis )

\# Comment: To compute $h a_{r}^{\bullet}, k a_{2 r}^{\bullet}$, resp. $h i_{r}^{\bullet}, k i_{2 r}^{\bullet}$, use the above commands but with sekatal(r), resp. sekitil(r), in place of seketel(r).

\subsubsection{About the Moulds Mip $^{\bullet}$, Nip $^{\bullet}$, Rip $^{\bullet}$}

\# Comment: The following paragraphs permit the speedy computation of the moulds Mip*, $\mathrm{Nip}^{\bullet}$ (symmetrel) and Rip (symmetral) necessary for expanding any given eupolar bimould $S^{\bullet} \in \mathrm{Flex}(\mathrm{Pi})$ in the three bases (294) in function of the coefficients $\alpha_{r}$ (here noted a[r] for convenience). By suitably specializing a $[\mathrm{r}]$, the formulae yield the expansions of the basic bimoulds pil ${ }^{\bullet}$, ripil $^{\bullet}$, pil ${ }_{\mathrm{ev}}^{\bullet}$, ripil $_{\mathrm{ev}}^{\bullet}$ in all three bases (294). Our moulds Mip ${ }^{\bullet}$, Nip ${ }^{\bullet}$, Rip $^{\bullet}$ are indexed by positive integer sequences $\boldsymbol{n}$, which will be denoted here by $X=\left[n_{1}, \ldots, n_{r}\right]$. Each mould is dealt with in a separate paragraph, but the two following commands are required in each case: 
deb: $=X \rightarrow[\operatorname{seq}(o p(k, X), k=1 . . n o p s(X)-1)]:$

su: $=X \rightarrow \operatorname{add}(o p(k, X), k=1 . . n o p s(X))$ :

\subsubsection{Computation of the Symmetrel Mould Mip $\bullet$}

Mi $:=\operatorname{proc}(X): \operatorname{proc}(p)$ : if $\mathrm{p}=0$ or $\mathrm{p}>\mathrm{op}(+1, \mathrm{X})$ then 0

else $(-1)^{\wedge}(1+\operatorname{nops}(\mathrm{X})) * \mathrm{op}(-1, \mathrm{X}) * \mathbf{a}[\mathrm{su}(\mathrm{X})-\mathrm{p}]$ fi end: end:

Mij := proc $(\mathrm{X}):(-1)^{\wedge}(1+\operatorname{nops}(\mathrm{X})) * \mathrm{op}(-1, \mathrm{X}) * \mathbf{a}[\operatorname{su}(\mathrm{X})]$ end:

$\operatorname{Mip}:=\operatorname{proc}(\mathrm{X})$ option remember;

if $X=[]$ then 1

elif $\operatorname{nops}(\mathrm{X})=1$ and $\mathrm{op}(\mathrm{X})=1$ then $+\mathbf{a}[1]$

elif nops $(X)=1$ and $o p(X)>1$

then $+1 / \operatorname{su}(\mathrm{X}) * \mathbf{M i j}(\mathrm{X})+1 / \operatorname{su}(\mathrm{X}) * \operatorname{add}(\operatorname{Mip}([\mathrm{p}]) * \mathbf{M i}(\mathrm{X})(\mathrm{p})$,

$\mathrm{p}=1$..op $(\mathrm{X})-1)$

elif nops $(X)>1$ then

$+1 / \operatorname{su}(X) * \operatorname{add}(\mathbf{M i p}([\operatorname{seq}(\mathrm{op}(\mathrm{k}, \mathrm{X}), \mathrm{k}=1 . . \mathrm{i}-1)]) * \mathbf{M i j}([\operatorname{seq}(\mathrm{op}(\mathrm{k}, \mathrm{X})$,

$\mathrm{k}=\mathrm{i} . . \operatorname{nops}(\mathrm{X}))]), \mathrm{i}=1 . . \operatorname{nops}(\mathrm{X}))$

$+1 / \mathrm{su}(\mathrm{X}) * \operatorname{add}(\operatorname{add}(\operatorname{add}($

$\operatorname{Mip}([\operatorname{seq}(o p(k, X), k=1 . . i-1), p, \operatorname{seq}(o p(k, X), k=j+1 . . n o p s(X))]) *$

$\operatorname{Mi}([\operatorname{seq}(o p(k, X), k=i . . j)])(p)$,

$\mathrm{p}=1 . . \min (\operatorname{op}(\mathrm{i}, \mathrm{X}), \operatorname{add}(\mathrm{op}(\mathrm{k}, \mathrm{X}), \mathrm{k}=\mathrm{i} . . \mathrm{j})-1)), \mathrm{i}=1 . . \mathrm{j}), \mathrm{j}=1 . . \operatorname{nops}(\mathrm{X}))$

fi end:

\subsubsection{Computation of the Symmetrel Mould Nip ${ }^{\bullet}$}

$\mathbf{N i}:=\operatorname{proc}(X): \operatorname{proc}(\mathrm{p})$ : if $\mathrm{p}=0$ or $\mathrm{p}>\mathrm{op}(-1, \mathrm{X})$ then 0

else $(-1)^{\wedge}(1+\operatorname{nops}(\mathrm{X})+\mathrm{su}(\mathrm{X})-\mathrm{p}) * \mathrm{op}(+1, \mathrm{X}) * \mathbf{a}[\mathrm{su}(\mathrm{X})-\mathrm{p}]$ fi end: end:

$\mathbf{N i j}:=\operatorname{proc}(\mathrm{X}):(-1)^{\wedge}(\operatorname{nops}(\mathrm{X})+\operatorname{su}(\mathrm{X})) * \mathrm{op}(+1, \mathrm{X}) * \mathbf{a}[\operatorname{su}(\mathrm{X})]$ end:

Nip := proc $(X)$ option remember;

if $X=[]$ then 1

elif nops $(X)=1$ and $o p(X)=1$ then $+\mathbf{a}[1]$

elif $\operatorname{nops}(\mathrm{X})=1$ and $\mathrm{op}(\mathrm{X})>1$ then

$+1 / \mathrm{su}(\mathrm{X}) * \mathbf{N i j}(\mathrm{X})+1 / \mathrm{su}(\mathrm{X}) * \operatorname{add}(\mathbf{N i p}([\mathrm{p}]) * \mathbf{N i}(\mathrm{X})(\mathrm{p}), \mathrm{p}=1 . . \mathrm{op}(\mathrm{X})-1)$

elif nops $(\mathrm{X})>1$ then

$+1 / \operatorname{su}(X) * \operatorname{add}(\mathbf{N i p}([\operatorname{seq}(o p(k, X), \mathrm{k}=1 . . \mathrm{i}-1)]) * \mathbf{N i j}([\operatorname{seq}(\mathrm{op}(\mathrm{k}, \mathrm{X}), \mathrm{k}=\mathrm{i} . . \operatorname{nops}(\mathrm{X}))])$,

$\mathrm{i}=1$..nops $(\mathrm{X}))$

$+1 / \mathrm{su}(\mathrm{X}) * \operatorname{add}(\operatorname{add}(\operatorname{add}($

$\operatorname{Nip}([\operatorname{seq}(o p(k, X), k=1 . . i-1), p, \operatorname{seq}(o p(k, X), k=j+1 . . n o p s(X))]) *$

$\mathbf{N i}([\operatorname{seq}(o p(k, X), k=i . . j)])(p)$,

$\mathrm{p}=1 . . \min (\mathrm{op}(\mathrm{j}, \mathrm{X}), \operatorname{add}(\mathrm{op}(\mathrm{k}, \mathrm{X}), \mathrm{k}=\mathrm{i} . . \mathrm{j})-1)), \mathrm{i}=1 . . \mathrm{j}), \mathrm{j}=1 . . \operatorname{nops}(\mathrm{X}))$

fi end:

\subsubsection{Computation of the Symmetral Mould Rip $\bullet$}

Ri : $=\operatorname{proc}(X): \operatorname{proc}(p, q)$ : if $p+q<>\operatorname{add}(o p(k, X), k=1 . . \operatorname{nops}(X))$ then 0 elif nops $(X)=1$ and $p<o p(1, X)$ then $p * a[q]$ 


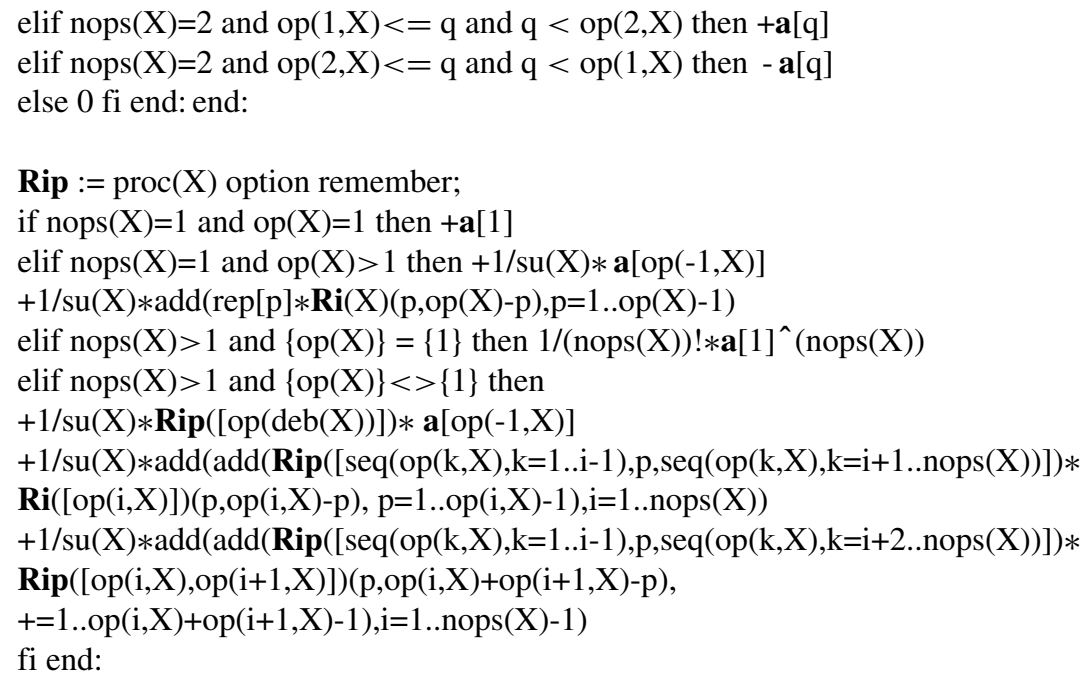

A toolkit for handling bisymmetrals and all flexion operations will soon be posted on our Webpage.

\subsection{Guide to the Annexed Tables}

About two dozen illustrative tables have been posted on our Webpage, ${ }^{104}$ in pdf format both for direct inspection and for easy copy-pasting. Each file begins with a Maple program capable of generating the file's contents (and much beyond) and then displays the results (usually up to length or $r=8$ or 10 or sometimes 12) either for their illustrative value or to make them available to non-Maple users.

\subsubsection{General Tools}

The files $\boldsymbol{a}_{\mathbf{1}}, \boldsymbol{a}_{\mathbf{2}}, \boldsymbol{a}_{\mathbf{3}}$ give the standard bases of the monogenous algebras Flex $(\mathrm{Pa}), \mathrm{Flex}(\mathrm{Pi})$, Flex $(\mathfrak{E})$. The files $\boldsymbol{a}_{4}, \boldsymbol{a}_{5}$ give the coefficients ("slant" and "stack") of the alternal series $\left\{\mathfrak{h} \mathfrak{e}_{r}^{\bullet}\right\},\left\{\mathfrak{k} \mathfrak{e}_{2 r}^{\bullet}\right\}$ in the standard basis.

\subsubsection{Recovering a General Bimould from Its gari-Dilators}

Symmetral bimoulds $S^{\bullet}$ whose gari-dilators $d i S^{\bullet}$ are in the "mock-differential algebra," i.e., of the form $\mathrm{di}^{\bullet}=\sum \alpha_{r} \mathfrak{r} \mathfrak{e}_{r}^{\bullet}$, themselves belong to a subalgebra Flex ${ }_{i n}(\mathfrak{E})$ much smaller than Flex $(\mathfrak{E})$ and can be expanded along three remarkable bases, smaller and more tractable than the standard basis:

$$
\begin{aligned}
\mathfrak{m} \mathfrak{e}_{n_{1}, \ldots, n_{s}}^{\bullet} & :=\operatorname{mu}\left(\mathfrak{m} \mathfrak{e}_{n_{1}}^{\bullet}, \ldots, \mathfrak{m} \mathfrak{e}_{n_{s}}^{\bullet}\right), \\
\mathfrak{n} \mathfrak{e}_{n_{1}, \ldots, n_{s}}^{\bullet} & :=\operatorname{mu}\left(\mathfrak{n} \mathfrak{e}_{n_{1}}^{\bullet}, \ldots, \mathfrak{n} \mathfrak{e}_{n_{s}}^{\bullet}\right), \\
\mathfrak{r} \mathfrak{e}_{n_{1}, \ldots, n_{s}}^{\bullet} & :=\operatorname{mu}\left(\mathfrak{r} \mathfrak{e}_{n_{1}}^{\bullet}, \ldots, \mathfrak{r} \mathfrak{e}_{n_{s}}^{\bullet}\right) .
\end{aligned}
$$

\footnotetext{
${ }^{104}$ At $<$ http://www.math.u-psud.fr/ ecalle/publi.html $>$ and <http://www.math.u-psud.fr/ ecalle/flexion.html>
} 
See (294) in Section 5. Each basis has its own advantages, and the files $\boldsymbol{b}_{\mathbf{1}}, \boldsymbol{b}_{\mathbf{2}}, \boldsymbol{b}_{\mathbf{3}}$ show how to expand $S^{\bullet}$ in each of them, using only two ingredients: the coefficients $\alpha_{r}$ of $\operatorname{dil}^{\bullet}$ and the three universal moulds $\operatorname{Mip}^{\bullet}$, Nip $^{\bullet}$, Rip $^{\bullet}$.

\subsubsection{Recovering pil $^{\bullet}$, ripil ${ }^{\bullet}$ from Their Gari-Dilators}

The files $\boldsymbol{c}_{\mathbf{1}}, \boldsymbol{c}_{\mathbf{2}}, \boldsymbol{c}_{\mathbf{3}}$ and $\boldsymbol{c}_{\mathbf{4}}, \boldsymbol{c}_{\mathbf{5}}, \boldsymbol{c}_{\mathbf{6}}$ apply the above universal expansions to the standard bisymmetral pil $^{\bullet}$ and its gari-inverse ripil $^{\bullet}$. The corresponding specializations of Mip $^{\bullet}$, Nip $^{\bullet}$, Rip $^{\bullet}$ (integer-indexed and rational-valued) possess interesting, Bernoulli-like arithmetical properties.

\subsubsection{Recovering pil $_{\text {ev }}^{\bullet}$, ripil $\bullet_{\text {ev }}^{\bullet}$ from Their gari-Dilators}

The files $\boldsymbol{d}_{\mathbf{1}}, \boldsymbol{d}_{\mathbf{2}}, \boldsymbol{d}_{\mathbf{3}}$ and $\boldsymbol{d}_{\mathbf{4}}, \boldsymbol{d}_{\mathbf{5}}, \boldsymbol{d}_{\mathbf{6}}$ similarly expand the even factors pil $_{e v}^{\bullet}$, ripil $l_{e v}^{\bullet}$, leading to more economical expansions of our bisymmetrals, while isolating their essential, even part.

\subsubsection{Recovering pal $^{\bullet}$, pal $_{e v}^{\bullet}$, pal $_{\text {evv }}^{\bullet}$ from Their mu-Dilators}

This is the object of file $\boldsymbol{e}_{1}$, to be completed by other tables about the mould $\mathrm{Han}^{\bullet}$ occurring in the expansion 303.

\subsubsection{Regular Bisymmetrals and Associated Bialternals}

The file $\boldsymbol{f}_{\mathbf{1}}$ deals with the regular bialternals $l a r^{\bullet}$ and $r a l^{\bullet}$ (which by gari-postcomposition link pal ${ }^{\bullet}$ and par $^{\bullet}$ to one another: see Section 9) and gives their expansions along the standard basis (since for them no simpler basis is available). The file $\boldsymbol{f}_{\mathbf{2}}$ provides similar expansions for the dilators dilar ${ }^{\bullet}$ and diral (bialternals of the "first kind": see Section 9) and file $\boldsymbol{f}_{\mathbf{3}}$ does the same for the singulator-related bimoulds visla ${ }^{\bullet}$ and visra ${ }^{\bullet}$ (bialternals of the "second kind": see Section 10).

\subsubsection{Construction of tal ${ }^{\bullet}$ and Its Even/Odd Factors}

The file $g_{1}$ deals with the factorizations

$$
\operatorname{tal}^{\bullet}=\operatorname{gari}\left(\operatorname{tal}_{\mathrm{od}}, \operatorname{tal}_{\mathrm{ev}}\right) \quad \text { and } \operatorname{tal}_{\mathrm{ev}}^{\bullet}=\operatorname{mu}\left(\operatorname{tal}_{\mathrm{lev}}, \operatorname{tal}_{\mathrm{rev}}\right)
$$

and the file $\boldsymbol{g}_{2}$ deals with the factorizations

$$
\mathrm{tal}^{\bullet}=\mathrm{mu}\left(\mathrm{tal}_{\mathrm{evv}}, \mathrm{tal}_{\mathrm{odd}}\right) \quad \text { and } \mathrm{tal}_{\mathrm{evv}}^{\bullet}=\mathrm{mu}\left(\mathrm{tal}_{\mathrm{levv}}, \mathrm{tal}_{\mathrm{revv}}\right) .
$$

The non-trivial factors are given via their dilators, which in turn are defined through their coefficients in one of the two natural bases of $L U(Q a, c I)$, namely the one that is spanned by the alternals $Q a_{n_{1}, \ldots, n_{s}}^{\bullet}$ so defined:

$$
\mathrm{Qa}_{n_{1}, \ldots, n_{s}}^{\bullet}:=\operatorname{lu}\left(\mathrm{Qa}_{n_{1}}^{\bullet}, \ldots, \mathrm{Qa}_{n_{s}}^{\bullet}\right) \quad \text { with } \mathrm{Qa}_{n}^{\bullet}:=\operatorname{lu}(c \mathrm{I}^{\bullet}, \overbrace{\mathrm{Qa}^{\bullet}, \ldots, \mathrm{Qa}^{\bullet}}^{(n-1) \text { times }}) .
$$

The other natural basis of $L U(Q a, c I)$ is spanned by the alternals $\mathrm{Ka}_{n_{1}, \ldots, n_{s}}^{\bullet}$

$$
\mathrm{Ka}_{n_{1}, \ldots, n_{s}}^{\bullet}:=\operatorname{lu}\left(\mathrm{Ka}_{n_{1}}^{\bullet}, \ldots, \mathrm{Ka}_{n_{s}}^{\bullet}\right) \quad \text { with } \quad \mathrm{Ka}_{n}^{\bullet}:=\operatorname{lu}(\mathrm{Qa}^{\bullet}, \overbrace{c \mathrm{I}^{\bullet}, \ldots, c \mathrm{I}^{\bullet}}^{(n-1) \text { times }}) .
$$


When comparing the expansions of our trigonometric dilators in these two bases, curiousthough limited and still poorly understood—duality phenomena become noticeable.

Open Access This article is distributed under the terms of the Creative Commons Attribution 4.0 International License (http://creativecommons.org/licenses/by/4.0/), which permits unrestricted use, distribution, and reproduction in any medium, provided you give appropriate credit to the original author(s) and the source, provide a link to the Creative Commons license, and indicate if changes were made.

\section{References}

1. Chapoton, F.: On some anticyclic operads. Algebr. Geom. Topol. 5, 53-69 (2005)

2. Ecalle, J.: ARI/GARI, la dimorphie et l'arithmétique des multizêtas: un premier bilan. J. Théor. Nombres Bordx. 15, 411-478 (2003)

3. Ecalle, J.: Multizetas, perinomal numbers, arithmetical dimorphy. Ann. Fac. Toulouse 4, 683-708 (2004)

4. Ecalle, J.: The flexion structure and dimorphy: flexion units, singulators, generators, and the enumeration of multizeta irreducibles. Ann. Scuo. Norm. Pisa 2, 27-211 (2011). Asymptotics in Dynamics, Geometry and PDEs; Generalized Borel Summation; ed. Costin, O., Fauvet, F., Menous, F., Sauzin, D

5. Ecalle, J.: The eutrigonometric flexion algebra and the bimould $t_{a l} l^{\bullet} / t^{\bullet}$. (forthcoming)

6. Ecalle, J.: Maximal finitary flexion algebras. (forthcoming)

7. Plouffe, S., Sloane, N.J.A.: The Encyclopedia of Integer Sequences. Academic Press (1995) 